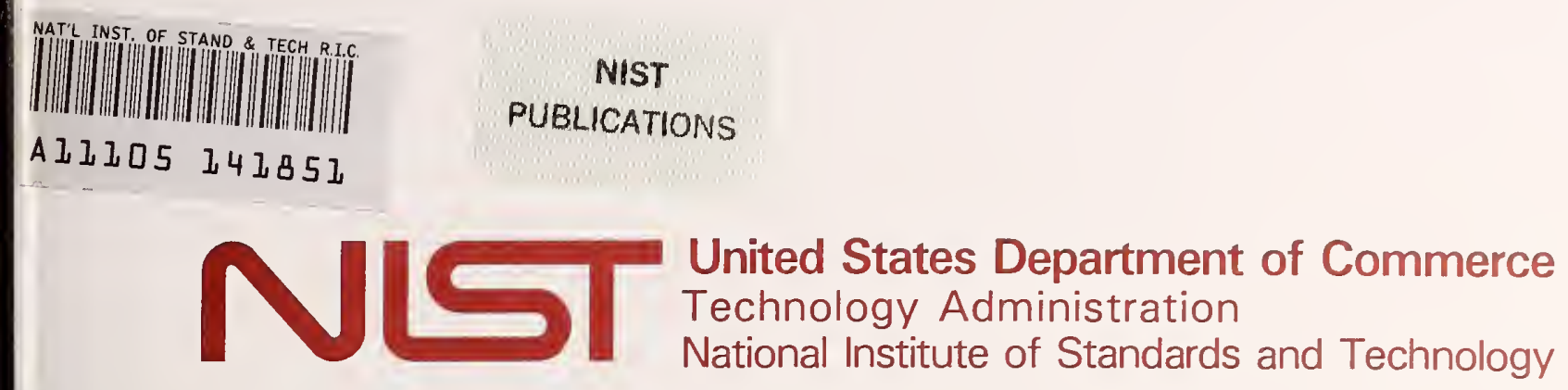

NIST Technical Note 1387

\title{
Evaluation and Correlation of Steam Solubility Data for Salts and Minerals of Interest in the Power Industry
}

\author{
Allan H. Harvey \\ James C. Bellows
}

xc

100

15753

vo.1387

1997 

NIST Technical Note 1387

\title{
Evaluation and Correlation of Steam Solubility Data for Salts and Minerals of Interest in the Power Industry
}

\author{
Allan H. Harvey \\ James C. Bellows*
}

Physical and Chemical Properties Division

Chemical Science and Technology Laboratory

National Institute of Standards and Technology

325 Broadway

Boulder, Colorado 80303

* Steam Turbine Engineering

Westinghouse Power Generation

4400 Alafaya Trail

Orlando, Florida 32826

March 1997

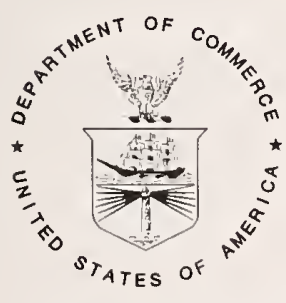

U.S. DEPARTMENT OF COMMERCE, William M. Daley, Secretary TECHNOLOGY ADMINISTRATION, Mary L. Good, Under Secretary for Technology NATIONAL INSTITUTE OF STANDARDS AND TECHNOLOGY, Arati Prabhakar, Director 
National Institute of Standards and Technology Technical Note Natl. Inst. Stand. Technol., Tech. Note 1387, 88 pages (March 1997) CODEN:NTNOEF

\section{U.S. GOVERNMENT PRINTING OFFICE \\ WASHINGTON: 1997}

For sale by the Superintendent of Documents, U.S. Government Printing Office, Washington, DC 20402-9325 


\section{CONTENTS}

1. Introduction $\ldots \ldots \ldots \ldots \ldots \ldots \ldots \ldots \ldots \ldots \ldots \ldots \ldots \ldots \ldots$

2. Boundaries of Study .......................... 2

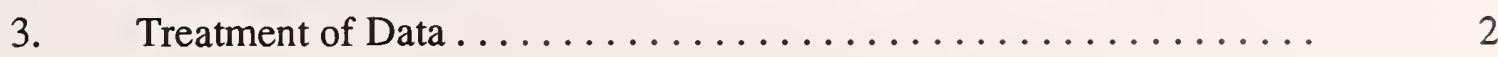

4. Correlating Data $\ldots \ldots \ldots \ldots \ldots \ldots \ldots \ldots \ldots \ldots \ldots \ldots \ldots \ldots \ldots$

5. Sodium Chloride ............................. 4

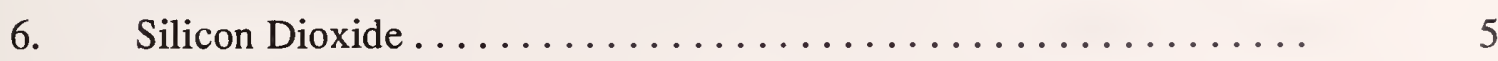

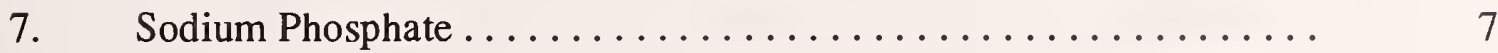

8. Sodium Sulfate $\ldots \ldots \ldots \ldots \ldots \ldots \ldots \ldots \ldots \ldots \ldots \ldots \ldots \ldots \ldots$

9. Copper Oxides .......................... 10

10. Sodium Hydroxide . . . . . . . . . . . . . . . . . . . . 12

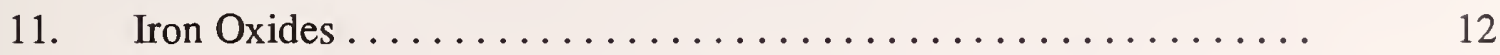

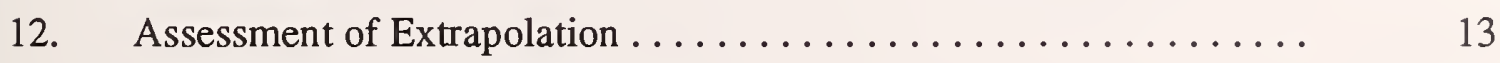

13. Summary of Solubility Calculations $\ldots \ldots \ldots \ldots \ldots \ldots \ldots \ldots \ldots$

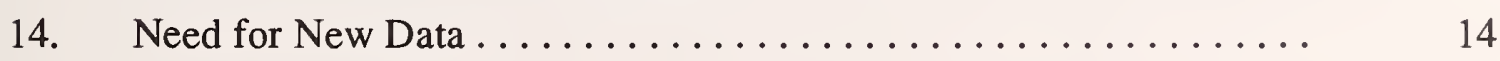

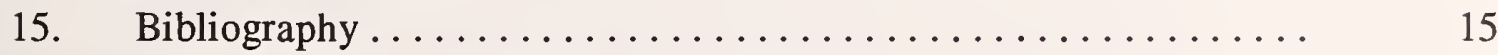

Appendix A. Solubility Data ....................... A-1

Cupric Oxide $(\mathrm{CuO}) \ldots \ldots \ldots \ldots \ldots \ldots \ldots \ldots \ldots \ldots \ldots \ldots \ldots \ldots \ldots \ldots \ldots \ldots \ldots$

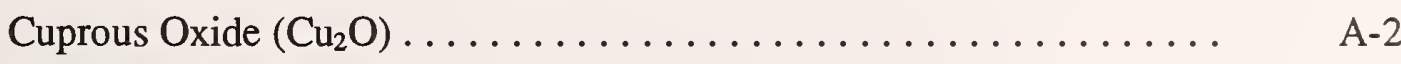

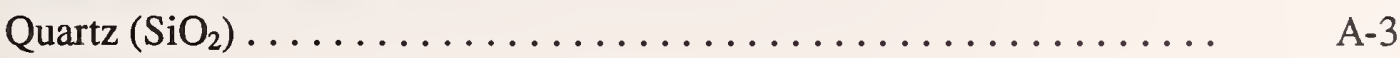

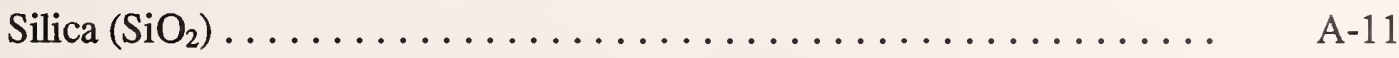

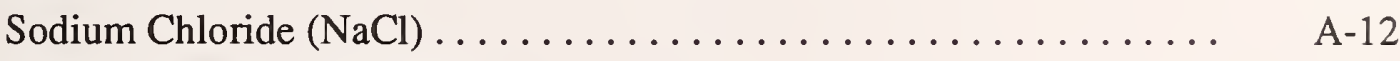

Sodium Hydroxide $(\mathrm{NaOH}) \ldots \ldots \ldots \ldots \ldots \ldots \ldots \ldots \ldots \ldots \ldots \ldots \ldots$

Sodium Phosphate $\left(\mathrm{Na}_{3} \mathrm{PO}_{4}\right) \ldots \ldots \ldots \ldots \ldots \ldots \ldots \ldots \ldots \ldots \ldots \ldots \ldots \ldots \ldots \ldots$

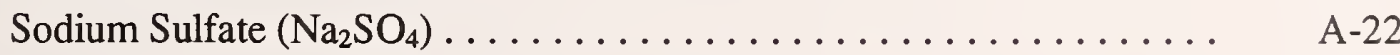

Appendix B. Vapor Pressures ......................... B-1

Appendix C. Vapor-Liquid Partitioning for $\mathrm{Na}_{3} \mathrm{PO}_{4}$ and $\mathrm{Na}_{2} \mathrm{SO}_{4} \ldots \ldots \ldots$ C-1

Appendix D. Examination of Extrapolation Behavior ............ D-1

Appendix E. Sample Solubility Calculations ................ E-1 


\section{List of Symbols}
A, B, D coefficients in solubility equations
$\mathrm{K}$ equilibrium constant for hydration reaction
$\mathrm{P} \quad$ pressure, $\mathrm{MPa}$
S solute species
$\mathrm{T}$ absolute temperature, $\mathrm{K}$
a activity of a species
$\mathrm{m}$ number of waters in hydration reaction
$\mathrm{m}_{1}, \mathrm{~m}_{2}, \mathrm{~m}_{3}$ coefficients in solubility equations
S solid phase
V vapor phase
$\mathrm{X}_{\mathrm{S}} \quad$ solute mole fraction

\section{Greek Letters}

$\rho$

water/steam density, $\mathrm{mol} / \mathrm{L}$ 


\title{
Evaluation and Correlation of Steam Solubility Data for Salts and Minerals of Interest in the Power Industry
}

\author{
Allan H. Harvey and James C. Bellows* \\ Physical and Chemical Properties Division \\ Chemical Science and Technology Laboratory \\ National Institute of Standards and Technology \\ 325 Broadway \\ Boulder, CO 80303
}

\begin{abstract}
We performed a literature search for data on the solubility in steam of several salts and minerals of interest in the steam power industry. The specific solutes of interest were sodium chloride, sodium hydroxide, sodium sulfate, sodium phosphate, iron oxides, copper oxides, and silicon dioxide. The region of primary interest was temperatures from $300{ }^{\circ} \mathrm{C}$ to $640{ }^{\circ} \mathrm{C}$ and pressures from $2 \mathrm{MPa}$ to 4 $\mathrm{MPa}$. Some effort was made to choose among discrepant data sets and to assess the level of uncertainty in the solubility determinations. Simple functional forms, expressing the solubility as a function of the temperature and density of pure steam at the experimental conditions, were fit to the data. For solutes where there are no data in the region of interest, various extrapolation methods were used and estimates were made of the uncertainty involved in the extrapolation. A discussion is given of the most critical data needs in this area.
\end{abstract}

Key words: aqueous solutions; combustion turbine; deposition; mineral; power generation; salt; solid-fluid equilibria; solubility; steam; turbine

\section{Introduction}

Deposition of solids from expanding steam is a significant concern in the power industry. Deposition in steam turbines is well known. Depending on the deposited material and the location in the turbine, deposits can cause corrosion or constriction of passages. Constriction of passages causes higher pressure drops, reduced efficiency, and reduced capacity. Corrosion can lead to failures and expensive repairs. Investigations into steam purity and the solubility of salts in steam have been in progress for over 50 years [1].

The problem of deposition has developed new urgency as steam cooling is proposed for advanced combustion turbines. In these turbines, some of the passages will be substantially smaller than in the steam turbines. A small amount of deposition can block a passage. When the passage is blocked, the region will overheat and the part will fail. Additionally, the steam will be in contact with substantially hotter metal. Hot corrosion is unknown in steam turbines, but is well

*Steam Turbine Engineering, Westinghouse Power Generation, 4400 Alafaya Trail, Orlando, Florida 32826 
known in combustion turbines due to higher temperatures. Sodium sulfate is well known as a cause of hot corrosion, where in steam turbines it is only a mild corrodent. Deposition of materials that are benign in steam turbines can cause corrosion in the combustion turbine.

This project was undertaken to collect and, if possible, correlate all available data for the solubility in steam of the following solutes: sodium chloride, sodium hydroxide, sodium sulfate, sodium phosphate, iron oxides, copper oxides, and silicon dioxide. The correlations will be useful in developing steam purity recommendations for the steam conditions encountered in the advanced turbines.

\section{Boundaries of Study}

The temperature range considered was $200{ }^{\circ} \mathrm{C}$ to $900{ }^{\circ} \mathrm{C}$ (with $300{ }^{\circ} \mathrm{C}$ to $640{ }^{\circ} \mathrm{C}$ of primary interest) and the pressure range considered was 1 to $6 \mathrm{MPa}$ (with 2 to $4 \mathrm{MPa}$ of primary interest). These conditions correspond to very low steam densities (on the order of 0.5 to 1 $\mathrm{mol} / \mathrm{L}$ ) at which few reliable data exist. It was therefore necessary to collect and include in the correlations data at higher pressures (and therefore higher densities). Some data sufficiently far from the region of interest were not of any use in the study; the prime example is the abundant data for solubilities of some of these substances in liquid water. While some data in this category were cataloged if they were encountered in the literature search, no attempt was made to be comprehensive except in and near the region of interest.

The work proceeded on the assumption that the solutes remained chemically intact, undergoing no hydrolysis, decomposition, or other chemical reaction. This is not true for some solutes; for example, Galobardes et al. [2] found that hydrolysis was not insignificant at high temperatures in their measurements of $\mathrm{NaCl}$ solubility. Sodium sulfate is also known to hydrolyze to some extent and to undergo partial decomposition at high temperatures. Such reactions were ignored on the grounds that considering them would have made the problem intractable and that the reaction products would likely be at least as soluble as the intact solutes, meaning that the assumptions of no hydrolysis or dissociation would probably provide a conservative estimate for solids deposition calculations. For similar reasons, the possible enhancement of the solubility of one solute by the presence of another (for example, the effect of dissolved ammonia on solutes whose solubility is sensitive to $\mathrm{pH}$ ) was not considered.

Also, the work was limited to the equilibrium solubility. This meant that transport of solutes by mechanical carryover was not considered. It also meant that there was no consideration of kinetic aspects of solute deposition. It is possible for situations to arise where the equilibrium favors precipitation, but the kinetics are such that precipitation will not happen in the time available. Such considerations are beyond the scope of this study, but assuming precipitation when thermodynamically possible is again a conservative assumption in this context.

\section{Treatment of Data}

All data were entered into a spreadsheet in the units in which they were originally reported. They were then converted to units of temperature in kelvins, pressure in megapascals, 
the density of pure water at that temperature and pressure in moles per liter, and the solubility in mole fraction. In most cases, the temperature and pressure were reported and the density of water was calculated from the 1995 IAPWS formulation for the properties of water and steam, hereafter referred to as the Steam Tables [3]. Some data were reported at the vapor-liquid saturation boundary with just temperature or pressure given, in which case the other saturation condition and the corresponding density were calculated from the Steam Tables. In a few cases, the temperature and density were reported and the pressure of pure water was calculated from the Steam Tables. The data tabulations in Appendix A indicate which variables were measured for each data set and the units in which the data were originally reported. It should be noted that these procedures will not exactly match the densities at the experimental conditions, because the PVT behavior of the solid-saturated mixture will not be identical to that of pure water. This mismatch should be small at conditions where the solubility is small (which is for the most part the case in this work), but could be significant at conditions where the solubility is larger.

\section{Correlating Data}

There are two fundamental ways to describe the solubility of a solid in a gas or supercritical fluid. The first is a physical model, where the volatility (as represented by the fugacity) of the solute is given to a first approximation by its vapor pressure and is then augmented by a Poynting correction (which gives the effect on the fugacity of a condensed substance due to pressure above its saturation pressure) and a fugacity coefficient to account for nonideality in the vapor phase. Fugacity coefficients can be calculated with a variety of models. However, the substances of interest in this work show solubilities in steam many orders of magnitude larger than would be expected based on their vapor pressures. This suggests that the effects governing the solubility are too strong for a physical, fugacity-coefficient-based model. The available vapor-pressure data for each solute are summarized in Appendix B.

The second option is a reaction model, where the solubility is viewed as a complexation between some number of solvent molecules and a solute molecule. This has been the customary approach for describing the solubility of these compounds, and it is the one adopted in this work. If we consider the solute to be solvated by $\mathrm{m}$ solvent molecules, the reaction is written

$$
\mathrm{S}(\mathrm{s})+\mathrm{mH}_{2} \mathrm{O}(\mathrm{v}) \Leftrightarrow \mathrm{S} \cdot \mathrm{mH}_{2} \mathrm{O}(\mathrm{v})
$$

where $\mathrm{S}$ is the solute. Assuming the activity of the solid to be 1 (which ignores a Poynting correction, but that will be negligible at low and moderate pressures, especially at the relatively high temperatures of interest here), the thermodynamic equilibrium constant $K(T)$ for that reaction can be written as

$$
\mathrm{K}(\mathrm{T})=\frac{\mathrm{a}\left(\mathrm{S} \cdot \mathrm{mH}_{2} \mathrm{O}\right)}{\left[\mathrm{a}\left(\mathrm{H}_{2} \mathrm{O}\right)\right]^{\mathrm{m}}},
$$

where a is the activity. At this point things become difficult. The activity of pure water is just its fugacity, but there is no way in general to predict or describe the activity of the hypothetical hydrated solute complex. We must therefore begin making reasonable approximations. The first 
is to replace the activity of the complex by some direct measure of how much solute is dissolved, such as its mole fraction or partial pressure. Second, it has been found that better correlations are obtained by replacing the activity of water with its density. This leads to equations of the form

$$
\ln \left(\mathrm{x}_{\mathrm{S}}\right)=\mathrm{f}(\mathrm{T})+\mathrm{m} \ln \rho_{\mathrm{H}_{2} \mathrm{O}} \quad(-\ln \mathrm{P}),
$$

where $\mathrm{x}_{\mathrm{s}}$ is the mole fraction of solute, $\rho_{\mathrm{H} 2 \mathrm{O}}$ is the density of the solvent water and the last $(-\ln \mathrm{P})$ term is only included if partial pressure (rather than mole fraction) is used to express the amount of solute. The function $\mathrm{f}(\mathrm{T})$ is no longer the thermodynamic equilibrium constant, but now also has to make up for other approximations in the model. The hydration number $m$ could in general be a function of density and/or temperature.

\section{Sodium Chloride}

$\mathrm{NaCl}$ has by far the most solubility measurements of any substance of interest in this work in the region of interest. Most of the measurements are in good agreement at densities above $2 \mathrm{~mol} / \mathrm{L}$; below that, the measurements diverge considerably. Data considered were restricted to steam densities below $10 \mathrm{~mol} / \mathrm{L}$. The data of Alekhin and Vakulenko [4] were excluded because their values were about two orders of magnitude above those of the vast majority of investigators. Others have observed [ 5 , for example] that their experimental setup might be prone to adsorption effects. The data of Sourirajan and Kennedy [6] were about one order of magnitude above those of other investigators and show a very different trend with density than what others observe; these data were also excluded. Finally, the early data of Spillner [1] were excluded; they fell somewhat above the general trend and exhibited a large amount of scatter. This resulted in 12 references [2, $5,7-16]$ being used in the fit.

At densities below $2 \mathrm{~mol} / \mathrm{L}$, there is a dichotomy in the data. Some data show a continuous steep decrease in solubility with decreasing density, while other (primarily Russian) data indicate that the solubility levels off or at least decreases less quickly. Shvedov [17] suggested that at least some of the latter data, because they were based on measurements of chloride concentration, are artificially high due to the production of $\mathrm{HCl}$ by hydrolysis. For this reason, and because it was a more conservative estimate in the context of potential precipitation from steam, we chose to fit the data in this region with the lower solubilities. We therefore excluded the low-density (again below $2 \mathrm{~mol} / \mathrm{L}$ ) data of Martynova and Samilov [12] and Styrikovich et al. [8], and also the low-temperature (at and below $402{ }^{\circ} \mathrm{C}$ ) low-density data of Sastry [11] and the low-temperature (below $400^{\circ} \mathrm{C}$ ) data of Bischoff et al. [15]. In addition, to avoid giving undue weight to the data from Sastry [11] in which over 200 points were reported, those points not excluded from the fit were given a weight of 0.2 relative to the other data. Similarly, the approximately 50 points along a single isobar by Aleinikov et al. [9] were each given a weight of 0.5 .

The data were fit to a simple equation of the form

$$
\ln \left(\mathrm{x}_{\mathrm{S}}\right)=\mathrm{A}+\mathrm{B} / \mathrm{T}+\left(\mathrm{m}_{1}+\mathrm{m}_{3} / \mathrm{T}\right) \ln \rho-\ln \mathrm{P},
$$


with values of $\mathrm{A}=-7.92886, \mathrm{~B}=-4852.47, \mathrm{~m}_{1}=4.49961$, and $\mathrm{m}_{3}=-0.0013$. In eq (4), the temperature must be in $\mathrm{K}$, the density in $\mathrm{mol} / \mathrm{L}$, and the pressure in $\mathrm{MPa}$.

Figure 1 shows the range of temperature and density covered by the $\mathrm{NaCl}$ solubility data (data sets that were discarded completely are not included in the figure). Figures 2 and 3 show the deviations of the experimental data from the predicted values of $\ln \left(\mathrm{x}_{\mathrm{s}}\right)$ as a function of density and of temperature for eq (4). Figures 4 through 7 show how the data are fit along four isotherms (where an isotherm is defined as encompassing a finite temperature range in order to include enough data to produce a meaningful graph). Figures 4 through 7 also show the points from the references not used in the fit $[1,4,6]$; it can be seen that especially [4] and [6] deviate badly from the general trend of the data. Figure 5 (the $450^{\circ} \mathrm{C}$ isotherm) also shows a few points from Baierlein [18], who did not report whether the phase coexisting with his salt-saturated vapor was liquid or solid. We have identified those points which (based on knowledge of the three-phase line) we believe to have been vapor-solid measurements. Those points are shown on figure 5 and listed in Appendix A. They were not used in the fit, but are in fair agreement with the data that were used.

There is substantial uncertainty in the solubility at low densities. While we chose to fit the points with lower solubilities partly based on the analysis of Shvedov [17] concerning hydrolysis, Galobardes et al. [2] claimed that hydrolysis was at most a moderate (on the order of 10-20 percent) effect at the conditions of their measurements. Since this issue is still not clearly resolved, and since several studies (at least one of which [11] measured sodium concentration which should not be affected by hydrolysis) report higher solubilities at these densities, we cannot rule out the possibility that the solubility of $\mathrm{NaCl}$ at low steam densities is significantly higher than that given by eq (4).

\section{Silicon Dioxide}

There is a great deal of measured solubility data for $\mathrm{SiO}_{2}$, but unfortunately the vast majority is for geological conditions at pressures far beyond our range of interest (data in this category encountered in our literature search are, however, still listed in the bibliography and with the other solubility data in Appendix A). In the region of interest, there is a large amount of data from Heitmann [19], a moderate amount of data on two isotherms from Wendlandt [20], a few points from Morey and Hesselgesser [21, 22], and one point at a relatively high density from Kennedy [23]. Figure 8 shows the range of temperature and density covered by the $\mathrm{SiO}_{2}$ solubility data; only points included in the fit are shown in the figure.

A question with silicon dioxide is the form of the solid to be considered. In the temperature range of interest, quartz is the thermodynamically stable solid form. However, it is also easy to obtain an amorphous silica phase. Free-energy considerations indicate that the quartz phase should have a smaller solubility, so that the conservative approach would be to consider and correlate quartz's solubility. It might be, however, that nonthermodynamic factors would inhibit the precipitation of quartz so that the deposited phase would be amorphous silica. According to the data of Heitmann [19], quartz has a much lower solubility in water at liquid-like densities, but the two solubilities are about equal (the amorphous silica solubility still appears to be somewhat higher, but perhaps not within the experimental uncertainty) in low-density steam. We therefore 
supplemented the quartz data with the portion of Heitmann's data for amorphous silica solubility taken in steam at relatively low pressures $\left(50 \mathrm{~kg} / \mathrm{cm}^{2}\right.$ and below). Each of these points was given 0.2 times the weight of the quartz points in the fit.

The two major data sources in the region of interest are Heitmann [19] and Wendlandt [20]. Unfortunately, these measurements disagree with each other significantly (though not as badly as the low-density $\mathrm{NaCl}$ data mentioned in the previous section). They are in fairly good agreement at higher densities, but at lower densities near $1 \mathrm{~mol} / \mathrm{L}$ the solubilities of Wendlandt are lower by about a factor of 3 to 5. Examination of the dissertations containing the data [20, 24] did not reveal any obvious flaws. Both workers used a flow method to take their data, and both report examining the dependence of solubility on flowrate to ensure that equilibrium was reached. That examination appears to have been more extensive in Heitmann's work [24]; Wendlandt [20] only mentions that this flowrate independence was determined in previous experiments (which may not have even been on quartz, since several solutes are examined in his work). One might think of using the few points of Morey and Hesselgesser [21] in the region to decide between the two data sets. Unfortunately, the data from [21] are in closer agreement with Wendlandt's data on the $400^{\circ} \mathrm{C}$ isotherm, but in closer agreement with Heitmann on the $500{ }^{\circ} \mathrm{C}$ isotherm. Ultimately, we decided to weight each of Wendlandt's points equally to each of Heitmann's quartz points. This had the effect of giving somewhat higher weight overall to Heitmann's data [19], since he reported more points.

Since our solubility estimates rely heavily on the data of Heitmann, we discuss them briefly here. Data for solubility of $\mathrm{SiO}_{2}$ in water and steam were published in two places [19, 25], with the data in [25] a subset of that in [19]. The original source was Heitmann's dissertation [24]. This contains points at a few more conditions than were reported in the publications; we do not know whether there was a reason for omitting these points in the publications. To be safe, we did not include these extra points in our fit. What is more disturbing is the way Heitmann discarded some measurements. The dissertation reports all measured points, but marks with a cross those that were discarded. These points amounted to about 20 percent of all the measured points. The reason given by Heitmann for discarding these points was that they did not fit well on the solubility diagram he constructed (figure 1 in [19] and figure 7 in [25]). The discarded points are sometimes quite close to points that were not discarded; at other times they differ in solubility by about a factor of 2. We conclude that the reproducibility of Heitmann's measurements is probably not better than approximately a factor of 2 . It is encouraging that his solubilities at higher densities where other data do exist are not too different from those measured by others.

We tested several forms; the data were ultimately fit to the equation

$$
\ln \left(x_{S}\right)=A+D T+\left(m_{1}+m_{2} \rho+m_{3} T\right) \ln \rho-\ln P
$$

with values of $\mathrm{A}=-21.6047, \mathrm{D}=0.0135587, \mathrm{~m}_{1}=1.05555, \mathrm{~m}_{2}=0.0941005$, and $\mathrm{m}_{3}$ $=0.000912888$. Figures 9 and 10 show the deviations of experimental values of $\ln \left(\mathrm{x}_{\mathrm{s}}\right)$ from eq (5) as a function of density and temperature. Figures 11 and 12 show comparisons of the experimental data and eq (5) for the $400{ }^{\circ} \mathrm{C}$ and $500^{\circ} \mathrm{C}$ isotherms. 


\section{Sodium Phosphate}

The situation for $\mathrm{Na}_{3} \mathrm{PO}_{4}$ is complicated by the chemistry. The $\mathrm{Na} / \mathrm{P}$ ratio in aqueous solution can vary over a wide range. The solids in equilibrium can have various compositions, and under some circumstances a second liquid phase can form. Some of this chemistry is summarized by Marshall [26] and by Wetton [27]. However, it appears that one can, at least approximately, focus on a $3: 1 \mathrm{Na} / \mathrm{P}$ ratio and ignore most of the chemistry, since the equilibrium solid from that ratio has a $\mathrm{Na} / \mathrm{P}$ ratio near 2.8 [27]. This allows us to make rough solubility estimates based on earlier data for $\mathrm{Na}_{3} \mathrm{PO}_{4}$ which did not take the extra chemistry into account. One would hope that the complex chemistry would be less important in the steam phase.

Unfortunately, we could find no data for $\mathrm{Na}_{3} \mathrm{PO}_{4}$ solubility in steam. We therefore used an indirect estimation method. First, the measured solubility in liquid water at saturation at a high temperature was combined with vapor-liquid partitioning data to estimate the solubility in the saturated vapor at that temperature. Then we extrapolated from that point by assuming that the dependence of the solubility on temperature and density was the same as for a model solute.

The highest temperature at which there are measurements for liquid-phase solubility is $350^{\circ} \mathrm{C}$. The mole-fraction solubility at that temperature is $1.65 \times 10^{-4}$ according to Schroeder et al. [28] and $5.97 \times 10^{-4}$ according to Wetton [27]. We decided to give more weight to the more recent study [27], since the complex chemistry was not appreciated at the time of the Schroeder et al. [28] work. We therefore used a value of $5.0 \times 10^{-4}$.

For the distribution between vapor and liquid, we used the data of Goodstine [29, supplemented by additional data courtesy of ABB Combustion Engineering], who measured the partitioning of sodium between vapor and liquid phases at various concentrations and pressures. The data we used were graphs of sodium concentration (in parts per million) in coexisting vapor and liquid phases. Values read from the graph, conversion of those data to mole fractions, and derived partition coefficients are given in tabular form in Appendix C, which also gives the results of similar calculations with $\mathrm{Na}_{2} \mathrm{SO}_{4}$. The pressures were translated to temperatures by using vapor pressures of pure water from the Steam tables [3], which should not introduce much error since the solute concentrations were low. Since none of Goodstine's measurements was at solid saturation, the partition coefficients (which were concentration-dependent) had to be extrapolated to saturation. In this extrapolation, we made the conservative assumption that the partition coefficient leveled off as saturation was approached. Based on the extrapolated values of the partition coefficient at saturation at various temperatures, we estimated a partition coefficient of $5.6 \times 10^{-4}$ at saturation at $350^{\circ} \mathrm{C}$, which gives a vapor mole fraction of $2.8 \times 10^{-7}$.

To get an equation for the solubility of sodium phosphate at any condition, we then assume that its change in solubility with density and temperature is the same as for some model substance. We choose sodium chloride as the model substance since it has the most data in the region of interest. We use the $\mathrm{NaCl}$ equation to extrapolate from the saturated vapor at $350{ }^{\circ} \mathrm{C}$, where the density of pure water is $6.306 \mathrm{~mol} / \mathrm{L}$. The equation is therefore:

$$
\mathrm{x}_{s}=2.8 \times 10^{-7} \frac{\mathrm{x}_{\mathrm{NaCl}}(\mathrm{T}, \rho)}{\mathrm{x}_{\mathrm{NaCl}}\left(350{ }^{\circ} \mathrm{C}, 6.3061 \mathrm{~mol} / \mathrm{L}\right)} .
$$


The uncertainty introduced by this extrapolation to the region of interest is probably at least two orders of magnitude; this is discussed more fully in a subsequent section.

It is important to reiterate that, in addition to uncertainty introduced by the extrapolation above, the poorly understood chemistry of aqueous sodium phosphate at high temperatures adds a great deal of uncertainty to these estimates. Anyone using eq (6) to estimate sodium phosphate solubility in superheated steam must recognize that the total uncertainty is probably several orders of magnitude.

\section{Sodium Sulfate}

The data situation for $\mathrm{Na}_{2} \mathrm{SO}_{4}$ at the conditions of interest is not good. Figure 13 shows the range of temperature and density covered by the data. There are a number of data points from Sastry [11] along a single isotherm at $402^{\circ} \mathrm{C}$. There are some data from the Russian literature [8, 10] reporting solubilities about an order of magnitude below Sastry in the range where they overlap. At higher temperatures, the Russian data are unusual in that they display a decrease (or at least no increase) in solubility with increasing steam density. Two points from Morey and Hesselgesser [22] at $500{ }^{\circ} \mathrm{C}$ fall two or three orders of magnitude above the Russian data. Finally, there is a single tentative datum at $500{ }^{\circ} \mathrm{C}$ from Armellini and Tester [5], who reported major difficulties in trying to establish a reproducible solubility measurement. This point is about an order of magnitude above the Russian data. Additional data on the three-phase line from the Russian thesis of Novikov [30] have a small amount of overlap with the data of Styrikovich [10]. At temperatures around $600 \mathrm{~K}$, Novikov [30] gives solubilities about two orders of magnitude below those implied by Styrikovich [10]. Near $640 \mathrm{~K}$, however (the highest temperature of his data), Novikov [30] seems to be approximately consistent with Styrikovich [10]. We consider the results of [30] to be questionable at the lower end of the reported temperature range, because the reported vapor concentrations at temperatures below about $600 \mathrm{~K}$ are so small that it is unlikely they could have been directly measured experimentally. We cannot tell from the material available to us whether some (or perhaps even all) of these vapor concentrations were somehow estimated rather than measured, but it seems that some of them must have been.

We can get an additional estimate of steam solubility of $\mathrm{Na}_{2} \mathrm{SO}_{4}$ at saturation by using the solubility in the liquid and the vapor-liquid distribution data of Goodstine [29], just as was done for sodium phosphate. Unfortunately, there is some discrepancy in the liquid solubility measurements at high temperatures. The data of Ravich and Borovaya [31], Schroeder et al. [32], and Booth and Bidwell [33] are all in fair agreement, but the solubilities of Khaibullin and Novikov $[34,35]$ are lower by a factor of 2 or 3 . We decided to accept the majority of the investigations and to begin with the saturated-liquid solubility of $2.8 \times 10^{-3}$ at $360{ }^{\circ} \mathrm{C}$ as measured by Booth and Bidwell [33]. Interpolation of the data of Goodstine [29, supplemented by additional data courtesy of $\mathrm{ABB}$ Combustion Engineering] produces a vapor-liquid distribution ratio at those conditions of $5.3 \times 10^{-4}$, leading to a solubility in the saturated vapor at $360{ }^{\circ} \mathrm{C}$ (where, for future reference, we note that the steam density is $7.9876 \mathrm{~mol} / \mathrm{L}$ ) of $1.5 \times 10^{-6}$. This number seems to be in fair agreement with extrapolations of the steam solubilities reported by Sastry [11] and by Armellini and Tester [5]; it is clearly higher than indicated by the Russian data [8, 10,30] and lower than indicated by the data of Morey and Hesselgesser [22]. 
We therefore accept this point and assume a density and temperature dependence identical to that for sodium chloride. Using the equation for sodium chloride, we then have:

$$
\mathrm{x}_{\mathrm{s}}=1.5 \times 10^{-6} \frac{\mathrm{x}_{\mathrm{NaCl}}(\mathrm{T}, \rho)}{\mathrm{x}_{\mathrm{NaCl}}\left(360^{\circ} \mathrm{C}, 7.9876 \mathrm{~mol} / \mathrm{L}\right)} \text {. }
$$

This procedure can be tested by seeing how well eq (7) agrees with the sparse and scattered data for sodium sulfate solubility (only densities below $10 \mathrm{~mol} / \mathrm{L}$ are considered here). Figures 14 and 15 show the deviations of the data from eq (7) as a function of density and temperature. We see large deviations in both directions, indicative of the scatter in the data. The largest errors are at low temperatures and densities from Novikov [30], where the equation gives solubilities several orders of magnitude higher than the data. As already mentioned, we have reason to question these measurements; it also seems unlikely that the solubilities would deviate that drastically from the much better-established values for the solubilities of $\mathrm{NaCl}$ on the vapor side of the three-phase line. The other Russian data $[8,10]$ give higher solubilities than eq (7) at low densities but lower at high densities; we have already mentioned that the density dependence of these solubility data seems physically unrealistic. The solubilities of Morey and Hesselgesser [22] are also far above that given by eq (7), but they disagree as severely with the measurements of others in the region where they overlap. Figures 16 and 17 show two approximate isotherms where eq (7) is compared to the solubility data. The equation is at least in the neighborhood of data from [5], [8], [10], and [11]. The density dependence of [8] and [10] is not reproduced, but, as mentioned above, we suspect the density dependence reported in those papers may be unphysical.

After this project was completed, we learned of the work of Pritchard et al. [36]. While they did not report their original data, they gave an equation (as a function of density only, which is physically unrealistic) correlating their solubility measurements in the range 623 to $773 \mathrm{~K}$ and 4 to $17 \mathrm{MPa}$. Their solubilities are lower than those reported by others in the same region, and are lower than and have a weaker density dependence than eq (7). Figures 16 and 17 also show the values given by [36]. This additional discrepant data, along with their observation of hydrolysis, reinforces our point that the solubility of sodium sulfate in steam is poorly understood.

In summary, eq (7) is probably the best we can do for sodium sulfate given the uncertain state of the data. It roughly agrees with the scattered measurements to within one or two orders of magnitude, with the exception of some data that we have reason to suspect. We also note that the measurement difficulties and inconsistencies could be an indication that hydrolysis (known to occur at these conditions) or other chemistry is playing a major role; for example, the bisulfate might be a significant or even dominant species in some of the experiments. These complications are beyond the scope of this study, but would add even more uncertainty to the results. The potential importance of sodium sulfate in steam power systems is such that further experimental work on this system could be very useful to industry. 


\section{Copper Oxides}

There are very few data concerning the solubility of copper oxides in steam. Figure 18 shows the range of temperature and density covered by the data in the region of interest. The solubility of $\mathrm{CuO}$ in steam has been measured by Pocock and Stewart [37] and by Hearn et al. [38]. Unfortunately, in the regions where the studies overlap, the solubilities reported by Hearn et al. are higher by more than an order of magnitude. Hearn et al. point to three factors which might cause the results of Pocock and Stewart to be systematically low: (1) there was corrosion of Pocock and Stewart's stainless steel apparatus, as evidenced by their observation of hydrogen generation, which might lead to contamination with iron-copper complexes; (2) the experimental configuration might not have allowed all of the steam to equilibrate with the solute; and (3) Hearn et al. observed that, in one modification of their own apparatus that used some stainless steel components, copper metal plated out on the stainless steel, perhaps by some electrochemical displacement reaction, giving low solubilities. Since Pocock and Stewart used a stainless steel apparatus, it is likely that this problem occurred in their experiments.

We therefore begin by fitting the data of Hearn et al. [38]. The small amount of data at low densities suggests a strategy of picking one point as a base and using the $\mathrm{NaCl}$ equation to predict other values. When this is done, the temperature dependence of the data is reproduced fairly well, but the density dependence seems to be far off. We therefore refit eq (4) to the data (specifically, just to those points at densities below $10 \mathrm{~mol} / \mathrm{L}$ ), keeping the temperature dependence determined for $\mathrm{NaCl}$ but allowing the other parameters to vary. This resulted in the equation

$$
\ln \left(\mathrm{x}_{\mathrm{S}}\right)=\mathrm{A}+\mathrm{B} / \mathrm{T}+\left(\mathrm{m}_{1}+\mathrm{m}_{3} / \mathrm{T}\right) \ln \rho-\ln \mathrm{P},
$$

with values of $\mathrm{A}=-9.75084, \mathrm{~B}=-4852.47, \mathrm{~m}_{1}=1.14584$, and $\mathrm{m}_{3}=-0.0013$. The units are the same as for eq (4); note also that the $\mathrm{B}$ and $\mathrm{m}_{3}$ parameters are unchanged. Figures 19 and 20 show the deviations of the data from eq (8) as a function of density and temperature. The Hearn [38] data (including those at densities above $10 \mathrm{~mol} / \mathrm{L}$ where eq (8) is an extrapolation) are fitted very well. The extrapolation of eq (8) also is in fair agreement with two data points at higher density from Var'yash [39]; these data are discussed further below.

However, for making a conservative estimate, eq (8) may not be sufficient. Even though we have reason to believe there are problems with the data of Pocock and Stewart [37], the Hearn et al. data are not necessarily correct. In particular, the density dependence raises a question. The data of Hearn et al. [38] showed a much weaker decrease in solubility with decreasing density than the $\mathrm{NaCl}$ data, as reflected in the need to refit the density dependence in the fitting equation. The data of Pocock and Stewart, which may be systematically low, show a somewhat faster decrease in solubility (though still not as fast as $\mathrm{NaCl}$ ). We cannot rule out the possibility that the solubility may decrease somewhat faster with decreasing density than would be indicated by extrapolating eq (8). For the low (near $0.5 \mathrm{~mol} / \mathrm{L}$ ) densities of most interest in this work, this could result in overprediction of the solubility by one or two orders of magnitude. 
In order to provide what might be a lower bound on the solubility, we also fit the Pocock and Stewart [37] data. Again, the temperature dependence from eq (4) was kept, so only the constant term and density dependence were adjusted to the data. The resulting equation is

$$
\ln \left(\mathrm{x}_{\mathrm{S}}\right)=\mathrm{A}+\mathrm{B} / \mathrm{T}+\left(\mathrm{m}_{1}+\mathrm{m}_{3} / \mathrm{T}\right) \ln \rho-\ln \mathrm{P},
$$

with values of $\mathrm{A}=-14.9399, \mathrm{~B}=-4852.47, \mathrm{~m}_{1}=2.59618$, and $\mathrm{m}_{3}=-0.0013$. The units are the same as for eq (4); again the $B$ and $m_{3}$ parameters are unchanged. Figures 21 and 22 show the deviations of the data from eq (9) as functions of density and temperature.

Unfortunately, when extrapolated to the low densities of interest in this study, eqs (8) and (9) differ by about two or three orders of magnitude (this can be seen in the sample calculations in Appendix E). This is due both to the higher solubility of the Hearn [38] data in the region where the two studies overlap, and to the larger density dependence of the solubility in the Pocock and Stewart [37] study. It is, of course, possible that the truth lies somewhere between the two data sets; for example, the Hearn data might be of the correct magnitude but the true density dependence might be that of Pocock and Stewart. It seems plausible that, in the low-density region of interest, we can consider eqs (8) and (9) to be approximate upper and lower bounds, respectively, on the solubility of $\mathrm{CuO}$.

It is worth examining the limited $\mathrm{CuO}$ solubility data at higher densities of Var'yash [39] to see if they can offer any insight into the relative merits of the other two data sets. Palmer et al. [40] recently pointed out that, when a fit they had done to the Pocock and Stewart [37] data was extrapolated to the condition of saturated liquid water at $350^{\circ} \mathrm{C}$, it came close to matching the solubility at those conditions reported by Var'yash. However, the highest water density in the data of Pocock and Stewart [37] is about $7.3 \mathrm{~mol} / \mathrm{L}$ (compared to almost $32 \mathrm{~mol} / \mathrm{L}$ for the saturated liquid data point from [39]), and the lowest temperature in [37] is about $476{ }^{\circ} \mathrm{C}$. This makes the extrapolation in both density and temperature so large that, while its results are interesting and somewhat suggestive, it cannot be given much weight as evidence for the validity of the low-density data of Pocock and Stewart [37]. This is underscored by the fact that (as seen in figures 19 and 20) our extrapolation of the Hearn [38] data as fit by eq (8) is also in reasonable agreement with this datum.

We feel that a better comparison for our purposes can be made with the point reported by Var'yash [39] at $450{ }^{\circ} \mathrm{C}$ and $50 \mathrm{MPa}$. This is within the temperature range of [38], and just below that of [37]. The density is still too high (about $22.3 \mathrm{~mol} / \mathrm{L}$ ), but not as much so as the $350{ }^{\circ} \mathrm{C}$ saturated liquid data point. Unfortunately, this point also does not help distinguish between the two data sets, because of their different density dependence. While the solubilities of Pocock and Stewart [37] are lower than those of Hearn et al. [38] at the densities where the studies overlap, they increase more quickly with density. As a result, when extrapolated, they both produce values that are in reasonable agreement with this data point of Var'yash [39]. The extrapolation of [38] comes closer to the datum, but the difference is not significant in light of the uncertainties in the data. Figure 23 shows an isotherm for $\mathrm{CuO}$ at temperatures near $740 \mathrm{~K}$, showing the behavior of eqs (8) and (9) at lower densities than those where data exist and also at the higher density of the steam solubility data point of Var'yash [39]. This figure shows the 
irreconcilable differences between the two data sets, the worsening of that difference at low density, and also the fact that the higher-density data from Var'yash [39] does not help us distinguish between the two sets.

The only $\mathrm{Cu}_{2} \mathrm{O}$ data are from Pocock and Stewart [37], and if the critique of their $\mathrm{CuO}$ data by Hearn et al. [38] is correct these data would probably also be systematically low. The data are all at essentially one temperature (about $893 \mathrm{~K}$ ) and cover a relatively narrow range of densities. If we accept the Pocock and Stewart data, it is reasonable to once again take the temperature dependence from the $\mathrm{NaCl}$ equation and adjust only the constant term and the density dependence to the data. The result is

$$
\ln \left(\mathrm{x}_{\mathrm{S}}\right)=\mathrm{A}+\mathrm{B} / \mathrm{T}+\left(\mathrm{m}_{1}+\mathrm{m}_{3} / \mathrm{T}\right) \ln \rho-\ln \mathrm{P},
$$

with values of $A=-21.4219, B=-4852.47, m_{1}=6.27227$, and $m_{3}=-0.0013$. Figure 24 shows the fit of eq (10) to the data of Pocock and Stewart [37]. The density dependence is steeper than that of the Pocock and Stewart [37] CuO data, and much steeper than for the Hearn [38] $\mathrm{CuO}$ data. If the density dependence were, for example, really that of Hearn's $\mathrm{CuO}$ data, eq (10) would underestimate the solubility at low-density conditions by several orders of magnitude. If the Pocock and Stewart [37] data have the same systematic problems that may have affected their $\mathrm{CuO}$ solubility data, then eq (10) would underestimate the solubility.

Finally, there may be uncertainties due to the chemistry for the copper oxides. Turbine deposits are sometimes copper metal and sometimes either or both of the two oxides [37]. This suggests that some electrochemical oxidation/reduction processes are taking place in the hightemperature water and/or steam. Such chemical processes would add a great deal of uncertainty to any estimates based on equilibrium solubility of individual oxidation states, and would also render suspect the solubility measurements themselves. Electrochemical transport mechanisms may well be very important for copper in steam, but they are beyond the scope of this study.

\section{Sodium Hydroxide}

It is more difficult to assess and model the solubility of sodium hydroxide because the equilibrium at conditions of interest would not be with a pure solid but rather with a $\mathrm{NaOH}$-rich liquid. The few sources of data in the region of interest $[11,13,14]$ all indicate that the equilibrium solubility of $\mathrm{NaOH}$ in low-density steam is approximately the same (within about a factor of 2, which is at least as good as the data) as that of $\mathrm{NaCl}$. We therefore consider it appropriate to apply the equation developed for $\mathrm{NaCl}$ also to $\mathrm{NaOH}$.

\section{Iron Oxides}

There is a fair amount of data for the solubility of iron oxides in liquid water at high temperatures [41-49]. The tiny solubility in liquid water is heavily influenced by the chemistry; many studies used hydrogen to maintain a reducing environment and some reported variations in solubility with $\mathrm{pH}$. In steam, the electrochemical solubility reactions would be more unfavorable. All indications are that the equilibrium solubility of iron oxides in neutral steam would be 
exceedingly small. We therefore did not consider iron oxides further on the grounds that the equilibrium transport in steam would be negligible and the only potential problem would be mechanical carryover, which is beyond the scope of this study.

\section{Assessment of Extrapolation}

Some assessment needs to be made with regard to the validity of using $\mathrm{NaCl}$ as a model substance for extrapolating limited solubility data for other substances. There are two different sources of error in this extrapolation procedure: (1) uncertainty in the behavior of $\mathrm{NaCl}$ itself, as evidenced by the data discrepancies mentioned above; and (2) the likelihood that the density and temperature dependence of the solubility of the other substance will not match that of $\mathrm{NaCl}$.

In order to get some idea of the second uncertainty, we examined the extrapolation behavior of both the $\mathrm{NaCl}$ equation (4) and the $\mathrm{SiO}_{2}$ equation (5) from a starting point of $350{ }^{\circ} \mathrm{C}$ and $6.306 \mathrm{~mol} / \mathrm{L}$, which was the starting point for the sodium phosphate extrapolation. The complete results are given in Appendix D. Here we mention the extrapolation to two points of interest. For an approximate "entrance" condition (where "entrance" and "exit" refer to conditions encountered in the channels of the Advanced Turbine System) of $300{ }^{\circ} \mathrm{C}$ and $1 \mathrm{~mol} / \mathrm{L}$, the ratio of the solubility at that state to that at the starting point was $5.016 \times 10^{-4}$ for the $\mathrm{NaCl}$ equation and $8.548 \times 10^{-3}$ for the $\mathrm{SiO}_{2}$ equation. For an "exit" condition of $600{ }^{\circ} \mathrm{C}$ and 0.5 $\mathrm{mol} / \mathrm{L}$, the ratios were $4.796 \times 10^{-4}$ for the $\mathrm{NaCl}$ equation and $1.338 \times 10^{-1}$ for the $\mathrm{SiO}_{2}$ equation.

From these sample calculations, we conclude that these extrapolation methods introduce a large uncertainty, particularly at lower densities. There can be two orders of magnitude difference between the $\mathrm{NaCl}$ and $\mathrm{SiO}_{2}$ equations. It is logical that different substances would behave somewhat differently as a function of temperature and density. If we view the solubility as a "hydration" reaction, the details of that reaction (such as the number of water molecules involved in the hydration) are potentially quite different depending on the nature of the solute. In general, there seems to be no way to predict the differences between solutes.

Therefore, while the lack of data leaves us with no other choice but to use this kind of extrapolation technique, we must recognize that the extrapolation is highly uncertain and should not be trusted within less than about two orders of magnitude, especially at low densities.

\section{Summary of Solubility Calculations}

For checking the implementation of the equations given in this paper, tables of calculated solubilities as a function of temperature and pressure (from 200 to $900{ }^{\circ} \mathrm{C}$ and 1 to $6 \mathrm{MPa}$ ) are given in Appendix E. The number of digits given in these tables is only for the purpose of checking calculations and is not indicative of the accuracy of the formulations. Appendix $E$ also lists the equation (and its coefficients) used to generate each table. In these calculations, density was determined from a given temperature and pressure from the Steam Tables [3]. Some of the points at $200{ }^{\circ} \mathrm{C}$ are not given because they would produce liquid densities, which are far outside the range of these correlations. In many cases, other points in the table will also be extrapolations 
far beyond the range in which the correlation should be used. Consult the sections on the individual solutes for comments on the level of confidence to place in these computations.

\section{Need for New Data}

The literature search on which this report is based was as thorough as possible. Essentially all the data on the compounds of interest in the region of interest were examined. Only for sodium chloride and silicon dioxide were there enough data for fairly definitive estimates of their solubilities in steam, and even for those solutes there were discrepancies between data sources at low steam densities. We must therefore conclude that, for most other common steam impurities, there are insufficient data for definitive engineering of steam cooling systems for combustion turbines. Steam purity recommendations are based on preventing deposition of impurities. Iron oxide can be controlled by feedwater chemistry. Copper oxides can be controlled by eliminating copper alloys from the system. Control of salts is more difficult. Both sodium phosphate and sodium sulfate can be expected to form low-melting deposits on the surface of the nickel alloys typically used in combustion turbines. These low-melting deposits can be expected to lead to hot corrosion. Margins can be added to the solubilities estimated in this report to provide conservative estimates of solubilities. Water can be made adequately pure to maintain the concentrations of the various compounds below conservative values. However, the cost of purifying water is not inconsequential, and the cost increases as the tolerable impurity concentration decreases. Therefore, good quality data for the solubility of sodium phosphate and sodium sulfate in steam would be economically beneficial. For those solutes where the solubility is complicated by hydrolysis or other reactions, better understanding of those reactions would also be beneficial.

Data entry and conversion, some data fitting, production of figures, and other support tasks on this project were performed by Craig J. Wardell. The collaboration which made this work possible was facilitated by Dan Friend. The authors thank Sergei Kiselev for his assistance with the Russian literature and Arno Laesecke for his assistance with the German literature. Don Palmer provided valuable constructive criticism, a prepublication copy of reference [40], and a copy of reference [17]. Reference [30] was obtained with the help of John Gallagher and Anneke Sengers. Malcolm Chase provided advice and prepublication data for the calculation of solute vapor pressures. ABB Combustion Engineering, through Stephen Goodstine, supplemented reference [29] by providing unpublished data related to the partitioning of sodium compounds between water and steam. 


\section{Bibliography}

References are listed in the order in which they are cited. Some data references were collected and tabulated without being mentioned in the main body of the report; these (beginning with reference [50] are listed in the order they appear in Appendix A. Finally, some additional papers were collected which were of less direct relevance to this work (for example, papers summarizing or correlating data reported elsewhere). These papers (beginning with reference [64]) are listed chronologically at the end of this bibliography.

[1] Spillner, F., Hochgespannter Wasserdampf als Lösungsmittel, Chem. Fabrik, 13(22), 405416 (1940).

[2] Galobardes, J.F., Van Hare, D.R. and Rogers, L.B., Solubility of sodium chloride in dry steam, J. Chem. Eng. Data, 26, 363-366 (1981).

[3] Klein, S.A. and Harvey, A.H., NIST/ASME Steam Properties, NIST Standard Reference Database 10, Version 2.0, Natl. Inst. Stand. Technol. (1996).

[4] Alekhin, Yu.V. and Vakulenko, A.G., Thermodynamic parameters and solubility of $\mathrm{NaCl}$ in water vapor at $300-500^{\circ} \mathrm{C}$ up to 300 bar, Geochem. Int., 25(5), 97-110 (1988).

[5] Armellini, F.J. and Tester, J.W., Solubility of sodium chloride and sulfate in sub- and supercritical water vapor from $450-550^{\circ} \mathrm{C}$ and $100-250$ bar, Fluid Phase Equilibria, 84, 123-142 (1993).

[6] Sourirajan, S. and Kennedy, G.C., The system $\mathrm{H}_{2} \mathrm{O}-\mathrm{NaCl}$ at elevated temperatures and pressures, Amer.J. Sci., 260, 115-141 (1962).

[7] Olander, A. and Liander, H., The phase diagram of sodium chloride and steam above the critical point, Acta Chem. Scand., 4, 1437-1445 (1950).

[8] Styrikovich, M.A., Khaibullin, I.Kh. and Tschvirashvili, D.G., Solubility of salts in high pressure steam, Dokl. Akad. Nauk SSSR, 100, 1123-1126 (1955).

[9] Aleinikov, G.I., et. al., Solubility of sodium chloride in a superheated vapor, Teploenergetika, $\underline{3}(12), 10-14$ (1956).

[10] Styrikovich, M.A., Solubility of salts in steam of supercritical characteristics, Teploenergetika, 4(2), 3-7 (1957).

[11] Sastry, V., Die Löslichkeit von Salzen in Hochgespanntem Wasserdampf und die Klärung der Frage der Turbinenversalzung, Dissertation: Technische Hochschule Karlsruhe (1957).

[12] Martynova, O.I. and Samoilov, Yu.F., The formation of solutions of inorganic substances in water vapour, Russ. J. Inorg. Chem., 7, 372-375 (1962). 
[13] Bell, M.J., Mravich, N.J., Pocock, F.J. and Rubright, M.M., Solids behavior in oncethrough nuclear steam systems, Proc. Amer. Power Conf., 39, 849-867 (1977).

[14] Allmon, W.E., Deposition of corrosive salts from steam, Electric Power Research Institute Research Project 1068-1 (NP-3002) (1983).

[15] Bischoff, J.L., Rosenbauer, R.J. and Pitzer, K.S., The system $\mathrm{NaCl}_{-} \mathrm{H}_{2} \mathrm{O}$ : relations of vaporliquid near the critical temperature of water and of vapor-liquid-halite from 300 to $500^{\circ} \mathrm{C}$, Geochim. Cosmochim. Acta, 50, 1437-1444 (1986).

[16] Wofford, W.T., Dell'Orco, P.C. and Gloyna, E.F., Solubility of potassium hydroxide and potassium phosphate in supercritical water, J. Chem. Eng. Data, 40, 968-973 (1995).

[17] Shvedov, D.N., Behavior of $\mathrm{NaCl}$ and $\mathrm{NaOH}$ in the two phase system: liquid-steam, unpublished report to the International Association for the Properties of Water and Steam (1994).

[18] Baierlein, H., Zur Löslichkeit von Salzen in überkritischem Wasserdampf, Dissertation: Universität Erlangen-Nürnberg (1983).

[19] Heitmann, H.G., Löslichkeit von Kieselsäure in Wasser und Wasserdampf sowie deren Einfluss auf Turbinenverkieselungen, Chem.-Ztg./Chem. Apparatur, 88, 891-893 (1964).

[20] Wendlandt, H.G., Reaktionen zwischen Oxiden und Wasser bei höheren Temperaturen und verschiedenen Dichten, Dissertation: Universität Göttingen (1963).

[21] Morey, G.W. and Hesselgesser, J.M., The solubility of quartz and some other substances in superheated steam at high pressures, Joint Research Committee on Boiler Feedwater Studies and the Power Division Annual Meeting, ASME Paper No. 50-A-73, 865-875 (1950).

[22] Morey, G.W. and Hesselgesser, J.M., The solubility of some minerals in superheated steam at high pressures, Econ. Geol., 46, 821-835 (1951).

[23] Kennedy, G.C., A portion of the system silica-water, Econ. Geol., 45, 629-653 (1950).

[24] Heitmann, H.G., Die Löslichkeit von Kieselsäure in Wasser und Wasserdampf sowie ihr Einfluss auf Turbinenverkieselungen, Dissertation: Technische Hochschule Karlsruhe (1964).

[25] Heitmann, H.G., Die Löslichkeit von Kieselsäure in Wasser und Wasserdampf, Glastechnische Berichte, $\underline{38(2), ~} 41-54$ (1965).

[26] Marshall, W.L., Two liquid phase boundaries and critical phenomena at $275-400^{\circ} \mathrm{C}$ for high temperature aqueous potassium phosphate and sodium phosphate solutions. Potential applications for steam generators, J. Chem. Eng. Data, 27, 175-180 (1982). 
[27] Wetton, E.A.M., Phase equilibria in aqueous sodium phosphate solutions at $350^{\circ} \mathrm{C}$, Power Industry Research, 1, 151-158 (1981).

[28] Schroeder, W.C., Berk, A.A. and Gabriel, A., Solubility equilibria of sodium sulfate at temperatures from 150 to 350 degrees. III. Effect of sodium hydroxide and sodium phosphate, J. Am. Chem. Soc., 모, 1783-1790 (1937).

[29] Goodstine, S.L., Vaporous carryover of sodium salts in high-pressure steam, Proc. Amer.

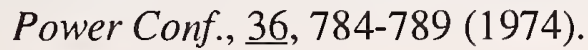

[30] Novikov, B.E., Investigation of thermodynamic properties and behavior of salts with common ions in boiler water at high temperatures, Dissertation: Moscow Power Institute (1973).

[31] Ravich, M.I. and Borovaya, F.E., Phase equilibria in the sodium sulphate-water system at high temperatures and pressures, Russ. J. Inorg. Chem., 9, 520-532 (1964).

[32] Schroeder, W.C., Gabriel, A. and Partridge, E.P., Solubility equilibria of sodium sulfate at temperatures of 150 to 350 degrees. I. Effect of sodium hydroxide and sodium chloride, $J$. Am. Chem. Soc., 57, 1539-1546 (1935).

[33] Booth, H.S. and Bidwell, R.M., Solubilities of salts in water at high temperatures, J. Am. Chem. Soc., 72, 2567-2575 (1950).

[34] Khaibullin, I.Kh. and Novikov, B.E., A thermodynamic study of aqueous and steam solutions of sodium sulfate at high temperatures, Teplofizika Vysokikh Temperatur, 11(2), 320-327 (1973).

[35] Khaibullin, I.Kh. and Novikov, B.E., Gamma-ray examination of saturation parameters and thermophysical properties of multicomponent salt systems at high temperatures, Mater. Vses. Nauchno-Tekh. Soveshch. Teploobmennym Teplofiz. Svoistvam Morsk. Solonovatykh Vod Ikh Ispol'z, 339-350 (1973).

[36] Pritchard, A.M., Peakall, K.A., Smart, E.F. and Bignold, G.J., The solubility of sodium sulfate in superheated steam between 623-773 K and 4-17 MPa, in Water Chemistry for Nuclear Reactor Systems 4, BNES, London, 233-234 (1986).

[37] Pocock, F.J. and Stewart, J.F., The solubility of copper and its oxides in supercritical steam, J. Eng. Power, 85, 33-45 (1963).

[38] Hearn, B., Hunt, M.R. and Hayward, A., Solubility of cupric oxide in pure subcritical and supercritical water, J. Chem. Eng. Data, 14, 442-447 (1969).

[39] Var'yash, L.N., Hydrolysis of $\mathrm{Cu}(\mathrm{II})$ at $25-350^{\circ} \mathrm{C}$, Geochem. Int., 23, 82-92 (1986). 
[4(1) Palmer, D.A., Simonson. J.M. and Joyce, D.B., Volatility of Copper, paper presented at International Conference on Interaction of Non-Iron Based Materials with Water and Steam, June 1996, Piacenza, Italy.

[41] Holser, W.T. and Schneer, C.J., Hydrothermal magnetite, Geol. Soc. America Bull., 72 , 369-386 (1961).

[42] Martynova, O.I, Serov, E.P., Smirnov, O.K., and Tskhvirashvilli, D.G., Solubility of iron oxides in steam of high and supercritical parameters, Izv. Akad. Nauk SSSR, Energ. $i$ Transp., 6, 759-762 (1963).

[43] Martynova, O.I., Serov, E.P. and Smirnov, O.K., Carry-over of iron oxide by superheated steam above critical pressure, Teploenergetika, 10(7), 54-57 (1963).

[44] Styrikovich, M.A., Samoilov, Yu.F. and Gryazev, A.M., The solubility of iron oxides in steam, Thermal Engineering, 15(4), 100-102 (1968).

[45] Sweeton, F.H. and Baes, C.F., Jr., The solubility of magnetite and hydrolysis of ferrous ion in aqueous solutions at elevated temperatures, J. Chem. Thermodyn., 2 2, 479-500 (1970).

[46] Styrikovich, O.I., Martynova, I.F., Kobyakov, I.F. and Men'shikova, V.L., Solubility of magnetite in boiling water at high temperature, Thermal Engineering, 18, 116-119 (1971).

[47] Styrikovich, M.A., Martynova, O.I., Kobyakov, I.F., Men'shikova, V.L. and Reznikov, M.I., The solubility of magnetite in water at high temperature, in a reducing medium, Teploenergetika, 19(9), 85-87 (1972).

[48] Chou, I. and Eugster, H.P., Solubility of magnetite in supercritical chloride solutions, Amer. J. Sci., 277, 1296-1314 (1977).

[49] Tremaine, P.R. and LeBlanc, J.C., The solubility of magnetite and the hydrolysis and oxidation of $\mathrm{Fe}(2+)$ in water to $300^{\circ} \mathrm{C}, J$. Solution Chem., $9,415-442(1980)$.

[50] Wyart, J. and Sabatier, G., Nouvelles mesures de la solubilitie du quartz, de la tridymite et de la cristobalite dans l'eau sous pression au-dessus de la temperature critique, Acad. Sci. Paris Comptes Rendus, 240, 1905-1907 (1955).

[51] Khitarov, N.I., The 400 degree isotherm for the system $\mathrm{H}_{2} \mathrm{O}-\mathrm{SiO}_{2}$ at pressures up to 2000 $\mathrm{kg} / \mathrm{cm} 2$, Geochem. Int., 1, 55-61 (1956).

[52] Kitahara, S., The solubility of quartz in water at high temperatures and pressures, Rev. Phys. Chem. Japan, 30, 109-114 (1960).

[53] Kennedy, G.C., Wasserburg, G.J., Heard, H.C. and Newton, R.C., The upper three phase region in the system $\mathrm{SiO}_{2}-\mathrm{H}_{2} \mathrm{O}$, Amer. J. Sci., 260, 501-521 (1962). 
[54] Morey, G.W., Fournier, R.O., and Rowe, J.J., The solubility of quartz in water in the temperature interval from $25^{\circ}$ to $300^{\circ} \mathrm{C}$, Geochim. Cosmochim. Acta, 26, 1029-1043 (1962).

[55] Weill, D.F. and Fyfe, W.S., The solubility of quartz in $\mathrm{H}_{2} \mathrm{O}$ in the range $1000-4000$ bars and 400-550 ${ }^{\circ}$, Geochim. Cosmochim. Acta, $\underline{28}, 1243-1255$ (1964).

[56] Anderson, G.M. and Burnham, C.W., The solubility of quartz in supercritical water, Amer. J. Sci., 263, 494-511 (1965).

[57] Semenova, A.I. and Tsiklis, D.S., Solubility of silicon dioxide in steam at high pressures and temperatures, Russ. J. Phys. Chem., 44, 1420-1422 (1970).

[58] Crerar, D.A. and Anderson, G.M., Solubility and solvation reactions of quartz in dilute hydrothermal solutions, Chemical Geology, ㅇ, 107-122 (1971).

[59] Hemley, J.J., Montoya, J.W., Marinenko, J.W. and Luce, R.W., Equilibria in the system $\mathrm{Al}_{2} \mathrm{O}_{3}-\mathrm{SiO}_{2}-\mathrm{H}_{2} \mathrm{O}$ and some general implications for alteration/mineralization processes, Econ. Geol., 75, 210-228 (1980).

[60] Ragnarsdottir, K.V. and Walther, J.V., Pressure sensitive "silica geothermometer" determined from quartz solubility experiments at $250^{\circ} \mathrm{C}$, Geochim. Cosmochim. Acta, 47, 941-946 (1983).

[61] Walther, J.V. and Orville, P.M., The extraction-quench technique for determination of the thermodynamic properties of solute complexes: application to quartz solubility in fluid mixtures, Amer. Mineralogist, 68, 731-741 (1983).

[62] Manning, C.E., The solubility of quartz in $\mathrm{H}_{2} \mathrm{O}$ in the lower crust and upper mantle, Geochim. Cosmochim. Acta, 58, 4831-4839 (1994).

[63] Urusova, M.A., Phase equilibria in the sodium hydroxide-water and sodium chloride-water systems at $350-550^{\circ} \mathrm{C}$, Russ. J.Inorg. Chem., 19, 450-454 (1974).

[64] Ravich, M.I., Borovaya, F.E. and Ketkovich, V.Ya., Solubility and saturation pressure of the solution in the system $\mathrm{NaCl}-\mathrm{Na}_{2} \mathrm{SO}_{4}-\mathrm{H}_{2} \mathrm{O}$ at high temperatures, Akad. Nauk SSSR. Institut Obshchei i Neorg. Khimii. Sektor Fiziko-Khim. Anoliza. Izvestiya, 22, 240-254 (1953).

[65] Styrikovich, M.A. and Khaibullin, I.Kh., Phase equlibrium diagrams of the systems $\mathrm{NaCl}$ $\mathrm{H}_{2} \mathrm{O}, \mathrm{Na}_{2} \mathrm{SO}_{4}-\mathrm{H}_{2} \mathrm{O}, \mathrm{CaSO}_{4}-\mathrm{H}_{2} \mathrm{O}$ and $\mathrm{SiO}_{2}$ (quartz)- $\mathrm{H}_{2} \mathrm{O}$, Dokl. Akad. Nauk SSSR, 109, $962-$ 965 (1956).

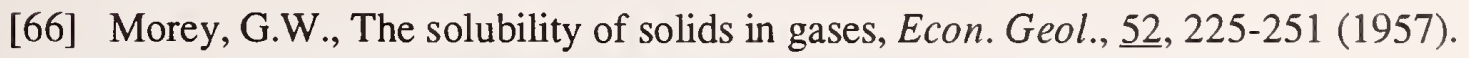

[67] Styrikovich, M.A., The solubility of low volatility substances in high pressure steam, Radioisotopes Sci. Research, 1, 411-425 (1957). 
[68] Mosebach, R., Thermodynamic behavior of quartz and other forms of silica in pure water at elevated temperatures and pressures with conclusions on their mechanism of solution, $J$. Geology, 65(4), 347-363 (1957).

[69] Khailbullin, I.Kh. and Zenkevich, Yu.V., On the nature of silica carry over with high pressure steam, Teploenergetika, No. 6, 16-20 (1958).

[70] Van Lier, J.A., De Bruyn, P.L. and Overbeek, J.Th.G., The solubility of quartz, J. Phys. Chem., 64, 1675-1682 (1960).

[71] Kot, A.A., Dejewa, S.W. and Pschemenski, A.A., Über die Löslichkeit von Quarz, Kieselsäure und Natriumsilikat in überhitztem Dampf, Arch. für Energiewirtschaft, 14, 547557 (1961).

[72] Wendlandt, H.G. and Glemser, O., Study of the reactions of oxides with water at high temperatures and pressures, Angew. Chemie, 75, 949-957 (1963).

[73] Martynova, O.I., Some problems of the solubility of involatile inorganic compounds in water vapor at high temperatures and pressures, Russ. J. Phys. Chem., $\underline{38}$, 587-592 (1964).

[74] Styrikovich, M.A., Martynova, O.I. and Mingulina, E.I., The dependence of the coordination number of weak electrolytes on the density of the solvent water, Doklady Phys. Chem., 171, $911-914$ (1966).

[75] Martynova, O.I., Solubility of inorganic compounds in subcritical and supercritical water, in High Temperature, High Pressure Electrochemistry, 4th ed., National Association of Corrosion Engineers: Houston, 131-138 (1976).

[76] Fournier, R.O. and Potter, R.W., An equation correlating the solubility of quartz in water from 25 degrees to 900 degrees $\mathrm{C}$ at pressures up to 10,000 bars, Geochim. Cosmochim. Acta, 46, 1969-1973 (1982).

[77] Von Damm, K.L., Bischoff, J.L. and Rosenbauer, R.J., Quartz solubility in hydrothermal seawater: an experimental study and equation describing quartz solubility for up to $0.5 \mathrm{M}$ $\mathrm{NaCl}$ solutions, Amer. J. Sci., 291, 977-1007 (1991).

[78] Fournier, R.O. and Thompson, J.M., Composition of steam in the system NaCl-KCl- $\mathrm{H}_{2} \mathrm{O}-$ Quartz at $600^{\circ} \mathrm{C}$, Geochim. Cosmochim. Acta, 57, 4365-4375 (1993). 


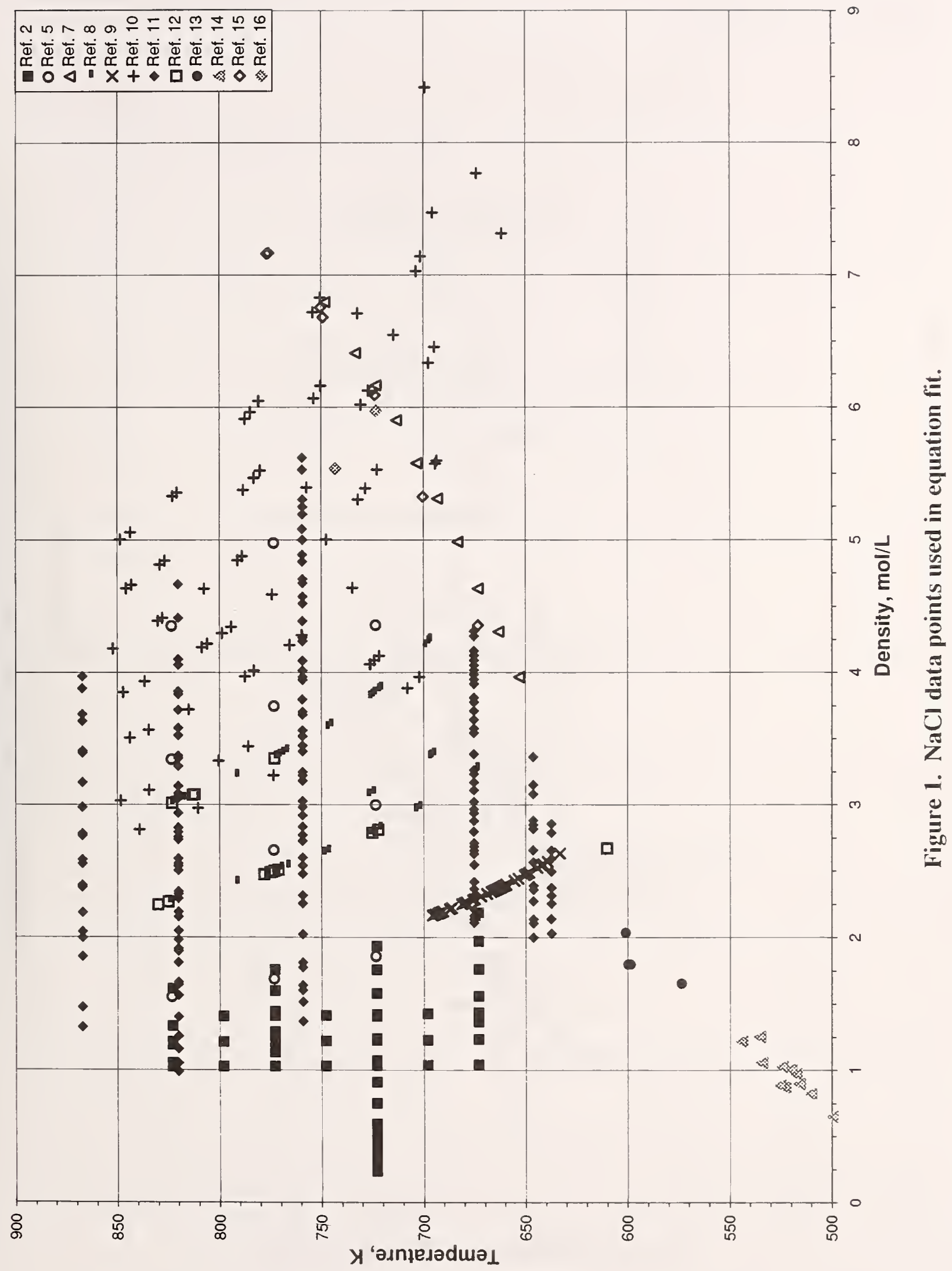




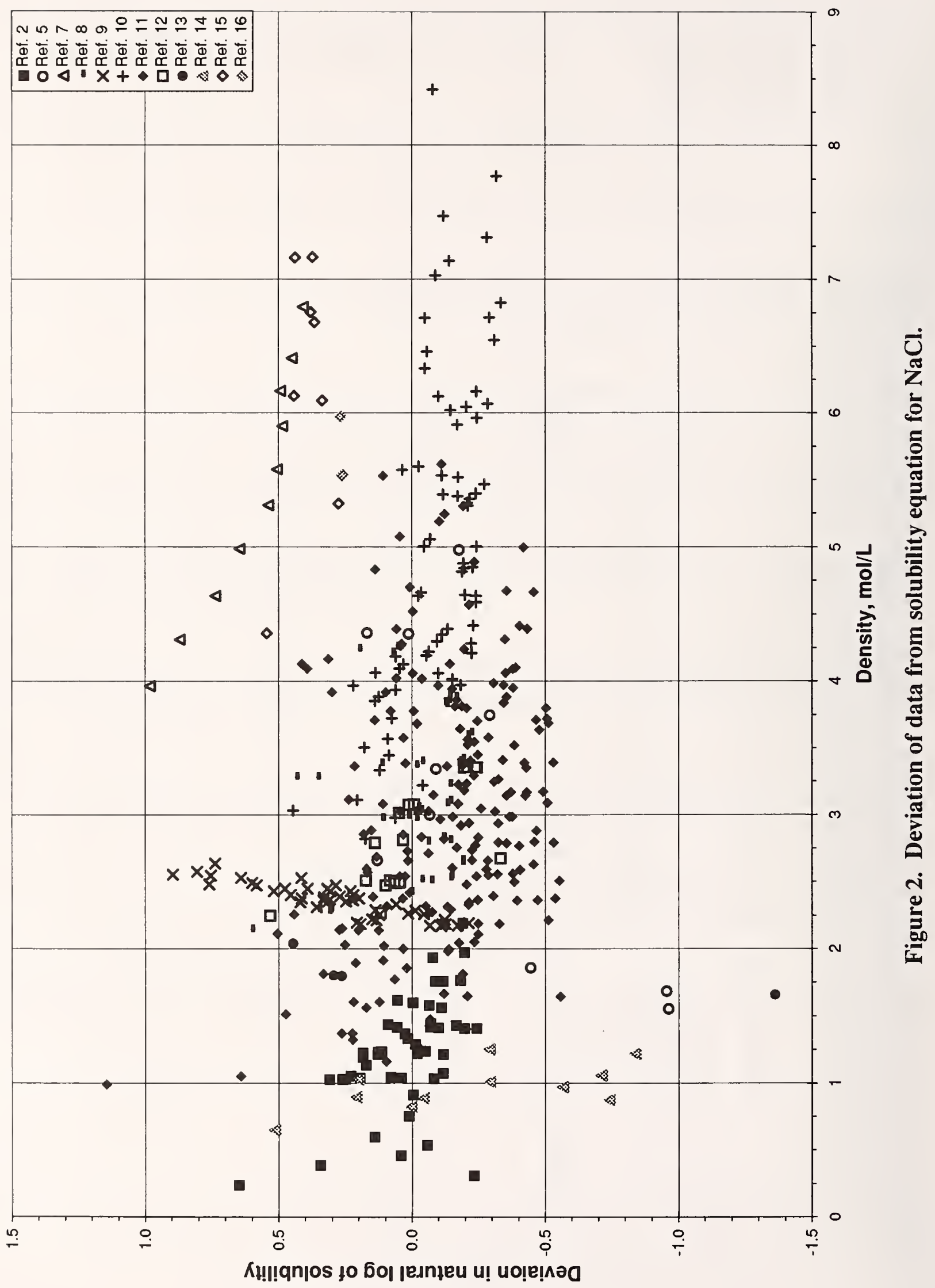




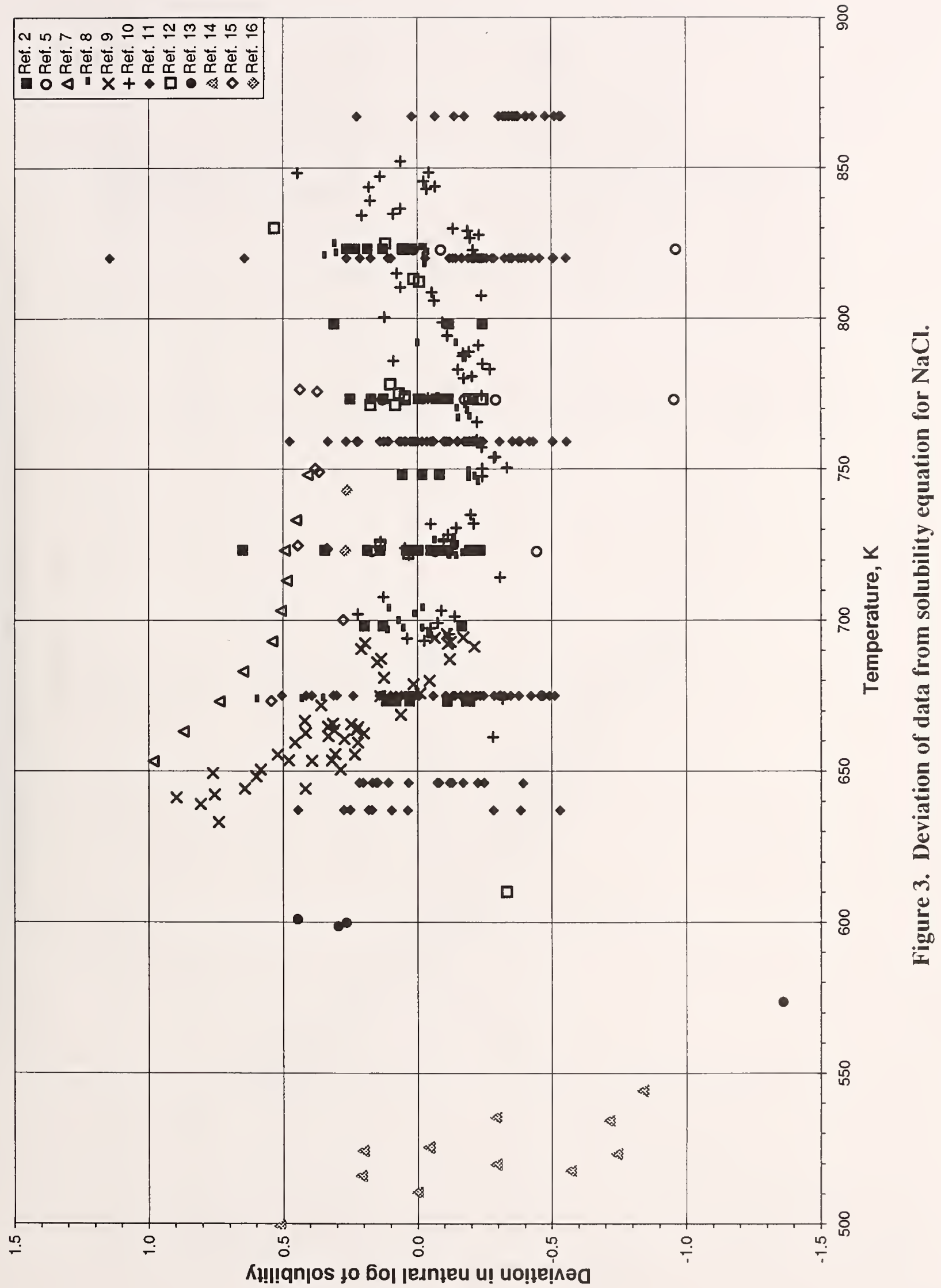




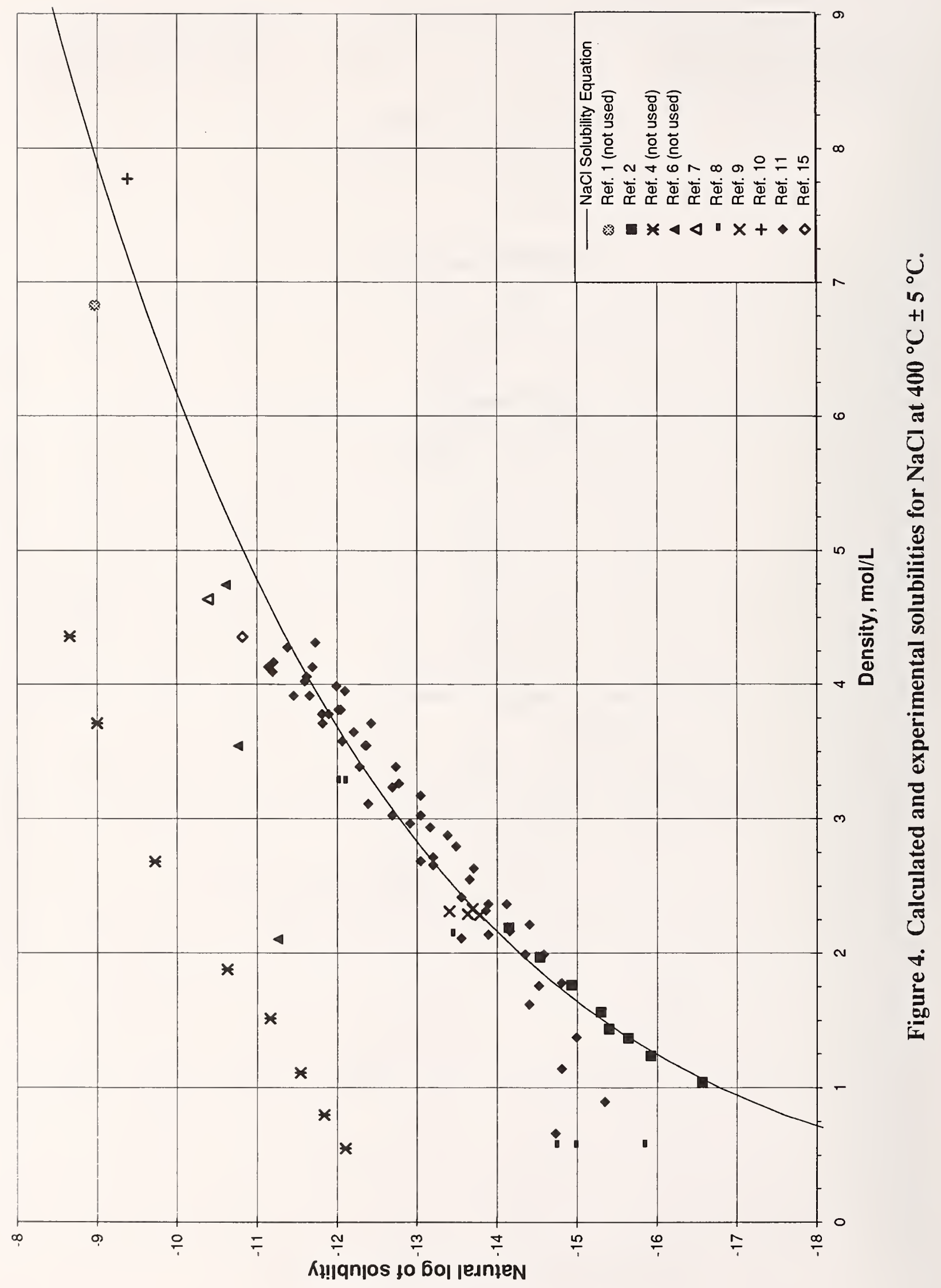




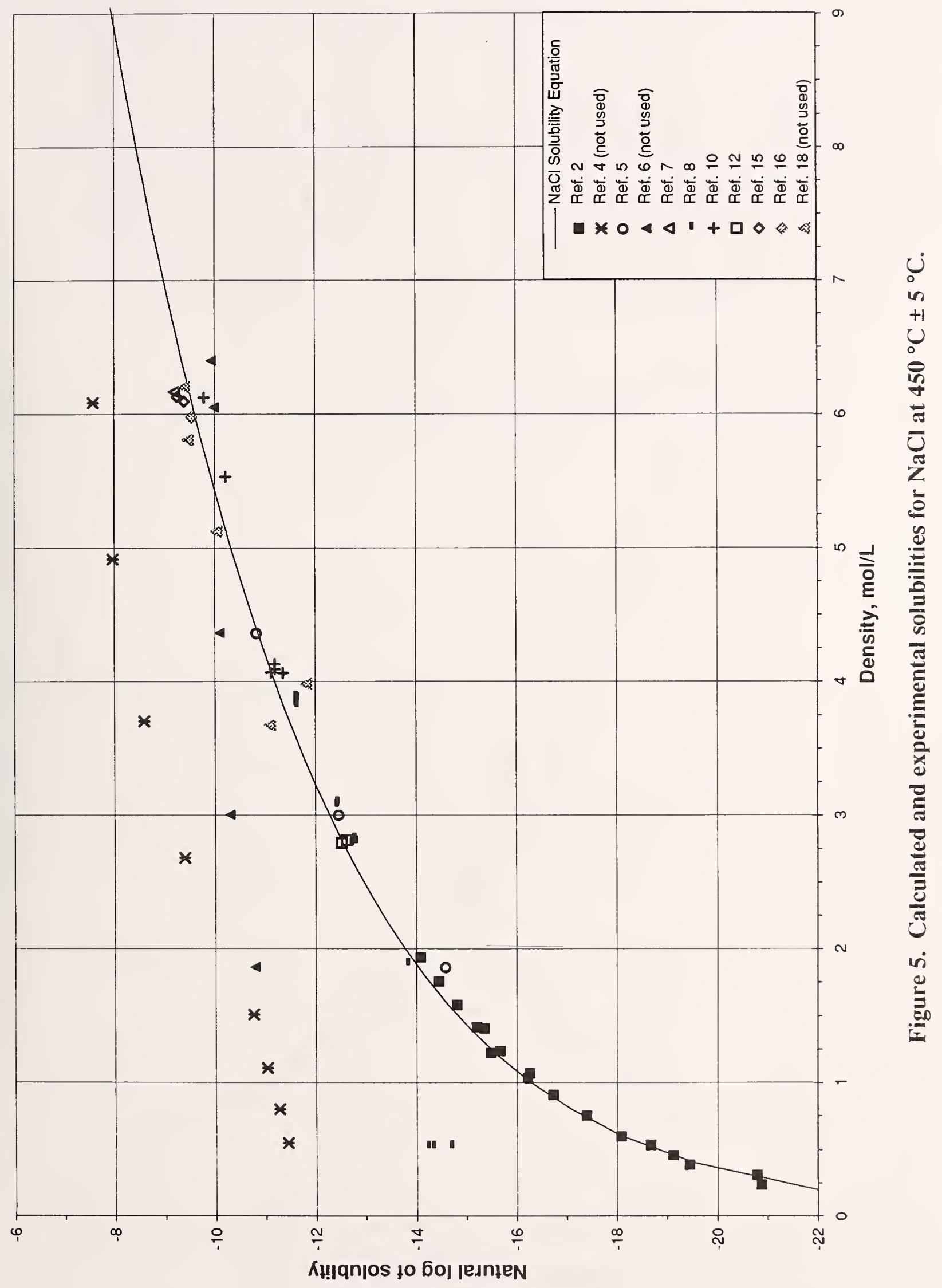




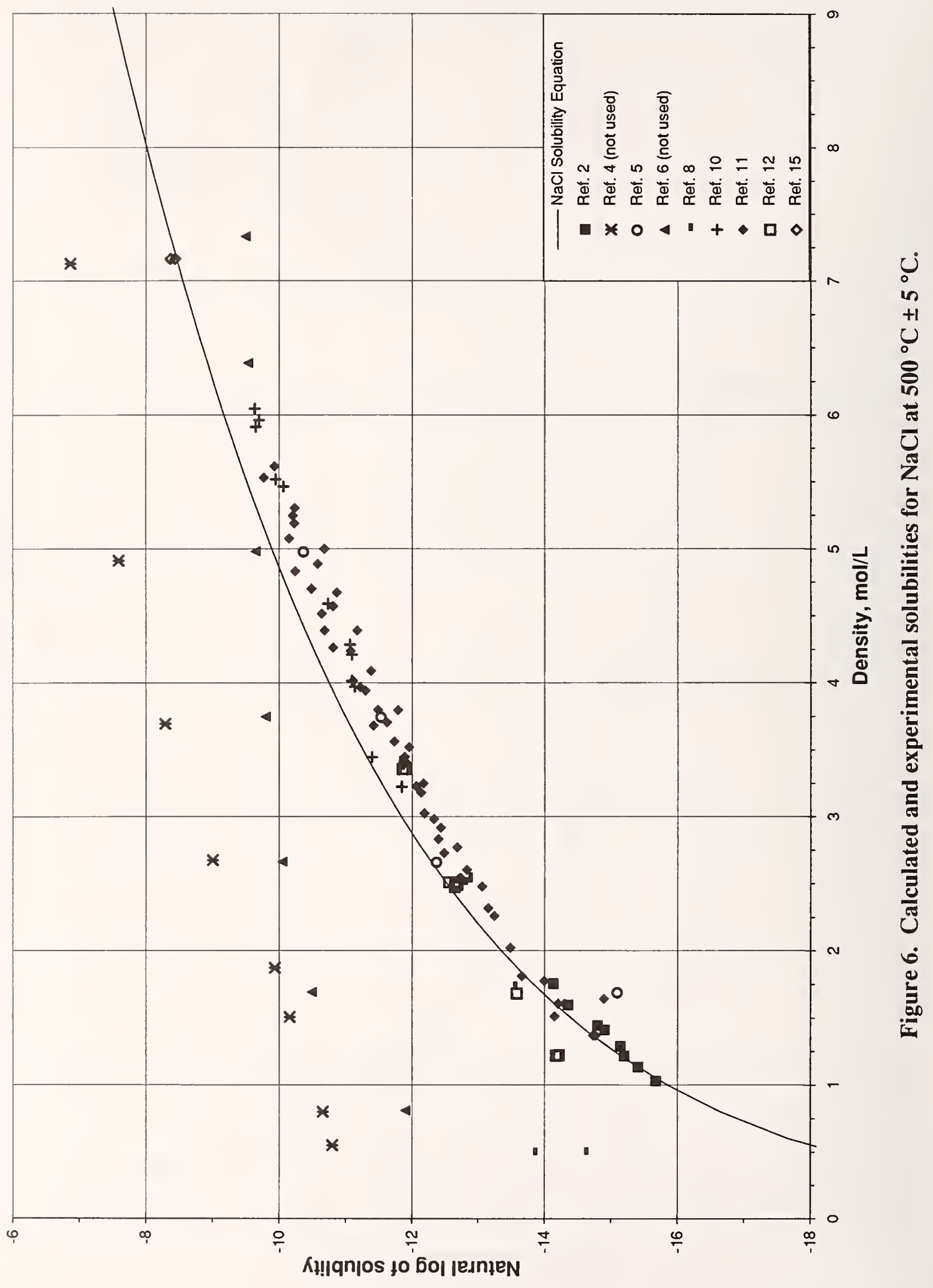




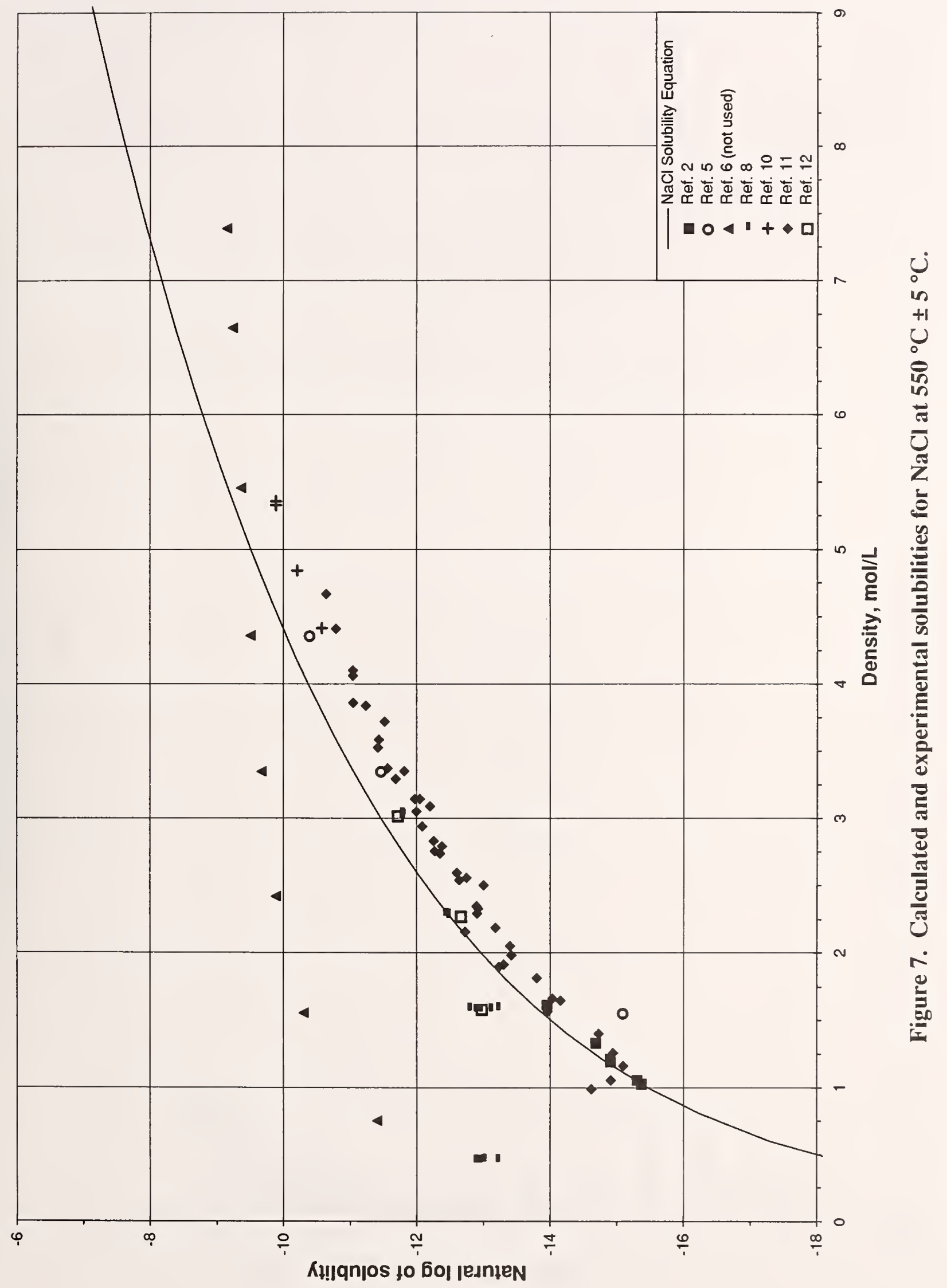




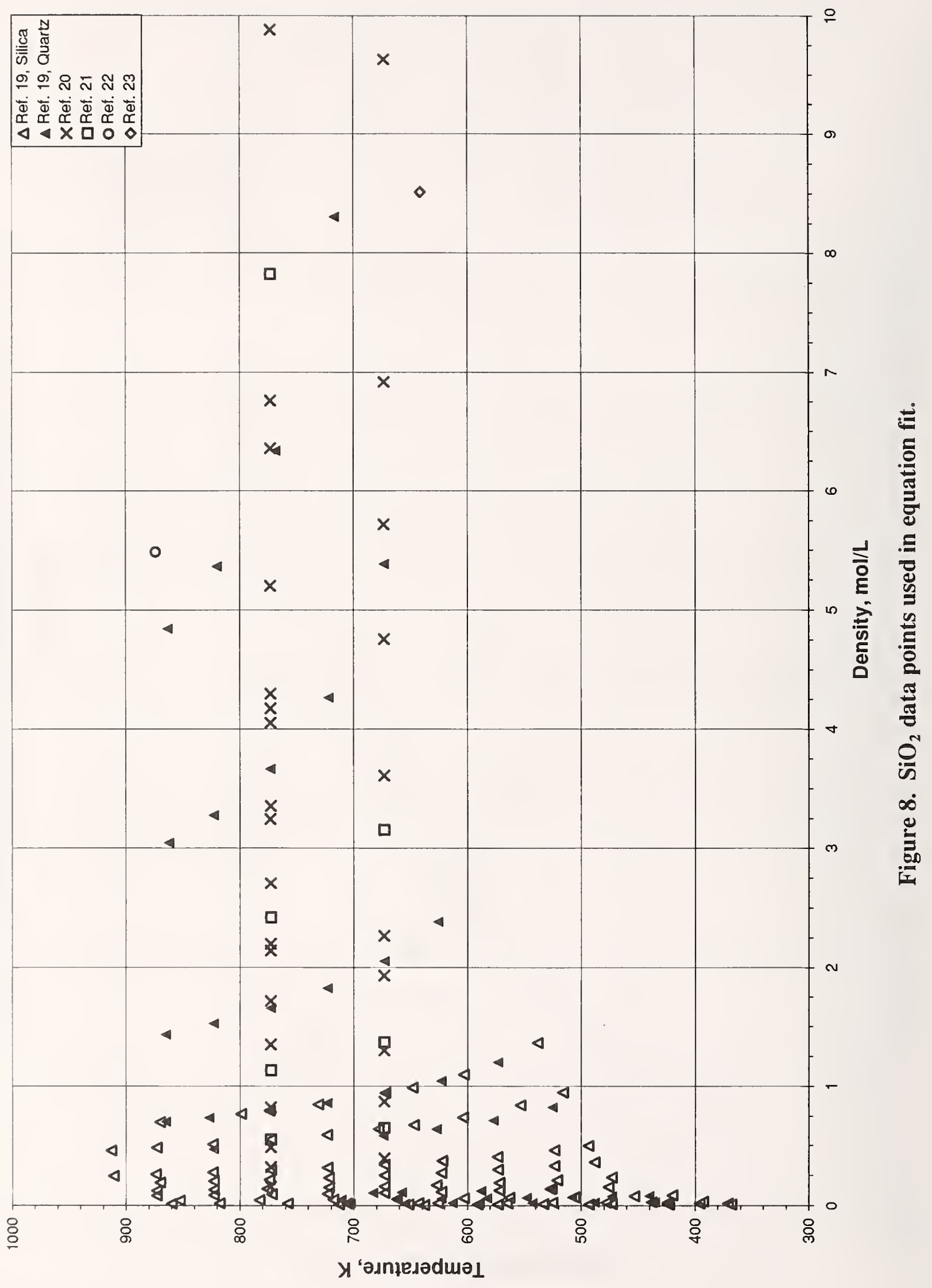




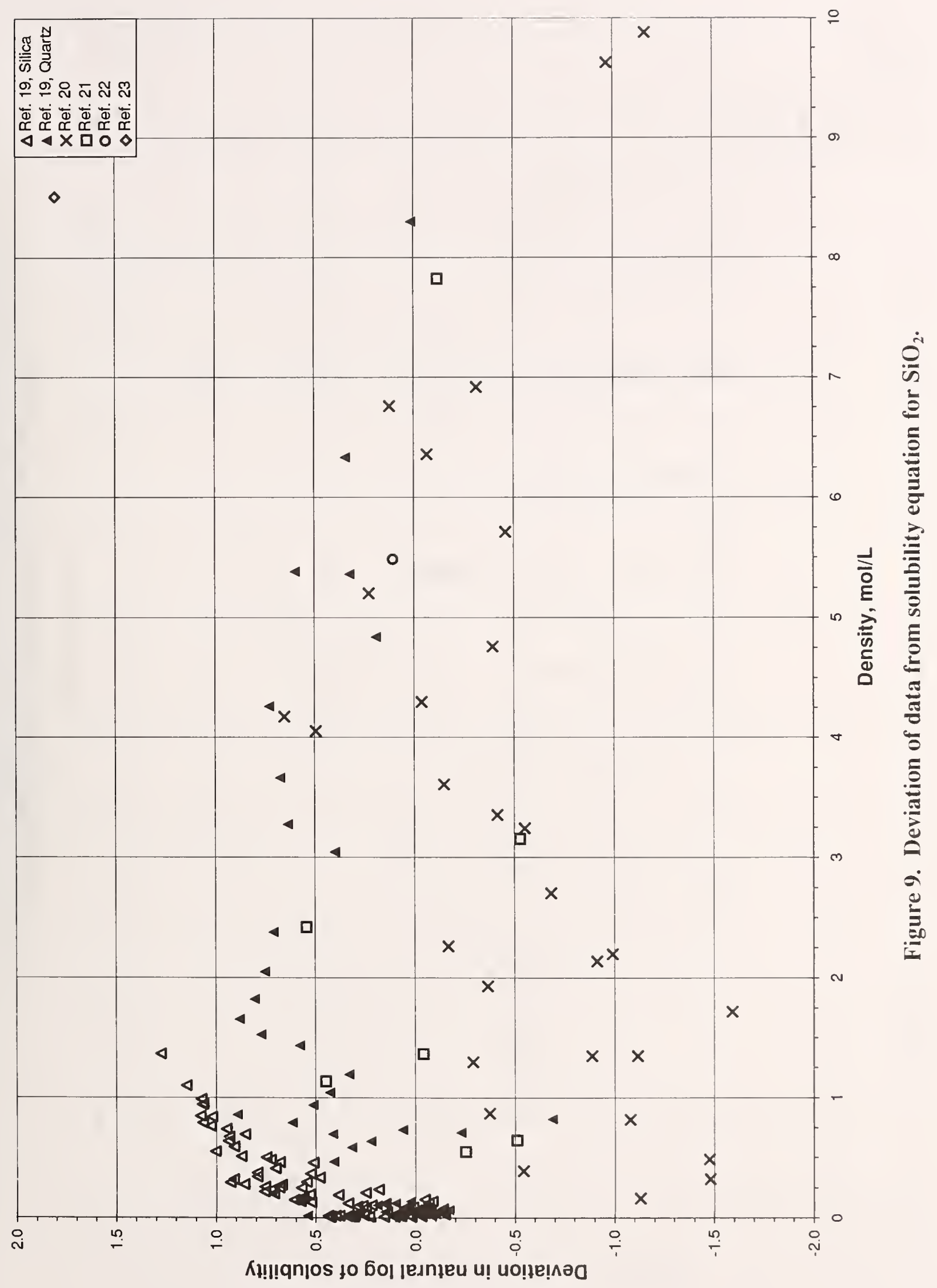




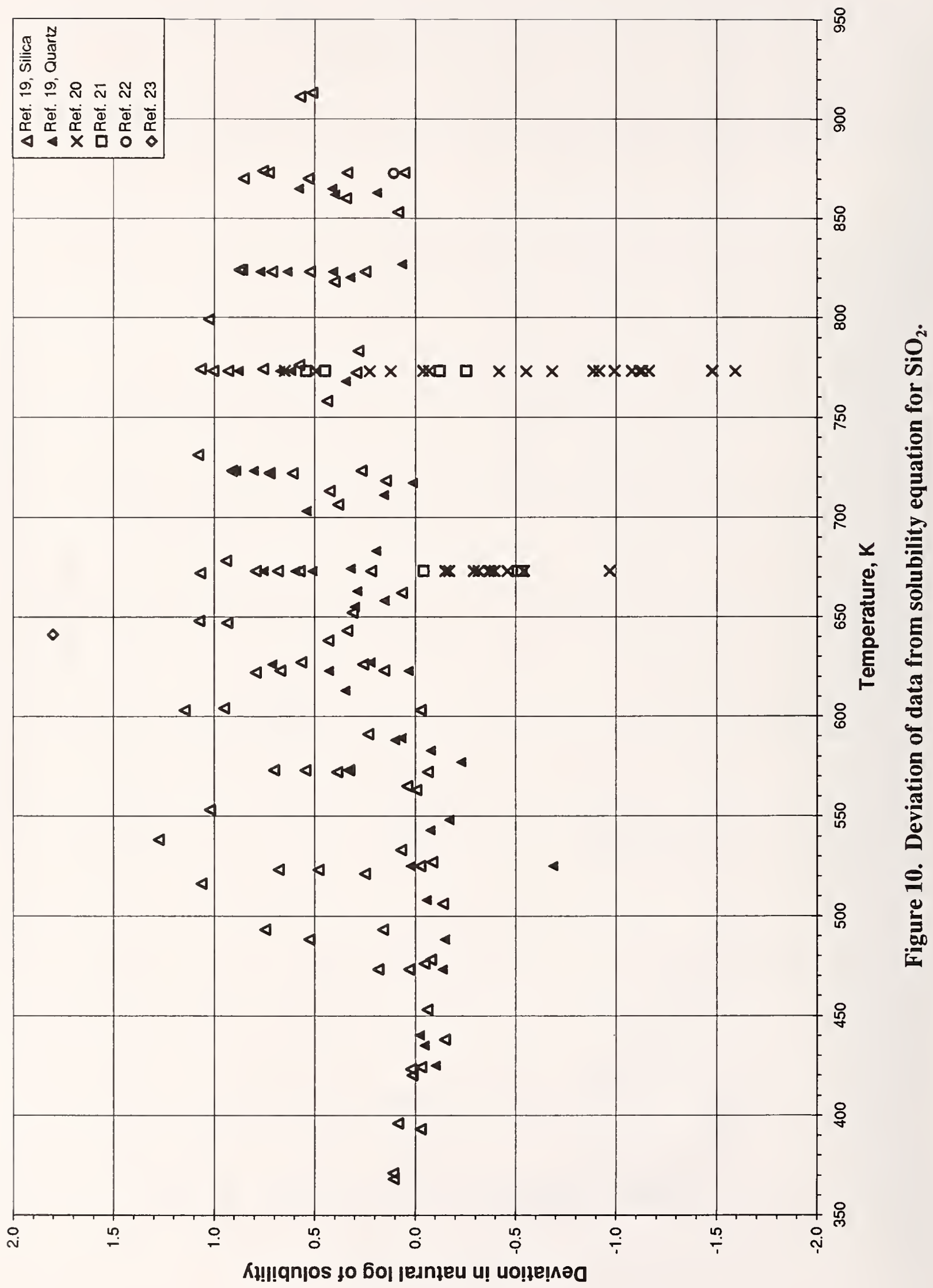




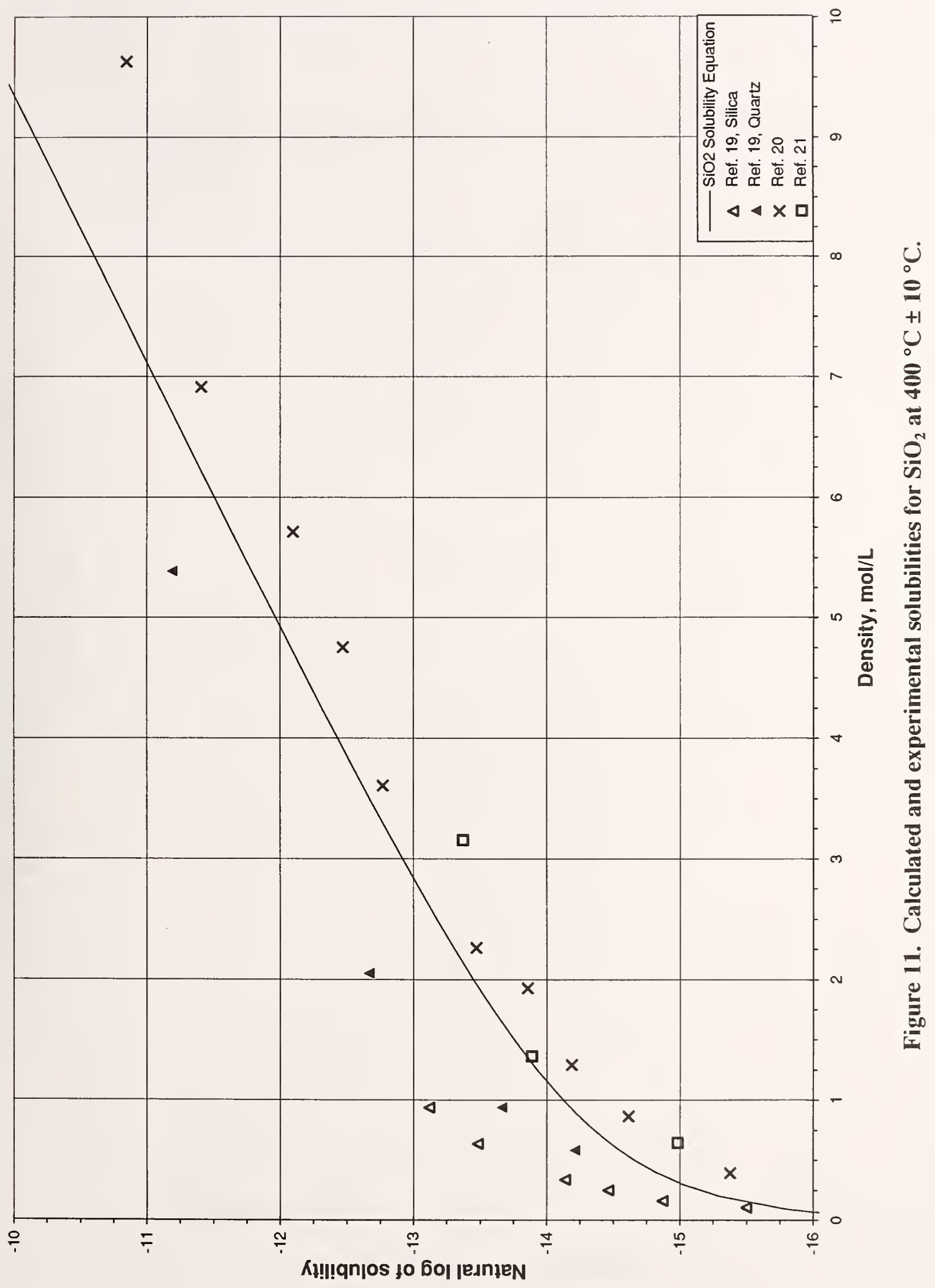




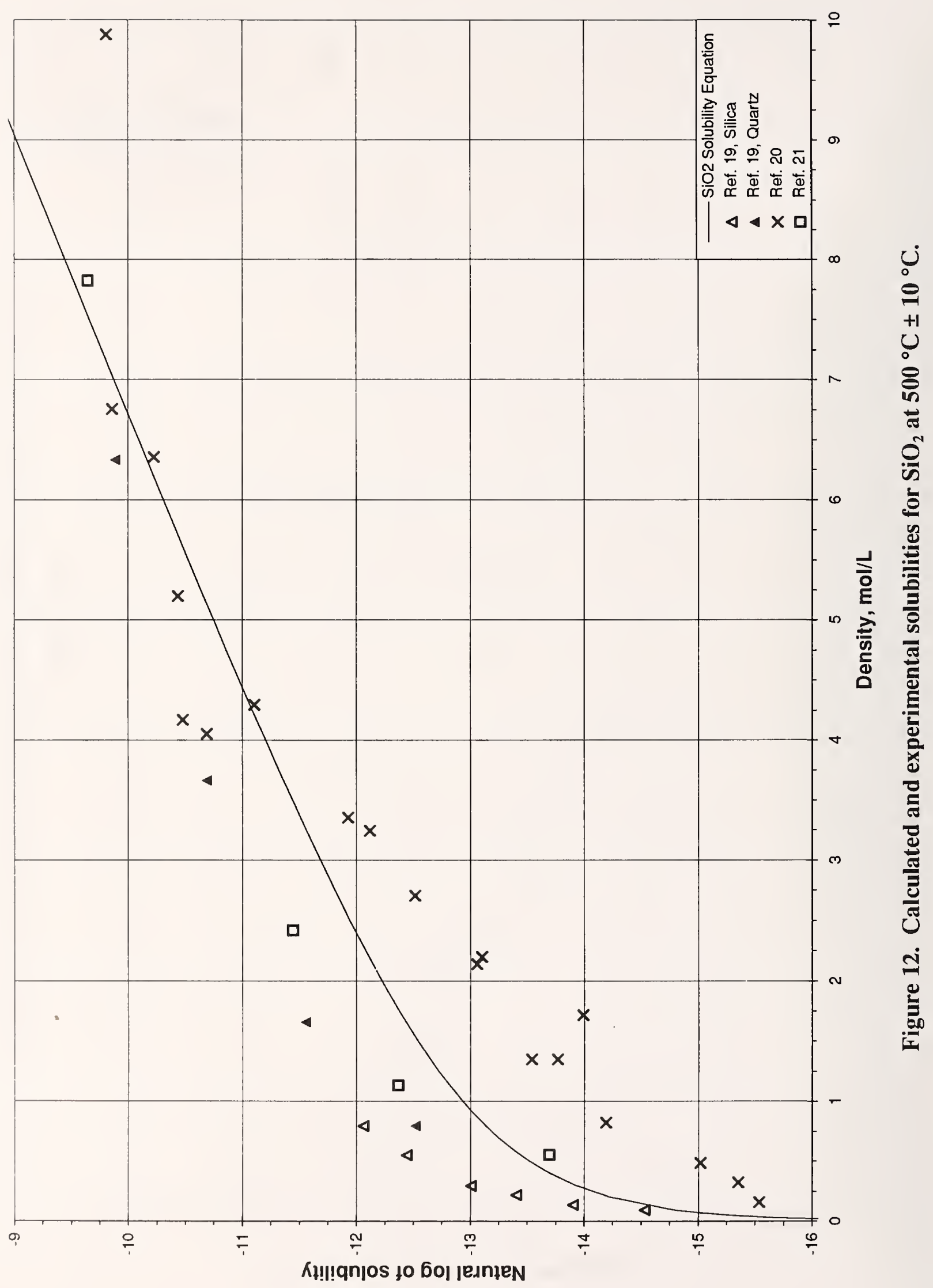




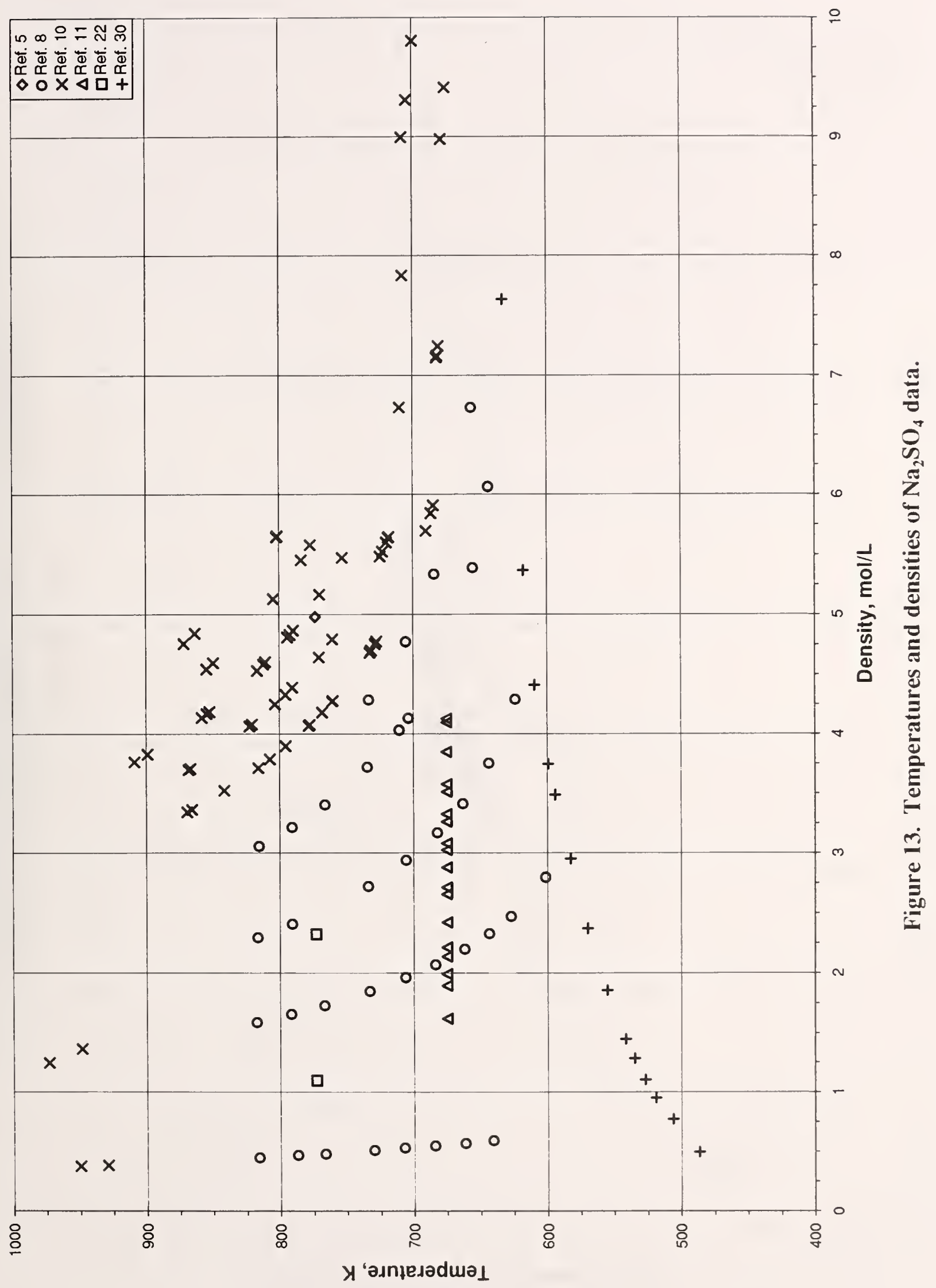




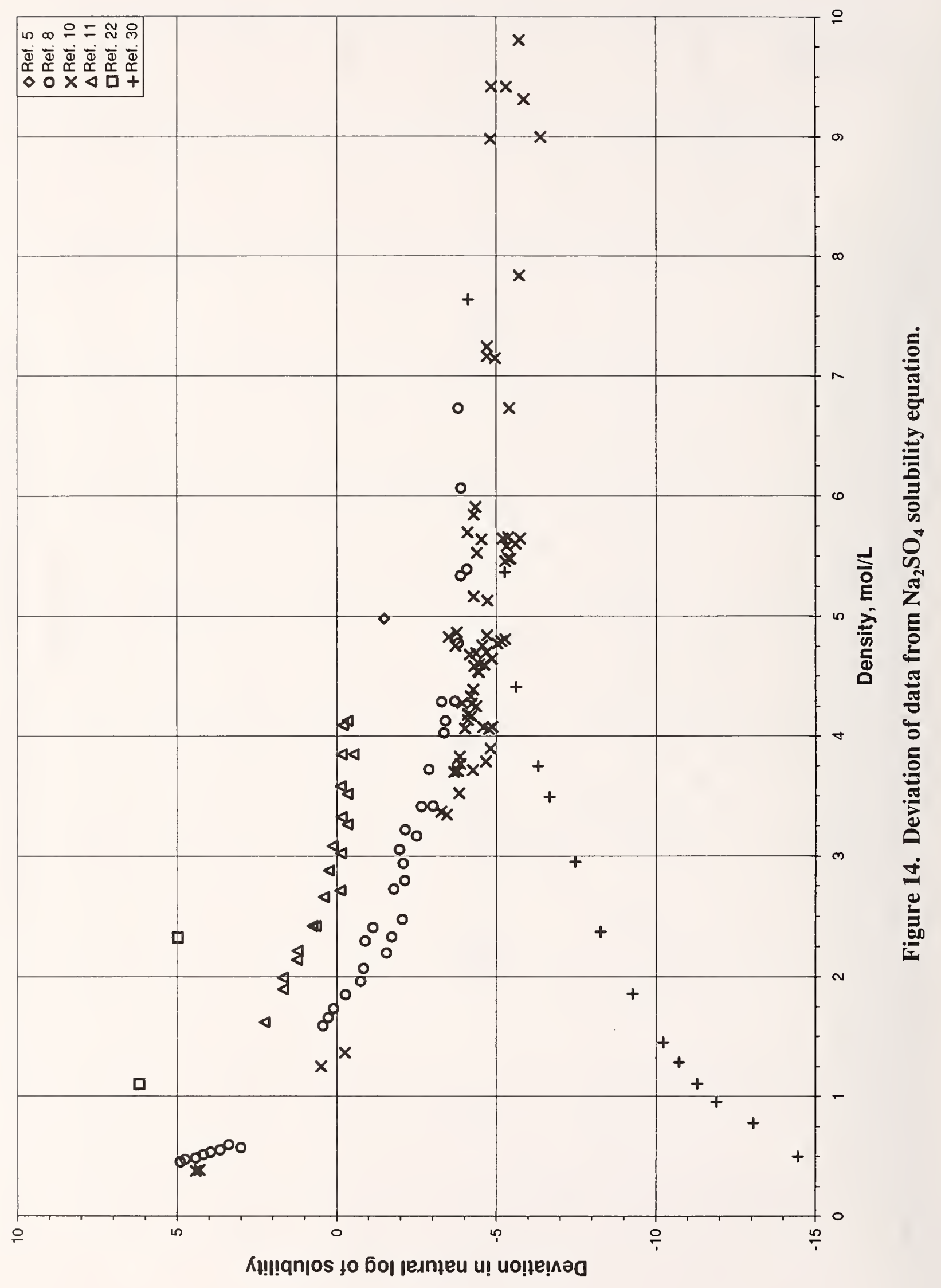




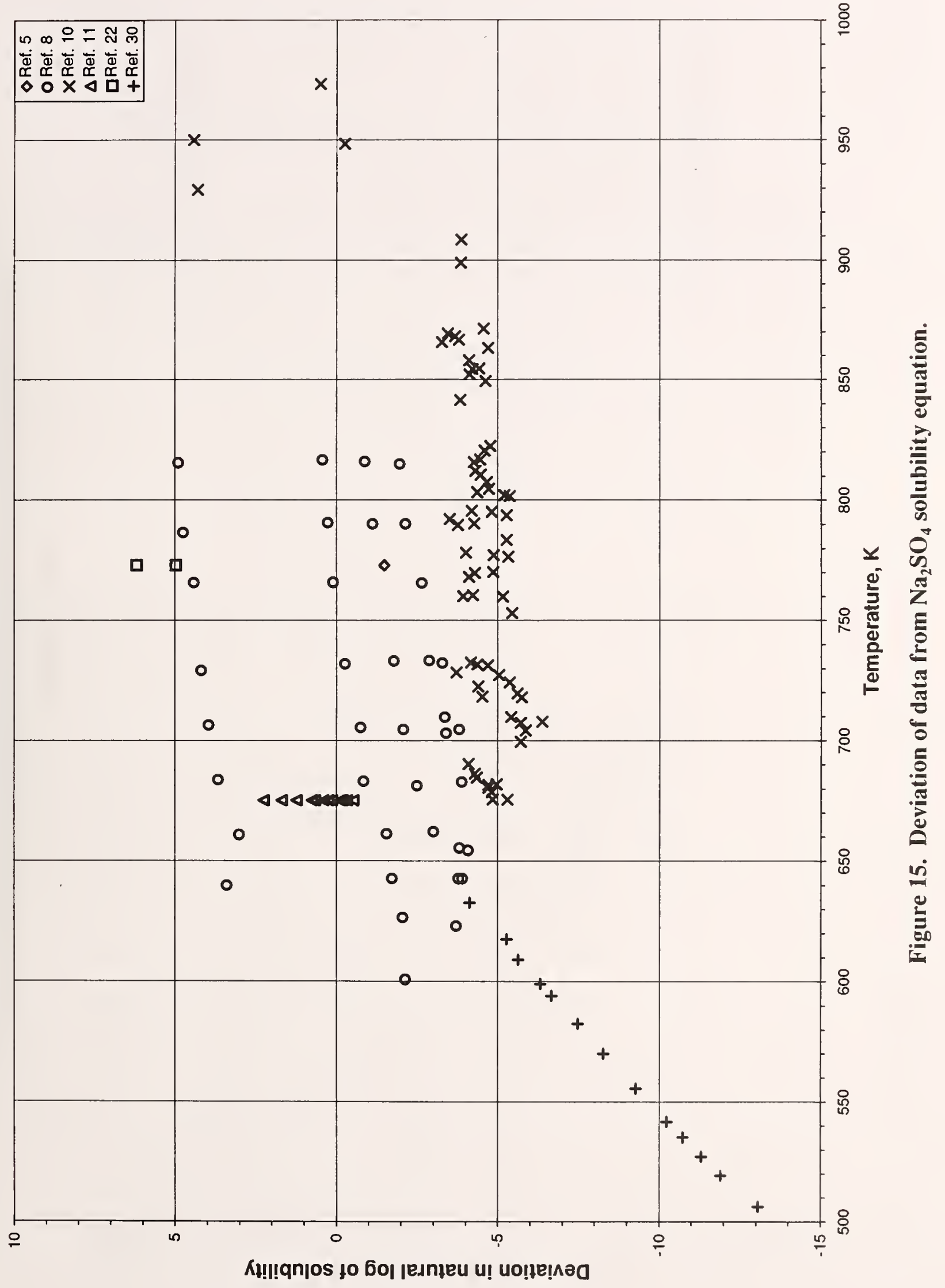




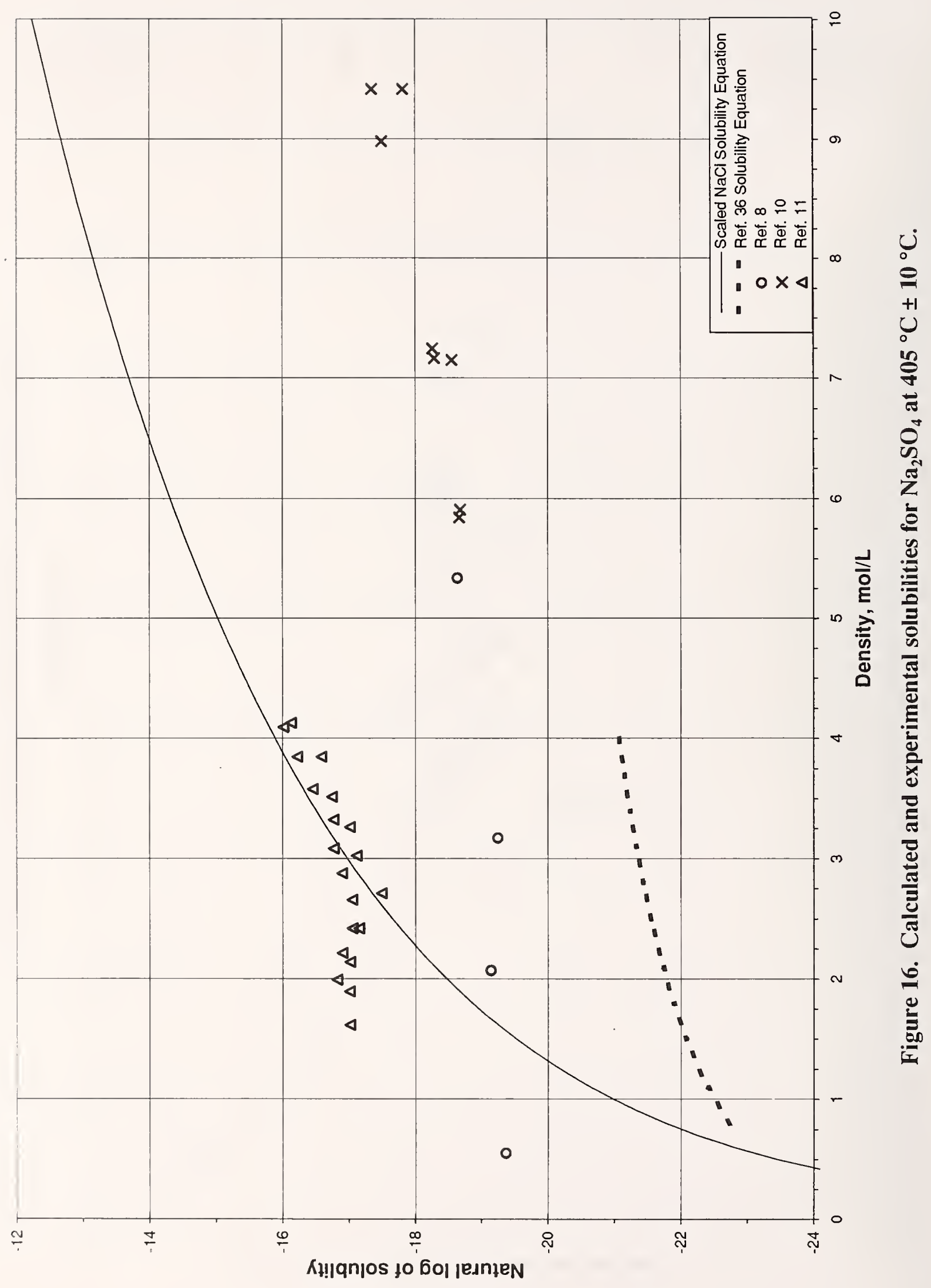




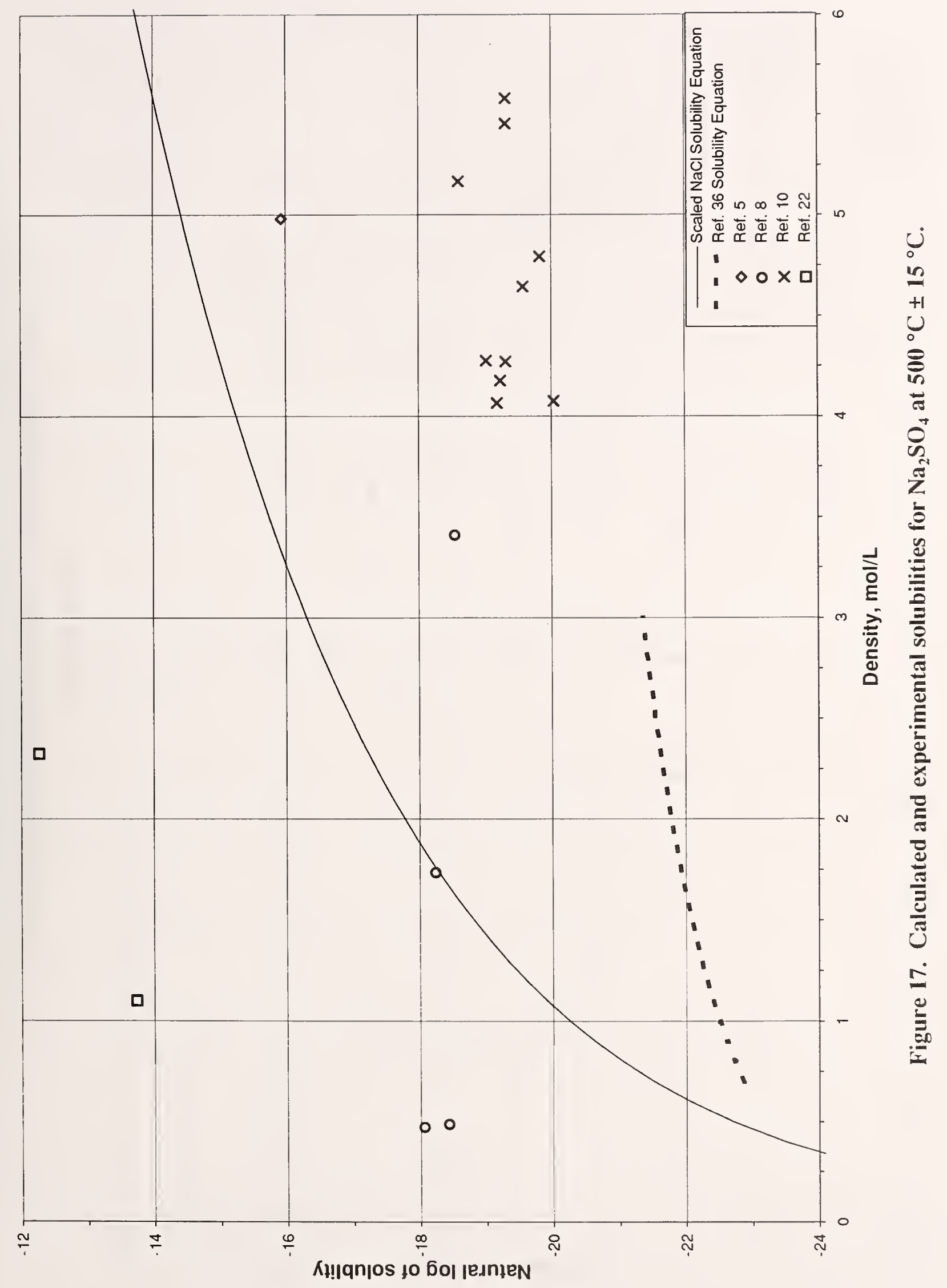




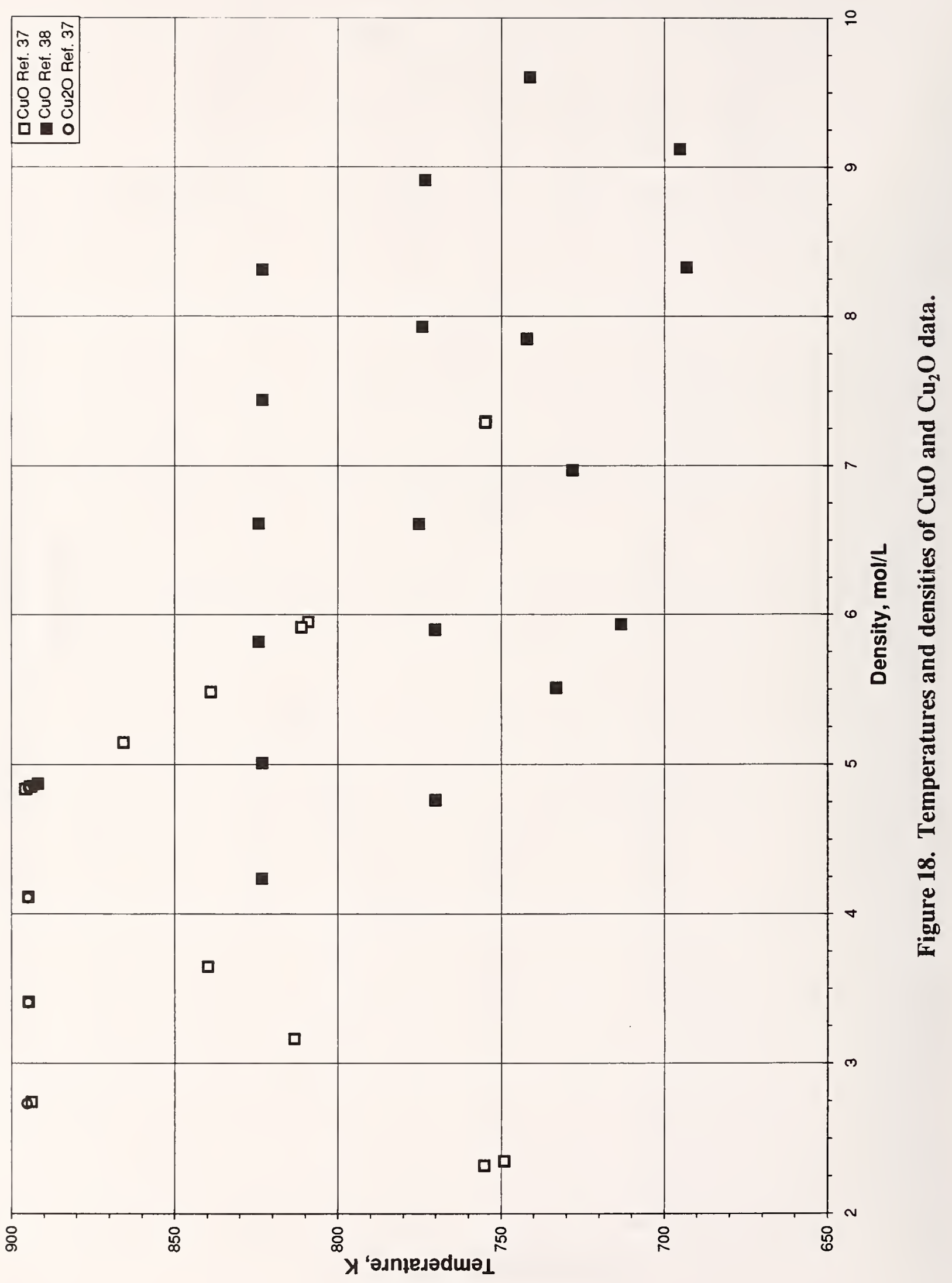




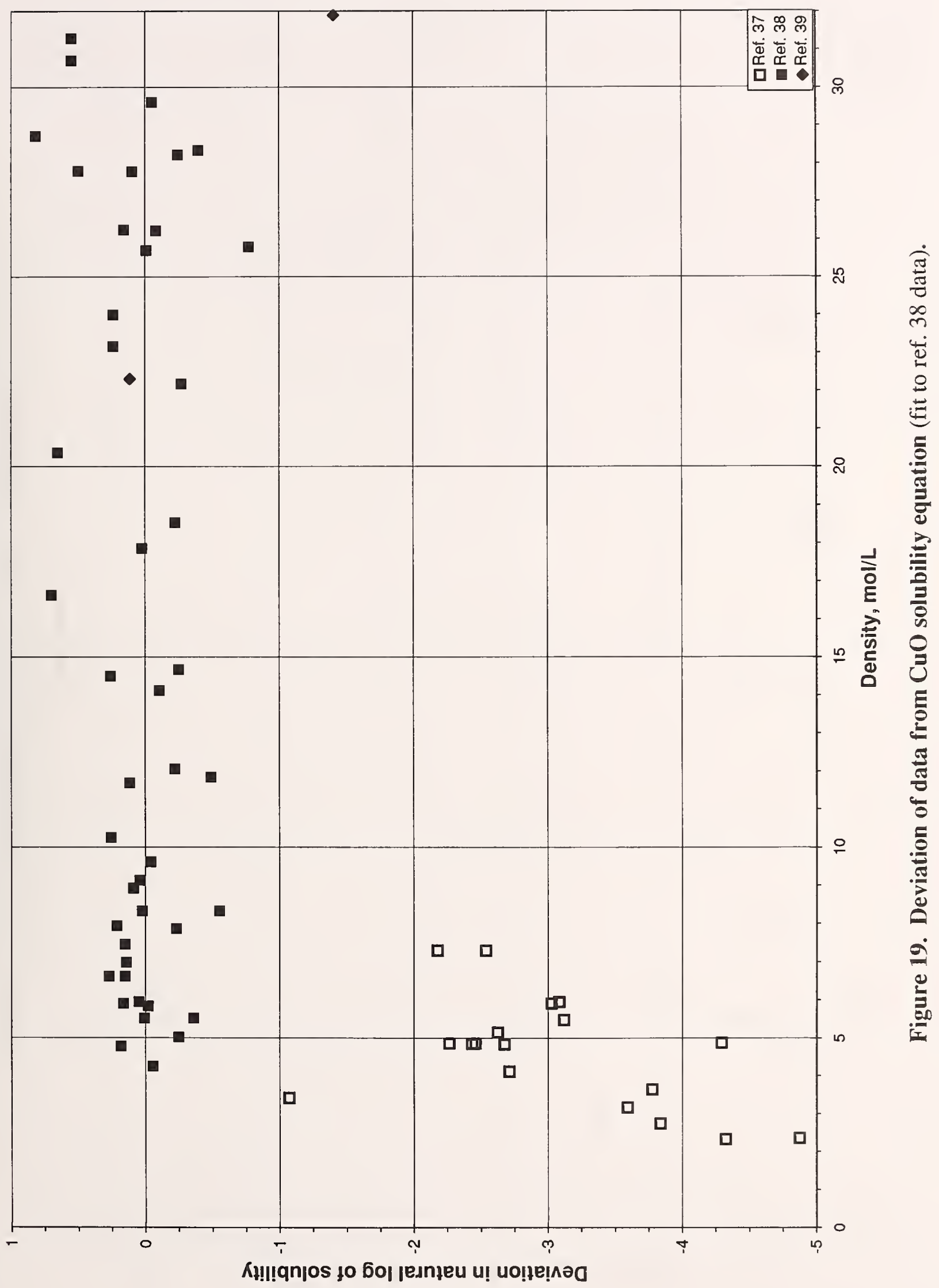




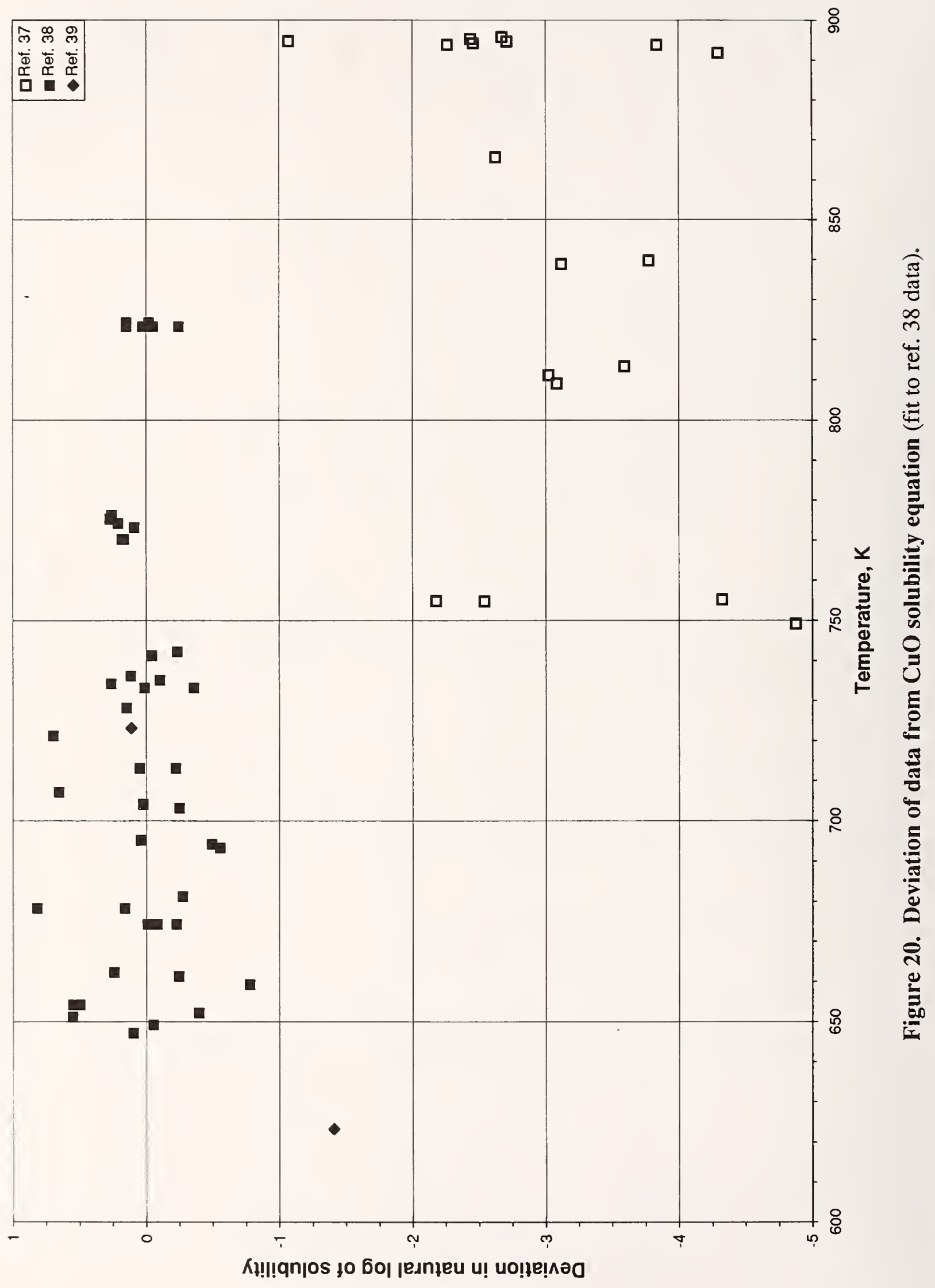




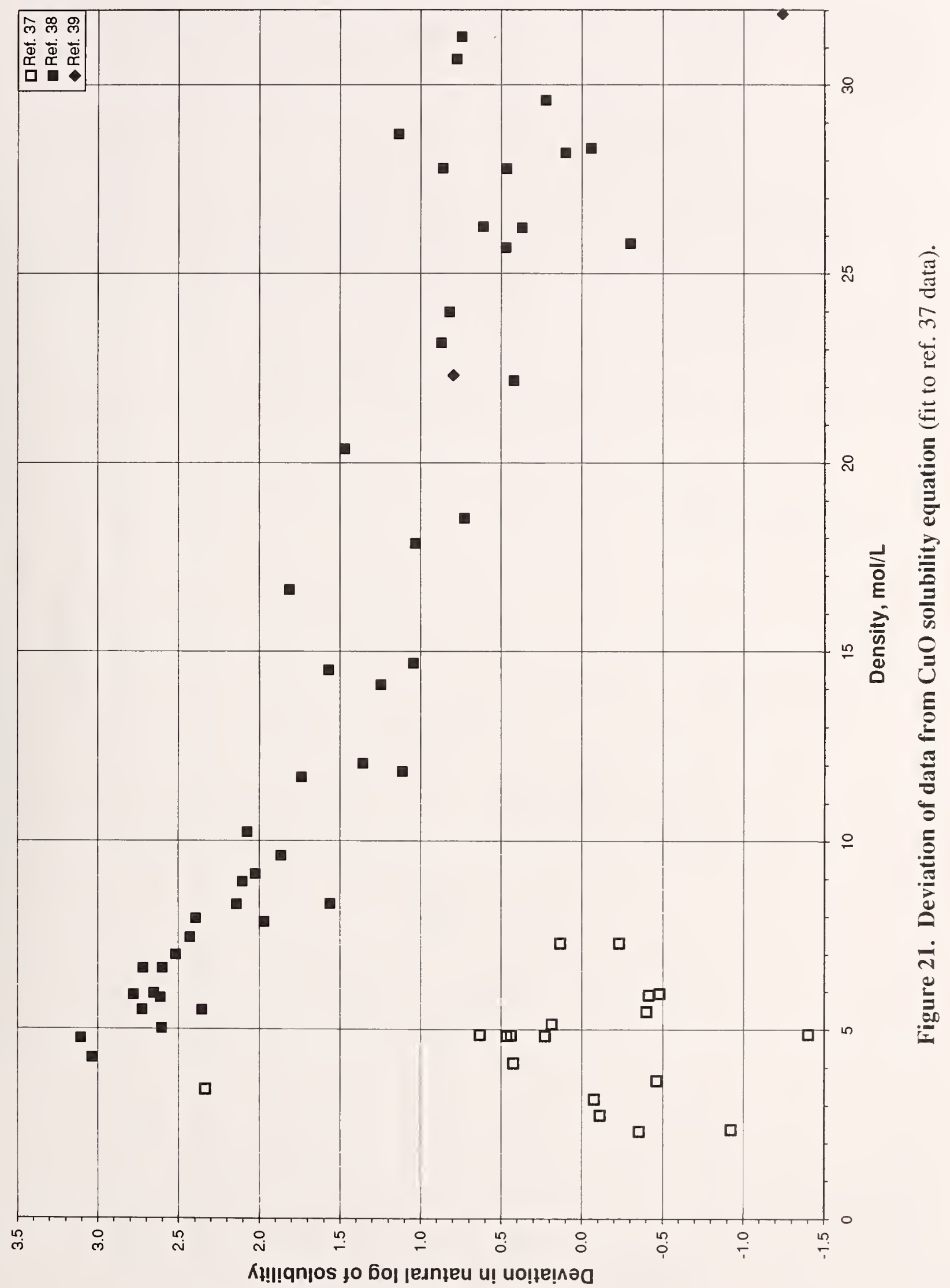




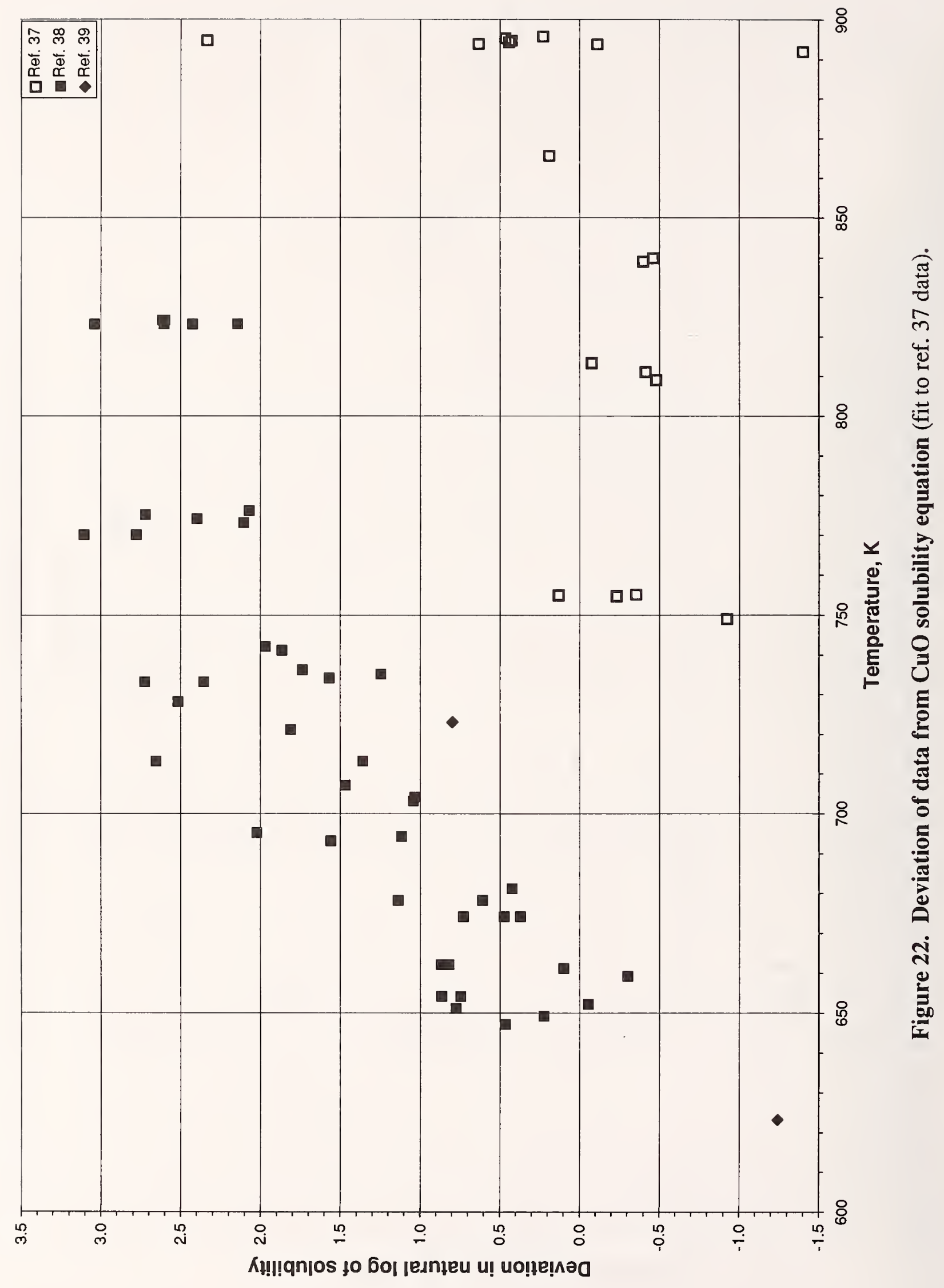




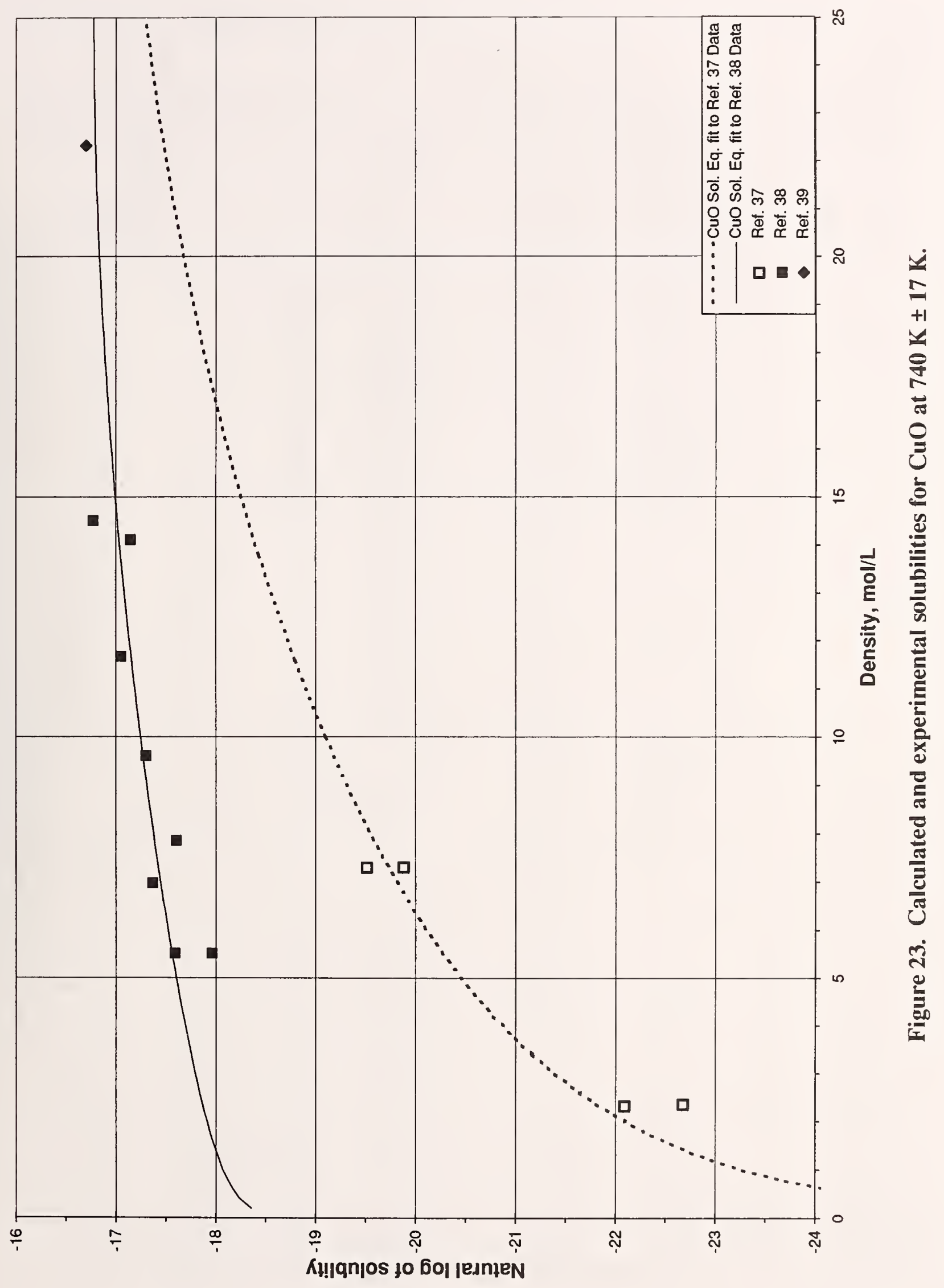




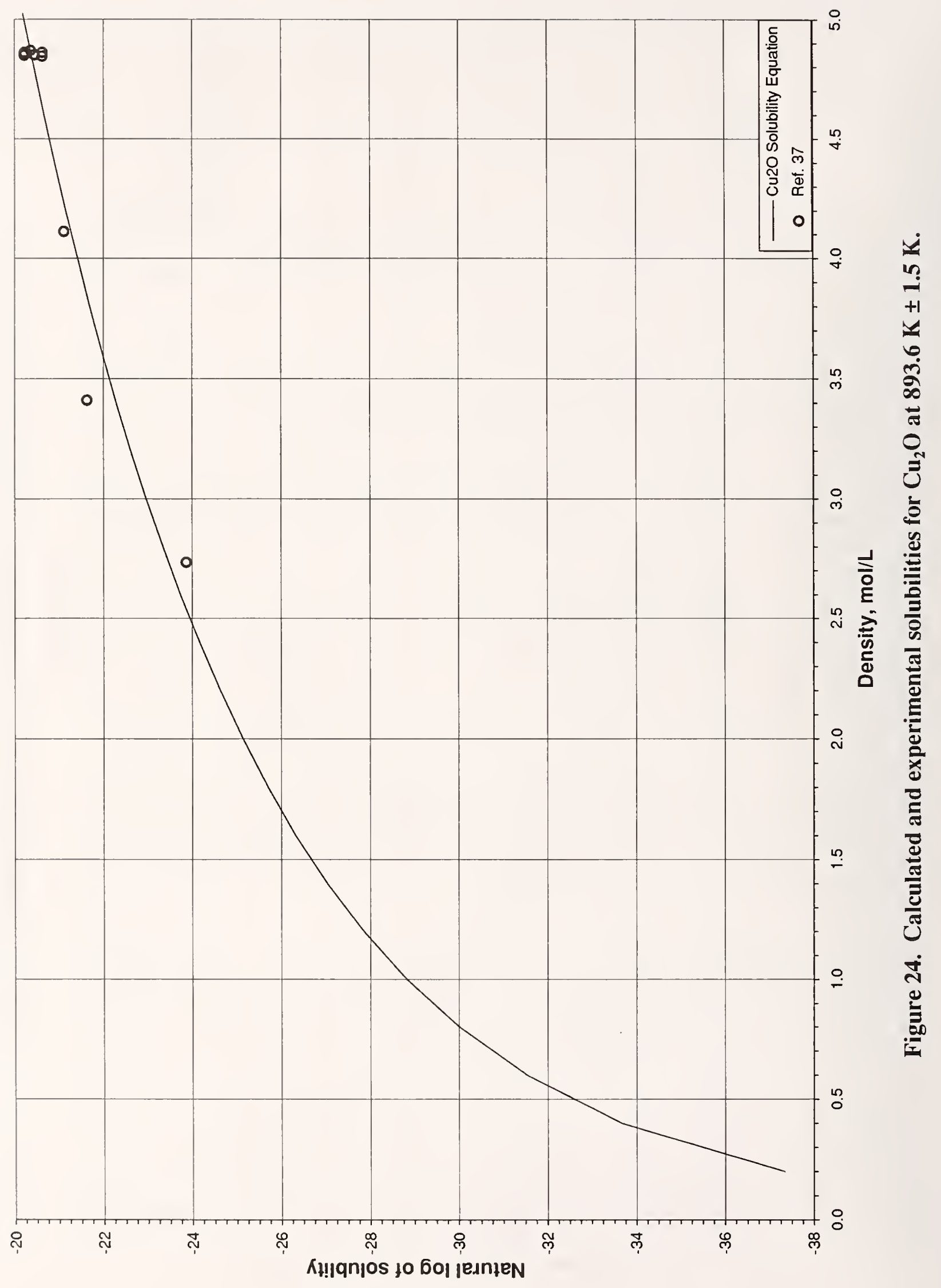


APPENDIX A - SOLUBILITY DATA

\section{Compound: Cupric Oxide ( $\mathrm{CuO})$}

Reference: Pocock, F.J. and Stewart, J.F., 1963, The solubility of copper and its oxides in supercritical 37 steam: Journal of Engineering for Power, v. 85 p. 33-45

Units of original measurements: Temperature in degrees $F$, Pressure in psig, Solubility in ppb of $\mathrm{Cu}$

\begin{tabular}{cccc}
$\begin{array}{c}\text { Temperature } \\
(\mathrm{K})\end{array}$ & $\begin{array}{c}\text { Pressure } \\
\text { (MPa) }\end{array}$ & $\begin{array}{c}\text { Density } \\
\text { (mol/L) }\end{array}$ & $\begin{array}{c}\text { Solubility } \\
\text { (mole fraction) }\end{array}$ \\
\hline 749.04 & 12.8566 & 2.349 & $1.4175 \mathrm{E}-10$ \\
754.76 & 31.1276 & 7.295 & $2.3247 \mathrm{E}-09$ \\
754.93 & 31.1276 & 7.289 & $3.3453 \mathrm{E}-09$ \\
755.26 & 12.8566 & 2.318 & $2.5515 \mathrm{E}-10$ \\
809.09 & 31.1276 & 5.951 & $1.6443 \mathrm{E}-09$ \\
811.09 & 31.1276 & 5.916 & $1.7577 \mathrm{E}-09$ \\
813.37 & 18.7171 & 3.165 & $8.2215 \mathrm{E}-10$ \\
838.93 & 31.1276 & 5.484 & $1.7860 \mathrm{E}-09$ \\
839.82 & 22.1645 & 3.647 & $8.2215 \mathrm{E}-10$ \\
865.59 & 31.1276 & 5.150 & $3.2602 \mathrm{E}-09$ \\
891.87 & 31.1276 & 4.874 & $6.8040 \mathrm{E}-10$ \\
893.82 & 31.1276 & 4.856 & $5.2164 \mathrm{E}-09$ \\
893.87 & 18.7171 & 2.743 & $9.3555 \mathrm{E}-10$ \\
894.26 & 31.1276 & 4.852 & $4.3092 \mathrm{E}-09$ \\
894.71 & 22.8539 & 3.413 & $1.5706 \mathrm{E}-08$ \\
894.71 & 26.9908 & 4.116 & $3.2035 \mathrm{E}-09$ \\
895.32 & 31.1276 & 4.841 & $4.4226 \mathrm{E}-09$ \\
895.82 & 31.1276 & 4.837 & $3.4870 \mathrm{E}-09$ \\
\hline
\end{tabular}

Reference: Hearn, B., Hunt, M. R. and Hayward, A., 1969, Solubility of cupric oxide in pure subcritical and 38 supercritical water: Journal of Chemical and Engineering Data, v. 14, n. 4, p. 442-447

Units of original measurements: Temperature in degrees $C$, Pressure in psig, Solubility in ppb of $\mathrm{Cu}$

Temperature Pressure Density Solubility (K) (MPa) (mol/L) (mole fraction)

330.15 $(\mathrm{MPa})$ 54.945

342.15 8.3750 54.506 3.5154E-09

354.15 7.6855 54.095 4.5360E-09

360.15

361.15

362.15

383.15

390.15

394.15

395.15

406.15

427.15

454.15

460.15

480.15
26.5082

26.6460

27.3355

22.8539

25.6118

24.3708

23.8882

8.3750

8.3750

18.3034

13.9253

13.2013
54.338

54.306

54.286

53.376

53.156

52.954

52.899

51.982

50.941

49.795

49.292

48.024
6.5205E-09

6.6622E-09

6.0952E-09

4.8195E-09

6.6622E-09

8.2215E-09

1.0773E-08

6.5205E-09

7.3710E-09

$1.4742 \mathrm{E}-08$

5.6133E-08

4.0540E-08

$6.0102 \mathrm{E}-08$

Temperature Pressure Density Solubility

(K)

505.15

507.15

522.15

525.15

533.15

533.15

533.15

546.15

564.15

571.15

580.15

595.15

604.15

623.15

623.15

647.15

649.15

651.15

652.15

654.15

654.15

654.15

655.15

659.15

661.15

662.15

662.15

674.15

674.15

674.15

678.15

678.15

681.15

693.15

694.15

695.15

703.15

704.15

707.15

713.15

713.15

721.15

728.15

733.15

733.15

734.15

735.15

736.15
(MPa) $(\mathrm{mol} / \mathrm{L})$ (mole fraction)

20.9579

20.0961

20.9234

26.1634

26.9908

28.6110

22.1645

28.0595

22.3368

29.0592

22.6471

29.0592

27.6803

41.4697

24.2329

27.6803

31.1276

27.3355

27.6803

34.5750

38.0224

41.4697

28.0250

31.8171

27.6458

28.0595

29.7831

34.5750

35.2645

37.1605

41.9524

34.0579

26.5771

30.6795

27.9216

34.9887

38.0224

41.4697

23.9226

34.3682

41.4008

27.7492

24.2329

24.2329

41.8145

41.4697

37.9534
46.675

46.806

45.382

45.196

44.865

44.912

45.002

43.549

42.361

41.210

40.897

38.509

38.208

35.298

37.486

27.786

29.599

30.694

28.323

27.798

31.285

32.326

33.012

25.796

28.209

23.163

23.989

18.539

25.687

26.217

26.234

28.707

22.172

8.329

11.830

9.122

14.672

17.851

20.359

5.937

12.042

16.624

6.970

5.511

5.511

14.501

14.107

11.675
7.9663E-08

8.1931E-08

1.0206E-07

1.1085E-07

1.1142E-07

1.0716E-07

1.1198E-07

1.2587E-07

1.4260E-07

1.4629E-07

1.5507E-07

1.7294E-07

1.7010E-07

$1.3098 \mathrm{E}-07$

1.6160E-07

6.6339E-08

5.4999E-08

9.5539E-08

$3.8839 E-08$

$9.3838 \mathrm{E}-08$

$9.0720 \mathrm{E}-08$

9.9225E-08

1.3778E-07

2.5231E-08

4.2808E-08

6.4354E-08

6.6055E-08

3.3169E-08

5.1597E-08

4.8195E-08

6.0669E-08

$1.1510 \mathrm{E}-07$

3.6571E-08

1.3041E-08

1.8144E-08

2.5515E-08

2.8350 E- 08

4.3375E-08

8.9302E-08

2.1829E-08

2.6082E-08

$8.4766 \mathrm{E}-08$

2.8633E-08

$1.5876 \mathrm{E}-08$

2.2963E-08

5.2164E-08

3.5721E-08

3.9406E-08 


\begin{tabular}{ccrc}
$\begin{array}{c}\text { Temperature } \\
(\mathrm{K})\end{array}$ & $\begin{array}{c}\text { Pressure } \\
\text { (MPa) }\end{array}$ & $\begin{array}{c}\text { Density } \\
\text { (mol/L) }\end{array}$ & $\begin{array}{c}\text { Solubility } \\
\text { (mole fraction) }\end{array}$ \\
\hline 741.15 & 34.9887 & 9.603 & $3.0618 \mathrm{E}-08$ \\
742.15 & 31.2655 & 7.848 & $2.2680 \mathrm{E}-08$ \\
770.15 & 23.9916 & 4.764 & $3.2035 \mathrm{E}-08$ \\
770.15 & 28.1284 & 5.901 & $3.4303 \mathrm{E}-08$ \\
773.15 & 37.5052 & 8.910 & $3.9123 \mathrm{E}-08$ \\
774.15 & 34.8852 & 7.931 & $4.1958 \mathrm{E}-08$ \\
775.15 & 30.8863 & 6.606 & $4.1107 \mathrm{E}-08$ \\
776.15 & 41.3318 & 10.234 & $5.0463 \mathrm{E}-08$ \\
823.15 & 24.4397 & 4.241 & $3.2602 \mathrm{E}-08$ \\
823.15 & 28.0250 & 5.011 & $2.8350 \mathrm{E}-08$ \\
823.15 & 38.0913 & 7.439 & $4.8762 \mathrm{E}-08$ \\
823.15 & 41.3318 & 8.311 & $4.5076 \mathrm{E}-08$ \\
824.15 & 31.6447 & 5.821 & $3.7705 \mathrm{E}-08$ \\
824.15 & 34.9197 & 6.609 & $4.6777 \mathrm{E}-08$ \\
\hline
\end{tabular}

Reference: Var'yash, L.N., 1986, Hydrolysis of Cu(II) at 25350 degrees $\mathrm{C}$ : Geochemistry International, v.

\section{Compound: Cuprous Oxide ( $\mathrm{Cu2O})$}

Reference: Pocock, F.J. and Stewart, J.F., 1963, The 37 solubility of copper and its oxides in supercritical steam: Journal of Engineering for Power, v. 85. p. $33-45$

Units of original measurements: Temperature in degrees $F$, Pressure in psig, Solubility in ppb of $\mathrm{Cu}$

\begin{tabular}{cccc}
$\begin{array}{c}\text { Temperature } \\
(\mathrm{K})\end{array}$ & $\begin{array}{c}\text { Pressure } \\
(\mathrm{MPa})\end{array}$ & $\begin{array}{c}\text { Density } \\
(\mathrm{mol} / \mathrm{L})\end{array}$ & $\begin{array}{c}\text { Solubility } \\
\text { (mole fraction) }\end{array}$ \\
\hline 892.21 & 31.1276 & 4.871 & $1.4033 \mathrm{E}-09$ \\
892.93 & 31.1276 & 4.864 & $1.0915 \mathrm{E}-09$ \\
893.04 & 31.1276 & 4.863 & $1.6301 \mathrm{E}-09$ \\
894.09 & 31.1276 & 4.853 & $1.2899 \mathrm{E}-09$ \\
894.21 & 31.1276 & 4.852 & $1.6301 \mathrm{E}-09$ \\
894.59 & 31.1276 & 4.848 & $1.0915 \mathrm{E}-09$ \\
894.71 & 22.8539 & 3.413 & $3.9690 \mathrm{E}-10$ \\
894.71 & 26.9908 & 4.116 & $6.6622 \mathrm{E}-10$ \\
895.04 & 18.7171 & 2.738 & $4.2525 \mathrm{E}-11$ \\
\hline
\end{tabular}

Units of original measurements: Temperature in degrees $C$

Solubility in molality, "indicates data point is at saturation

Temperature Pressure Density Solubility

\begin{tabular}{clcc}
$(\mathrm{K})$ & $(\mathrm{MPa})$ & $(\mathrm{mol} / \mathrm{L})$ & $($ mole fraction $)$ \\
\hline 623.15 & 16.5294 & 31.901 & $1.8916 \mathrm{E}-08^{*}$ \\
723.15 & 50.0000 & 22.317 & $5.5667 \mathrm{E}-08$ \\
\hline
\end{tabular}


Compound: Quartz (SiO2)

$\begin{array}{cl}\text { Reference: } & \text { Heitmann, H.G., 1964, Solubility of silicic acid in } \\ \text { water and steam and their influence on turbine } \\ \text { 19 } & \text { silication: Chemiker-Ztg./Chem. Apparatur, v. } 88, \\ & \text { n. } 22, \text { p. } 891-893\end{array}$

Units of original measurements: Temperature in degrees $C$,

Pressure in $\mathrm{kg} / \mathrm{cm} 2$, Solubility in $\mathrm{mg} / \mathrm{kg}$

Temperature Pressure Density Solubility

\begin{tabular}{rrrr}
\multicolumn{1}{c}{$(\mathrm{K})$} & \multicolumn{1}{c}{$(\mathrm{MPa})$} & \multicolumn{1}{c}{ (mol/L) } & (mole fraction) \\
\hline 425.15 & 0.0981 & 0.028 & $6.8962 \mathrm{E}-09$ \\
433.15 & 29.4200 & 51.271 & $3.6283 \mathrm{E}-05$ \\
435.15 & 0.0981 & 0.027 & $7.7957 \mathrm{E}-09$ \\
440.15 & 0.2942 & 0.082 & $1.3792 \mathrm{E}-08$ \\
473.15 & 0.2942 & 0.076 & $1.5891 \mathrm{E}-08$ \\
478.15 & 29.4200 & 48.784 & $7.7671 \mathrm{E}-05$ \\
488.15 & 0.0981 & 0.024 & $1.0194 \mathrm{E}-08$ \\
508.15 & 0.2942 & 0.070 & $2.2787 \mathrm{E}-08$ \\
513.15 & 29.4200 & 46.511 & $1.2627 \mathrm{E}-04$ \\
525.15 & 0.5884 & 0.138 & $4.1377 \mathrm{E}-08$ \\
525.15 & 3.1381 & 0.823 & $5.9967 \mathrm{E}-08$ \\
543.15 & 0.0981 & 0.022 & $1.6191 \mathrm{E}-08$ \\
548.15 & 0.2942 & 0.065 & $2.8184 \mathrm{E}-08$ \\
553.15 & 29.4200 & 43.430 & $1.9288 \mathrm{E}-04$ \\
573.15 & 4.9033 & 1.196 & $3.7779 \mathrm{E}-07$ \\
573.15 & 29.4200 & 41.620 & $2.2619 \mathrm{E}-04$ \\
577.15 & 3.1381 & 0.711 & $1.4992 \mathrm{E}-07$ \\
583.15 & 0.2942 & 0.061 & $4.1077 \mathrm{E}-08$ \\
588.15 & 0.5884 & 0.122 & $7.7357 \mathrm{E}-08$ \\
589.15 & 0.0981 & 0.020 & $2.6086 \mathrm{E}-08$ \\
603.15 & 29.4200 & 38.378 & $2.7542 \mathrm{E}-04$ \\
613.15 & 0.0981 & 0.019 & $4.1077 \mathrm{E}-08$ \\
623.15 & 0.2942 & 0.057 & $6.3865 \mathrm{E}-08$ \\
623.15 & 4.9033 & 1.045 & $6.5364 \mathrm{E}-07$ \\
626.15 & 9.8066 & 2.381 & $2.0839 \mathrm{E}-06$ \\
627.15 & 3.1381 & 0.638 & $3.8079 \mathrm{E}-07$ \\
633.15 & 29.4200 & 33.964 & $2.8743 \mathrm{E}-04$ \\
655.15 & 0.0981 & 0.018 & $5.3370 \mathrm{E}-08$ \\
658.15 & 0.5884 & 0.108 & $1.5172 \mathrm{E}-07$ \\
663.15 & 0.2942 & 0.054 & $1.1484 \mathrm{E}-07$ \\
663.15 & 29.4200 & 25.381 & $1.8388 \mathrm{E}-04$ \\
673.15 & 4.9033 & 0.940 & $1.1634 \mathrm{E}-06$ \\
673.15 & 9.8066 & 2.051 & $3.1483 \mathrm{E}-06$ \\
673.15 & 19.6133 & 5.386 & $1.3793 \mathrm{E}-05$ \\
674.15 & 3.1381 & 0.584 & $6.7163 \mathrm{E}-07$ \\
681.15 & 29.4200 & 13.678 & $9.8368 \mathrm{E}-05$ \\
683.15 & 0.5884 & 0.104 & $1.9789 \mathrm{E}-07$ \\
703.15 & 0.0981 & 0.017 & $9.6846 \mathrm{E}-08$ \\
711.15 & 0.2942 & 0.050 & $1.5052 \mathrm{E}-07$ \\
717.15 & 29.4200 & 8.303 & $4.6479 \mathrm{E}-05$ \\
722.15 & 19.6133 & 4.264 & $1.6791 \mathrm{E}-05$ \\
723.15 & 4.9033 & 0.859 & $2.8424 \mathrm{E}-06$ \\
723.15 & 9.8066 & 1.823 & $5.3071 \mathrm{E}-06$ \\
768.15 & 29.4200 & 6.333 & $5.0978 \mathrm{E}-05$
\end{tabular}

Temperature Pressure Density Solubility

\begin{tabular}{crrr}
$(\mathrm{K})$ & \multicolumn{1}{c}{$(\mathrm{MPa})$} & $(\mathrm{mol} / \mathrm{L})$ & $($ mole fraction $)$ \\
\hline 773.15 & 4.9033 & 0.793 & $3.6580 \mathrm{E}-06$ \\
773.15 & 9.8066 & 1.656 & $9.5649 \mathrm{E}-06$ \\
773.15 & 19.6133 & 3.663 & $2.2789 \mathrm{E}-05$ \\
820.15 & 29.4200 & 5.366 & $6.3574 \mathrm{E}-05$ \\
823.15 & 3.1381 & 0.467 & $3.4181 \mathrm{E}-06$ \\
823.15 & 9.8066 & 1.525 & $1.4632 \mathrm{E}-05$ \\
823.15 & 19.6133 & 3.275 & $3.4484 \mathrm{E}-05$ \\
827.15 & 4.9033 & 0.734 & $3.7479 \mathrm{E}-06$ \\
862.15 & 19.6133 & 3.044 & $4.0181 \mathrm{E}-05$ \\
863.15 & 29.4200 & 4.841 & $7.7371 \mathrm{E}-05$ \\
865.15 & 4.9033 & 0.698 & $8.0057 \mathrm{E}-06$ \\
865.15 & 9.8066 & 1.433 & $1.9100 \mathrm{E}-05$ \\
\hline
\end{tabular}

Reference: Wendlandt. H.G., 1963, Reaktionen zwischen Oxiden und Wasser bei hoheren Temperaturen 20 und verschiednen Dichten: Dissertation University of Gottingen

Units of original measurements: Temperature in degrees $C$, Pressure in atm, Solubility in $\mathrm{mg} / \mathrm{kg}$

Temperature Pressure Density Solubility (K) (MPa) (mol/L) (mole fraction)

$\begin{array}{rrrr}673.15 & 2.1278 & 0.391 & 2.0988 \mathrm{E}-07 \\ 673.15 & 4.5596 & 0.869 & 4.4975 \mathrm{E}-07 \\ 673.15 & 6.5861 & 1.298 & 6.8962 \mathrm{E}-07 \\ 673.15 & 9.3219 & 1.931 & 9.5947 \mathrm{E}-07 \\ 673.15 & 10.6391 & 2.264 & 1.4092 \mathrm{E}-06 \\ 673.15 & 15.1988 & 3.609 & 2.8484 \mathrm{E}-06 \\ 673.15 & 18.2385 & 4.759 & 3.8379 \mathrm{E}-06 \\ 673.15 & 20.2650 & 5.715 & 5.5770 \mathrm{E}-06 \\ 673.15 & 22.2915 & 6.917 & 1.1094 \mathrm{E}-05 \\ 673.15 & 25.3313 & 9.627 & 1.9490 \mathrm{E}-05 \\ 673.15 & 27.8644 & 14.061 & 3.8082 \mathrm{E}-05 \\ 673.15 & 28.8776 & 16.805 & 5.7276 \mathrm{E}-05 \\ 673.15 & 29.8909 & 19.577 & 1.0257 \mathrm{E}-04 \\ 673.15 & 35.4637 & 26.688 & 1.8658 \mathrm{E}-04 \\ 673.15 & 40.5300 & 29.264 & 2.4630 \mathrm{E}-04 \\ 773.15 & 1.0133 & 0.159 & 1.7990 \mathrm{E}-07 \\ 773.15 & 2.0265 & 0.320 & 2.1588 \mathrm{E}-07 \\ 773.15 & 3.0398 & 0.484 & 2.9983 \mathrm{E}-07 \\ 773.15 & 5.0663 & 0.821 & 6.8962 \mathrm{E}-07 \\ 773.15 & 8.1060 & 1.348 & 1.3193 \mathrm{E}-06 \\ 773.15 & 8.1060 & 1.348 & 1.0494 \mathrm{E}-06 \\ 773.15 & 10.1325 & 1.716 & 8.3954 \mathrm{E}-07 \\ 773.15 & 12.3616 & 2.139 & 2.1288 \mathrm{E}-06 \\ 773.15 & 12.6656 & 2.198 & 2.0389 \mathrm{E}-06 \\ 773.15 & 15.1988 & 2.706 & 3.6580 \mathrm{E}-06 \\ 773.15 & 17.7319 & 3.243 & 5.4570 \mathrm{E}-06 \\ 773.15 & 18.2385 & 3.355 & 6.5964 \mathrm{E}-06 \\ 773.15 & 21.2783 & 4.052 & 2.2789 \mathrm{E}-05 \\ 773.15 & 21.7849 & 4.173 & 2.8186 \mathrm{E}-05 \\ 773.15 & 22.2915 & 4.296 & 1.4992 \mathrm{E}-05 \\ 773.15 & 25.8379 & 5.204 & 2.9386 \mathrm{E}-05\end{array}$




\begin{tabular}{ccrc}
$\begin{array}{c}\text { Temperature } \\
\text { (K) }\end{array}$ & $\begin{array}{c}\text { Pressure } \\
\text { (MPa) }\end{array}$ & $\begin{array}{c}\text { Density } \\
\text { (mol/L) }\end{array}$ & $\begin{array}{c}\text { Solubility } \\
\text { (mole fraction) }\end{array}$ \\
\hline 773.15 & 29.8909 & 6.355 & $3.6283 \mathrm{E}-05$ \\
773.15 & 31.2081 & 6.758 & $5.2177 \mathrm{E}-05$ \\
773.15 & 40.0234 & 9.881 & $5.4877 \mathrm{E}-05$ \\
773.15 & 40.5300 & 10.084 & $8.5770 \mathrm{E}-05$ \\
773.15 & 50.6625 & 14.579 & $1.5177 \mathrm{E}-04$ \\
\hline
\end{tabular}

Reference: Morey, G.W. and Hesselgesser, J.M., 1950, The solubility of quartz and some other substances in 21 superheated steam at high pressures: Joint Research Committee on Boiler Feedwater Studies and the Power Division Annual Meeting, ASME Paper No. 50--A-73, p. 865-875

Units of original measurements: Temperature in degrees $C$, Pressure in psia, Solubility in $p p m$

\begin{tabular}{crrr}
$\begin{array}{c}\text { Temperature } \\
(\mathrm{K})\end{array}$ & \multicolumn{1}{c}{$\begin{array}{c}\text { Pressure } \\
\text { (MPa) }\end{array}$} & $\begin{array}{c}\text { Density } \\
\text { (mol/L) }\end{array}$ & $\begin{array}{c}\text { Solubility } \\
\text { (mole fraction) }\end{array}$ \\
\hline 633.15 & 34.4737 & 35.053 & $2.5561 \mathrm{E}-04$ \\
673.15 & 3.4474 & 0.646 & $3.1183 \mathrm{E}-07$ \\
673.15 & 6.8947 & 1.366 & $9.2949 \mathrm{E}-07$ \\
673.15 & 13.7895 & 3.154 & $1.5591 \mathrm{E}-06$ \\
673.15 & 34.4737 & 25.969 & $1.9108 \mathrm{E}-04$ \\
673.15 & 68.9473 & 35.301 & $3.7782 \mathrm{E}-04$ \\
673.15 & 103.4210 & 38.737 & $4.5052 \mathrm{E}-04$ \\
773.15 & 3.4474 & 0.551 & $1.1304 \mathrm{E}-06$ \\
773.15 & 6.8947 & 1.134 & $4.2577 \mathrm{E}-06$ \\
773.15 & 13.7895 & 2.420 & $1.0704 \mathrm{E}-05$ \\
773.15 & 34.4737 & 7.825 & $6.4774 \mathrm{E}-05$ \\
773.15 & 68.9473 & 22.188 & $4.0456 \mathrm{E}-04$ \\
773.15 & 103.4210 & 29.853 & $7.7978 \mathrm{E}-04$ \\
\hline
\end{tabular}

Reference: Morey, G.W. and Hesselgesser, J.M., 1951, The solubility of some minerals in superheated steam 22 at high pressures: Economic Geology, v. 46, p. 821-835

Units of original measurements: Temperature in degrees $C$, Pressure in psia, Solubility in wt\%

\begin{tabular}{crrr}
$\begin{array}{c}\text { Temperature } \\
(\mathrm{K})\end{array}$ & \multicolumn{1}{c}{$\begin{array}{c}\text { Pressure } \\
(\mathrm{MPa})\end{array}$} & $\begin{array}{c}\text { Density } \\
\text { (mol/L) }\end{array}$ & $\begin{array}{c}\text { Solubility } \\
\text { (mole fraction) }\end{array}$ \\
\hline 673.15 & 150.0000 & 41.701 & $6.2156 \mathrm{E}-04$ \\
673.15 & 200.0000 & 43.992 & $6.9975 \mathrm{E}-04$ \\
773.15 & 150.0000 & 34.971 & $1.2148 \mathrm{E}-03$ \\
773.15 & 200.0000 & 38.380 & $1.5014 \mathrm{E}-03$ \\
873.15 & 33.3000 & 5.490 & $1.0797 \mathrm{E}-04$ \\
873.15 & 100.0000 & 20.772 & $8.8935 \mathrm{E}-04$ \\
873.15 & 150.0000 & 28.192 & $1.6827 \mathrm{E}-03$ \\
873.15 & 200.0000 & 32.714 & $2.3061 \mathrm{E}-03$ \\
\hline
\end{tabular}

Reference: Kennedy, G.C., 1950, A portion of the system 23653

Units of original measurements: Temperature in degrees $C$, Pressure in bar, Solubility in wt\%

Temperature Pressure Density Solubility

\begin{tabular}{cccc}
$(\mathrm{K})$ & $(\mathrm{MPa})$ & $(\mathrm{mol} / \mathrm{L})$ & $($ mole fraction $)$ \\
\hline 433.15 & 0.6200 & 50.371 & $2.0989 \mathrm{E}-05$
\end{tabular}

Temperature Pressure Density Solubility (K)

455.15

473.15

495.15

513.15

529.15

536.15

553.15

553.15

573.15

573.15

573.15

590.15

590.15

591.15

593.15

598.15

598.15

599.15

603.15

603.15

606.15

609.15

610.15

612.15

614.15

616.15

619.15

621.15

623.15

623.15

628.15

628.15

631.15

633.15

633.15

633.15

633.15

633.15

634.15

638.15

639.15

641.15

641.15

641.15

642.15

642.15

645.15

646.15

647.15

648.15

651.15
(MPa) (mol/L) (mole fraction)

1.1100

1.5500 49.117

47.996

5.0978E-05

2.5000

46.497

7.1972E-05

$100.0000 \quad 48.325 \quad 1.8898 \mathrm{E}-04$

$5.1700 \quad 43.234 \quad 1.7097 \mathrm{E}-04$

$6.4200 \quad 41.647 \quad 1.8898 \mathrm{E}-04$

$100.0000 \quad 46.926 \quad 2.3400$ E- 04

$8.5900 \quad 39.530 \quad 2.0398 \mathrm{E}-04$

$30.0000 \quad 41.668 \quad 2.3100 \mathrm{E}-04$

$30.0000 \quad 41.668 \quad 2.3100 \mathrm{E}-04$

$50.0000 \quad 41.664 \quad 2.6101 \mathrm{E}-04$

$100.0000 \quad 44.594 \quad 3.1506 \mathrm{E}-04$

$10.6700 \quad 37.302 \quad 2.1299 \mathrm{E}-04$

$20.0000 \quad 38.415 \quad 2.4000 \mathrm{E}-04$

$15.0000 \quad 36.906 \quad 2.3100 \mathrm{E}-04$

$30.0000 \quad 39.043 \quad 2.6402 \mathrm{E}-04$

$25.0000 \quad 38.308 \quad 2.4600 \mathrm{E}-04$

$12.8100 \quad 35.568 \quad 2.2199 \mathrm{E}-04$

$75.0000 \quad 42.299 \quad 3.1205 \mathrm{E}-04$

$100.0000 \quad 43.514 \quad 3.5710 \mathrm{E}-04$

$25.0000 \quad 36.956 \quad 2.5801 \mathrm{E}-04$

$20.0000 \quad 35.895 \quad 2.4000 \mathrm{E}-04$

$15.0000 \quad 34.262 \quad 2.2799 \mathrm{E}-04$

$30.0000 \quad 37.038 \quad 2.7302 \mathrm{E}-04$

$50.0000 \quad 39.208 \quad 3.1506 \mathrm{E}-04$

$15.6500 \quad 32.751 \quad 2.0999 E-04$

$35.0000 \quad 36.848 \quad 2.9104 \mathrm{E}-04$

$20.0000 \quad 33.340 \quad 2.4000$ E- 04

$100.0000 \quad 42.316 \quad 4.0516 \mathrm{E}-04$

$30.0000 \quad 34.956 \quad 2.9104 \mathrm{E}-04$

$75.0000 \quad 40.259 \quad 3.6911 \mathrm{E}-04$

$25.0000 \quad 33.153 \quad 2.6101 \mathrm{E}-04$

$18.6700 \quad 29.286 \quad 1.7097 \mathrm{E}-04$

$18.6700 \quad 29.286 \quad 1.8298 \mathrm{E}-04$

$18.6700 \quad 29.286 \quad 1.6797 \mathrm{E}-04$

$20.0000 \quad 30.419 \quad 2.0999 E-04$

$30.0000 \quad 34.104 \quad 2.9104 \mathrm{E}-04$

$40.0000 \quad 35.875 \quad 3.2106 \mathrm{E}-04$

$20.0000 \quad 27.832 \quad 1.9048 \mathrm{E}-04$

$35.0000 \quad 34.208 \quad 3.1205 \mathrm{E}-04$

$20.0000 \quad 8.513 \quad 1.3947 \mathrm{E}-04$

$20.5100 \quad 26.182 \quad 1.3947 \mathrm{E}-04$

$50.0000 \quad 36.453 \quad 3.5410 \mathrm{E}-04$

$25.0000 \quad 30.325 \quad 2.2799 \mathrm{E}-04$

$30.0000 \quad 32.360 \quad 2.7002 E-04$

$30.0000 \quad 31.698 \quad 2.6702 \mathrm{E}-04$

$22.1000 \quad 22.130 \quad 6.8973 \mathrm{E}-05$

$25.0000 \quad 28.501 \quad 1.7697 \mathrm{E}-04$

$25.0000 \quad 28.059 \quad 1.8298 \mathrm{E}-04$

$75.0000 \quad 38.203$

4.3220E-04 


\begin{tabular}{|c|c|c|c|}
\hline $\begin{array}{c}\text { Temperature } \\
(\mathrm{K})\end{array}$ & $\begin{array}{c}\text { Pressure } \\
(\mathrm{MPa})\end{array}$ & $\begin{array}{l}\text { Density } \\
(\mathrm{mol} / \mathrm{L})\end{array}$ & $\begin{array}{c}\text { Solubility } \\
\text { (mole fraction) }\end{array}$ \\
\hline 653.15 & 25.0000 & 25.024 & 1.3797E-04 \\
\hline 653.15 & 50.0000 & 34.946 & $3.5410 E-04$ \\
\hline 654.15 & 30.0000 & 29.340 & 2.3400E-04 \\
\hline 655.15 & 100.0000 & 39.910 & $4.8328 \mathrm{E}-04$ \\
\hline 658.15 & 30.0000 & 28.031 & 2.1899E-04 \\
\hline 658.15 & 35.0000 & 30.544 & 2.8503E-04 \\
\hline 662.15 & 25.0000 & 12.500 & 4.7979E-05 \\
\hline 663.15 & 30.0000 & 26.023 & $1.9048 \mathrm{E}-04$ \\
\hline 664.15 & 30.0000 & 25.552 & $1.8298 E-04$ \\
\hline 665.15 & 35.0000 & 28.792 & $2.4600 \mathrm{E}-04$ \\
\hline 665.15 & 40.0000 & 30.784 & 3.0605E-04 \\
\hline 667.15 & 125.0000 & 40.679 & 5.6744E-04 \\
\hline 668.15 & 50.0000 & 32.840 & $3.5710 \mathrm{E}-04$ \\
\hline 669.15 & 50.0000 & 32.689 & $3.5410 E-04$ \\
\hline 670.15 & 30.0000 & 22.018 & 1.4397E-04 \\
\hline 673.15 & 35.0000 & 26.365 & $2.2799 \mathrm{E}-04$ \\
\hline 674.15 & 60.0000 & 33.868 & 4.1417E-04 \\
\hline 680.15 & 40. & 27.323 & 3E-04 \\
\hline 681.15 & 30. & 14.783 & $9 E-05$ \\
\hline 683.15 & 60.0000 & 32.689 & $4.0816 \mathrm{E}-04$ \\
\hline 683.15 & 75.0000 & 35.003 & 4.6224E-04 \\
\hline 684.15 & 100.0000 & 37.546 & 5.4339E-04 \\
\hline 686.15 & 175.0000 & 42.162 & 7.6594E-04 \\
\hline 689.15 & 100.0000 & 37.121 & $5.5842 \mathrm{E}-04$ \\
\hline 693.15 & 000 & 18.000 & 1.4547E-04 \\
\hline 696.15 & 50. & 28.021 & $7 E-04$ \\
\hline 700.15 & 150. & 39.952 & 1 E-04 \\
\hline 701.15 & 000 & 15.236 & 1.1847E-04 \\
\hline 702.15 & 150.0000 & 39.820 & $8.1409 E-04$ \\
\hline 703.15 & 40.0000 & 20.231 & $1.9648 \mathrm{E}-04$ \\
\hline 708.15 & 175.0000 & 40.848 & $9.1946 \mathrm{E}-04$ \\
\hline 709.15 & 75.0000 & 32.076 & 4.8027E-04 \\
\hline 711.15 & 50. & 24.928 & 2.9704E-04 \\
\hline 715.15 & 60.0000 & 27.927 & 5E-04 \\
\hline 715.15 & 150.0000 & 38.954 & 8.3215E-04 \\
\hline 722.15 & 75.0000 & 30.504 & 4.7727E-04 \\
\hline 725.15 & 100.0000 & 33.906 & $6.3659 \mathrm{E}-04$ \\
\hline 726.15 & 40.0000 & 14.485 & 1.3797E-04 \\
\hline 728.15 & 50.0000 & 21.247 & $2.7753 \mathrm{E}-04$ \\
\hline 748.15 & 50.0 & 17.497 & $2.4150 \mathrm{E}-04$ \\
\hline 751.15 & 60.0000 & 21.990 & 3.4509E-04 \\
\hline 765.15 & 100.0000 & 30.097 & 7.0276E-04 \\
\hline 777.15 & 75.0000 & 23.562 & 4.9079E-04 \\
\hline 779.15 & 50.0000 & 13.695 & $1.9948 \mathrm{E}-04$ \\
\hline 779.15 & 60.0000 & 18.053 & $3.1806 \mathrm{E}-04$ \\
\hline 780.15 & 60.0000 & 17.934 & 3.2106E-04 \\
\hline 784.15 & 50.0000 & 13.261 & $1.9348 \mathrm{E}-04$ \\
\hline 813.15 & 50.0000 & 11.341 & 1.7697E-04 \\
\hline 815.15 & 100.0000 & 25.383 & $7.5090 \mathrm{E}-04$ \\
\hline 818.15 & 60.0000 & 14.382 & $3.0004 E-04$ \\
\hline 826.15 & 75.0000 & 18.453 & $4.9830 \mathrm{E}-04$ \\
\hline
\end{tabular}

\begin{tabular}{cccc}
$\begin{array}{c}\text { Temperature } \\
(\mathrm{K})\end{array}$ & $\begin{array}{c}\text { Pressure } \\
(\mathrm{MPa})\end{array}$ & $\begin{array}{c}\text { Density } \\
(\mathrm{mol} / \mathrm{L})\end{array}$ & $\begin{array}{c}\text { Solubility } \\
\text { (mole fraction) }\end{array}$ \\
\hline 832.15 & 75.0000 & 17.954 & $5.0131 \mathrm{E}-04$ \\
878.15 & 60.0000 & 11.297 & $2.9404 \mathrm{E}-04$ \\
883.15 & 125.0000 & 24.348 & $1.3716 \mathrm{E}-03$ \\
\hline
\end{tabular}

Reference: Wyart, J. and Sabatier, G., 1955, Nouvelles

$50 \quad$ mesures de la solubilitie du quartz, de la pression au-dessus de la temperature critique Acad. Sci. Paris Comptes Rendus, v. 240, p. 1905-1907

Units of original measurements: Temperature in degrees $C$, Pressure in bar, Solubility in $\mathrm{g} / \mathrm{kg}$

Temperature Pressure Density Solubility

\begin{tabular}{crrr}
$(\mathrm{K})$ & \multicolumn{1}{c}{$(\mathrm{MPa})$} & $(\mathrm{mol} / \mathrm{L})$ & (mole fraction) \\
\hline 673.15 & 50.0000 & 32.072 & $3.3007 \mathrm{E}-04$ \\
673.15 & 100.0000 & 38.464 & $4.9530 \mathrm{E}-04$ \\
673.15 & 150.0000 & 41.701 & $6.0051 \mathrm{E}-04$ \\
673.15 & 200.0000 & 43.992 & $6.6065 \mathrm{E}-04$ \\
\hline
\end{tabular}

Reference: Khitarov, N.I., 1956, The 400 degree isotherm

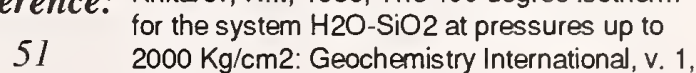
p. $55-61$

Units of original measurements: Temperature in degrees $C$, Pressure in $\mathrm{kg} / \mathrm{cm} 2$, Solubility in $\mathrm{mg} / \mathrm{L}$

Temperature Pressure Density Solubility

\begin{tabular}{crcc}
\multicolumn{1}{c}{$(\mathrm{K})$} & \multicolumn{1}{c}{$(\mathrm{MPa})$} & $(\mathrm{mol} / \mathrm{L})$ & (mole fraction) \\
\hline 623.15 & 294.1995 & 49.344 & $5.4339 \mathrm{E}-04$ \\
673.15 & 58.8399 & 33.802 & $2.9104 \mathrm{E}-04$ \\
673.15 & 98.0665 & 38.305 & $4.2919 \mathrm{E}-04$ \\
673.15 & 147.0998 & 41.547 & $5.7646 \mathrm{E}-04$ \\
673.15 & 196.1330 & 43.835 & $6.5163 \mathrm{E}-04$ \\
673.15 & 235.3596 & 45.307 & $7.3465 \mathrm{E}-04$ \\
673.15 & 274.5862 & 46.574 & $7.7196 \mathrm{E}-04$ \\
673.15 & 294.1995 & 47.148 & $7.8550 \mathrm{E}-04$ \\
673.15 & 343.2328 & 48.453 & $8.4118 \mathrm{E}-04$ \\
673.15 & 348.1361 & 48.574 & $8.5684 \mathrm{E}-04$ \\
673.15 & 392.2660 & 49.609 & $9.0742 \mathrm{E}-04$ \\
723.15 & 294.1995 & 44.903 & $1.2841 \mathrm{E}-03$ \\
\hline
\end{tabular}

Reference: Kitahara, S., 1960, The solubility of quartz in water at high temperatures and pressures: Rev. 52 Phys. Chemistry Japan, v. 30, p. 109-114

Units of original measurements: Temperature in degrees $C$, Pressure in atm, Volume in $\mathrm{mL} / \mathrm{g}$, Solubility in $\mathrm{wt} \%$, "indicates data point is at saturation

Temperature Pressure Density Solubility (K) $\quad(\mathrm{MPa}) \quad(\mathrm{mol} / \mathrm{L}) \quad$ (mole fraction)

$\begin{array}{rrrr}433.15 & 0.6079 & 50.371 & 2.3988 \mathrm{E}-05^{*} \\ 473.15 & 1.5199 & 47.996 & 7.7971 \mathrm{E}-05^{*} \\ 513.15 & 3.3437 & 45.149 & 1.3497 \mathrm{E}-04^{*} \\ 553.15 & 6.3835 & 41.647 & 1.7397 \mathrm{E}-04^{*} \\ 573.15 & 8.8153 & 39.530 & 1.8598 \mathrm{E}-04^{*} \\ 593.15 & 11.4497 & 37.029 & 1.9498 \mathrm{E}-04^{*} \\ 613.15 & 14.6921 & 33.897 & 1.9498 \mathrm{E}-04^{*}\end{array}$


Temperature Pressure Density Solubility (K)

633.15

643.15

653.15

653.15

653.15

653.15

653.15

653.15

653.15

653.15

653.15

653.15

673.15

673.15

673.15

673.15

673.15

673.15

673.15

673.15

673.15

673.15

693.15

693.15

693.15

693.15

693.15

693.15

693.15

693.15

693.15

713.15

713.15

713.15

713.15

713.15

713.15

713.15

713.15

733.15

733.15

733.15

733.15

733.15

733.15

733.15

733.15

753.15

753.15

753.15

753.15
(MPa) (mol/L) (mole fraction)

$\begin{array}{lll}18.6438 & 29.286 & 1.5897 \mathrm{E}-04\end{array}$

$21.075625 .058 \quad 7.4971 \mathrm{E}-05^{*}$

$23.3990 \quad 13.877 \quad 5.3977 \mathrm{E}-05$

$23.5724 \quad 15.860 \quad 6.8973$ E-05

$23.7312 \quad 18.503 \quad 8.9969 E-05$

$23.8961 \quad 20.559 \quad 1.0797 \mathrm{E}-04$

$24.3314 \quad 23.129 \quad 1.3197 \mathrm{E}-04$

$25.1031 \quad 25.231 \quad 1.5897 \mathrm{E}-04$

$27.1334 \quad 27.754 \quad 2.0098 \mathrm{E}-04$

$32.7120 \quad 30.838 \quad 2.6101 \mathrm{E}-04$

$48.4744 \quad 34.693 \quad 3.3608 \mathrm{E}-04$

$65.3194 \quad 37.006 \quad 3.7512 \mathrm{E}-04$

$27.7892 \quad 13.877$

$28.5439 \quad 15.860$

$29.4771 \quad 18.503$

30.3171

31.7689

33.6049

37.2416

45.2020

64.5426

83.8407

32.1272

33.5192

35.3620

36.9723

39.5247

42.4728

47.6931

57.9625

80.7927

36.4349

38.5050

41.3027

43.7353

47.4781

51.5615

58.3532

70.9060

40.7098

43.4862

47.2835

50.5772

55.5495

60.7813

69.1498

83.9788

44.9514

48.4549

53.2860

57.4668
20.559

23.129

25.231

27.754

30.838

34.693

37.006

13.877

15.860

18.503

20.559

23.129

25.231

27.754

30.838

34.693

13.877

15.860

18.503

20.559

23.129

25.231

27.754

30.838

13.877

15.860

18.503

20.559

23.129

25.231

27.754

30.838

13.877

15.860

18.503

20.559
6.5974E-05

8.3970E-05

1.0797E-04

1.2897E-04

1.5597E-04

1.9198E-04

2.4000E-04

3.0905E-04

3.9314E-04

4.4121E-04

8.0970E-05

1.0197E-04

1.3197E-04

1.5597E-0 4

1.8898E-04

2.2799E-04

2.8203E-04

3.6010E-04

4.7126E-04

9.8968E-05

1.2297E-04

1.5897E-04

1.8598E-04

2.2499E-04

2.7002E-04

3.3007E-04

4.2018E-04

1.1997E-04

1.4697E-04

1.8898E-0 4

2.2499E-0 4

2.7602E-0 4

3.2407E-0 4

3.9615E-04

4.9530E-04

1.4097E-04

1.7697E-04

2.2499E-04

2.7002E-0 4

Temperature Pressure Density Solubility

\begin{tabular}{llcc}
$(\mathrm{K})$ & $(\mathrm{MPa})$ & $(\mathrm{mol} / \mathrm{L})$ & $($ mole fraction $)$ \\
\hline 753.15 & 63.6913 & 23.129 & $3.3307 \mathrm{E}-04$ \\
753.15 & 70.0847 & 25.231 & $3.9014 \mathrm{E}-04$ \\
753.15 & 80.0410 & 27.754 & $4.7426 \mathrm{E}-04$ \\
773.15 & 49.1580 & 13.877 & $1.6497 \mathrm{E}-04$ \\
773.15 & 53.4043 & 15.860 & $2.0999 \mathrm{E}-04$ \\
773.15 & 59.2948 & 18.503 & $2.7002 \mathrm{E}-04$ \\
773.15 & 64.3815 & 20.559 & $3.1806 \mathrm{E}-04$ \\
773.15 & 71.8765 & 23.129 & $3.9014 \mathrm{E}-04$ \\
773.15 & 79.4439 & 25.231 & $4.6525 \mathrm{E}-04$ \\
773.15 & 90.9985 & 27.754 & $5.5541 \mathrm{E}-04$ \\
\hline
\end{tabular}

Reference: Kennedy, G.C., Wasserburg, G.J., Heard, H.C.

and Newton, R.C., 1962, The upper three phase

53 region in the system $\mathrm{SiO} 2-\mathrm{H} 2 \mathrm{O}:$ American Journal of Science, v. 260, p. 501-521

Units of original measurements: Temperature in degrees $C$,

Pressure in kbar, Solubility in wt\%

Temperature Pressure Density Solubility

(K)

1321.15

1323.15

1326.15

1326.15

1326.15

1326.15

1326.15

1327.15

1327.15

1328.15

1329.15

1331.15

1333.15

1334.15

1334.15

1334.15

1334.15

1334.15

1339.15

1341.15

1342.15

1353.15

1353.15

1353.15

1353.15

1353.15

1354.15

1359.15

1382.15
(MPa) (mol/L) (mole fraction) $800.0000 \quad 40.261 \quad 1.1100 \mathrm{E}-01$ $800.0000 \quad 40.221 \quad 1.0028 \mathrm{E}-01$ $800.0000 \quad 40.161 \quad 1.0629 \mathrm{E}-01$ $900.0000 \quad 42.154 \quad 2.0938 \mathrm{E}-01$ $950.0000 \quad 43.064 \quad 2.8441 \mathrm{E}-01$ $950.0000 \quad 43.064 \quad 3.2108 \mathrm{E}-01$ $950.0000 \quad 43.064 \quad 3.2663 \mathrm{E}-01$ $600.0000 \quad 35.225 \quad 6.2146 \mathrm{E}-02$ $900.0000 \quad 42.135 \quad 2.1406 \mathrm{E}-01$ $950.0000 \quad 43.026 \quad 2.3712 \mathrm{E}-01$ $600.0000 \quad 35.182 \quad 4.9132 \mathrm{E}-02$ $600.0000 \quad 35.140 \quad 6.5311 \mathrm{E}-02$ $900.0000 \quad 42.020 \quad 1.4165 \mathrm{E}-01$ $800.0000 \quad 40.002 \quad 1.0769 E-01$ $800.0000 \quad 40.002 \quad 8.0924 \mathrm{E}-02$ $800.0000 \quad 40.002 \quad 8.7797 \mathrm{E}-02$ $800.0000 \quad 40.002 \quad 1.0489 E-01$ $800.0000 \quad 40.002 \quad 1.0028 \mathrm{E}-01$ $700.0000 \quad 37.617 \quad 8.3052 \mathrm{E}-02$ $600.0000 \quad 34.928 \quad 7.2176 \mathrm{E}-02$ $500.0000 \quad 31.789 \quad 4.3973 \mathrm{E}-02$ $400.0000 \quad 27.802 \quad 3.2588 \mathrm{E}-02$ $400.0000 \quad 27.802 \quad 3.1203 \mathrm{E}-02$ $400.0000 \quad 27.802 \quad 3.0858 \mathrm{E}-02$ $400.0000 \quad 27.802 \quad 3.1548 \mathrm{E}-02$ $400.0000 \quad 27.802 \quad 3.5734 \mathrm{E}-02$ $400.0000 \quad 27.781 \quad 3.3981 \mathrm{E}-02$ $300.0000 \quad 22.991 \quad 2.4068 \mathrm{E}-02$ $200.0000 \quad 16.500 \quad 1.7801 \mathrm{E}-02$ 
Reference: Morey, G.W., Fournier, R.O., and Rowe, J.J., temperature interval from 25 to $300 \mathrm{C}$ : Geochimica et Cosmochimica Acta, v. 26, p. 1029-1043

Units of original measurements: Temperature in degrees $C$, Pressure in atm, Solubility in ppm

\begin{tabular}{cccc}
$\begin{array}{c}\text { Temperature } \\
(\mathrm{K})\end{array}$ & $\begin{array}{c}\text { Pressure } \\
\text { (MPa) }\end{array}$ & $\begin{array}{c}\text { Density } \\
\text { (mol/L) }\end{array}$ & $\begin{array}{c}\text { Solubility } \\
\text { (mole fraction) }\end{array}$ \\
\hline 318.15 & 101.3250 & 57.185 & $6.2966 \mathrm{E}-06$ \\
318.15 & 101.3250 & 57.185 & $5.6969 \mathrm{E}-06$ \\
318.15 & 101.3250 & 57.185 & $5.6969 \mathrm{E}-06$ \\
348.15 & 101.3250 & 56.346 & $1.0794 \mathrm{E}-05$ \\
348.15 & 101.3250 & 56.346 & $1.1394 \mathrm{E}-05$ \\
363.15 & 101.3250 & 55.864 & $1.7091 \mathrm{E}-05$ \\
363.15 & 101.3250 & 55.864 & $1.5892 \mathrm{E}-05$ \\
373.15 & 101.3250 & 55.522 & $2.0689 \mathrm{E}-05$ \\
373.15 & 101.3250 & 55.522 & $1.9790 \mathrm{E}-05$ \\
373.15 & 101.3250 & 55.522 & $2.0390 \mathrm{E}-05$ \\
378.15 & 101.3250 & 55.346 & $2.4288 \mathrm{E}-05$ \\
383.15 & 101.3250 & 55.165 & $2.6987 \mathrm{E}-05$ \\
389.15 & 101.3250 & 54.943 & $3.1785 \mathrm{E}-05$ \\
393.15 & 101.3250 & 54.792 & $3.4184 \mathrm{E}-05$ \\
393.15 & 101.3250 & 54.792 & $3.3584 \mathrm{E}-05$ \\
393.15 & 101.3250 & 54.792 & $3.3284 \mathrm{E}-05$ \\
398.15 & 101.3250 & 54.600 & $3.5983 \mathrm{E}-05$ \\
398.15 & 101.3250 & 54.600 & $3.5983 \mathrm{E}-05$ \\
410.15 & 101.3250 & 54.125 & $4.4080 \mathrm{E}-05$ \\
410.15 & 101.3250 & 54.125 & $4.3180 \mathrm{E}-05$ \\
410.15 & 101.3250 & 54.125 & $4.2880 \mathrm{E}-05$ \\
418.15 & 101.3250 & 53.797 & $5.1877 \mathrm{E}-05$ \\
423.15 & 101.3250 & 53.588 & $5.3077 \mathrm{E}-05$ \\
433.15 & 101.3250 & 53.159 & $6.2374 \mathrm{E}-05$ \\
433.15 & 101.3250 & 53.159 & $6.2974 \mathrm{E}-05$ \\
433.15 & 101.3250 & 53.159 & $6.1475 \mathrm{E}-05$ \\
433.15 & 101.3250 & 53.159 & $6.1175 \mathrm{E}-05$ \\
443.15 & 101.3250 & 52.716 & $6.7773 \mathrm{E}-05$ \\
458.15 & 101.3250 & 52.028 & $7.8871 \mathrm{E}-05$ \\
463.15 & 101.3250 & 51.793 & $8.6369 \mathrm{E}-05$ \\
473.15 & 101.3250 & 51.311 & $9.8968 \mathrm{E}-05$ \\
473.15 & 101.3250 & 51.311 & $9.8368 \mathrm{E}-05$ \\
498.15 & 101.3250 & 50.051 & $1.3317 \mathrm{E}-04$ \\
519.15 & 101.3250 & 48.927 & $1.6737 \mathrm{E}-04$ \\
538.15 & 101.3250 & 47.858 & $2.0098 \mathrm{E}-04$ \\
543.15 & 101.3250 & 47.568 & $2.1899 \mathrm{E}-04$ \\
546.15 & 101.3250 & 47.393 & $2.3250 \mathrm{E}-04$ \\
570.15 & 101.3250 & 45.938 & $2.6702 \mathrm{E}-04$ \\
570.15 & 101.3250 & 45.938 & $2.6702 \mathrm{E}-04$ \\
573.15 & 101.3250 & 45.750 & $2.7602 \mathrm{E}-04$ \\
573.15 & 101.3250 & 45.750 & $2.7723 \mathrm{E}-04$ \\
\hline & & & \\
\hline
\end{tabular}

Reference: Weill, D.F. and Fyfe, W.S., 1964, The solubility 55 of quartz in $\mathrm{H} 2 \mathrm{O}$ in the range $1000-4000$ bars and 400-550 C: Geochimica et Cosmochimica Acta, v. 28 , p. $1243-1255$

Units of original measurements: Temperature in degrees $C$, Pressure in bar, Solubility in wt\%

Temperature Pressure Density Solubility (K)

$\begin{array}{llll}673.15 & 100.0000 & 38.464 & 5.1032 \mathrm{E}-04 \\ 673.15 & 125.0000 & 40.255 & 5.2235 \mathrm{E}-04 \\ 673.15 & 125.0000 & 40.255 & 5.4038 \mathrm{E}-04 \\ 673.15 & 125.0000 & 40.255 & 5.5541 \mathrm{E}-04 \\ 673.15 & 125.0000 & 40.255 & 5.5241 \mathrm{E}-04 \\ 673.15 & 125.0000 & 40.255 & 5.6142 \mathrm{E}-04 \\ 673.15 & 125.0000 & 40.255 & 4.2018 \mathrm{E}-04 \\ 673.15 & 150.0000 & 41.701 & 6.2456 \mathrm{E}-04 \\ 673.15 & 175.0000 & 42.925 & 6.9675 \mathrm{E}-04 \\ 673.15 & 200.0000 & 43.992 & 7.4488 \mathrm{E}-04 \\ 673.15 & 225.0000 & 44.942 & 7.7497 \mathrm{E}-04 \\ 673.15 & 250.0000 & 45.800 & 8.0506 \mathrm{E}-04 \\ 673.15 & 275.0000 & 46.586 & 8.2312 \mathrm{E}-04 \\ 673.15 & 300.0000 & 47.312 & 8.2914 \mathrm{E}-04 \\ 673.15 & 325.0000 & 47.987 & 8.4419 \mathrm{E}-04 \\ 673.15 & 400.0000 & 49.780 & 8.7129 \mathrm{E}-04 \\ 723.15 & 100.0000 & 34.091 & 6.4862 \mathrm{E}-04 \\ 723.15 & 125.0000 & 36.548 & 7.8099 \mathrm{E}-04 \\ 723.15 & 150.0000 & 38.416 & 8.4419 \mathrm{E}-04 \\ 723.15 & 175.0000 & 39.936 & 9.4656 \mathrm{E}-04 \\ 723.15 & 200.0000 & 41.227 & 9.9475 \mathrm{E}-04 \\ 723.15 & 225.0000 & 42.353 & 1.0701 \mathrm{E}-03 \\ 723.15 & 250.0000 & 43.355 & 1.1303 \mathrm{E}-03 \\ 723.15 & 275.0000 & 44.261 & 1.1906 \mathrm{E}-03 \\ 723.15 & 300.0000 & 45.089 & 1.2660 \mathrm{E}-03 \\ 723.15 & 350.0000 & 46.562 & 1.3626 \mathrm{E}-03 \\ 723.15 & 400.0000 & 47.850 & 1.4893 \mathrm{E}-03 \\ 773.15 & 100.0000 & 29.324 & 7.2081 \mathrm{E}-04 \\ 773.15 & 150.0000 & 34.971 & 1.1183 \mathrm{E}-03 \\ 773.15 & 200.0000 & 38.380 & 1.3716 \mathrm{E}-03 \\ 773.15 & 250.0000 & 40.865 & 1.5195 \mathrm{E}-03 \\ 773.15 & 300.0000 & 42.841 & 1.7099 \mathrm{E}-03 \\ 773.15 & 350.0000 & 44.492 & 1.8429 \mathrm{E}-03 \\ 773.15 & 400.0000 & 45.917 & 2.0123 \mathrm{E}-03 \\ 823.15 & 100.0000 & 24.676 & 7.8099 \mathrm{E}-04 \\ 823.15 & 150.0000 & 31.497 & 1.3475 \mathrm{E}-03 \\ 823.15 & 200.0000 & 35.514 & 1.7129 \mathrm{E}-03 \\ 823.15 & 250.0000 & 38.368 & 1.9760 \mathrm{E}-03 \\ 823.15 & 300.0000 & 40.595 & 2.2303 \mathrm{E}-03 \\ 823.15 & 400.0000 & 43.997 & 2.6519 \mathrm{E}-03 \\ 898.15 & 100.0000 & 19.182 & 8.2914 \mathrm{E}-04 \\ 898.15 & 100.0000 & 19.182 & 8.2312 \mathrm{E}-04 \\ 898.15 & 100.0000 & 19.182 & 7.9904 \mathrm{E}-04 \\ 898.15 & 100.0000 & 19.182 & 7.7497 \mathrm{E}-04 \\ 898.15 & 100.0000 & 19.182 & 7.0577 \mathrm{E}-04 \\ 898.15 & 100.0000 & 19.182 & 5.7044 \mathrm{E}-04\end{array}$


Temperature Pressure Density Solubility

\begin{tabular}{cccc}
$(\mathrm{K})$ & $(\mathrm{MPa})$ & $(\mathrm{mol} / \mathrm{L})$ & $($ mole fraction $)$ \\
\hline 898.15 & 100.0000 & 19.182 & $8.0506 \mathrm{E}-04$ \\
898.15 & 100.0000 & 19.182 & $8.0807 \mathrm{E}-04$ \\
\hline
\end{tabular}

Reference: Anderson, G.M. and Bumham, C.W., 1965, The solubility of quartz in supercritical water:

56 American Journal of Science, v. 263, p. 494-511

Units of original measurements: Temperature in degrees $C$,

Pressure in kbar, Solubility in wt\%

Temperature Pressure Density Solubility

\begin{tabular}{cccc}
$(\mathrm{K})$ & $(\mathrm{MPa})$ & $(\mathrm{mol} / \mathrm{L})$ & (mole fraction) \\
\hline 773.15 & 100.0000 & 29.324 & $7.2081 \mathrm{E}-04$ \\
773.15 & 150.0000 & 34.971 & $1.0821 \mathrm{E}-03$ \\
773.15 & 150.0000 & 34.971 & $1.0821 \mathrm{E}-03$ \\
773.15 & 200.0000 & 38.380 & $1.2329 \mathrm{E}-03$ \\
773.15 & 300.0000 & 42.841 & $1.6857 \mathrm{E}-03$ \\
773.15 & 400.0000 & 45.917 & $1.9881 \mathrm{E}-03$ \\
773.15 & 500.0000 & 48.309 & $2.2606 \mathrm{E}-03$ \\
773.15 & 600.0000 & 50.287 & $2.4425 \mathrm{E}-03$ \\
773.15 & 600.0000 & 50.287 & $2.4728 \mathrm{E}-03$ \\
773.15 & 750.0000 & 52.759 & $2.7764 \mathrm{E}-03$ \\
773.15 & 830.0000 & 53.906 & $2.7460 \mathrm{E}-03$ \\
773.15 & 860.0000 & 54.312 & $2.8979 \mathrm{E}-03$ \\
873.15 & 100.0000 & 20.772 & $7.8099 \mathrm{E}-04$ \\
873.15 & 150.0000 & 28.192 & $1.5648 \mathrm{E}-03$ \\
873.15 & 150.0000 & 28.192 & $1.5346 \mathrm{E}-03$ \\
873.15 & 150.0000 & 28.192 & $1.4139 \mathrm{E}-03$ \\
873.15 & 200.0000 & 32.714 & $2.1697 \mathrm{E}-03$ \\
873.15 & 300.0000 & 38.379 & $2.8979 \mathrm{E}-03$ \\
873.15 & 300.0000 & 38.379 & $3.0499 \mathrm{E}-03$ \\
873.15 & 400.0000 & 42.105 & $3.5065 \mathrm{E}-03$ \\
873.15 & 400.0000 & 42.105 & $3.5675 \mathrm{E}-03$ \\
873.15 & 400.0000 & 42.105 & $3.4761 \mathrm{E}-03$ \\
873.15 & 500.0000 & 44.909 & $4.1169 \mathrm{E}-03$ \\
873.15 & 500.0000 & 44.909 & $3.8115 \mathrm{E}-03$ \\
873.15 & 600.0000 & 47.176 & $4.4228 \mathrm{E}-03$ \\
873.15 & 600.0000 & 47.176 & $4.6065 \mathrm{E}-03$ \\
873.15 & 620.0000 & 47.583 & $4.6678 \mathrm{E}-03$ \\
873.15 & 740.0000 & 49.785 & $5.2814 \mathrm{E}-03$ \\
873.15 & 745.0000 & 49.869 & $4.9130 \mathrm{E}-03$ \\
973.15 & 150.0000 & 22.706 & $1.9276 \mathrm{E}-03$ \\
973.15 & 150.0000 & 22.706 & $1.9276 \mathrm{E}-03$ \\
973.15 & 200.0000 & 27.676 & $2.6853 \mathrm{E}-03$ \\
973.15 & 200.0000 & 27.676 & $3.0195 \mathrm{E}-03$ \\
973.15 & 290.0000 & 33.650 & $4.4840 \mathrm{E}-03$ \\
973.15 & 300.0000 & 34.172 & $4.7904 \mathrm{E}-03$ \\
973.15 & 400.0000 & 38.463 & $5.0051 \mathrm{E}-03$ \\
973.15 & 485.0000 & 41.225 & $6.8233 \mathrm{E}-03$ \\
973.15 & 500.0000 & 41.655 & $6.6686 \mathrm{E}-03$ \\
973.15 & 605.0000 & 44.316 & $8.0647 \mathrm{E}-03$ \\
973.15 & 750.0000 & 47.273 & $9.7518 \mathrm{E}-03$ \\
973.15 & 800.0000 & 48.158 & $9.9401 \mathrm{E}-03$ \\
973.15 & 880.0000 & 49.465 & $1.0947 \mathrm{E}-02$
\end{tabular}

Temperature Pressure Density Solubility

\begin{tabular}{|c|c|c|c|}
\hline$(\mathrm{K})$ & (MPa) & $(\mathrm{mol} / \mathrm{L})$ & (mole fraction) \\
\hline 973.15 & 985.0000 & 51.014 & 1.2212E-02 \\
\hline 1073.15 & 200.0000 & 23.687 & 4.0864E-03 \\
\hline 1073.15 & 200.0000 & 23.687 & 4.1169E-03 \\
\hline 1073.15 & 300.0000 & 30.461 & 7.0400E-03 \\
\hline 1073.15 & 400.0000 & 35.110 & $9.0629 \mathrm{E}-03$ \\
\hline 1073.15 & 400.0000 & 35.110 & $9.3758 \mathrm{E}-03$ \\
\hline 1073.15 & 500.0000 & 38.609 & 1.1294E-02 \\
\hline 1073.15 & 600.0000 & 41.399 & 1.3836E-02 \\
\hline 1073.15 & 600.0000 & 41.399 & 1.3229E-02 \\
\hline 1073.15 & 770.0000 & 45.134 & 1.6407E-02 \\
\hline 1173.15 & 200.0000 & 20.676 & 5.2814E-03 \\
\hline 1173.15 & 200.0000 & 20.676 & 4.9744E-03 \\
\hline 1173.15 & 200.0000 & 20.676 & 5.1893E-03 \\
\hline 1173.15 & 210.0000 & 21.465 & 5.5582E-03 \\
\hline 1173.15 & 300.0000 & 27.354 & $9.8459 E-03$ \\
\hline 1173.15 & 305.0000 & 27.629 & $1.0254 \mathrm{E}-02$ \\
\hline 1173.15 & 400.0000 & 32.137 & 1.3644E-02 \\
\hline 1173.15 & 510.0000 & 36.148 & 1.9827E-02 \\
\hline 1173.15 & 520.0000 & 36.466 & 1.7443E-02 \\
\hline 1173.15 & 550.0000 & 37.383 & 1.8746E-02 \\
\hline 1173.15 & 560.0000 & 37.676 & 1.5922E-02 \\
\hline 1173.15 & 590.0000 & 38.524 & 2.0814E-02 \\
\hline 1173.15 & 730.0000 & 41.943 & $2.9142 E-02$ \\
\hline 1173.15 & 740.0000 & 42.159 & 2.5915E-02 \\
\hline 1173.15 & 740.0000 & 42.159 & 2.3801E-02 \\
\hline 1173.15 & 750.0000 & 42.372 & 2.7404E-02 \\
\hline 1173.15 & 830.0000 & 43.974 & 2.6692E-02 \\
\hline
\end{tabular}

Reference: Semenova, A.I. and Tsiklis, D.S., 1970,

Solubility of silicon dioxide in steam at high pressures and temperatures: Russian Journal of Physical Chemistry, v. 44, n. 10, p. 1420-1422

Units of original measurements: Temperature in degrees $C$, Pressure in bar, Solubility in wt\%, Original data in graphical format

Temperature Pressure Density Solubility

\begin{tabular}{rrrr}
\multicolumn{1}{c}{$(\mathrm{K})$} & \multicolumn{1}{c}{$(\mathrm{MPa})$} & $(\mathrm{mol} / \mathrm{L})$ & $($ mole fraction $)$ \\
\hline 873.15 & 96.9767 & 20.168 & $8.8902 \mathrm{E}-05$ \\
873.15 & 145.5385 & 27.683 & $3.4792 \mathrm{E}-04$ \\
873.15 & 165.3488 & 29.785 & $7.8361 \mathrm{E}-04$ \\
873.15 & 170.7692 & 30.297 & $7.6180 \mathrm{E}-04$ \\
873.15 & 173.2308 & 30.523 & $1.1328 \mathrm{E}-03$ \\
873.15 & 192.9231 & 32.176 & $8.9267 \mathrm{E}-04$ \\
873.15 & 196.0000 & 32.413 & $1.2857 \mathrm{E}-03$ \\
873.15 & 209.3023 & 33.384 & $1.2480 \mathrm{E}-03$ \\
873.15 & 209.5385 & 33.400 & $1.6686 \mathrm{E}-03$ \\
873.15 & 229.8461 & 34.731 & $1.5044 \mathrm{E}-03$ \\
873.15 & 232.9231 & 34.919 & $2.0960 \mathrm{E}-03$ \\
873.15 & 251.8605 & 36.012 & $1.7911 \mathrm{E}-03$ \\
873.15 & 259.5349 & 36.425 & $1.7552 \mathrm{E}-03$ \\
873.15 & 260.2326 & 36.462 & $2.1319 \mathrm{E}-03$ \\
873.15 & 260.6154 & 36.482 & $2.2496 \mathrm{E}-03$ \\
873.15 & 268.0000 & 36.863 & $2.1618 \mathrm{E}-03$
\end{tabular}




\begin{tabular}{cccc}
$\begin{array}{c}\text { Temperature } \\
(\mathrm{K})\end{array}$ & $\begin{array}{c}\text { Pressure } \\
(\mathrm{MPa})\end{array}$ & $\begin{array}{c}\text { Density } \\
(\mathrm{mol} / \mathrm{L})\end{array}$ & $\begin{array}{c}\text { Solubility } \\
\text { (mole fraction) }\end{array}$ \\
\hline 873.15 & 272.9231 & 37.111 & $2.0412 \mathrm{E}-03$ \\
873.15 & 300.0000 & 38.379 & $2.6412 \mathrm{E}-03$ \\
973.15 & 102.5581 & 16.070 & $5.9256 \mathrm{E}-06$ \\
973.15 & 124.6154 & 19.416 & $1.0864 \mathrm{E}-05$ \\
973.15 & 160.3077 & 23.877 & $6.6370 \mathrm{E}-04$ \\
973.15 & 177.9070 & 25.687 & $1.7791 \mathrm{E}-03$ \\
973.15 & 195.3846 & 27.284 & $1.9425 \mathrm{E}-03$ \\
973.15 & 196.7442 & 27.400 & $2.1319 \mathrm{E}-03$ \\
973.15 & 223.0769 & 29.484 & $2.4473 \mathrm{E}-03$ \\
973.15 & 238.6046 & 30.576 & $3.0014 \mathrm{E}-03$ \\
973.15 & 250.7692 & 31.372 & $3.3502 \mathrm{E}-03$ \\
973.15 & 272.0930 & 32.660 & $3.4767 \mathrm{E}-03$ \\
973.15 & 301.3954 & 34.243 & $3.7720 \mathrm{E}-03$ \\
1073.15 & 115.1163 & 14.750 & $5.9256 \mathrm{E}-06$ \\
1073.15 & 141.8461 & 17.958 & $5.2209 \mathrm{E}-04$ \\
1073.15 & 144.3077 & 18.236 & $7.0730 \mathrm{E}-04$ \\
1073.15 & 145.8140 & 18.405 & $1.0574 \mathrm{E}-03$ \\
1073.15 & 166.0465 & 20.550 & $1.0871 \mathrm{E}-03$ \\
1073.15 & 214.8837 & 24.900 & $2.3595 \mathrm{E}-03$ \\
1073.15 & 230.4615 & 26.079 & $3.6815 \mathrm{E}-03$ \\
1073.15 & 252.0000 & 27.575 & $4.1129 \mathrm{E}-03$ \\
1073.15 & 253.2308 & 27.657 & $4.2569 \mathrm{E}-03$ \\
1073.15 & 258.8372 & 28.021 & $3.7117 \mathrm{E}-03$ \\
1073.15 & 305.5814 & 30.763 & $4.9450 \mathrm{E}-03$ \\
1073.15 & 327.2093 & 31.879 & $5.6556 \mathrm{E}-03$ \\
1173.15 & 172.3256 & 18.320 & $1.7970 \mathrm{E}-03$ \\
1173.15 & 186.2791 & 19.540 & $2.2636 \mathrm{E}-03$ \\
1173.15 & 216.2791 & 21.945 & $3.5008 \mathrm{E}-03$ \\
1173.15 & 245.5814 & 24.034 & $4.8420 \mathrm{E}-03$ \\
\hline & & & \\
\hline
\end{tabular}

Reference: Crerar, D.A. and Anderson, G.M., 1971,

Solubility and solvation reactions of quartz in 58 dilute hydrothermal solutions: Chemical Geology, v. 8, p. $107-122$

Units of original measurements: Temperature in degrees $C$ Solubility in ppm of SiO2, all data points are at saturation

Temperature Pressure Density Solubility

\begin{tabular}{cccc}
$(\mathrm{K})$ & $(\mathrm{MPa})$ & $(\mathrm{mol} / \mathrm{L})$ & (mole fraction) \\
\hline 452.15 & 0.9799 & 49.295 & $5.1278 \mathrm{E}-05$ \\
452.15 & 0.9799 & 49.295 & $5.1877 \mathrm{E}-05$ \\
453.15 & 1.0028 & 49.236 & $4.8878 \mathrm{E}-05$ \\
454.15 & 1.0261 & 49.177 & $5.0318 \mathrm{E}-05$ \\
502.15 & 2.7462 & 45.986 & $1.2180 \mathrm{E}-04$ \\
543.15 & 5.5030 & 42.601 & $1.5537 \mathrm{E}-04$ \\
546.15 & 5.7659 & 42.321 & $1.6089 \mathrm{E}-04$ \\
546.15 & 5.7659 & 42.321 & $1.5483 \mathrm{E}-04$ \\
546.15 & 5.7659 & 42.321 & $1.5738 \mathrm{E}-04$ \\
546.15 & 5.7659 & 42.321 & $1.5099 \mathrm{E}-04$ \\
547.15 & 5.8556 & 42.226 & $1.5438 \mathrm{E}-04$ \\
548.15 & 5.9464 & 42.131 & $1.5039 \mathrm{E}-04$ \\
549.15 & 6.0382 & 42.036 & $1.5546 \mathrm{E}-04$ \\
549.15 & 6.0382 & 42.036 & $1.5372 \mathrm{E}-04$
\end{tabular}

\begin{tabular}{crrr}
$\begin{array}{c}\text { Temperature } \\
(\mathrm{K})\end{array}$ & $\begin{array}{c}\text { Pressure } \\
(\mathrm{MPa})\end{array}$ & $\begin{array}{c}\text { Density } \\
(\mathrm{mol} / \mathrm{L})\end{array}$ & $\begin{array}{c}\text { Solubility } \\
\text { (mole fraction })\end{array}$ \\
\hline 549.15 & 6.0382 & 42.036 & $1.5606 \mathrm{E}-04$ \\
549.15 & 6.0382 & 42.036 & $1.5861 \mathrm{E}-04$ \\
550.15 & 6.1312 & 41.939 & $1.5597 \mathrm{E}-04$ \\
550.15 & 6.1312 & 41.939 & $1.5588 \mathrm{E}-04$ \\
575.15 & 8.8325 & 39.299 & $1.8205 \mathrm{E}-04$ \\
578.15 & 92094 & 38.947 & $1.8217 \mathrm{E}-04$ \\
578.15 & 9.2094 & 38.947 & $1.8157 \mathrm{E}-04$ \\
594.15 & 11.4345 & 36.891 & $1.9225 \mathrm{E}-04$ \\
594.15 & 11.4345 & 36.891 & $1.8574 \mathrm{E}-04$ \\
596.15 & 11.7396 & 36.609 & $2.0287 \mathrm{E}-04$ \\
596.15 & 11.7396 & 36.609 & $1.8721 \mathrm{E}-04$ \\
596.15 & 11.7396 & 36.609 & $1.9123 \mathrm{E}-04$ \\
597.15 & 11.8945 & 36.466 & $1.9951 \mathrm{E}-04$ \\
599.15 & 12.2091 & 36.174 & $1.8931 \mathrm{E}-04$ \\
599.15 & 12.2091 & 36.174 & $1.8940 \mathrm{E}-04$ \\
602.15 & 12.6933 & 35.723 & $1.9222 \mathrm{E}-04$ \\
602.15 & 12.6933 & 35.723 & $1.8661 \mathrm{E}-04$ \\
\hline
\end{tabular}

Reference: Hemley, J.J., Montoya, J.W., Marinenko, J.W and Luce, R.W., 1980, Equilibria in the system

59 Al2O3-SiO2- $-\mathrm{H}_{2} \mathrm{O}$ and some general implications for alteration/mineralization processes: Economic Geology, v. 75 , p. 210-228

Units of original measurements: Temperature in degrees $C$, Pressure in kbar, Solubility in molality

Temperature Pressure Density Solubility (K) 473.15 (MPa) (mol/L) (mole fraction) 473.15 1.5550 47.996 8.4510E-05 473.15 523.15 523.15 523.15 573.15 573.15 573.15 623.15 623.15 623.15 673.15 673.15 723.15 723.15 773.15 100.0000 51.275 9.7031E-05 200.0000 53.643 1.0639E-04 $3.9760 \quad 44.345 \quad 1.3706 \mathrm{E}-04$ $100.0000 \quad 48.662 \quad 1.6862 \mathrm{E}-04$ $200.0000 \quad 51.476 \quad 1.9811 \mathrm{E}-04$ $8.5880 \quad 39.530 \quad 2.0273 E-04$ $100.0000 \quad 45.693 \quad 2.6724 \mathrm{E}-04$ $200.0000 \quad 49.136 \quad 3.2878 E-04$ $16.5300 \quad 31.901 \quad 2.2746 \mathrm{E}-04$ $100.0000 \quad 42.316 \quad 3.9528 \mathrm{E}-04$ $200.0000 \quad 46.636 \quad 5.2108 \mathrm{E}-04$ $100.0000 \quad 38.464 \quad 5.4564 \mathrm{E}-04$ $200.0000 \quad 43.992 \quad 7.3605 E-04$ $100.0000 \quad 34.091 \quad 6.8692 \mathrm{E}-04$ $200.0000 \quad 41.227 \quad 9.9291 \mathrm{E}-04$ $100.0000 \quad 29.324 \quad 8.4510 \mathrm{E}-04$ $\begin{array}{llll}773.15 & 200.0000 & 38.380 & 1.3706 \mathrm{E}-03\end{array}$ 
Reference: Ragnarsdottir, K. V. and Walther, J.V., 1983,

Pressure sensitive "silica geothermometer" determined from quartz solubility experiments at 250 C: Geochimica et Cosmochimica Acta, v. 47 , p. $941-946$

Units of original measurements: Temperature in degrees $C$, Pressure in bar, Solubility in molality

Temperature Pressure Density Solubility

\begin{tabular}{crrr}
$(\mathrm{K})$ & \multicolumn{1}{c}{$(\mathrm{MPa})$} & $(\mathrm{mol} / \mathrm{L})$ & (mole fraction) \\
\hline 523.15 & 25.0000 & 45.568 & $1.3539 \mathrm{E}-04$ \\
523.15 & 25.0000 & 45.568 & $1.3415 \mathrm{E}-04$ \\
523.15 & 25.0000 & 45.568 & $1.3477 \mathrm{E}-04$ \\
523.15 & 50.0000 & 46.761 & $1.4845 \mathrm{E}-04$ \\
523.15 & 50.0000 & 46.761 & $1.4641 \mathrm{E}-04$ \\
523.15 & 100.0000 & 48.662 & $1.6352 \mathrm{E}-04$ \\
523.15 & 100.0000 & 48.662 & $1.6694 \mathrm{E}-04$ \\
\hline
\end{tabular}

Reference: Walther, J.V. and Orville, P.M., 1983, The extraction-quench technique for determination of the thermodynamic properties of solute complexes: application to quartz solubility in fluid mixtures: American Mineralogist, v. 68, p. 731741

Units of original measurements: Temperature in degrees $C$, Pressure in bar, Solubility in molality

Temperature Pressure Density Solubility

\begin{tabular}{cccc}
$(\mathrm{K})$ & $(\mathrm{MPa})$ & $(\mathrm{mol} / \mathrm{L})$ & (mole fraction) \\
\hline 623.15 & 100.0000 & 24.676 & $3.9762 \mathrm{E}-04$ \\
623.15 & 200.0000 & 46.636 & $5.5005 \mathrm{E}-04$ \\
673.15 & 100.0000 & 29.324 & $5.1572 \mathrm{E}-04$ \\
673.15 & 200.0000 & 43.992 & $7.3000 \mathrm{E}-04$ \\
673.15 & 200.0000 & 43.992 & $7.2665 \mathrm{E}-04$ \\
673.15 & 200.0000 & 43.992 & $7.3506 \mathrm{E}-04$ \\
698.15 & 200.0000 & 42.623 & $8.6152 \mathrm{E}-04$ \\
723.15 & 200.0000 & 41.227 & $1.0476 \mathrm{E}-03$ \\
723.15 & 200.0000 & 41.227 & $1.0097 \mathrm{E}-03$ \\
733.15 & 100.0000 & 33.159 & $7.1012 \mathrm{E}-04$ \\
748.15 & 200.0000 & 39.811 & $1.1302 \mathrm{E}-03$ \\
773.15 & 200.0000 & 38.380 & $1.2885 \mathrm{E}-03$ \\
773.15 & 200.0000 & 38.380 & $1.2505 \mathrm{E}-03$ \\
773.15 & 100.0000 & 38.464 & $7.3845 \mathrm{E}-04$ \\
798.15 & 200.0000 & 36.945 & $1.5066 \mathrm{E}-03$ \\
798.15 & 200.0000 & 36.945 & $1.4029 \mathrm{E}-03$ \\
798.15 & 200.0000 & 36.945 & $1.4825 \mathrm{E}-03$ \\
798.15 & 200.0000 & 36.945 & $1.4355 \mathrm{E}-03$ \\
823.15 & 200.0000 & 35.514 & $1.5847 \mathrm{E}-03$ \\
823.15 & 100.0000 & 42.316 & $7.9304 \mathrm{E}-04$ \\
\hline
\end{tabular}

Reference: Manning, C.E., 1994, The solubility of quartz in $\mathrm{H} 2 \mathrm{O}$ in the lower crust and upper mantle:

62 Geochimica et Cosmochimica Acta, v. 58, n. 22 , p. $4831-4839$

Units of original measurements: Temperature in degrees $C$, Pressure in kbar, Solubility in wt\%

Temperature Pressure Density Solubility $(\mathrm{K}) \quad(\mathrm{MPa}) \quad(\mathrm{mol} / \mathrm{L}) \quad$ (mole fraction) $\begin{array}{llll}773.15 & 500.0000 & 48.309 & 2.3546 \mathrm{E}-03\end{array}$
Temperature Pressure Density Solubility

\begin{tabular}{cccc}
$(\mathrm{K})$ & $(\mathrm{MPa})$ & $(\mathrm{mol} / \mathrm{L})$ & (mole fraction) \\
\hline 773.15 & 750.0000 & 52.759 & $2.6488 \mathrm{E}-03$
\end{tabular}

$\begin{array}{llll}773.15 & 1000.0000 & 56.060 & 3.0681 \mathrm{E}-03\end{array}$

$\begin{array}{llll}773.15 & 1000.0000 & 56.060 & 3.1046 \mathrm{E}-03\end{array}$

$\begin{array}{llll}773.15 & 1250.0000 & 58.730 & 3.2233 \mathrm{E}-03\end{array}$

$\begin{array}{llll}773.15 & 1500.0000 & 60.994 & 3.8817 \mathrm{E}-03\end{array}$

$\begin{array}{llll}773.15 & 1750.0000 & 62.971 & 4.1261 \mathrm{E}-03\end{array}$

$\begin{array}{llll}773.15 & 2000.0000 & 64.733 & 4.0742 \mathrm{E}-03\end{array}$

$773.15 \quad 2000.0000 \quad 64.733 \quad 4.1200 \mathrm{E}-03$

$873.15 \quad 500.0000 \quad 44.909 \quad 4.0894 \mathrm{E}-03$

$873.15 \quad 500.0000 \quad 44.909 \quad 3.8756 \mathrm{E}-03$

$873.15 \quad 500.0000 \quad 44.909 \quad 4.8241 \mathrm{E}-03$

$\begin{array}{llll}873.15 & 750.0000 & 49.952 & 6.1713 \mathrm{E}-03\end{array}$

$\begin{array}{llll}873.15 & 1000.0000 & 53.582 & 6.0880 \mathrm{E}-03\end{array}$

$\begin{array}{llll}873.15 & 1000.0000 & 53.582 & 6.4430 \mathrm{E}-03\end{array}$

$\begin{array}{llll}873.15 & 1000.0000 & 53.582 & 7.6076 \mathrm{E}-03\end{array}$

$873.15 \quad 1250.0000 \quad 56.470 \quad 7.3252 \mathrm{E}-03$

$\begin{array}{llll}873.15 & 1250.0000 & 56.470 & 7.1392 \mathrm{E}-03\end{array}$

$873.15 \quad 1500.0000 \quad 58.892 \quad 7.7412 \mathrm{E}-03$

$\begin{array}{llll}873.15 & 1750.0000 & 60.991 & 8.1705 \mathrm{E}-03\end{array}$

$873.15 \quad 2000.0000 \quad 62.851 \quad 8.7911 \mathrm{E}-03$

$888.15 \quad 750.0000 \quad 49.541 \quad 6.8666 \mathrm{E}-03$

$888.15 \quad 1000.0000 \quad 53.221 \quad 8.2203 \mathrm{E}-03$

$\begin{array}{llll}888.15 & 1250.0000 & 56.140 & 8.3668 \mathrm{E}-03\end{array}$

$\begin{array}{llll}888.15 & 1500.0000 & 58.585 & 9.1129 \mathrm{E}-03\end{array}$

$888.15 \quad 1750.0000 \quad 60.702 \quad 9.1536 \mathrm{E}-03$

$\begin{array}{llll}973.15 & 500.0000 & 41.655 & 7.6915 \mathrm{E}-03\end{array}$

$\begin{array}{llll}973.15 & 700.0000 & 46.326 & 9.8962 \mathrm{E}-03\end{array}$

$\begin{array}{llll}973.15 & 880.0000 & 49.465 & 1.1635 \mathrm{E}-02\end{array}$

$\begin{array}{llll}973.15 & 880.0000 & 49.465 & 1.1433 \mathrm{E}-02\end{array}$

$\begin{array}{llll}973.15 & 880.0000 & 49.465 & 1.1715 \mathrm{E}-02\end{array}$

$\begin{array}{llll}973.15 & 880.0000 & 49.465 & 1.1724 \mathrm{E}-02\end{array}$

$\begin{array}{llll}973.15 & 880.0000 & 49.465 & 1.0723 \mathrm{E}-02\end{array}$

$\begin{array}{llll}973.15 & 880.0000 & 49.465 & 1.2069 \mathrm{E}-02\end{array}$

$\begin{array}{llll}973.15 & 880.0000 & 49.465 & 1.0336 \mathrm{E}-02\end{array}$

$\begin{array}{llll}973.15 & 880.0000 & 49.465 & 1.1098 \mathrm{E}-02\end{array}$

$\begin{array}{llll}973.15 & 880.0000 & 49.465 & 1.0846 \mathrm{E}-02\end{array}$

$\begin{array}{llll}973.15 & 880.0000 & 49.465 & 1.1778 \mathrm{E}-02\end{array}$

$\begin{array}{llll}973.15 & 1200.0000 & 53.749 & 1.3484 \mathrm{E}-02\end{array}$

$973.15 \quad 1500.0000 \quad 56.894 \quad 1.4954 \mathrm{E}-02$

$973.15 \quad 1750.0000 \quad 59.110 \quad 1.8678 \mathrm{E}-02$

$1073.15 \quad 500.0000 \quad 38.609 \quad 1.1775 \mathrm{E}-02$

$1073.15 \quad 500.0000 \quad 38.609 \quad 1.1854 \mathrm{E}-02$

$1073.15 \quad 500.0000 \quad 38.609 \quad 1.1661 \mathrm{E}-02$

$1073.15 \quad 650.0000 \quad 42.606 \quad 1.6248 \mathrm{E}-02$

$\begin{array}{llll}1073.15 & 750.0000 & 44.743 & 1.8100 \mathrm{E}-02\end{array}$

$1073.15 \quad 750.0000 \quad 44.743 \quad 1.8355 \mathrm{E}-02$

$1073.15 \quad 1000.0000 \quad 48.991 \quad 2.1716 \mathrm{E}-02$

$\begin{array}{llll}1173.15 & 800.0000 & 43.393 & 3.1193 \mathrm{E}-02\end{array}$

$\begin{array}{llll}1173.15 & 800.0000 & 43.393 & 3.1072 \mathrm{E}-02\end{array}$

$\begin{array}{llll}1173.15 & 900.0000 & 45.245 & 3.7047 \mathrm{E}-02\end{array}$

\begin{tabular}{llll}
1173.15 & 1000.0000 & 46.891 & $4.1305 \mathrm{E}-02$ \\
\hline
\end{tabular} 
Compound: Silica (SiO2)

Reference: Heitmann, H.G., 1964, Solubility of silicic acid in water and steam and their influence on turbine

19 silication: Chemiker-Ztg./Chem. Apparatur, v. 88, n. 22 , p. 891-893

Units of original measurements: Temperature in degrees $C$, Pressure in $\mathrm{kgf} / \mathrm{cm} 2$, Solubility in $\mathrm{mg} / \mathrm{kg}$

Temperature Pressure Density Solubility

\begin{tabular}{llrr}
$(\mathrm{K})$ & $(\mathrm{MPa})$ & $(\mathrm{mol} / \mathrm{L})$ & (mole fraction) \\
\hline 368.15 & 0.0294 & 0.010 & $3.5980 \mathrm{E}-09$ \\
371.15 & 0.0490 & 0.016 & $4.4975 \mathrm{E}-09$ \\
393.15 & 0.0981 & 0.030 & $5.9967 \mathrm{E}-09$ \\
396.15 & 0.0490 & 0.015 & $5.0972 \mathrm{E}-09$ \\
420.15 & 0.2942 & 0.087 & $1.2293 \mathrm{E}-08$ \\
423.15 & 0.0294 & 0.008 & $4.4975 \mathrm{E}-09$ \\
424.15 & 0.0490 & 0.014 & $5.3970 \mathrm{E}-09$ \\
438.15 & 0.0981 & 0.027 & $7.1960 \mathrm{E}-09$ \\
453.15 & 0.2942 & 0.080 & $1.4692 \mathrm{E}-08$ \\
473.15 & 0.0490 & 0.012 & $7.7957 \mathrm{E}-09$ \\
473.15 & 0.8826 & 0.236 & $3.8978 \mathrm{E}-08$ \\
476.15 & 0.5884 & 0.153 & $2.5486 \mathrm{E}-08$ \\
478.15 & 0.0981 & 0.025 & $1.0194 \mathrm{E}-08$ \\
488.15 & 1.3729 & 0.363 & $8.0955 \mathrm{E}-08$ \\
493.15 & 0.0294 & 0.007 & $7.7957 \mathrm{E}-09$ \\
493.15 & 1.8633 & 0.502 & $1.2893 \mathrm{E}-07$ \\
506.15 & 0.2942 & 0.071 & $2.0689 \mathrm{E}-08$ \\
516.15 & 3.4323 & 0.948 & $3.5081 \mathrm{E}-07$ \\
521.15 & 0.8826 & 0.210 & $6.2965 \mathrm{E}-08$ \\
523.15 & 1.3729 & 0.332 & $1.0494 \mathrm{E}-07$ \\
523.15 & 1.8633 & 0.461 & $1.5591 \mathrm{E}-07$ \\
525.15 & 0.0981 & 0.023 & $1.4992 \mathrm{E}-08$ \\
527.15 & 0.5884 & 0.137 & $3.7779 \mathrm{E}-08$ \\
533.15 & 0.0490 & 0.011 & $1.1993 \mathrm{E}-08$ \\
538.15 & 4.9033 & 1.364 & $7.4958 \mathrm{E}-07$ \\
553.15 & 3.4323 & 0.837 & $4.5275 \mathrm{E}-07$ \\
563.15 & 0.2942 & 0.063 & $3.7479 \mathrm{E}-08$ \\
565.15 & 0.0981 & 0.021 & $2.1288 \mathrm{E}-08$ \\
572.15 & 0.5884 & 0.126 & $5.6968 \mathrm{E}-08$ \\
572.15 & 0.8826 & 0.190 & $1.1394 \mathrm{E}-07$ \\
573.15 & 0.0294 & 0.006 & $1.4992 \mathrm{E}-08$ \\
573.15 & 1.3729 & 0.298 & $1.7690 \mathrm{E}-07$ \\
573.15 & 1.8633 & 0.410 & $2.5186 \mathrm{E}-07$ \\
591.15 & 0.0490 & 0.010 & $2.0689 \mathrm{E}-08$ \\
603.15 & 0.2942 & 0.059 & $5.0972 \mathrm{E}-08$ \\
603.15 & 4.9033 & 1.098 & $1.1094 \mathrm{E}-06$ \\
604.15 & 3.4323 & 0.737 & $6.7463 \mathrm{E}-07$ \\
622.15 & 1.8633 & 0.373 & $4.4076 \mathrm{E}-07$ \\
623.15 & 0.5884 & 0.115 & $1.1094 \mathrm{E}-07$ \\
623.15 & 1.3729 & 0.272 & $3.2082 \mathrm{E}-07$ \\
626.15 & 0.0981 & 0.019 & $4.1377 \mathrm{E}-08$ \\
627.15 & 0.8826 & 0.172 & $2.2488 \mathrm{E}-07$ \\
638.15 & 0.0294 & 0.006 & $2.4886 \mathrm{E}-08$ \\
643.15 & 0.0981 & 0.018 & $5.0672 \mathrm{E}-08$
\end{tabular}

Temperature Pressure Density Solubility

\begin{tabular}{llll}
$(\mathrm{K})$ & $(\mathrm{MPa})$ & $(\mathrm{mol} / \mathrm{L})$ & $($ mole fraction $)$ \\
\hline 647.15 & 3.4323 & 0.675 & $1.0134 \mathrm{E}-06$ \\
648.15 & 4.9033 & 0.988 & $1.5891 \mathrm{E}-06$ \\
652.15 & 0.0490 & 0.009 & $3.3581 \mathrm{E}-08$ \\
662.15 & 0.2942 & 0.054 & $9.1449 \mathrm{E}-08$ \\
672.15 & 4.9033 & 0.942 & $2.0089 \mathrm{E}-06$ \\
673.15 & 0.5884 & 0.106 & $1.8590 \mathrm{E}-07$ \\
673.15 & 0.8826 & 0.160 & $3.4781 \mathrm{E}-07$ \\
673.15 & 1.3729 & 0.250 & $5.2471 \mathrm{E}-07$ \\
673.15 & 1.8633 & 0.341 & $7.2560 \mathrm{E}-07$ \\
678.15 & 3.4323 & 0.637 & $1.3912 \mathrm{E}-06$ \\
706.15 & 0.0981 & 0.017 & $8.4553 \mathrm{E}-08$ \\
713.15 & 0.0490 & 0.008 & $5.6968 \mathrm{E}-08$ \\
718.15 & 0.2942 & 0.049 & $1.5831 \mathrm{E}-07$ \\
722.15 & 0.8826 & 0.148 & $5.6968 \mathrm{E}-07$ \\
722.15 & 1.3729 & 0.232 & $8.7851 \mathrm{E}-07$ \\
723.15 & 0.5884 & 0.098 & $3.0583 \mathrm{E}-07$ \\
723.15 & 1.8633 & 0.316 & $1.3403 \mathrm{E}-06$ \\
723.15 & 3.4323 & 0.591 & $2.1438 \mathrm{E}-06$ \\
731.15 & 4.9033 & 0.847 & $3.7180 \mathrm{E}-06$ \\
758.15 & 0.0981 & 0.016 & $1.3133 \mathrm{E}-07$ \\
772.15 & 0.5884 & 0.092 & $4.8873 \mathrm{E}-07$ \\
773.15 & 1.8633 & 0.294 & $2.2488 \mathrm{E}-06$ \\
773.15 & 3.4323 & 0.548 & $3.9578 \mathrm{E}-06$ \\
774.15 & 1.3729 & 0.216 & $1.5112 \mathrm{E}-06$ \\
774.15 & 4.9033 & 0.792 & $5.7869 \mathrm{E}-06$ \\
776.15 & 0.8826 & 0.138 & $9.1749 \mathrm{E}-07$ \\
783.15 & 0.2942 & 0.045 & $3.1483 \mathrm{E}-07$ \\
799.15 & 4.9033 & 0.763 & $7.2561 \mathrm{E}-06$ \\
818.15 & 0.0981 & 0.014 & $1.9849 \mathrm{E}-07$ \\
823.15 & 0.5884 & 0.086 & $7.4359 \mathrm{E}-07$ \\
823.15 & 0.8826 & 0.130 & $1.3553 \mathrm{E}-06$ \\
823.15 & 1.3729 & 0.202 & $2.3327 \mathrm{E}-06$ \\
824.15 & 1.8633 & 0.275 & $3.5081 \mathrm{E}-06$ \\
824.15 & 3.4323 & 0.511 & $5.9368 \mathrm{E}-06$ \\
853.15 & 0.2942 & 0.042 & $4.6774 \mathrm{E}-07$ \\
860.15 & 0.0981 & 0.014 & $2.5636 \mathrm{E}-07$ \\
870.15 & 1.3729 & 0.191 & $3.0973 \mathrm{E}-06$ \\
870.15 & 4.9033 & 0.694 & $1.3103 \mathrm{E}-05$ \\
873.15 & 0.5884 & 0.081 & $9.6547 \mathrm{E}-07$ \\
873.15 & 0.8826 & 0.122 & $1.8110 \mathrm{E}-06$ \\
873.15 & 3.4323 & 0.480 & $8.6054 \mathrm{E}-06$ \\
874.15 & 1.8633 & 0.259 & $5.2471 \mathrm{E}-06$ \\
911.15 & 1.8633 & 0.248 & $6.3566 \mathrm{E}-06$ \\
913.15 & 3.4323 & 0.458 & $1.0614 \mathrm{E}-05$ \\
\hline & & &
\end{tabular}


Compound: Sodium Chloride (NaCl)

\begin{tabular}{|c|c|c|c|}
\hline \multicolumn{4}{|c|}{$\begin{array}{l}\text { Reference: Spillner, F., } 1940, \text { High pressure steam as a } \\
\text { solvent: Die Chemische Fabrik, v. 13, n. 22, p. } \\
\quad 01 \quad 405-416 \\
\text { Units of original measurements: Temperature in degrees C, } \\
\text { Pressure in kgf/cm2, Solubility in } \mathrm{mg} / \mathrm{kg} \text {, Data set excluded } \\
\text { from equation fit }\end{array}$} \\
\hline $\begin{array}{l}\text { Temperature } \\
(\mathrm{K})\end{array}$ & $\begin{array}{l}\text { Pressure } \\
(\mathrm{MPa})\end{array}$ & $\begin{array}{l}\text { Density } \\
(\mathrm{mol} / \mathrm{L})\end{array}$ & $\begin{array}{c}\text { Solubility } \\
\text { (mole fraction) }\end{array}$ \\
\hline 677.15 & 22.5553 & 6.828 & $1.2642 \mathrm{E}-04$ \\
\hline 678.15 & 14.7100 & 3.380 & $1.0173 \mathrm{E}-05$ \\
\hline 679.15 & 12.2583 & 2.652 & 5.5487E-06 \\
\hline 679.15 & 22.0650 & 6.418 & 1.1717E-04 \\
\hline 680.15 & 7.3550 & 1.446 & 2.4661E-06 \\
\hline 680.15 & 9.8066 & 2.014 & $3.0826 \mathrm{E}-06$ \\
\hline 680.15 & 9.8066 & 2.014 & 2.1578E-06 \\
\hline 680.15 & 26.9683 & 10.296 & 3.8257E-04 \\
\hline 681.15 & 16.6713 & 3.984 & 1.7571E-05 \\
\hline 681.15 & 19.6133 & 5.124 & 4.3160E-05 \\
\hline 681.15 & 24.5166 & 7.879 & $1.7578 \mathrm{E}-04$ \\
\hline
\end{tabular}

Reference: Galobardes, J.F., Van Hare, D.R. and Rogers

L.B., 1981, Solubility of sodium chloride in dry

02 steam: Journal of Chemical and Engineering Data, v. 26, p. 363-366

Units of original measurements: Temperature in degrees $C$, Pressure in $\mathrm{MPa}$, Solubility in $\mathrm{ppb}$ of $\mathrm{Na}$

\begin{tabular}{crrr}
$\begin{array}{c}\text { Temperature } \\
(\mathrm{K})\end{array}$ & $\begin{array}{c}\text { Pressure } \\
\text { (MPa) }\end{array}$ & $\begin{array}{c}\text { Density } \\
\text { (mol/L) }\end{array}$ & $\begin{array}{c}\text { Solubility } \\
\text { (mole fraction) }\end{array}$ \\
\hline 673.15 & 5.3900 & 1.041 & $6.4257 \mathrm{E}-08$ \\
673.15 & 6.2900 & 1.233 & $1.2224 \mathrm{E}-07$ \\
673.15 & 6.8900 & 1.365 & $1.6143 \mathrm{E}-07$ \\
673.15 & 7.2000 & 1.434 & $2.0531 \mathrm{E}-07$ \\
673.15 & 7.7600 & 1.561 & $2.2803 \mathrm{E}-07$ \\
673.15 & 8.6200 & 1.762 & $3.2990 \mathrm{E}-07$ \\
673.15 & 9.4800 & 1.970 & $4.8898 \mathrm{E}-07$ \\
673.15 & 10.3400 & 2.186 & $7.2093 \mathrm{E}-07$ \\
698.15 & 5.6100 & 1.036 & $8.7766 \mathrm{E}-08$ \\
698.15 & 6.5600 & 1.227 & $1.5046 \mathrm{E}-07$ \\
698.15 & 7.5200 & 1.427 & $1.9277 \mathrm{E}-07$ \\
723.15 & 1.3800 & 0.233 & $8.6198 \mathrm{E}-10$ \\
723.15 & 1.8100 & 0.307 & $9.4035 \mathrm{E}-10$ \\
723.15 & 2.2400 & 0.381 & $3.6047 \mathrm{E}-09$ \\
723.15 & 2.6700 & 0.456 & $5.0152 \mathrm{E}-09$ \\
723.15 & 3.1000 & 0.532 & $7.8362 \mathrm{E}-09$ \\
723.15 & 3.4500 & 0.595 & $1.4105 \mathrm{E}-08$ \\
723.15 & 4.3100 & 0.750 & $2.8210 \mathrm{E}-08$ \\
723.15 & 5.1700 & 0.908 & $5.4854 \mathrm{E}-08$ \\
723.15 & 5.8400 & 1.034 & $9.0900 \mathrm{E}-08$ \\
723.15 & 6.0300 & 1.070 & $8.7766 \mathrm{E}-08$ \\
723.15 & 6.8200 & 1.221 & $1.9042 \mathrm{E}-07$ \\
723.15 & 6.8900 & 1.235 & $1.5672 \mathrm{E}-07$ \\
723.15 & 7.7600 & 1.406 & $2.1550 \mathrm{E}-07$ \\
723.15 & 7.8200 & 1.417 & $2.5233 \mathrm{E}-07$
\end{tabular}

Temperature Pressure Density Solubility (K) $\quad(\mathrm{MPa}) \quad(\mathrm{mol} / \mathrm{L}) \quad($ mole fraction

\begin{tabular}{rrrr}
723.15 & 8.6200 & 1.578 & $3.7222 \mathrm{E}-07$ \\
723.15 & 9.4800 & 1.755 & $5.3286 \mathrm{E}-07$ \\
723.15 & 10.3400 & 1.936 & $7.6795 \mathrm{E}-07$ \\
748.15 & 6.0600 & 1.030 & $9.5602 \mathrm{E}-08$ \\
748.15 & 7.0900 & 1.218 & $1.8493 \mathrm{E}-07$ \\
748.15 & 8.1300 & 1.412 & $3.3774 \mathrm{E}-07$ \\
773.15 & 6.2800 & 1.028 & $1.5672 \mathrm{E}-07$ \\
773.15 & 6.8900 & 1.133 & $2.0531 \mathrm{E}-07$ \\
773.15 & 7.3600 & 1.216 & $2.5154 \mathrm{E}-07$ \\
773.15 & 7.7600 & 1.286 & $2.6800 \mathrm{E}-07$ \\
773.15 & 8.4400 & 1.408 & $3.3931 \mathrm{E}-07$ \\
773.15 & 8.6200 & 1.440 & $3.7849 \mathrm{E}-07$ \\
773.15 & 9.4800 & 1.596 & $5.8458 \mathrm{E}-07$ \\
773.15 & 10.3400 & 1.755 & $7.3269 \mathrm{E}-07$ \\
798.15 & 6.5000 & 1.025 & $1.9355 \mathrm{E}-07$ \\
798.15 & 7.6200 & 1.212 & $2.2882 \mathrm{E}-07$ \\
798.15 & 8.7500 & 1.404 & $3.4088 \mathrm{E}-07$ \\
823.15 & 6.7200 & 1.024 & $2.1315 \mathrm{E}-07$ \\
823.15 & 6.8900 & 1.051 & $2.2647 \mathrm{E}-07$ \\
823.15 & 7.7600 & 1.190 & $3.3696 \mathrm{E}-07$ \\
823.15 & 7.8900 & 1.211 & $3.3852 \mathrm{E}-07$ \\
823.15 & 8.6200 & 1.330 & $4.2237 \mathrm{E}-07$ \\
823.15 & 10.3400 & 1.614 & $8.7296 \mathrm{E}-07$ \\
\hline
\end{tabular}

Reference: Alekhin, Yu.V. and Vakulenko, A.G., 1988, Thermodynamic parameters and solubility of $04 \mathrm{NaCl}$ in water vapor at $300-500 \mathrm{C}$ up to $300 \mathrm{bar}$ : Geochemistry International, v. 25, n. 5, p. 97-110

Units of original measurements: Temperature in degrees $C$, Pressure in bar, Solubility in mole fraction, Data set excluded from equation fit

Temperature Pressure Density Solubility (K) $\quad(\mathrm{MPa}) \quad(\mathrm{mol} / \mathrm{L}) \quad$ (mole fraction)

$\begin{array}{rrrr}583.15 & 3.5740 & 0.809 & 1.3760 \mathrm{E}-06 \\ 583.15 & 4.7660 & 1.122 & 3.3800 \mathrm{E}-06 \\ 583.15 & 6.1550 & 1.527 & 7.0800 \mathrm{E}-06 \\ 583.15 & 6.7200 & 1.708 & 9.9080 \mathrm{E}-06 \\ 603.15 & 2.6290 & 0.555 & 1.1350 \mathrm{E}-06 \\ 603.15 & 4.9980 & 1.122 & 3.9700 \mathrm{E}-06 \\ 603.15 & 6.4950 & 1.527 & 8.0000 \mathrm{E}-06 \\ 603.15 & 7.7240 & 1.897 & 1.5700 \mathrm{E}-05 \\ 603.15 & 8.6800 & 2.215 & 2.3660 \mathrm{E}-05 \\ 623.15 & 3.8550 & 0.802 & 3.2600 \mathrm{E}-06 \\ 623.15 & 5.1850 & 1.113 & 4.7400 \mathrm{E}-06 \\ 623.15 & 6.7750 & 1.514 & 9.0800 \mathrm{E}-06 \\ 623.15 & 8.1000 & 1.881 & 1.8150 \mathrm{E}-05 \\ 623.15 & 10.6100 & 2.686 & 3.8550 \mathrm{E}-05 \\ 623.15 & 10.6500 & 2.700 & 3.8900 \mathrm{E}-05 \\ 673.15 & 2.9530 & 0.549 & 5.5400 \mathrm{E}-06 \\ 673.15 & 4.2250 & 0.801 & 7.2000 \mathrm{E}-06 \\ 673.15 & 5.7220 & 1.111 & 9.7200 \mathrm{E}-06 \\ 673.15 & 7.5450 & 1.512 & 1.4250 \mathrm{E}-05 \\ 673.15 & 9.1050 & 1.878 & 2.4310 \mathrm{E}-05\end{array}$




\begin{tabular}{crrr}
$\begin{array}{c}\text { Temperature } \\
(\mathrm{K})\end{array}$ & $\begin{array}{c}\text { Pressure } \\
\text { (MPa) }\end{array}$ & $\begin{array}{c}\text { Density } \\
\text { (mol/L) }\end{array}$ & $\begin{array}{c}\text { Solubility } \\
\text { (mole fraction) }\end{array}$ \\
\hline 673.15 & 12.1860 & 2.682 & $6.0000 \mathrm{E}-05$ \\
673.15 & 15.5000 & 3.711 & $1.2300 \mathrm{E}-04$ \\
673.15 & 17.2600 & 4.358 & $1.7300 \mathrm{E}-04$ \\
723.15 & 3.1920 & 0.549 & $1.0770 \mathrm{E}-05$ \\
723.15 & 4.5850 & 0.800 & $1.2740 \mathrm{E}-05$ \\
723.15 & 6.2390 & 1.110 & $1.6150 \mathrm{E}-05$ \\
723.15 & 8.2810 & 1.510 & $2.1230 \mathrm{E}-05$ \\
723.15 & 13.6500 & 2.678 & $8.3080 \mathrm{E}-05$ \\
723.15 & 17.6900 & 3.703 & $1.8500 \mathrm{E}-04$ \\
723.15 & 21.7700 & 4.916 & $3.4520 \mathrm{E}-04$ \\
723.15 & 25.1000 & 6.087 & $5.0700 \mathrm{E}-04$ \\
773.15 & 3.4280 & 0.548 & $2.0300 \mathrm{E}-05$ \\
773.15 & 4.9390 & 0.799 & $2.3600 \mathrm{E}-05$ \\
773.15 & 8.9940 & 1.508 & $3.8770 \mathrm{E}-05$ \\
773.15 & 10.9700 & 1.873 & $4.8190 \mathrm{E}-05$ \\
773.15 & 15.0400 & 2.673 & $1.2250 \mathrm{E}-04$ \\
773.15 & 19.7600 & 3.697 & $2.5020 \mathrm{E}-04$ \\
773.15 & 24.7400 & 4.914 & $5.0350 \mathrm{E}-04$ \\
773.15 & 32.3800 & 7.129 & $1.0450 \mathrm{E}-03$ \\
\hline
\end{tabular}

Reference: Armellini, F.J. and Tester, J.W., 1993, Solubility 05 of sodium chloride and sulfate in sub- and
supercritical water vapor from $450-550 \mathrm{C}$ and
$100-250$ bar: Fluid Phase Equilibria, v. $84, p$. $123-142$

Units of original measurements: Temperature in degrees $C$, Pressure in bar, Solubility in ppm

\begin{tabular}{cccc}
$\begin{array}{c}\text { Temperature } \\
(\mathrm{K})\end{array}$ & $\begin{array}{c}\text { Pressure } \\
(\mathrm{MPa})\end{array}$ & $\begin{array}{c}\text { Density } \\
(\mathrm{mol} / \mathrm{L})\end{array}$ & $\begin{array}{c}\text { Solubility } \\
\text { (mole fraction) }\end{array}$ \\
\hline 723.15 & 10.0000 & 1.864 & $4.6239 \mathrm{E}-07$ \\
723.15 & 15.0000 & 3.004 & $3.8532 \mathrm{E}-06$ \\
723.15 & 20.0000 & 4.363 & $1.9606 \mathrm{E}-05$ \\
773.15 & 10.0000 & 1.692 & $2.7743 \mathrm{E}-07$ \\
773.15 & 15.0000 & 2.665 & $4.2232 \mathrm{E}-06$ \\
773.15 & 20.0000 & 3.752 & $9.6795 \mathrm{E}-06$ \\
773.15 & 25.0000 & 4.982 & $3.1136 \mathrm{E}-05$ \\
823.15 & 10.0000 & 1.557 & $2.7743 \mathrm{E}-07$ \\
823.15 & 20.0000 & 3.350 & $1.0419 \mathrm{E}-05$ \\
823.15 & 25.0000 & 4.358 & $3.0211 \mathrm{E}-05$ \\
\hline
\end{tabular}

Reference: Sourirajan. S. and Kennedy, G.C., 1962, The 06 system $\mathrm{H} 2 \mathrm{O}-\mathrm{NaCl}$ at elevated temperatures and pressures: American Journal of Science, v. 260, p. 115-141

Units of original measurements: Temperature in degrees $C$, Pressure in bar, Solubility in wt\%, Data set excluded from equation fit

Temperature Pressure Density Solubility

$\begin{array}{llll}623.15 & 10.0000 & 2.474 & 6.1652 \mathrm{E}-06 \\ 623.15 & 11.4000 & 2.981 & 8.0148 \mathrm{E}-06 \\ 673.15 & 10.0000 & 2.100 & 1.2947 \mathrm{E}-05 \\ 673.15 & 15.0000 & 3.542 & 2.1271 \mathrm{E}-05 \\ 673.15 & 18.2000 & 4.743 & 2.4662 \mathrm{E}-05\end{array}$

Temperature Pressure Density Solubility

$\begin{array}{llll}723.15 & 10.0000 & 1.864 & 2.0654 \mathrm{E}-05\end{array}$

$723.15 \quad 15.0000 \quad 3.004 \quad 3.3603 E-05$

$\begin{array}{llll}723.15 & 20.0000 & 4.363 & 4.1310 \mathrm{E}-05\end{array}$

$723.15 \quad 25.0000 \quad 6.049 \quad 4.5935 \mathrm{E}-05$

$\begin{array}{llll}723.15 & 25.9000 & 6.399 & 4.8402 \mathrm{E}-05\end{array}$

$\begin{array}{llll}773.15 & 5.0000 & 0.809 & 6.7817 \mathrm{E}-06\end{array}$

$\begin{array}{llll}773.15 & 10.0000 & 1.692 & 2.7745 \mathrm{E}-05\end{array}$

$773.15 \quad 15.0000 \quad 2.665 \quad 4.3160 \mathrm{E}-05$

$773.15 \quad 20.0000 \quad 3.752 \quad 5.5493 \mathrm{E}-05$

$773.15 \quad 25.0000 \quad 4.982 \quad 6.4435 \mathrm{E}-05$

$\begin{array}{llll}773.15 & 30.0000 & 6.387 & 7.2144 \mathrm{E}-05\end{array}$

$\begin{array}{llll}773.15 & 33.0000 & 7.331 & 7.4919 E-05\end{array}$

$823.15 \quad 5.0000 \quad 0.753 \quad 1.1098 \mathrm{E}-05$

$823.15 \quad 10.0000 \quad 1.557 \quad 3.3603 \mathrm{E}-05$

$823.15 \quad 15.0000 \quad 2.419 \quad 5.0560 \mathrm{E}-05$

$823.15 \quad 20.0000 \quad 3.350 \quad 6.2585 \mathrm{E}-05$

$823.15 \quad 25.0000 \quad 4.358 \quad 7.3994 \mathrm{E}-05$

$823.15 \quad 30.0000 \quad 5.455 \quad 8.5095 \mathrm{E}-05$

$823.15 \quad 35.0000 \quad 6.649 \quad 9.6814 \mathrm{E}-05$

$823.15 \quad 37.9000 \quad 7.389 \quad 1.0576 \mathrm{E}-04$

$\begin{array}{llll}873.15 & 5.0000 & 0.705 & 1.9729 \mathrm{E}-05\end{array}$

$\begin{array}{llll}873.15 & 10.0000 & 1.446 & 3.8227 \mathrm{E}-05\end{array}$

$873.15 \quad 15.0000 \quad 2.227 \quad 5.6726 \mathrm{E}-05$

$\begin{array}{llll}873.15 & 20.0000 & 3.052 & 6.9369 \mathrm{E}-05\end{array}$

$\begin{array}{llll}873.15 & 25.0000 & 3.926 & 7.8928 \mathrm{E}-05\end{array}$

$873.15 \quad 30.0000 \quad 4.850 \quad 9.0338 \mathrm{E}-05$

$873.15 \quad 35.0000 \quad 5.829 \quad 1.0298 \mathrm{E}-04$

$\begin{array}{llll}873.15 & 38.0000 & 6.442 & 1.1624 \mathrm{E}-04\end{array}$

$873.15 \quad 39.2000 \quad 6.693 \quad 1.2765 \mathrm{E}-04$

$\begin{array}{llll}898.15 & 5.0000 & 0.684 & 2.8361 \mathrm{E}-05\end{array}$

$898.15 \quad 10.0000 \quad 1.398 \quad 4.4702 \mathrm{E}-05$

$898.15 \quad 15.0000 \quad 2.145 \quad 6.1660 \mathrm{E}-05$

$898.15 \quad 20.0000 \quad 2.929 \quad 7.8619 E-05$

$898.15 \quad 25.0000 \quad 3.751 \quad 9.8356 \mathrm{E}-05$

$898.15 \quad 30.0000 \quad 4.614 \quad 1.1779 \mathrm{E}-04$

$898.15 \quad 35.0000 \quad 5.519 \quad 1.4554 \mathrm{E}-04$

$\begin{array}{llll}898.15 & 37.9000 & 6.063 & 2.2946 \mathrm{E}-04\end{array}$

$\begin{array}{llll}923.15 & 2.0000 & 0.262 & 2.3120 \mathrm{E}-05\end{array}$

$923.15 \quad 5.0000 \quad 0.664 \quad 3.4527 \mathrm{E}-05$

$923.15 \quad 10.0000 \quad 1.353 \quad 5.4876 \mathrm{E}-05$

$923.15 \quad 15.0000 \quad 2.071 \quad 8.0161 \mathrm{E}-05$

$923.15 \quad 20.0000 \quad 2.818 \quad 1.1593 E-04$

$923.15 \quad 25.0000 \quad 3.597 \quad 1.6251 \mathrm{E}-04$

$923.15 \quad 30.0000 \quad 4.409 \quad 2.1959 E-04$

$923.15 \quad 35.6000 \quad 5.357 \quad 4.9376 \mathrm{E}-04$

$\begin{array}{llll}948.15 & 2.0000 & 0.255 & 3.7919 E-05\end{array}$

$\begin{array}{llll}948.15 & 5.0000 & 0.645 & 6.5668 \mathrm{E}-05\end{array}$

$948.15 \quad 10.0000 \quad 1.312 \quad 1.0853 E-04$

$948.15 \quad 15.0000 \quad 2.003 \quad 1.6961 \mathrm{E}-04$

$948.15 \quad 20.0000 \quad 2.718 \quad 2.5322 \mathrm{E}-04$

$948.15 \quad 25.0000 \quad 3.460 \quad 3.7022 \mathrm{E}-04$ 


\begin{tabular}{crrr}
$\begin{array}{c}\text { Temperature } \\
(\mathrm{K})\end{array}$ & $\begin{array}{c}\text { Pressure } \\
\text { (MPa) }\end{array}$ & $\begin{array}{c}\text { Density } \\
\text { (mol/L) }\end{array}$ & $\begin{array}{c}\text { Solubility } \\
\text { (mole fraction) }\end{array}$ \\
\hline 948.15 & 30.0000 & 4.228 & $6.6312 \mathrm{E}-04$ \\
948.15 & 32.6000 & 4.638 & $1.1791 \mathrm{E}-03$ \\
973.15 & 2.0000 & 0.249 & $8.0161 \mathrm{E}-05$ \\
973.15 & 5.0000 & 0.627 & $1.6374 \mathrm{E}-04$ \\
973.15 & 10.0000 & 1.273 & $3.1032 \mathrm{E}-04$ \\
973.15 & 15.0000 & 1.939 & $5.4165 \mathrm{E}-04$ \\
973.15 & 20.0000 & 2.627 & $9.2793 \mathrm{E}-04$ \\
973.15 & 25.2000 & 3.364 & $1.5733 \mathrm{E}-03$ \\
973.15 & 28.7000 & 3.874 & $2.9216 \mathrm{E}-03$ \\
998.15 & 2.0000 & 0.242 & $2.7946 \mathrm{E}-04$ \\
998.15 & 5.0000 & 0.610 & $6.3344 \mathrm{E}-04$ \\
998.15 & 10.0000 & 1.237 & $1.4253 \mathrm{E}-03$ \\
998.15 & 15.0000 & 1.881 & $2.7945 \mathrm{E}-03$ \\
998.15 & 20.0000 & 2.542 & $5.6929 \mathrm{E}-03$ \\
1023.15 & 2.0000 & 0.236 & $3.9872 \mathrm{E}-03$ \\
1023.15 & 5.0000 & 0.595 & $6.7435 \mathrm{E}-03$ \\
1023.15 & 7.5000 & 0.897 & $1.0107 \mathrm{E}-02$ \\
1023.15 & 10.0000 & 1.203 & $1.4403 \mathrm{E}-02$ \\
1023.15 & 13.0000 & 1.575 & $2.2727 \mathrm{E}-02$ \\
\hline
\end{tabular}

Reference: Olander, A. and Liander, H., 1950, The phase 07 diagram of sodium chloride and steam above the 07 critical point: Acta Chemica Scandinaviga, v. 4, p. $1437-1445$

Units of original measurements: Temperature in degrees $C$, Pressure in $\mathrm{kgf} / \mathrm{cm} 2$, Solubility in $w t \%$

Temperature Pressure Density Solubility

\begin{tabular}{clrc}
$(\mathrm{K})$ & $(\mathrm{MPa})$ & $(\mathrm{mol} / \mathrm{L})$ & $($ mole fraction $)$ \\
\hline 653.15 & 15.2003 & 3.966 & $1.8496 \mathrm{E}-05$ \\
663.15 & 16.5732 & 4.310 & $2.4662 \mathrm{E}-05$ \\
673.15 & 17.9462 & 4.636 & $3.0828 \mathrm{E}-05$ \\
683.15 & 19.4172 & 4.984 & $4.0077 \mathrm{E}-05$ \\
693.15 & 20.8882 & 5.311 & $4.9327 \mathrm{E}-05$ \\
703.15 & 22.2611 & 5.576 & $6.1660 \mathrm{E}-05$ \\
713.15 & 23.8302 & 5.901 & $8.0161 \mathrm{E}-05$ \\
723.15 & 25.3012 & 6.164 & $1.0175 \mathrm{E}-04$ \\
733.15 & 26.7722 & 6.409 & $1.2025 \mathrm{E}-04$ \\
748.15 & 29.1258 & 6.794 & $1.5727 \mathrm{E}-04$ \\
\hline
\end{tabular}

Reference: Styrikovich, M.A., Khaibullin, I.Kh. and 08 high pressure steam: Dok. Akad. Nauk SSSR, v. 100, p. $1123-1126$

Units of original measurements: Temperature in degrees $C$, Pressure in atm, Solubility in mg/kg, Original data in graphical format

\begin{tabular}{crrc}
$\begin{array}{c}\text { Temperature } \\
(\mathrm{K})\end{array}$ & $\begin{array}{c}\text { Pressure } \\
(\mathrm{MPa})\end{array}$ & $\begin{array}{c}\text { Density } \\
(\mathrm{mol} / \mathrm{L})\end{array}$ & $\begin{array}{c}\text { Solubility } \\
\text { (mole fraction) }\end{array}$ \\
\hline 673.15 & 3.0398 & 0.566 & $1.3133 \mathrm{E}-07$ \\
673.30 & 10.1325 & 2.132 & $1.4447 \mathrm{E}-06$ \\
673.45 & 14.1855 & 3.274 & $6.0226 \mathrm{E}-06$ \\
673.60 & 14.1855 & 3.272 & $5.5536 \mathrm{E}-06$ \\
674.81 & 3.0398 & 0.565 & $3.0957 \mathrm{E}-07$ \\
674.96 & 3.0398 & 0.565 & $3.9400 \mathrm{E}-07$
\end{tabular}

Temperature Pressure Density Solubility

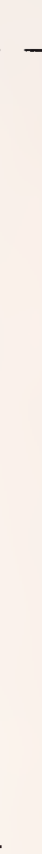

(K)

694.86

696.07

696.82

696.82

697.57

699.23

701.49

703.45

703.60

720.79

720.94

721.69

723.20

723.80

724.26

724.41

724.86

725.16

725.31

725.91

726.07

726.22

726.67

745.21

746.42

747.02

748.68

766.32

766.77

768.73

769.33

769.48

770.54

771.44

771.74

772.04

772.35

772.35

773.25

791.19

791.19

817.57

818.93

820.29

820.44

820.44

820.74

821.19

821.49

822.85

823.00
(MPa)

$(\mathrm{mol} / \mathrm{L})$

(mole fraction)

$\begin{array}{lll}15.4014 & 3.387 & 5.0376 \mathrm{E}-06\end{array}$

15.4014

3.373

$5.8256 \mathrm{E}-06$

15.4014

$3.364 \quad 5.0939 \mathrm{E}-06$

18.2385

18.2385

4.245

1.3143E-05

18.2385

14.1855

14.1855

14.1855

18.2385

14.1855

18.2385

3.0398

18.2385

14.1855

15.4014

18.2385

10.1325

3.0398

14.1855

18.2385

3.0398

15.4014

18.2385

14.1855

18.2385

14.1855

14.1855

18.2385

18.2385

3.0398

14.1855

18.2385

10.1325

18.2385

3.0398

14.1855

18.2385

14.1855

14.1855

18.2385

18.2385

3.0398

18.2385

10.1325

14.1855

10.1325

14.1855

18.2385

3.0398

18.2385
$4.232 \quad 1.5066 \mathrm{E}-05$

4.203 1.3106E-05

$2.981 \quad 3.4616 \mathrm{E}-06$

2.963 3.7805E-06

$2.962 \quad 3.3302 E-06$

$3.884 \quad 9.1372 \mathrm{E}-06$

$2.822 \quad 2.8612 \mathrm{E}-06$

$3.873 \quad 8.8745 \mathrm{E}-06$

$0.522 \quad 5.9100 \mathrm{E}-07$

$3.846 \quad 8.9965 \mathrm{E}-06$

$2.797 \quad 2.7674 \mathrm{E}-06$

3.093 4.0057E-06

$3.833 \quad 9.0809 \mathrm{E}-06$

$1.884 \quad 9.8500 \mathrm{E}-07$

$0.520 \quad 6.5666 \mathrm{E}-07$

$2.786 \quad 2.9925 \mathrm{E}-06$

$3.818 \quad 8.9777 \mathrm{E}-06$

$0.519 \quad 4.1276 \mathrm{E}-07$

$3.074 \quad 4.0244 \mathrm{E}-06$

$3.606 \quad 7.5142 \mathrm{E}-06$

$2.650 \quad 2.5235 \mathrm{E}-06$

$3.588 \quad 7.5518 \mathrm{E}-06$

$2.636 \quad 2.5141 \mathrm{E}-06$

$2.535 \quad 2.5516 \mathrm{E}-06$

$3.407 \quad 7.2140 \mathrm{E}-06$

$3.391 \quad 7.2328 \mathrm{E}-06$

$0.487 \quad 4.4090 \mathrm{E}-07$

$2.519 \quad 2.5516 \mathrm{E}-06$

$3.376 \quad 7.2516 \mathrm{E}-06$

$1.722 \quad 1.2946 \mathrm{E}-06$

$3.366 \quad 6.9983 \mathrm{E}-06$

$0.485 \quad 9.5685 \mathrm{E}-07$

$2.504 \quad 2.8331 \mathrm{E}-06$

$3.361 \quad 7.0921 \mathrm{E}-06$

2.499 2.7299E-06

$2.411 \quad 2.8893 \mathrm{E}-06$

$3.219 \quad 7.1296 \mathrm{E}-06$

$3.047 \quad 7.6362 \mathrm{E}-06$

$0.455 \quad 2.2420 \mathrm{E}-06$

$3.030 \quad 7.6174 \mathrm{E}-06$

$1.586 \quad 1.8011 \mathrm{E}-06$

$2.285 \quad 3.9869 E-06$

$1.585 \quad 2.7768 \mathrm{E}-06$

$2.282 \quad 3.8181 \mathrm{E}-06$

$3.023 \quad 7.5049 \mathrm{E}-06$

$0.453 \quad 1.8199 \mathrm{E}-06$

$3.014 \quad 7.5518 \mathrm{E}-06$ 


\begin{tabular}{cccc}
$\begin{array}{c}\text { Temperature } \\
(\mathrm{K})\end{array}$ & $\begin{array}{c}\text { Pressure } \\
\text { (MPa) }\end{array}$ & $\begin{array}{c}\text { Density } \\
\text { (mol/L) }\end{array}$ & $\begin{array}{c}\text { Solubility } \\
\text { (mole fraction) }\end{array}$ \\
\hline 823.15 & 18.2385 & 3.014 & $7.6831 \mathrm{E}-06$ \\
824.05 & 10.1325 & 1.577 & $2.3452 \mathrm{E}-06$ \\
824.36 & 10.1325 & 1.576 & $2.0169 \mathrm{E}-06$ \\
824.36 & 10.1325 & 1.576 & $2.5329 \mathrm{E}-06$ \\
824.51 & 14.1855 & 2.269 & $3.8274 \mathrm{E}-06$ \\
826.01 & 3.0398 & 0.451 & $2.5235 \mathrm{E}-06$ \\
826.01 & 3.0398 & 0.451 & $2.3734 \mathrm{E}-06$ \\
\hline
\end{tabular}

Reference: Aleinikov, G.I., et. al., 1956, Solubility of sodium 09 Thloride in a superheated vapor ...:

Units of original measurements: Temperature in degrees $C$, Pressure in $\mathrm{kgf} / \mathrm{cm} 2$, Solubility in $\mathrm{mg} / \mathrm{kg}$, Original data in graphical format

\begin{tabular}{cccc}
$\begin{array}{c}\text { Temperature } \\
(\mathrm{K})\end{array}$ & $\begin{array}{c}\text { Pressure } \\
\text { (MPa) }\end{array}$ & $\begin{array}{c}\text { Density } \\
\text { (mol/L) }\end{array}$ & $\begin{array}{c}\text { Solubility } \\
\text { (mole fraction) }\end{array}$ \\
\hline 633.15 & 10.7873 & 2.635 & $2.5653 \mathrm{E}-06$ \\
639.20 & 10.7873 & 2.573 & $2.6498 \mathrm{E}-06$ \\
641.31 & 10.7873 & 2.553 & $2.8714 \mathrm{E}-06$ \\
642.23 & 10.7873 & 2.544 & $2.4808 \mathrm{E}-06$ \\
644.19 & 10.7873 & 2.526 & $2.1958 \mathrm{E}-06$ \\
644.19 & 10.7873 & 2.526 & $1.7524 \mathrm{E}-06$ \\
648.27 & 10.7873 & 2.490 & $2.0691 \mathrm{E}-06$ \\
649.40 & 10.7873 & 2.480 & $2.4175 \mathrm{E}-06$ \\
650.46 & 10.7873 & 2.471 & $1.4991 \mathrm{E}-06$ \\
650.46 & 10.7873 & 2.471 & $2.0163 \mathrm{E}-06$ \\
653.48 & 10.7873 & 2.446 & $1.6469 \mathrm{E}-06$ \\
653.48 & 10.7873 & 2.446 & $1.5307 \mathrm{E}-06$ \\
653.55 & 10.7873 & 2.446 & $1.7947 \mathrm{E}-06$ \\
655.52 & 10.7873 & 2.430 & $1.8580 \mathrm{E}-06$ \\
655.52 & 10.7873 & 2.430 & $1.3935 \mathrm{E}-06$ \\
655.59 & 10.7873 & 2.429 & $1.4991 \mathrm{E}-06$ \\
659.46 & 10.7873 & 2.399 & $1.3618 \mathrm{E}-06$ \\
659.53 & 10.7873 & 2.399 & $1.7208 \mathrm{E}-06$ \\
660.65 & 10.7873 & 2.390 & $1.4252 \mathrm{E}-06$ \\
661.64 & 10.7873 & 2.383 & $1.5096 \mathrm{E}-06$ \\
662.55 & 10.7873 & 2.376 & $1.3196 \mathrm{E}-06$ \\
662.70 & 10.7873 & 2.375 & $1.6363 \mathrm{E}-06$ \\
663.61 & 10.7873 & 2.368 & $1.3513 \mathrm{E}-06$ \\
663.68 & 10.7873 & 2.368 & $1.4674 \mathrm{E}-06$ \\
664.66 & 10.7873 & 2.361 & $1.3407 \mathrm{E}-06$ \\
664.74 & 10.7873 & 2.360 & $1.4991 \mathrm{E}-06$ \\
665.65 & 10.7873 & 2.354 & $1.3724 \mathrm{E}-06$ \\
665.79 & 10.7873 & 2.353 & $1.4674 \mathrm{E}-06$ \\
666.71 & 10.7873 & 2.346 & $1.6257 \mathrm{E}-06$ \\
668.89 & 10.7873 & 2.331 & $1.1296 \mathrm{E}-06$ \\
671.84 & 10.7873 & 2.311 & $1.5096 \mathrm{E}-06$ \\
674.94 & 10.7873 & 2.291 & $1.2035 \mathrm{E}-06$ \\
675.92 & 10.7873 & 2.284 & $1.0346 \mathrm{E}-06$ \\
678.95 & 10.7873 & 2.265 & $1.0557 \mathrm{E}-06$ \\
680.00 & 10.7873 & 2.258 & $9.9234 \mathrm{E}-07$ \\
681.06 & 10.7873 & 2.252 & $1.1718 \mathrm{E}-06$
\end{tabular}

Temperature Pressure Density Solubility

\begin{tabular}{llrc}
$(\mathrm{K})$ & $(\mathrm{MPa})$ & $(\mathrm{mol} / \mathrm{L})$ & $($ mole fraction $)$ \\
\hline 686.12 & 10.7873 & 2.221 & $1.1929 \mathrm{E}-06$ \\
687.18 & 10.7873 & 2.215 & $9.0788 \mathrm{E}-07$ \\
687.25 & 10.7873 & 2.215 & $1.1718 \mathrm{E}-06$ \\
690.48 & 10.7873 & 2.196 & $1.2563 \mathrm{E}-06$ \\
691.40 & 10.7873 & 2.191 & $8.2343 \mathrm{E}-07$ \\
692.31 & 10.7873 & 2.186 & $9.0788 \mathrm{E}-07$ \\
692.45 & 10.7873 & 2.185 & $1.2351 \mathrm{E}-06$ \\
692.87 & 10.7873 & 2.183 & $8.9732 \mathrm{E}-07$ \\
694.28 & 10.7873 & 2.175 & $9.5011 \mathrm{E}-07$ \\
694.35 & 10.7873 & 2.175 & $8.5510 \mathrm{E}-07$ \\
695.48 & 10.7873 & 2.168 & $9.0788 \mathrm{E}-07$ \\
\hline
\end{tabular}

Reference: Styrikovich, M.A., 1957, Solubility of salts in steam of supercritical characteristics:

10 Teploenergetika, v. 4, n. 2, p. 3-7

Units of original measurements: Temperature in degrees $C$, Pressure in $\mathrm{kg} / \mathrm{cm} 2$, Solubility in $\mathrm{mg} / \mathrm{kg}$, Original data in graphical format

Temperature Pressure Density Solubility

\begin{tabular}{llrr}
$(\mathrm{K})$ & $(\mathrm{MPa})$ & $(\mathrm{mol} / \mathrm{L})$ & (mole fraction) \\
\hline 661.47 & 21.5746 & 7.314 & $6.3524 \mathrm{E}-05$
\end{tabular}

$\begin{array}{llll}673.95 & 23.5360 & 7.770 & 8.4442 \mathrm{E}-05\end{array}$

$\begin{array}{llll}693.29 & 21.5746 & 5.596 & 3.4444 \mathrm{E}-05\end{array}$

$694.01 \quad 21.5746 \quad 5.573 \quad 3.6233 E-05$

$\begin{array}{llll}694.55 & 23.5360 & 6.459 & 5.9162 \mathrm{E}-05\end{array}$

$\begin{array}{llll}695.46 & 25.4973 & 7.472 & 9.9544 \mathrm{E}-05\end{array}$

$\begin{array}{llll}697.45 & 23.5360 & 6.334 & 5.6142 \mathrm{E}-05\end{array}$

$\begin{array}{llll}699.25 & 27.4586 & 8.419 & 1.7138 \mathrm{E}-04\end{array}$

$\begin{array}{llll}701.24 & 25.4973 & 7.139 & 8.4106 \mathrm{E}-05\end{array}$

$\begin{array}{llll}702.14 & 17.6520 & 3.969 & 1.2524 \mathrm{E}-05\end{array}$

$\begin{array}{llll}703.41 & 25.4973 & 7.027 & 8.4330 \mathrm{E}-05\end{array}$

$\begin{array}{llll}707.93 & 17.6520 & 3.886 & 1.0959 \mathrm{E}-05\end{array}$

$\begin{array}{llll}714.44 & 25.4973 & 6.546 & 5.4688 \mathrm{E}-05\end{array}$

$\begin{array}{llll}721.67 & 19.1230 & 4.126 & 1.3755 \mathrm{E}-05\end{array}$

$\begin{array}{llll}722.57 & 23.5360 & 5.528 & 3.6345 \mathrm{E}-05\end{array}$

$\begin{array}{llll}724.02 & 19.1230 & 4.093 & 1.3755 E-05\end{array}$

$\begin{array}{llll}726.01 & 19.1230 & 4.066 & 1.4873 E-05\end{array}$

$\begin{array}{llll}726.19 & 19.1230 & 4.063 & 1.1742 \mathrm{E}-05\end{array}$

$\begin{array}{llll}726.91 & 25.4973 & 6.124 & 5.6142 \mathrm{E}-05\end{array}$

$\begin{array}{llll}728.36 & 23.5360 & 5.388 & 3.4108 \mathrm{E}-05\end{array}$

$\begin{array}{llll}730.52 & 25.4973 & 6.018 & 5.1220 \mathrm{E}-05\end{array}$

$\begin{array}{llll}731.97 & 23.5360 & 5.307 & 2.9970 \mathrm{E}-05\end{array}$

$\begin{array}{llll}731.97 & 27.4586 & 6.711 & 8.6679 \mathrm{E}-05\end{array}$

$\begin{array}{llll}734.86 & 21.5746 & 4.643 & 1.8563 \mathrm{E}-05\end{array}$

$\begin{array}{llll}747.52 & 23.5360 & 5.001 & 2.5497 \mathrm{E}-05\end{array}$

$\begin{array}{llll}750.23 & 27.4586 & 6.161 & 5.7148 \mathrm{E}-05\end{array}$

$\begin{array}{llll}750.41 & 29.4200 & 6.827 & 7.7394 \mathrm{E}-05\end{array}$

$\begin{array}{llll}753.84 & 27.4586 & 6.070 & 5.2786 \mathrm{E}-05\end{array}$

$\begin{array}{llll}754.02 & 29.4200 & 6.716 & 7.7394 \mathrm{E}-05\end{array}$

$\begin{array}{llll}757.28 & 25.4973 & 5.394 & 3.6010 \mathrm{E}-05\end{array}$

$\begin{array}{llll}759.63 & 21.5746 & 4.284 & 1.5656 \mathrm{E}-05\end{array}$

$\begin{array}{llll}765.59 & 21.5746 & 4.210 & 1.5208 \mathrm{E}-05\end{array}$ 


\begin{tabular}{cccc}
$\begin{array}{c}\text { Temperature } \\
\text { (K) }\end{array}$ & $\begin{array}{c}\text { Pressure } \\
\text { (MPa) }\end{array}$ & $\begin{array}{c}\text { Density } \\
\text { (mol/L) }\end{array}$ & $\begin{array}{c}\text { Solubility } \\
\text { (mole fraction) }\end{array}$ \\
\hline 773.55 & 17.6520 & 3.223 & $7.1567 \mathrm{E}-06$ \\
774.27 & 23.5360 & 4.590 & $2.1695 \mathrm{E}-05$ \\
780.05 & 27.4586 & 5.520 & $4.7753 \mathrm{E}-05$ \\
780.78 & 29.4200 & 6.046 & $6.5537 \mathrm{E}-05$ \\
783.13 & 21.5746 & 4.015 & $1.5208 \mathrm{E}-05$ \\
783.13 & 27.4586 & 5.465 & $4.2497 \mathrm{E}-05$ \\
784.94 & 29.4200 & 5.961 & $6.1063 \mathrm{E}-05$ \\
786.02 & 19.1230 & 3.444 & $1.1183 \mathrm{E}-05$ \\
787.47 & 21.5746 & 3.971 & $1.4537 \mathrm{E}-05$ \\
787.47 & 29.4200 & 5.910 & $6.4531 \mathrm{E}-05$ \\
788.37 & 27.4586 & 5.377 & $4.5404 \mathrm{E}-05$ \\
788.91 & 25.4973 & 4.876 & $3.0977 \mathrm{E}-05$ \\
791.08 & 25.4973 & 4.847 & $2.9635 \mathrm{E}-05$ \\
794.15 & 23.5360 & 4.346 & $2.2589 \mathrm{E}-05$ \\
798.67 & 23.5360 & 4.297 & $2.2589 \mathrm{E}-05$ \\
800.48 & 19.1230 & 3.334 & $1.1183 \mathrm{E}-05$ \\
805.90 & 23.5360 & 4.220 & $2.2701 \mathrm{E}-05$ \\
807.71 & 25.4973 & 4.636 & $2.7174 \mathrm{E}-05$ \\
808.62 & 23.5360 & 4.193 & $2.2701 \mathrm{E}-05$ \\
810.42 & 17.6520 & 2.977 & $7.3804 \mathrm{E}-06$ \\
815.12 & 21.5746 & 3.722 & $1.7333 \mathrm{E}-05$ \\
820.91 & 29.4200 & 5.355 & $5.0773 \mathrm{E}-05$ \\
822.90 & 29.4200 & 5.327 & $5.0773 \mathrm{E}-05$ \\
826.87 & 27.4586 & 4.841 & $3.6793 \mathrm{E}-05$ \\
827.96 & 25.4973 & 4.414 & $2.5497 \mathrm{E}-05$ \\
829.22 & 27.4586 & 4.814 & $3.6793 \mathrm{E}-05$ \\
830.13 & 25.4973 & 4.392 & $2.7845 \mathrm{E}-05$ \\
834.47 & 19.1230 & 3.112 & $1.1406 \mathrm{E}-05$ \\
834.83 & 21.5746 & 3.571 & $1.6774 \mathrm{E}-05$ \\
836.63 & 23.5360 & 3.937 & $2.3484 \mathrm{E}-05$ \\
839.35 & 17.6520 & 2.818 & $7.9395 \mathrm{E}-06$ \\
843.14 & 27.4586 & 4.660 & $4.0819 \mathrm{E}-05$ \\
843.87 & 21.5746 & 3.508 & $1.8004 \mathrm{E}-05$ \\
843.87 & 29.4200 & 5.056 & $5.3457 \mathrm{E}-05$ \\
845.67 & 27.4586 & 4.634 & $4.0931 \mathrm{E}-05$ \\
847.30 & 23.5360 & 3.852 & $2.4714 \mathrm{E}-05$ \\
848.38 & 19.1230 & 3.033 & $1.4202 \mathrm{E}-05$ \\
848.57 & 29.4200 & 5.001 & $5.3793 \mathrm{E}-05$ \\
852.36 & 25.4973 & 4.185 & $3.1760 \mathrm{E}-05$ \\
\hline & & & \\
\hline
\end{tabular}

Reference: Sastry, V., 1957, Die Loslichkeit von Salzen in Hochgespanntem Wasserdampf und die Klarung der Frage der Turbinenversalzung: Doctoral Dissertation - Technische Hochschule Karlsruhe

Units of original measurements: Temperature in degrees $C$, Pressure in $\mathrm{kgf/} / \mathrm{cm} 2$, Solubility in $\mathrm{mg} / \mathrm{kg}$

Temperature Pressure Density Solubility

\begin{tabular}{lrrr}
$(\mathrm{K})$ & $(\mathrm{MPa})$ & $(\mathrm{mol} / \mathrm{L})$ & (mole fraction) \\
\hline 637.15 & 8.2376 & 1.839 & $4.2848 \mathrm{E}-07$ \\
637.15 & 8.9240 & 2.029 & $6.1651 \mathrm{E}-07$ \\
637.15 & 9.3163 & 2.142 & $7.7064 \mathrm{E}-07$ \\
637.15 & 9.7086 & 2.258 & $1.1097 \mathrm{E}-06$
\end{tabular}

Temperature Pressure Density Solubility

\begin{tabular}{rrrr}
\multicolumn{1}{c}{ (K) } & \multicolumn{1}{c}{ (MPa) } & $(\mathrm{mol} / \mathrm{L})$ & (mole fraction) \\
\hline 637.15 & 9.9047 & 2.317 & $8.6312 \mathrm{E}-07$ \\
637.15 & 10.1008 & 2.377 & $8.9395 \mathrm{E}-07$ \\
637.15 & 10.4931 & 2.499 & $7.0899 \mathrm{E}-07$ \\
637.15 & 10.7873 & 2.593 & $1.4180 \mathrm{E}-06$ \\
637.15 & 10.9834 & 2.658 & $9.8642 \mathrm{E}-07$ \\
637.15 & 11.3757 & 2.789 & $9.2477 \mathrm{E}-07$ \\
637.15 & 11.5719 & 2.856 & $2.0653 \mathrm{E}-06$ \\
646.15 & 6.0801 & 1.260 & $4.7472 \mathrm{E}-07$ \\
646.15 & 7.5511 & 1.617 & $2.9593 \mathrm{E}-07$ \\
646.15 & 7.6492 & 1.641 & $4.0690 \mathrm{E}-07$ \\
646.15 & 8.5318 & 1.869 & $5.1171 \mathrm{E}-07$ \\
646.15 & 9.0221 & 2.001 & $5.1171 \mathrm{E}-07$ \\
646.15 & 9.4144 & 2.109 & $4.6855 \mathrm{E}-07$ \\
646.15 & 9.5124 & 2.136 & $7.7064 \mathrm{E}-07$ \\
646.15 & 10.0028 & 2.276 & $7.3982 \mathrm{E}-07$ \\
646.15 & 10.2970 & 2.362 & $6.1651 \mathrm{E}-07$ \\
646.15 & 10.3950 & 2.391 & $1.1097 \mathrm{E}-06$ \\
646.15 & 10.9834 & 2.569 & $1.4796 \mathrm{E}-06$ \\
646.15 & 11.2777 & 2.660 & $1.1406 \mathrm{E}-06$ \\
646.15 & 11.7680 & 2.818 & $1.5721 \mathrm{E}-06$ \\
646.15 & 11.8661 & 2.850 & $1.9112 \mathrm{E}-06$ \\
646.15 & 11.9641 & 2.883 & $2.2503 \mathrm{E}-06$ \\
646.15 & 12.5525 & 3.083 & $2.7743 \mathrm{E}-06$ \\
646.15 & 12.5525 & 3.083 & $2.0962 \mathrm{E}-06$ \\
646.15 & 12.7487 & 3.152 & $2.4969 \mathrm{E}-06$ \\
646.15 & 13.3370 & 3.365 & $4.3156 \mathrm{E}-06$ \\
646.15 & 13.3370 & 3.365 & $3.0518 \mathrm{E}-06$ \\
675.15 & 3.5304 & 0.660 & $4.0073 \mathrm{E}-07$ \\
675.15 & 4.7072 & 0.896 & $2.1578 \mathrm{E}-07$ \\
675.15 & 5.8840 & 1.141 & $3.6991 \mathrm{E}-07$ \\
675.15 & 6.9627 & 1.375 & $3.0826 \mathrm{E}-07$ \\
675.15 & 8.0414 & 1.618 & $5.5486 \mathrm{E}-07$ \\
675.15 & 8.6298 & 1.755 & $4.9321 \mathrm{E}-07$ \\
675.15 & 8.7279 & 1.779 & $3.6991 \mathrm{E}-07$ \\
675.15 & 9.6105 & 1.992 & $4.6239 \mathrm{E}-07$ \\
675.15 & 9.6105 & 1.992 & $5.8569 \mathrm{E}-07$ \\
675.15 & 10.1008 & 2.114 & $1.2947 \mathrm{E}-06$ \\
675.15 & 10.1989 & 2.138 & $9.2477 \mathrm{E}-07$ \\
675.15 & 10.2970 & 2.163 & $7.0899 \mathrm{E}-07$ \\
675.15 & 10.4931 & 2.213 & $5.5486 \mathrm{E}-07$ \\
675.15 & 10.8854 & 2.315 & $9.5560 \mathrm{E}-07$ \\
675.15 & 11.0815 & 2.366 & $9.2477 \mathrm{E}-07$ \\
675.15 & 11.0815 & 2.366 & $7.3982 \mathrm{E}-07$ \\
675.15 & 11.2777 & 2.418 & $1.2947 \mathrm{E}-06$ \\
675.15 & 11.7680 & 2.550 & $1.1714 \mathrm{E}-06$ \\
675.15 & 12.0622 & 2.631 & $1.1097 \mathrm{E}-06$ \\
675.15 & 12.1603 & 2.658 & $1.8495 \mathrm{E}-06$ \\
& 12.2583 & 2.685 & $2.1578 \mathrm{E}-06$ \\
675.15 & 12.3564 & 2.713 & $1.8495 \mathrm{E}-06$ \\
6 & & &
\end{tabular}


Temperature Pressure Density Solubility Temperature Pressure Density Solubility

\begin{tabular}{|c|c|c|c|c|c|c|c|}
\hline$(\mathrm{K})$ & $(\mathrm{MPa})$ & $(\mathrm{mol} / \mathrm{L})$ & (mole fraction) & $(\mathrm{K})$ & (MPa) & $(\mathrm{mol} / \mathrm{L})$ & (mole fraction) \\
\hline 675.15 & 13.1409 & 2.938 & $1.9112 E-06$ & 759.15 & 16.0829 & 2.984 & $4.4081 E-06$ \\
\hline 675.15 & 13.2390 & 2.967 & 2.4661E-06 & 759.15 & 16.2790 & 3.027 & 5.0863E-06 \\
\hline 675.15 & 13.4351 & 3.025 & 2.1578E-06 & 759.15 & 16.9655 & 3.181 & 5.3329E-06 \\
\hline 675.15 & 13.4351 & 3.025 & $3.0826 \mathrm{E}-06$ & 759.15 & 17.1616 & 3.225 & 5.7336E-06 \\
\hline 675.15 & 13.7293 & 3.113 & 4.1615E-06 & 759.15 & 17.2597 & 3.248 & 5.1479E-06 \\
\hline 675.15 & 13.9254 & 3.173 & 2.1578E-06 & 759.15 & 17.9462 & 3.405 & 6.6893E-06 \\
\hline 675.15 & 14.1216 & 3.234 & 3.0826E-06 & 759.15 & 18.1423 & 3.451 & 6.8434E-06 \\
\hline 675.15 & 14.2196 & 3.264 & $2.8360 \mathrm{E}-06$ & 759.15 & 18.4365 & 3.520 & 6.4118E-06 \\
\hline 675.15 & 14.6119 & 3.388 & 2.9593E-06 & 759.15 & 18.6326 & 3.566 & 8.0148E-06 \\
\hline 675.15 & 14.6119 & 3.388 & 4.6239E-06 & 759.15 & 19.1230 & 3.682 & 1.0913E-05 \\
\hline 675.15 & 15.1022 & 3.547 & 4.3156E-06 & 759.15 & 19.2210 & 3.706 & 8.9088E-06 \\
\hline 675.15 & 15.1022 & 3.547 & 4.2540E-06 & 759.15 & 19.6133 & 3.801 & 7.5524E-06 \\
\hline 675.15 & 15.2003 & 3.580 & 5.7336E-06 & 759.15 & 19.6133 & 3.801 & 1.0173E-05 \\
\hline 675.15 & 15.3964 & 3.645 & 4.9630E-06 & 759.15 & 20.2017 & 3.944 & $1.2331 \mathrm{E}-05$ \\
\hline 675.15 & 15.5926 & 3.712 & 4.0074E-06 & 759.15 & 20.2998 & 3.969 & 1.3286E-05 \\
\hline 675.15 & 15.5926 & 3.712 & 7.3366E-06 & 759.15 & 20.4959 & 4.017 & 1.4797E-05 \\
\hline 675.15 & 15.7887 & 3.779 & $6.7817 E-06$ & 759.15 & 20.7901 & 4.091 & $1.1252 \mathrm{E}-05$ \\
\hline 675.15 & 15.7887 & 3.779 & 7.3983E-06 & 759.15 & 21.3785 & 4.240 & $1.5413 \mathrm{E}-05$ \\
\hline 675.15 & 15.8868 & 3.813 & 5.8570E-06 & 759.15 & 21.4766 & 4.265 & 1.9976E-05 \\
\hline 675.15 & 15.8868 & 3.813 & $6.0111 E-06$ & 759.15 & 21.9669 & 4.391 & $1.3872 E-05$ \\
\hline 675.15 & 16.1810 & 3.916 & 1.0573E-05 & 759.15 & 21.9669 & 4.391 & 2.2658E-05 \\
\hline 675.15 & 16.1810 & 3.916 & 8.6314E-06 & 759.15 & 22.4572 & 4.519 & 2.3722E-05 \\
\hline 675.15 & 16.2790 & 3.951 & 5.5487E-06 & 759.15 & 22.6534 & 4.571 & $2.0038 \mathrm{E}-05$ \\
\hline 675.15 & 16.3771 & 3.986 & 6.1652E-06 & 759.15 & 23.0456 & 4.676 & 1.8959E-05 \\
\hline 675.15 & 16.4752 & 4.022 & $9.1862 E-06$ & 759.15 & 23.1437 & 4.702 & $2.7806 \mathrm{E}-05$ \\
\hline 675.15 & 16.5732 & 4.057 & 8.9396E-06 & 759.15 & 23.6340 & 4.835 & 3.5144E-05 \\
\hline 675.15 & 16.6713 & 4.093 & 1.3718E-05 & 759.15 & 23.8302 & 4.888 & $2.5278 \mathrm{E}-05$ \\
\hline 675.15 & 16.7694 & 4.130 & 8.3231E-06 & 759.15 & 24.2224 & 4.997 & 2.2812E-05 \\
\hline 675.15 & 16.7694 & 4.130 & 1.4489E-05 & 759.15 & 24.5166 & 5.079 & 3.8566E-05 \\
\hline 675.15 & 16.8674 & 4.166 & $1.3564 \mathrm{E}-05$ & 759.15 & 24.9089 & 5.189 & 3.6069E-05 \\
\hline 675.15 & 17.1616 & 4.277 & 1.1406E-05 & 759.15 & 25.1050 & 5.245 & 3.6840E-05 \\
\hline 675.15 & 17.2597 & 4.315 & 8.0148E-06 & 759.15 & 25.3012 & 5.302 & 3.5761E-05 \\
\hline 759.15 & 8.0414 & 1.369 & 3.8532E-07 & 759.15 & 26.0857 & 5.530 & $5.6649 \mathrm{E}-05$ \\
\hline 759.15 & 8.0414 & 1.369 & 4.0073E-07 & 759.15 & 26.3799 & 5.617 & 4.8247E-05 \\
\hline 759.15 & 8.8260 & 1.514 & 7.0899E-07 & 820.15 & 6.4724 & 0.989 & 4.4697E-07 \\
\hline 759.15 & 9.3163 & 1.606 & $6.7817 \mathrm{E}-07$ & 820.15 & 6.8647 & 1.051 & 3.3600E-07 \\
\hline 759.15 & 9.3163 & 1.606 & $6.1651 E-07$ & 820.15 & 7.5511 & 1.162 & 2.7743E-07 \\
\hline 759.15 & 9.5124 & 1.643 & 3.3908E-07 & 820.15 & 8.1395 & 1.257 & 3.2367E-07 \\
\hline 759.15 & 10.1989 & 1.774 & 8.3229E-07 & 820.15 & 9.0221 & 1.402 & 4.0073E-07 \\
\hline 759.15 & 10.3950 & 1.811 & $1.1714 \mathrm{E}-06$ & 820.15 & 10.0028 & 1.565 & 8.6312E-07 \\
\hline 759.15 & 11.4738 & 2.022 & 1.3872E-06 & 820.15 & 10.4931 & 1.647 & 7.0899E-07 \\
\hline 759.15 & 12.6506 & 2.258 & $1.7571 \mathrm{E}-06$ & 820.15 & 10.5912 & 1.663 & 8.0147E-07 \\
\hline 759.15 & 12.9448 & 2.318 & $1.9420 \mathrm{E}-06$ & 820.15 & 11.4738 & 1.813 & $1.0172 \mathrm{E}-06$ \\
\hline 759.15 & 13.7293 & 2.479 & $2.1270 \mathrm{E}-06$ & 820.15 & 11.9641 & 1.897 & $1.7879 E-06$ \\
\hline 759.15 & 14.0235 & 2.541 & 2.9285E-06 & 820.15 & 12.0622 & 1.914 & $1.6646 \mathrm{E}-06$ \\
\hline 759.15 & 14.0235 & 2.541 & 2.9901E-06 & 820.15 & 12.4545 & 1.982 & 1.4796E-06 \\
\hline 759.15 & 14.3177 & 2.603 & 2.6818E-06 & 820.15 & 12.8467 & 2.050 & $1.5105 \mathrm{E}-06$ \\
\hline 759.15 & 14.9061 & 2.728 & $3.7608 \mathrm{E}-06$ & 820.15 & 13.4351 & 2.153 & 2.9593E-06 \\
\hline 759.15 & 15.1022 & 2.770 & 3.0826E-06 & 820.15 & 13.6312 & 2.188 & 1.8804E-06 \\
\hline 759.15 & 15.3964 & 2.834 & 4.0999E-06 & 820.15 & 14.2196 & 2.292 & 2.4661E-06 \\
\hline 759.15 & 15.7887 & 2.919 & 3.9457E-06 & 820.15 & 14.4158 & 2.327 & 2.4352E-06 \\
\hline
\end{tabular}




\begin{tabular}{crrr}
$\begin{array}{c}\text { Temperature } \\
\text { (K) }\end{array}$ & $\begin{array}{c}\text { Pressure } \\
\text { (MPa) }\end{array}$ & $\begin{array}{c}\text { Density } \\
\text { (mol/L) }\end{array}$ & $\begin{array}{c}\text { Solubility } \\
\text { (mole fraction) }\end{array}$ \\
\hline 820.15 & 14.5138 & 2.345 & $2.4969 \mathrm{E}-06$ \\
820.15 & 15.3964 & 2.504 & $2.2503 \mathrm{E}-06$ \\
820.15 & 15.5926 & 2.540 & $3.2367 \mathrm{E}-06$ \\
820.15 & 15.6906 & 2.557 & $2.8976 \mathrm{E}-06$ \\
820.15 & 15.8868 & 2.593 & $3.3600 \mathrm{E}-06$ \\
820.15 & 16.6713 & 2.737 & $4.3156 \mathrm{E}-06$ \\
820.15 & 16.7694 & 2.756 & $4.6856 \mathrm{E}-06$ \\
820.15 & 16.9655 & 2.792 & $4.1923 \mathrm{E}-06$ \\
820.15 & 17.1616 & 2.829 & $4.7472 \mathrm{E}-06$ \\
820.15 & 17.7500 & 2.939 & $5.6412 \mathrm{E}-06$ \\
820.15 & 18.3384 & 3.050 & $6.1652 \mathrm{E}-06$ \\
820.15 & 18.5346 & 3.088 & $5.0246 \mathrm{E}-06$ \\
820.15 & 18.8288 & 3.144 & $5.8261 \mathrm{E}-06$ \\
820.15 & 18.8288 & 3.144 & $6.2577 \mathrm{E}-06$ \\
820.15 & 19.6133 & 3.295 & $8.3847 \mathrm{E}-06$ \\
820.15 & 19.9075 & 3.352 & $7.3366 \mathrm{E}-06$ \\
820.15 & 20.0056 & 3.371 & $9.4945 \mathrm{E}-06$ \\
820.15 & 20.7901 & 3.526 & $1.0974 \mathrm{E}-05$ \\
820.15 & 21.0843 & 3.584 & $1.0789 \mathrm{E}-05$ \\
820.15 & 21.7708 & 3.721 & $9.9261 \mathrm{E}-06$ \\
820.15 & 22.3592 & 3.840 & $1.3101 \mathrm{E}-05$ \\
820.15 & 22.4572 & 3.860 & $1.5937 \mathrm{E}-05$ \\
820.15 & 23.4379 & 4.061 & $1.5968 \mathrm{E}-05$ \\
820.15 & 23.6340 & 4.102 & $1.5937 \mathrm{E}-05$ \\
820.15 & 25.1050 & 4.411 & $2.0500 \mathrm{E}-05$ \\
820.15 & 26.2818 & 4.665 & $2.3891 \mathrm{E}-05$ \\
867.15 & 9.1202 & 1.324 & $6.4734 \mathrm{E}-07$ \\
867.15 & 10.1008 & 1.474 & $7.0899 \mathrm{E}-07$ \\
867.15 & 12.5525 & 1.856 & $1.7571 \mathrm{E}-06$ \\
867.15 & 13.4351 & 1.996 & $1.9420 \mathrm{E}-06$ \\
867.15 & 13.7293 & 2.043 & $2.0345 \mathrm{E}-06$ \\
867.15 & 14.6119 & 2.185 & $2.2195 \mathrm{E}-06$ \\
867.15 & 15.7887 & 2.377 & $2.4352 \mathrm{E}-06$ \\
867.15 & 15.8868 & 2.393 & $3.1442 \mathrm{E}-06$ \\
867.15 & 16.8674 & 2.555 & $3.9149 \mathrm{E}-06$ \\
867.15 & 17.0636 & 2.587 & $3.7608 \mathrm{E}-06$ \\
867.15 & 18.1423 & 2.768 & $4.8089 \mathrm{E}-06$ \\
867.15 & 18.2404 & 2.784 & $5.1479 \mathrm{E}-06$ \\
867.15 & 19.4172 & 2.984 & $6.4735 \mathrm{E}-06$ \\
867.15 & 19.4172 & 2.984 & $6.5351 \mathrm{E}-06$ \\
867.15 & 20.4959 & 3.170 & $8.0765 \mathrm{E}-06$ \\
867.15 & 20.4959 & 3.170 & $7.6141 \mathrm{E}-06$ \\
867.15 & 21.7708 & 3.392 & $8.8163 \mathrm{E}-06$ \\
867.15 & 21.8688 & 3.409 & $1.0851 \mathrm{E}-05$ \\
\hline & 23.1437 & 3.636 & $1.1930 \mathrm{E}-05$ \\
867.15 & 25.0070 & 3.972 & $1.8804 \mathrm{E}-05$ \\
\hline & & & \\
8 & &
\end{tabular}

Reference: Martynova, O.I. and Samoilov, Yu.F., 1962, The

12 formation of solutions of inorganic substances in water vapour: Russian Journal of Inorganic Chemistry, v. 7, n. 4, p. 372-375

Units of original measurements: Temperature in degrees $C$, Pressure in atm, Solubility in $\mathrm{mg} / \mathrm{kg}$

Temperature Pressure Density Solubility

$\begin{array}{llll}(\mathrm{K}) & (\mathrm{MPa}) \quad(\mathrm{mol} / \mathrm{L}) \quad(\text { mole fraction) }\end{array}$

\begin{tabular}{lrrr}
610.15 & 10.1325 & 2.671 & $7.4598 \mathrm{E}-07$ \\
722.15 & 14.1855 & 2.813 & $3.3292 \mathrm{E}-06$ \\
725.15 & 14.1855 & 2.791 & $3.6683 \mathrm{E}-06$ \\
771.15 & 14.1855 & 2.510 & $3.2059 \mathrm{E}-06$ \\
771.15 & 14.1855 & 2.510 & $3.5142 \mathrm{E}-06$ \\
772.15 & 7.3967 & 1.224 & $6.7200 \mathrm{E}-07$ \\
773.15 & 14.1855 & 2.500 & $3.0826 \mathrm{E}-06$ \\
773.15 & 18.2385 & 3.355 & $6.7509 \mathrm{E}-06$ \\
773.15 & 18.2385 & 3.355 & $7.0900 \mathrm{E}-06$ \\
773.15 & 18.2385 & 3.355 & $6.7509 \mathrm{E}-06$ \\
773.15 & 18.2385 & 3.355 & $6.9975 \mathrm{E}-06$ \\
773.15 & 18.2385 & 3.355 & $6.7509 \mathrm{E}-06$ \\
773.15 & 18.2385 & 3.355 & $6.7817 \mathrm{E}-06$ \\
774.15 & 14.1855 & 2.494 & $3.0826 \mathrm{E}-06$ \\
775.15 & 7.3967 & 1.218 & $7.0283 \mathrm{E}-07$ \\
775.15 & 14.1855 & 2.489 & $3.1442 \mathrm{E}-06$ \\
778.15 & 14.1855 & 2.474 & $3.2367 \mathrm{E}-06$ \\
784.15 & 10.1325 & 1.683 & $1.2639 \mathrm{E}-06$ \\
812.15 & 18.2385 & 3.080 & $7.8607 \mathrm{E}-06$ \\
813.15 & 18.2385 & 3.074 & $8.0148 \mathrm{E}-06$ \\
823.15 & 10.1325 & 1.579 & $2.3119 \mathrm{E}-06$ \\
823.15 & 18.2385 & 3.014 & $8.1690 \mathrm{E}-06$ \\
825.15 & 14.1855 & 2.267 & $3.1689 \mathrm{E}-06$ \\
830.15 & 14.1855 & 2.247 & $4.7780 \mathrm{E}-06$ \\
\hline & & &
\end{tabular}

Reference: Bell, M.J., Mravich, N.J., Pocock, F.J. and Rubright, M.M., 1977, Solids behavior in once13 through nuclear steam systems: Proceedings of the American Power Conference, v. 39, p. 849867

Units of original measurements: Temperature in degrees $F$, Pressure in psia, Solubility in $\mathrm{ppb}$ of $\mathrm{Na}$

Temperature Pressure Density Solubility

\begin{tabular}{ccrc}
$(\mathrm{K})$ & $(\mathrm{MPa})$ & $(\mathrm{mol} / \mathrm{L})$ & (mole fraction) \\
\hline 573.71 & 6.3776 & 1.656 & $2.9778 \mathrm{E}-08$ \\
598.71 & 7.3291 & 1.802 & $2.8210 \mathrm{E}-07$ \\
599.82 & 7.3429 & 1.799 & $2.7505 \mathrm{E}-07$ \\
600.93 & 8.1082 & 2.039 & $5.3286 \mathrm{E}-07$ \\
\hline
\end{tabular}

Reference: Allmon, W.E., et. al., 1983, Deposition of

14 Research Institute Research Project 1068-1 (NP3002)

Units of original measurements: Temperature in degrees $F$,

Pressure in psia, Solubility in wt\%

Temperature Pressure Density Solubility $(\mathrm{K}) \quad(\mathrm{MPa}) \quad(\mathrm{mol} / \mathrm{L}) \quad$ (mole fraction) $\begin{array}{llll}499.26 & 2.3787 & 0.650 & 2.1886 \mathrm{E}-09\end{array}$ 


\begin{tabular}{cccc}
$\begin{array}{c}\text { Temperature } \\
(\mathrm{K})\end{array}$ & $\begin{array}{c}\text { Pressure } \\
(\mathrm{MPa})\end{array}$ & $\begin{array}{c}\text { Density } \\
\text { (mol/L) }\end{array}$ & $\begin{array}{c}\text { Solubility } \\
\text { (mole fraction) }\end{array}$ \\
\hline 510.37 & 2.9992 & 0.822 & $3.6991 \mathrm{E}-09$ \\
515.93 & 3.2750 & 0.895 & $6.7816 \mathrm{E}-09$ \\
517.59 & 3.5163 & 0.971 & $4.3156 \mathrm{E}-09$ \\
519.82 & 3.6542 & 1.010 & $6.7816 \mathrm{E}-09$ \\
523.15 & 3.2750 & 0.872 & $2.6510 \mathrm{E}-09$ \\
524.26 & 3.7576 & 1.027 & $1.2639 \mathrm{E}-08$ \\
525.37 & 3.3440 & 0.887 & $5.8569 \mathrm{E}-09$ \\
534.26 & 3.9645 & 1.056 & $6.4734 \mathrm{E}-09$ \\
535.37 & 4.5505 & 1.251 & $1.8804 \mathrm{E}-08$ \\
544.26 & 4.5850 & 1.218 & $1.1097 \mathrm{E}-08$ \\
\hline
\end{tabular}

\begin{tabular}{cccc}
$\begin{array}{c}\text { Temperature } \\
(\mathrm{K})\end{array}$ & $\begin{array}{c}\text { Pressure } \\
(\mathrm{MPa})\end{array}$ & $\begin{array}{c}\text { Density } \\
(\mathrm{mol} / \mathrm{L})\end{array}$ & $\begin{array}{c}\text { Solubility } \\
\text { (mole fraction) }\end{array}$ \\
\hline 721.15 & 24.2000 & 5.807 & $7.7077 \mathrm{E}-05$ \\
723.15 & 18.7000 & 3.984 & $7.3983 \mathrm{E}-06$ \\
723.15 & 22.4000 & 5.123 & $4.3777 \mathrm{E}-05$ \\
723.15 & 25.4000 & 6.203 & $8.3245 \mathrm{E}-05$ \\
\hline
\end{tabular}
Reference: Urusova, M.A., 1974, Phase equilibria in the sodium hydroxide-water and sodium chloride- 63 water systems at 350-550 C: Russian Journal of Inorganic Chemistry, v. 19 , n. 3, p. 450-454
Units of original measurements: Temperature in degrees $C$, Pressure in $\mathrm{kgt} / \mathrm{cm} 2$, Solubility in mole \%

\begin{tabular}{cccc}
$\begin{array}{c}\text { Temperature } \\
(\mathrm{K})\end{array}$ & $\begin{array}{c}\text { Pressure } \\
(\mathrm{MPa})\end{array}$ & $\begin{array}{c}\text { Density } \\
(\mathrm{mol} / \mathrm{L})\end{array}$ & $\begin{array}{c}\text { Solubility } \\
\text { (mole fraction) }\end{array}$ \\
\hline 723.15 & 41.3841 & 16.179 & $9.4000 \mathrm{E}-03$ \\
723.15 & 42.0705 & 16.742 & $1.6000 \mathrm{E}-02$ \\
723.15 & 42.1686 & 16.822 & $2.4000 \mathrm{E}-02$ \\
723.15 & 42.2667 & 16.901 & $2.4000 \mathrm{E}-02$ \\
773.15 & 57.5650 & 17.751 & $2.6000 \mathrm{E}-02$ \\
773.15 & 58.0554 & 17.966 & $3.3000 \mathrm{E}-02$ \\
773.15 & 58.3496 & 18.095 & $4.0000 \mathrm{E}-02$ \\
823.15 & 74.0402 & 18.434 & $4.2000 \mathrm{E}-02$ \\
823.15 & 74.5305 & 18.577 & $5.2000 \mathrm{E}-02$ \\
823.15 & 74.6286 & 18.605 & $5.2000 \mathrm{E}-02$ \\
\hline
\end{tabular}

Reference: Bischoff, J.L., Rosenbauer, R.J. and Pitzer, 15 K.S., 1986, The system $\mathrm{NaCl}-\mathrm{H} 2 \mathrm{O}$ : relations of vapor-liquid near the critical temperature of water and of vapor-liquid-halite from 300 to $500 \mathrm{C}$ : Geochimica et Cosmochimica Acta, v. 50, p. 1437-1444

Units of original measurements: Temperature in degrees $C$, Pressure in bar, Solubility in wt\%

\begin{tabular}{crrr}
$\begin{array}{c}\text { Temperature } \\
(\mathrm{K})\end{array}$ & $\begin{array}{c}\text { Pressure } \\
(\mathrm{MPa})\end{array}$ & $\begin{array}{c}\text { Density } \\
(\mathrm{mol} / \mathrm{L})\end{array}$ & $\begin{array}{c}\text { Solubility } \\
\text { (mole fraction) }\end{array}$ \\
\hline 596.85 & 7.8800 & 1.995 & $2.1886 \mathrm{E}-06$ \\
621.65 & 10.5300 & 2.675 & $4.3156 \mathrm{E}-06$ \\
648.25 & 13.7400 & 3.483 & $9.8644 \mathrm{E}-06$ \\
648.65 & 13.8400 & 3.514 & $8.0148 \mathrm{E}-06$ \\
673.35 & 17.2700 & 4.358 & $2.0038 \mathrm{E}-05$ \\
700.15 & 21.4100 & 5.323 & $4.0077 \mathrm{E}-05$ \\
723.65 & 25.1500 & 6.091 & $8.3245 \mathrm{E}-05$ \\
724.95 & 25.3500 & 6.127 & $9.5580 \mathrm{E}-05$ \\
749.05 & 28.8800 & 6.679 & $1.4184 \mathrm{E}-04$ \\
750.15 & 29.1900 & 6.754 & $1.5110 \mathrm{E}-04$ \\
775.65 & 32.7200 & 7.164 & $2.1588 \mathrm{E}-04$ \\
776.55 & 32.8000 & 7.163 & $2.3131 \mathrm{E}-04$ \\
\hline
\end{tabular}

Reference: Wofford, W.T., Dell'Orco, P.C. and Gloyna, E.F., 1995, Solubility of potassium hydroxide and 16 potassium phosphate in supercritical water: Journal of Chemical and Engineering Data, v. 40, p. $968-973$

Units of original measurements: Temperature in degrees $C$, Pressure in $\mathrm{MPa}$, Solubility in $\mathrm{mg} / \mathrm{kg}$

\begin{tabular}{cccc}
$\begin{array}{c}\text { Temperature } \\
(\mathrm{K})\end{array}$ & $\begin{array}{c}\text { Pressure } \\
(\mathrm{MPa})\end{array}$ & $\begin{array}{c}\text { Density } \\
(\mathrm{mol} / \mathrm{L})\end{array}$ & $\begin{array}{c}\text { Solubility } \\
\text { (mole fraction) }\end{array}$ \\
\hline 723.15 & 24.8000 & 5.973 & $7.1835 \mathrm{E}-05$ \\
743.15 & 25.0000 & 5.534 & $6.0118 \mathrm{E}-05$ \\
\hline
\end{tabular}

Reference: Baierlein, H., 1983, Zur Loslichkeit von Salzen in 18 Dissertation - Erlangen

Units of original measurements: Temperature in degrees $C$, Pressure in bar, Solubility in Wt\%, Data set excluded from equation fit

\begin{tabular}{ccrc}
$\begin{array}{c}\text { Temperature } \\
(\mathrm{K})\end{array}$ & $\begin{array}{c}\text { Pressure } \\
(\mathrm{MPa})\end{array}$ & $\begin{array}{c}\text { Density } \\
(\mathrm{mol} / \mathrm{L})\end{array}$ & $\begin{array}{c}\text { Solubility } \\
(\text { mole fraction })\end{array}$ \\
\hline 721.15 & 17.5000 & 3.674 & $1.5105 \mathrm{E}-05$
\end{tabular}


Compound: Sodium Hydroxide $(\mathrm{NaOH})$

Reference: Sastry, V., 1957, Die Loslichkeit von Salzen in Hochgespanntem Wasserdampf und die 11 Klarung der Frage der Turbinenversalzung: Doctoral Dissertation - Technische Hochschule Karlsruhe

Units of original measurements: Temperature in degrees $C$, Pressure in $\mathrm{kgf} / \mathrm{cm} 2$, Solubility in $\mathrm{mg} / \mathrm{kg}$

\begin{tabular}{cccc}
$\begin{array}{c}\text { Temperature } \\
(\mathrm{K})\end{array}$ & $\begin{array}{c}\text { Pressure } \\
\text { (MPa) }\end{array}$ & $\begin{array}{c}\text { Density } \\
\text { (mol/L) }\end{array}$ & $\begin{array}{c}\text { Solubility } \\
\text { (mole fraction) }\end{array}$ \\
\hline 675.15 & 5.9821 & 1.162 & $3.2430 \mathrm{E}-07$ \\
675.15 & 7.0608 & 1.397 & $4.5492 \mathrm{E}-07$ \\
675.15 & 7.9434 & 1.596 & $4.9546 \mathrm{E}-07$ \\
675.15 & 8.5318 & 1.732 & $6.8013 \mathrm{E}-07$ \\
675.15 & 8.8260 & 1.802 & $2.0719 \mathrm{E}-07$ \\
675.15 & 9.6105 & 1.992 & $6.9364 \mathrm{E}-07$ \\
675.15 & 10.2970 & 2.163 & $1.4864 \mathrm{E}-06$ \\
675.15 & 10.3950 & 2.188 & $1.0810 \mathrm{E}-06$ \\
675.15 & 11.1796 & 2.392 & $9.9091 \mathrm{E}-07$ \\
675.15 & 12.1603 & 2.658 & $1.3963 \mathrm{E}-06$ \\
675.15 & 12.2583 & 2.685 & $1.6215 \mathrm{E}-06$ \\
675.15 & 12.8467 & 2.852 & $2.6124 \mathrm{E}-06$ \\
675.15 & 13.0428 & 2.909 & $2.1170 \mathrm{E}-06$ \\
675.15 & 13.8274 & 3.143 & $2.7926 \mathrm{E}-06$ \\
675.15 & 14.0235 & 3.203 & $3.7835 \mathrm{E}-06$ \\
675.15 & 14.7100 & 3.419 & $4.4591 \mathrm{E}-06$ \\
675.15 & 15.4945 & 3.678 & $6.3509 \mathrm{E}-06$ \\
675.15 & 15.6906 & 3.745 & $5.4050 \mathrm{E}-06$ \\
675.15 & 16.1810 & 3.916 & $4.5492 \mathrm{E}-06$ \\
675.15 & 16.2790 & 3.951 & $8.6931 \mathrm{E}-06$ \\
675.15 & 16.6713 & 4.093 & $6.7563 \mathrm{E}-06$ \\
\hline
\end{tabular}

Reference: Bell, M.J., Mravich, N.J., Pocock, F.J. and 13 Rubright, M.M., 1977, Solids behavior in oncethrough nuclear steam systems: Proceedings of the American Power Conference, v. 39, p. 849 867

Units of original measurements: Temperature in degrees $F$, Pressure in psia, Solubility in ppb of $\mathrm{Na}$

\begin{tabular}{cccc}
$\begin{array}{c}\text { Temperature } \\
(\mathrm{K})\end{array}$ & $\begin{array}{c}\text { Pressure } \\
(\mathrm{MPa})\end{array}$ & $\begin{array}{c}\text { Density } \\
(\mathrm{mol} / \mathrm{L})\end{array}$ & $\begin{array}{c}\text { Solubility } \\
\text { (mole fraction) }\end{array}$ \\
\hline 535.37 & 1.1859 & 0.277 & $7.8362 \mathrm{E}-09$ \\
535.37 & 1.3376 & 0.314 & $1.8807 \mathrm{E}-08$ \\
535.37 & 1.5720 & 0.373 & $1.4105 \mathrm{E}-08$ \\
547.04 & 0.9239 & 0.209 & $4.7017 \mathrm{E}-09$ \\
575.93 & 6.5155 & 1.688 & $1.5281 \mathrm{E}-07$ \\
589.82 & 7.2533 & 1.839 & $4.2316 \mathrm{E}-07$ \\
600.37 & 7.3222 & 1.789 & $5.8772 \mathrm{E}-07$ \\
602.59 & 7.3567 & 1.786 & $5.8772 \mathrm{E}-07$ \\
\hline
\end{tabular}

Reference: Allmon, W.E., et. al., 1983, Deposition of corrosive salts from steam: Electric Power 14 Research Institute Research Project 1068-1 (NP. 3002)

Units of original measurements: Temperature in degrees $F$, Pressure in psia, Solubility in wt\%

Temperature Pressure Density Solubility (K) $\quad(\mathrm{MPa}) \quad(\mathrm{mol} / \mathrm{L}) \quad$ (mole fraction)

\begin{tabular}{llll}
403.15 & 0.1586 & 0.048 & $4.5041 \mathrm{E}-10$ \\
404.82 & 0.1586 & 0.048 & $1.8017 \mathrm{E}-10$ \\
464.82 & 0.3654 & 0.097 & $2.4322 \mathrm{E}-10$ \\
470.37 & 0.9997 & 0.271 & $7.2066 \mathrm{E}-10$ \\
470.93 & 1.4479 & 0.406 & $3.0628 \mathrm{E}-09$ \\
471.48 & 1.0204 & 0.277 & $7.2066 \mathrm{E}-10$ \\
472.59 & 1.0135 & 0.274 & $9.4587 \mathrm{E}-10$ \\
473.15 & 0.9928 & 0.267 & $6.3058 \mathrm{E}-10$ \\
474.26 & 1.0204 & 0.274 & $1.5765 \mathrm{E}-09$ \\
495.37 & 1.9374 & 0.520 & $1.0179 \mathrm{E}-08$ \\
497.04 & 2.4132 & 0.666 & $7.9273 \mathrm{E}-09$ \\
497.04 & 2.4476 & 0.677 & $5.4050 \mathrm{E}-09$ \\
504.26 & 2.6200 & 0.715 & $7.6570 \mathrm{E}-09$ \\
507.04 & 2.3787 & 0.633 & $2.8827 \mathrm{E}-09$ \\
510.37 & 0.9859 & 0.242 & $7.6570 \mathrm{E}-10$ \\
511.48 & 2.7096 & 0.725 & $4.0087 \mathrm{E}-09$ \\
511.48 & 2.7510 & 0.738 & $3.6934 \mathrm{E}-09$ \\
512.59 & 2.0753 & 0.534 & $2.2521 \mathrm{E}-09$ \\
512.59 & 2.0822 & 0.536 & $2.2521 \mathrm{E}-09$ \\
514.82 & 0.9997 & 0.243 & $6.3058 \mathrm{E}-10$ \\
517.04 & 0.9515 & 0.230 & $7.6570 \mathrm{E}-10$ \\
517.04 & 1.0066 & 0.243 & $7.6570 \mathrm{E}-10$ \\
517.04 & 1.7306 & 0.432 & $8.5579 \mathrm{E}-10$ \\
519.26 & 1.7857 & 0.445 & $1.9368 \mathrm{E}-09$ \\
519.82 & 1.7857 & 0.444 & $2.1169 \mathrm{E}-09$ \\
520.37 & 1.7788 & 0.441 & $2.2070 \mathrm{E}-09$ \\
522.04 & 3.6197 & 0.989 & $1.2927 \mathrm{E}-08$ \\
527.04 & 3.4680 & 0.921 & $1.1756 \mathrm{E}-08$ \\
555.93 & 5.5158 & 1.474 & $1.2522 \mathrm{E}-08$ \\
572.04 & 4.5160 & 1.089 & $6.2608 \mathrm{E}-08$ \\
573.71 & 2.8268 & 0.640 & $3.0628 \mathrm{E}-09$ \\
573.71 & 4.9504 & 1.207 & $7.9723 \mathrm{E}-08$ \\
574.82 & 1.7030 & 0.372 & $1.5765 \mathrm{E}-09$ \\
\hline & & & \\
\hline
\end{tabular}

Reference: Urusova, M.A., 1974, Phase equilibria in the sodium hydroxide-water and sodium chloride63 water systems at 350-550 C: Russian Journal of Inorganic Chemistry, v. 19, n. 3, p. 450-454

Units of original measurements: Temperature in degrees $C$, Pressure in $\mathrm{kgf} / \mathrm{cm} 2$, Solubility in mole \%

Temperature Pressure Density Solubility

\begin{tabular}{clrr}
$(\mathrm{K})$ & $(\mathrm{MPa})$ & $(\mathrm{mol} / \mathrm{L})$ & (mole fraction) \\
\hline 723.15 & 44.5222 & 18.680 & $2.3000 \mathrm{E}-02$ \\
723.15 & 44.5222 & 18.680 & $2.4000 \mathrm{E}-02$ \\
723.15 & 44.7183 & 18.829 & $2.3000 \mathrm{E}-02$ \\
723.15 & 44.8164 & 18.902 & $2.3000 \mathrm{E}-02$ \\
723.15 & 45.8951 & 19.692 & $3.2000 \mathrm{E}-02$
\end{tabular}




\begin{tabular}{cccc}
$\begin{array}{c}\text { Temperature } \\
(\mathrm{K})\end{array}$ & $\begin{array}{c}\text { Pressure } \\
(\mathrm{MPa})\end{array}$ & $\begin{array}{c}\text { Density } \\
(\mathrm{mol} / \mathrm{L})\end{array}$ & $\begin{array}{c}\text { Solubility } \\
\text { (mole fraction) }\end{array}$ \\
\hline 773.15 & 63.9394 & 20.390 & $3.8000 \mathrm{E}-02$ \\
773.15 & 64.3316 & 20.540 & $3.8000 \mathrm{E}-02$ \\
773.15 & 65.9007 & 21.124 & $4.7000 \mathrm{E}-02$ \\
773.15 & 67.1756 & 21.580 & $6.0000 \mathrm{E}-02$ \\
\hline
\end{tabular}

Compound: Sodium Phosphate (Na3PO4)

Reference: Wetton, E.A.M., 1981, Phase equlilibria in

27 aqueous sodium phosphate solutions at $350 \mathrm{C}$

27 Power Industry Research, v. 1, p. 151-158

Units of original measurements: Temperature in degrees $C$,

Solubility in wt\%, Original data in graphical format, all data points

are at saturation

\begin{tabular}{cccc}
$\begin{array}{c}\text { Temperature } \\
(\mathrm{K})\end{array}$ & $\begin{array}{c}\text { Pressure } \\
(\mathrm{MPa})\end{array}$ & $\begin{array}{c}\text { Density } \\
(\mathrm{mol} / \mathrm{L})\end{array}$ & $\begin{array}{c}\text { Solubility } \\
\text { (mole fraction })\end{array}$ \\
\hline 623.15 & 16.5294 & 31.901 & $5.9690 \mathrm{E}-04$ \\
\hline
\end{tabular}

Reference: Schroeder, W.C., Berk, A.A. and Gabriel, A.,

$28 \quad 1937$, Solubility equilibria of sodium sulfate at temperatures from 150 to 350 degrees. III. Effect of sodium hydroxide and sodium phosphate: Journal of the American Chemical Society, v. 59, p. $1783-1790$

Units of original measurements: Temperature in degrees $C$, Solubility in wt\%, all data points are at saturation

\begin{tabular}{cccc}
$\begin{array}{c}\text { Temperature } \\
(\mathrm{K})\end{array}$ & $\begin{array}{c}\text { Pressure } \\
(\mathrm{MPa})\end{array}$ & $\begin{array}{c}\text { Density } \\
(\mathrm{mol} / \mathrm{L})\end{array}$ & $\begin{array}{c}\text { Solubility } \\
\text { (mole fraction) }\end{array}$ \\
\hline 487.15 & 2.0650 & 47.059 & $5.2083 \mathrm{E}-02$ \\
487.15 & 2.0650 & 47.059 & $5.2872 \mathrm{E}-02$ \\
489.15 & 2.1473 & 46.921 & $5.0896 \mathrm{E}-02$ \\
489.15 & 2.1473 & 46.921 & $4.9707 \mathrm{E}-02$ \\
498.15 & 2.5497 & 46.280 & $2.6946 \mathrm{E}-02$ \\
498.15 & 2.5497 & 46.280 & $2.9644 \mathrm{E}-02$ \\
498.15 & 2.5497 & 46.280 & $2.9126 \mathrm{E}-02$ \\
498.15 & 2.5497 & 46.280 & $3.5710 \mathrm{E}-02$ \\
508.15 & 3.0625 & 45.535 & $1.9291 \mathrm{E}-02$ \\
523.15 & 3.9762 & 44.345 & $9.2541 \mathrm{E}-03$ \\
523.15 & 3.9762 & 44.345 & $9.3620 \mathrm{E}-03$ \\
523.15 & 3.9762 & 44.345 & $9.3620 \mathrm{E}-03$ \\
573.15 & 8.5879 & 39.530 & $2.6304 \mathrm{E}-03$ \\
623.15 & 16.5294 & 31.901 & $1.6481 \mathrm{E}-04$ \\
\hline
\end{tabular}


Compound: Sodium Sulfate (Na2SO4)

\begin{tabular}{|c|c|c|c|}
\hline \multicolumn{4}{|c|}{$\begin{array}{cl}\text { Reference: } & \begin{array}{l}\text { Armellini, F.J. and Tester. J.W., 1993, Solubility } \\
\text { of sodium chloride and sulfate in sub-and }\end{array} \\
0.5 & \text { supercritical water vapor from } 450-550 \mathrm{C} \text { and } \\
& 100-250 \text { bar: Fluid Phase Equilibria, v. 84. p. } \\
& 123-142\end{array}$} \\
\hline \multicolumn{4}{|c|}{$\begin{array}{l}\text { Units of original measurements: Temperature in degrees } C \text {, } \\
\text { Pressure in bar, Solubility in ppm }\end{array}$} \\
\hline $\begin{array}{l}\text { Temperatur } \\
\text { (K) }\end{array}$ & $\begin{array}{c}\text { Pressure } \\
(\mathrm{MPa})\end{array}$ & $\begin{array}{l}\text { Density } \\
(\mathrm{mol} / \mathrm{L})\end{array}$ & $\begin{array}{l}\text { Solubility } \\
\text { (mole fraction) }\end{array}$ \\
\hline & 25.0000 & 4.982 & 1.2049E-07 \\
\hline
\end{tabular}

Reference: Styrikovich, M.A., Khaibullin, I.Kh. and

08 high pressure steam: Dokl. Akad. Nauk SSSR, v. 100, p. $1123-1126$

Units of original measurements: Temperature in degrees $C$, Pressure in atm, Solubility in $\mathrm{mg} / \mathrm{kg}$, Original data in graphical format

Temperature Pressure Density Solubility

\begin{tabular}{rrrr}
\multicolumn{1}{c}{$(\mathrm{K})$} & \multicolumn{1}{c}{$(\mathrm{MPa})$} & $(\mathrm{mol} / \mathrm{L})$ & (mole fraction) \\
\hline 601.08 & 10.1325 & 2.803 & $1.9358 \mathrm{E}-09$ \\
623.36 & 14.1855 & 4.296 & $2.5766 \mathrm{E}-09$ \\
627.02 & 10.1325 & 2.479 & $1.6688 \mathrm{E}-09$ \\
640.10 & 3.0398 & 0.601 & $2.5766 \mathrm{E}-09$ \\
642.92 & 14.1855 & 3.760 & $1.6688 \mathrm{E}-09$ \\
643.02 & 18.2385 & 6.070 & $1.0053 \mathrm{E}-08$ \\
643.23 & 10.1325 & 2.336 & $2.1761 \mathrm{E}-09$ \\
654.74 & 18.2385 & 5.395 & $5.5939 \mathrm{E}-09$ \\
655.58 & 20.2650 & 6.735 & $1.8263 \mathrm{E}-08$ \\
661.02 & 3.0398 & 0.579 & $1.8958 \mathrm{E}-09$ \\
661.64 & 10.1325 & 2.204 & $2.4298 \mathrm{E}-09$ \\
662.59 & 14.1855 & 3.420 & $2.9505 \mathrm{E}-09$ \\
681.73 & 14.1855 & 3.177 & $4.3256 \mathrm{E}-09$ \\
683.30 & 20.2650 & 5.344 & $8.0103 \mathrm{E}-09$ \\
683.40 & 10.1325 & 2.076 & $4.8195 \mathrm{E}-09$ \\
684.03 & 3.0398 & 0.556 & $3.8716 \mathrm{E}-09$ \\
703.38 & 18.2385 & 4.135 & $5.5271 \mathrm{E}-09$ \\
705.05 & 14.1855 & 2.949 & $6.0878 \mathrm{E}-09$ \\
705.05 & 20.2650 & 4.780 & $6.5150 \mathrm{E}-09$ \\
705.79 & 10.1325 & 1.966 & $5.1666 \mathrm{E}-09$ \\
706.73 & 3.0398 & 0.535 & $5.5405 \mathrm{E}-09$ \\
709.97 & 18.2385 & 4.033 & $5.5271 \mathrm{E}-09$ \\
729.53 & 3.0398 & 0.516 & $7.3027 \mathrm{E}-09$ \\
732.36 & 10.1325 & 1.856 & $8.2106 \mathrm{E}-09$ \\
732.77 & 20.2650 & 4.295 & $8.8514 \mathrm{E}-09$ \\
733.61 & 14.1855 & 2.732 & $7.4896 \mathrm{E}-09$ \\
733.72 & 18.2385 & 3.728 & $7.8367 \mathrm{E}-09$ \\
765.93 & 18.2385 & 3.414 & $8.7846 \mathrm{E}-09$ \\
766.14 & 3.0398 & 0.489 & $9.8927 \mathrm{E}-09$ \\
766.14 & 10.1325 & 1.738 & $1.1882 \mathrm{E}-08$ \\
786.96 & 3.0398 & 0.475 & $1.4245 \mathrm{E}-08$ \\
790.51 & 14.1855 & 2.414 & $1.3204 \mathrm{E}-08$ \\
790.51 & 18.2385 & 3.223 & $1.3711 \mathrm{E}-08$ \\
791.14 & 10.1325 & 1.663 & $1.4192 \mathrm{E}-08$
\end{tabular}

Temperature Pressure Density Solubility

\begin{tabular}{crrr}
$(\mathrm{K})$ & \multicolumn{1}{c}{$(\mathrm{MPa})$} & $(\mathrm{mol} / \mathrm{L})$ & $($ mole fraction $)$ \\
\hline 815.30 & 18.2385 & 3.060 & $1.5700 \mathrm{E}-08$ \\
815.72 & 3.0398 & 0.457 & $1.7262 \mathrm{E}-08$ \\
816.35 & 14.1855 & 2.302 & $1.6568 \mathrm{E}-08$ \\
816.98 & 10.1325 & 1.594 & $1.6661 \mathrm{E}-08$ \\
\hline
\end{tabular}

Reference: Styrikovich, M.A., 1957, Solubility of salts in steam of supercritical characteristics:

10 Teploenergetika, v. 4, n. 2, p. 3-7

Units of original measurements: Temperature in degrees $C$, Pressure in $\mathrm{kgf} / \mathrm{cm} 2$, Solubility in $\mathrm{mg} / \mathrm{kg}$, Original data in graphical format

Temperature Pressure Density Solubility (K) $\quad(\mathrm{MPa}) \quad(\mathrm{mol} / \mathrm{L}) \quad$ (mole fraction)

$\begin{array}{lrrr}668.15 & 27.4586 & 16.433 & 5.0098 \mathrm{E}-07 \\ 675.53 & 25.4973 & 9.416 & 2.9223 \mathrm{E}-08 \\ 675.53 & 25.4973 & 9.416 & 1.8235 \mathrm{E}-08 \\ 678.57 & 25.4973 & 8.979 & 2.5132 \mathrm{E}-08 \\ 680.43 & 23.5360 & 7.246 & 1.1689 \mathrm{E}-08 \\ 681.60 & 23.5360 & 7.166 & 1.1339 \mathrm{E}-08 \\ 681.60 & 27.4586 & 10.602 & 3.8575 \mathrm{E}-08 \\ 681.83 & 23.5360 & 7.151 & 8.7670 \mathrm{E}-09 \\ 683.00 & 27.4586 & 10.339 & 6.5460 \mathrm{E}-08 \\ 684.17 & 29.4200 & 12.646 & 9.0242 \mathrm{E}-08 \\ 684.63 & 21.5746 & 5.909 & 7.7150 \mathrm{E}-09 \\ 685.10 & 29.4200 & 12.378 & 9.2931 \mathrm{E}-08 \\ 685.57 & 29.4200 & 12.251 & 4.7108 \mathrm{E}-08 \\ 686.27 & 21.5746 & 5.844 & 7.8319 \mathrm{E}-09 \\ 690.24 & 21.5746 & 5.699 & 8.8839 \mathrm{E}-09 \\ 699.57 & 29.4200 & 9.806 & 1.6365 \mathrm{E}-08 \\ 704.24 & 29.4200 & 9.310 & 1.1689 \mathrm{E}-08 \\ 707.51 & 27.4586 & 7.838 & 6.8967 \mathrm{E}-09 \\ 707.74 & 29.4200 & 8.993 & 6.0785 \mathrm{E}-09 \\ 709.85 & 25.4973 & 6.732 & 5.1433 \mathrm{E}-09 \\ 718.01 & 23.5360 & 5.647 & 1.9872 \mathrm{E}-09 \\ 718.25 & 23.5360 & 5.640 & 6.6629 \mathrm{E}-09 \\ 719.65 & 23.5360 & 5.603 & 2.2210 \mathrm{E}-09 \\ 722.68 & 23.5360 & 5.525 & 7.2474 \mathrm{E}-09 \\ 724.32 & 23.5360 & 5.484 & 2.6886 \mathrm{E}-09 \\ 727.35 & 21.5746 & 4.773 & 2.2210 \mathrm{E}-09 \\ 728.52 & 21.5746 & 4.752 & 8.2995 \mathrm{E}-09 \\ 731.32 & 21.5746 & 4.703 & 3.0392 \mathrm{E}-09 \\ 731.79 & 21.5746 & 4.695 & 4.2082 \mathrm{E}-09 \\ 732.49 & 21.5746 & 4.682 & 5.1433 \mathrm{E}-09 \\ 753.03 & 25.4973 & 5.478 & 2.9223 \mathrm{E}-09 \\ 760.03 & 23.5360 & 4.794 & 2.4548 \mathrm{E}-09 \\ 760.26 & 21.5746 & 4.276 & 5.4940 \mathrm{E}-09 \\ 760.50 & 21.5746 & 4.273 & 4.0913 \mathrm{E}-09 \\ 768.20 & 21.5746 & 4.179 & 4.4420 \mathrm{E}-09 \\ 769.84 & 25.4973 & 5.167 & 8.2995 \mathrm{E}-09 \\ 770.30 & 23.5360 & 4.644 & 3.1561 \mathrm{E}-09 \\ 776.61 & 27.4586 & 5.583 & 4.0913 \mathrm{E}-09 \\ 777.30 & 21.5746 & 4.077 & 1.9872 \mathrm{E}-09\end{array}$




\begin{tabular}{crrr}
$\begin{array}{c}\text { Temperature } \\
(\mathrm{K})\end{array}$ & $\begin{array}{c}\text { Pressure } \\
\text { (MPa) }\end{array}$ & $\begin{array}{c}\text { Density } \\
\text { (mol/L) }\end{array}$ & $\begin{array}{c}\text { Solubility } \\
\text { (mole fraction) }\end{array}$ \\
\hline 778.24 & 21.5746 & 4.067 & $4.6757 \mathrm{E}-09$ \\
783.61 & 27.4586 & 5.457 & $4.0913 \mathrm{E}-09$ \\
789.68 & 25.4973 & 4.866 & $1.2391 \mathrm{E}-08$ \\
790.38 & 23.5360 & 4.389 & $5.1433 \mathrm{E}-09$ \\
792.24 & 25.4973 & 4.831 & $1.5898 \mathrm{E}-08$ \\
793.88 & 25.4973 & 4.809 & $2.6886 \mathrm{E}-09$ \\
795.28 & 21.5746 & 3.896 & $1.9872 \mathrm{E}-09$ \\
795.51 & 23.5360 & 4.331 & $5.3771 \mathrm{E}-09$ \\
801.58 & 29.4200 & 5.654 & $4.6757 \mathrm{E}-09$ \\
802.05 & 29.4200 & 5.646 & $5.4940 \mathrm{E}-09$ \\
803.22 & 23.5360 & 4.248 & $4.4420 \mathrm{E}-09$ \\
804.62 & 27.4586 & 5.129 & $6.3123 \mathrm{E}-09$ \\
807.42 & 21.5746 & 3.787 & $2.2210 \mathrm{E}-09$ \\
810.45 & 25.4973 & 4.603 & $5.6109 \mathrm{E}-09$ \\
812.09 & 25.4973 & 4.585 & $6.5460 \mathrm{E}-09$ \\
815.82 & 21.5746 & 3.717 & $3.2730 \mathrm{E}-09$ \\
816.75 & 25.4973 & 4.532 & $5.6109 \mathrm{E}-09$ \\
820.49 & 23.5360 & 4.079 & $3.3899 \mathrm{E}-09$ \\
822.36 & 23.5360 & 4.061 & $2.8054 \mathrm{E}-09$ \\
841.50 & 21.5746 & 3.524 & $4.6757 \mathrm{E}-09$ \\
849.43 & 27.4586 & 4.596 & $5.8447 \mathrm{E}-09$ \\
852.24 & 25.4973 & 4.186 & $6.8967 \mathrm{E}-09$ \\
854.34 & 25.4973 & 4.168 & $6.3123 \mathrm{E}-09$ \\
854.57 & 27.4586 & 4.545 & $7.0136 \mathrm{E}-09$ \\
858.07 & 25.4973 & 4.136 & $6.8967 \mathrm{E}-09$ \\
863.21 & 29.4200 & 4.841 & $6.8967 \mathrm{E}-09$ \\
865.77 & 21.5746 & 3.366 & $7.9488 \mathrm{E}-09$ \\
866.71 & 23.5360 & 3.709 & $6.6629 \mathrm{E}-09$ \\
868.11 & 23.5360 & 3.699 & $7.4812 \mathrm{E}-09$ \\
869.27 & 21.5746 & 3.345 & $6.5460 \mathrm{E}-09$ \\
871.38 & 29.4200 & 4.758 & $7.8319 \mathrm{E}-09$ \\
898.92 & 25.4973 & 3.830 & $8.1826 \mathrm{E}-09$ \\
908.49 & 25.4973 & 3.768 & $7.9488 \mathrm{E}-09$ \\
929.50 & 2.9420 & 0.385 & $9.5853 \mathrm{E}-09$ \\
948.41 & 10.3950 & 1.365 & $9.3515 \mathrm{E}-09$ \\
950.04 & 2.9420 & 0.376 & $1.0871 \mathrm{E}-08$ \\
973.38 & 9.8066 & 1.248 & $1.6014 \mathrm{E}-08$ \\
\hline & & & \\
\hline
\end{tabular}

Reference: Sastry, V., 1957, Die Loslichkeit von Salzen in Hochgespanntem Wasserdampf und die I1 Klarung der Frage der Turbinenversalzung: Doctoral Dissertation - Technische Hochschule Karlsruhe

Units of original measurements: Temperature in degrees $C$, Pressure in $\mathrm{kg} / \mathrm{cm} 2$, Solubility in $\mathrm{mg} / \mathrm{kg}$

\begin{tabular}{crrc}
$\begin{array}{c}\text { Temperature } \\
(\mathrm{K})\end{array}$ & $\begin{array}{c}\text { Pressure } \\
\text { (MPa) }\end{array}$ & $\begin{array}{c}\text { Density } \\
\text { (mol/L) }\end{array}$ & $\begin{array}{c}\text { Solubility } \\
\text { (mole fraction) }\end{array}$ \\
\hline 675.15 & 8.0414 & 1.618 & $4.0586 \mathrm{E}-08$ \\
675.15 & 9.2182 & 1.896 & $4.0586 \mathrm{E}-08$ \\
675.15 & 9.6105 & 1.992 & $4.9464 \mathrm{E}-08$ \\
675.15 & 10.1989 & 2.138 & $4.0586 \mathrm{E}-08$ \\
675.15 & 10.4931 & 2.213 & $4.5659 \mathrm{E}-08$ \\
675.15 & 11.2777 & 2.418 & $3.9317 \mathrm{E}-08$
\end{tabular}

Temperature Pressure Density Solubility

\begin{tabular}{llrr}
$(\mathrm{K})$ & $(\mathrm{MPa})$ & $(\mathrm{mol} / \mathrm{L})$ & $($ mole fraction $)$ \\
\hline 675.15 & 11.2777 & 2.418 & $3.5512 \mathrm{E}-08$ \\
675.15 & 12.1603 & 2.658 & $3.9317 \mathrm{E}-08$ \\
675.15 & 12.3564 & 2.713 & $2.5366 \mathrm{E}-08$ \\
675.15 & 12.9448 & 2.881 & $4.5659 \mathrm{E}-08$ \\
675.15 & 13.4351 & 3.025 & $3.6781 \mathrm{E}-08$ \\
675.15 & 13.6312 & 3.084 & $5.2000 \mathrm{E}-08$ \\
675.15 & 14.2196 & 3.264 & $4.0586 \mathrm{E}-08$ \\
675.15 & 14.4158 & 3.326 & $5.2000 \mathrm{E}-08$ \\
675.15 & 15.0042 & 3.515 & $5.3268 \mathrm{E}-08$ \\
675.15 & 15.2003 & 3.580 & $7.1025 \mathrm{E}-08$ \\
675.15 & 15.9848 & 3.847 & $9.0049 \mathrm{E}-08$ \\
675.15 & 15.9848 & 3.847 & $6.2147 \mathrm{E}-08$ \\
675.15 & 16.6713 & 4.093 & $1.1034 \mathrm{E}-07$ \\
675.15 & 16.7694 & 4.130 & $9.8927 \mathrm{E}-08$ \\
\hline
\end{tabular}

Reference: Morey, G.W. and Hesselgesser, J.M., 1951, The 22 solubility of some minerals in superheated steam 22 at high pressures: Economic Geology, v. 46, p. 821-835

Units of original measurements: Temperature in degrees $C$, Pressure in psia, Solubility in wt\%

Temperature Pressure Density Solubility

\begin{tabular}{crrc}
$(\mathrm{K})$ & \multicolumn{1}{c}{$(\mathrm{MPa})$} & $(\mathrm{mol} / \mathrm{L})$ & $($ mole fraction $)$ \\
\hline 773.15 & 6.7000 & 1.100 & $1.0883 \mathrm{E}-06$ \\
773.15 & 13.3000 & 2.323 & $4.7036 \mathrm{E}-06$ \\
773.15 & 66.6000 & 21.376 & $3.1579 \mathrm{E}-05$ \\
773.15 & 100.0000 & 29.324 & $5.4828 \mathrm{E}-04$ \\
\hline
\end{tabular}

Reference: Novikov, B.E., 1973, Investigation of 30 thermodynamic properties and behavior of salts 30 with common ions in boiler water at high temperatures: Dissertation - Moscow Power Institute

Units of original measurements: Temperature in degrees $C$, Pressure in $\mathrm{kgf} / \mathrm{cm} 2$, Solubility in $\mathrm{mg} / \mathrm{kg}$

Temperature Pressure Density Solubility

\begin{tabular}{crrr}
\multicolumn{1}{c}{$(\mathrm{K})$} & \multicolumn{1}{c}{$(\mathrm{MPa})$} & $(\mathrm{mol} / \mathrm{L})$ & (mole fraction) \\
\hline 486.65 & 1.8142 & 0.497 & $3.0693 \mathrm{E}-18$ \\
506.15 & 2.8243 & 0.777 & $8.7386 \mathrm{E}-17$ \\
519.15 & 3.4814 & 0.953 & $7.1659 \mathrm{E}-16$ \\
527.15 & 4.0207 & 1.105 & $2.5366 \mathrm{E}-15$ \\
535.15 & 4.6386 & 1.284 & $8.7766 \mathrm{E}-15$ \\
541.90 & 5.1975 & 1.449 & $2.4859 \mathrm{E}-14$ \\
555.65 & 6.5214 & 1.859 & $1.9785 \mathrm{E}-13$ \\
570.15 & 8.0905 & 2.371 & $1.6234 \mathrm{E}-12$ \\
582.65 & 9.7086 & 2.953 & $9.6391 \mathrm{E}-12$ \\
594.15 & 11.1796 & 3.488 & $4.6673 \mathrm{E}-11$ \\
599.15 & 11.8661 & 3.749 & $9.2078 \mathrm{E}-11$ \\
609.15 & 13.4351 & 4.409 & $3.8429 \mathrm{E}-10$ \\
617.65 & 15.2003 & 5.367 & $1.3190 \mathrm{E}-09$ \\
632.65 & 18.4365 & 7.636 & $1.9912 \mathrm{E}-08$ \\
640.65 & 20.4959 & 10.053 & $2.0039 \mathrm{E}-07$ \\
\hline
\end{tabular}


$\begin{array}{cl}\text { Reference: } & \text { Ravich, M.I. and Borovaya, F.E., 1964, Phase } \\ \text { equilibria in the sodium sulphate-water system at } \\ \text { high temperatures and pressures: Russian } \\ \text { Journal of Inorganic Chemistry, v. } 9, \text { n. 4, p. 520- } \\ 532\end{array}$

Units of original measurements: Temperature in degrees $C$,

Pressure in $\mathrm{kgf} / \mathrm{cm} 2$, Solubility in wt\%, " indicates data point is at saturation

\begin{tabular}{crcc}
$\begin{array}{c}\text { Temperature } \\
\text { (K) }\end{array}$ & $\begin{array}{c}\text { Pressure } \\
\text { (MPa) }\end{array}$ & $\begin{array}{c}\text { Density } \\
\text { (mol/L) }\end{array}$ & $\begin{array}{c}\text { Solubility } \\
\text { (mole fraction) }\end{array}$ \\
\hline 593.15 & 11.0815 & 37.029 & $1.7001 \mathrm{E}-02{ }^{*}$ \\
593.15 & 29.4200 & 39.548 & $3.5514 \mathrm{E}-02$ \\
593.15 & 39.2266 & 40.507 & $4.5900 \mathrm{E}-02$ \\
593.15 & 71.5885 & 42.862 & $6.3927 \mathrm{E}-02$ \\
593.15 & 95.4187 & 44.169 & $7.2128 \mathrm{E}-02$ \\
593.15 & 121.9947 & 45.390 & $7.9469 \mathrm{E}-02$ \\
623.15 & 16.4752 & 31.901 & $3.2415 \mathrm{E}-03$ \\
623.15 & 27.3606 & 35.233 & $9.4560 \mathrm{E}-03$ \\
623.15 & 40.0111 & 37.295 & $2.0229 \mathrm{E}-02$ \\
623.15 & 50.7985 & 38.566 & $3.3571 \mathrm{E}-02$ \\
623.15 & 50.9946 & 38.586 & $3.4537 \mathrm{E}-02$ \\
623.15 & 55.1134 & 39.002 & $3.9530 \mathrm{E}-02$ \\
623.15 & 58.8399 & 39.354 & $4.4808 \mathrm{E}-02$ \\
623.15 & 66.1949 & 39.994 & $5.3910 \mathrm{E}-02$ \\
623.15 & 75.8054 & 40.742 & $6.1329 \mathrm{E}-02$ \\
623.15 & 97.2820 & 42.156 & $7.0716 \mathrm{E}-02$ \\
623.15 & 119.9353 & 43.391 & $7.9469 \mathrm{E}-02$ \\
643.15 & 20.9862 & 25.058 & $6.3693 \mathrm{E}-04$ \\
643.15 & 38.7363 & 34.346 & $6.6310 \mathrm{E}-03$ \\
643.15 & 48.5429 & 36.004 & $1.3139 \mathrm{E}-02$ \\
643.15 & 58.3496 & 37.269 & $2.2736 \mathrm{E}-02$ \\
643.15 & 68.6466 & 38.354 & $3.4537 \mathrm{E}-02$ \\
643.15 & 78.4532 & 39.236 & $5.0396 \mathrm{E}-02$ \\
653.15 & 29.4200 & 29.326 & $1.2795 \mathrm{E}-03$ \\
653.15 & 39.2266 & 32.816 & $3.5736 \mathrm{E}-03$ \\
653.15 & 49.0333 & 34.787 & $7.3275 \mathrm{E}-03$ \\
653.15 & 58.8399 & 36.222 & $1.3896 \mathrm{E}-02$ \\
653.15 & 78.4532 & 38.339 & $4.0044 \mathrm{E}-02$ \\
673.15 & 49.0333 & 31.845 & $2.5817 \mathrm{E}-03$ \\
673.15 & 58.8399 & 33.802 & $4.5790 \mathrm{E}-03$ \\
673.15 & 64.2336 & 34.648 & $6.6310 \mathrm{E}-03$ \\
673.15 & 69.3330 & 35.351 & $9.8166 \mathrm{E}-03$ \\
673.15 & 76.0015 & 36.165 & $1.3896 \mathrm{E}-02$ \\
673.15 & 78.4532 & 36.440 & $1.6213 \mathrm{E}-02$ \\
673.15 & 81.3952 & 36.755 & $2.1892 \mathrm{E}-02$ \\
673.15 & 88.5541 & 37.464 & $3.6501 \mathrm{E}-02$ \\
673.15 & 95.1245 & 38.055 & $5.3910 \mathrm{E}-02$ \\
673.15 & 98.4588 & 38.337 & $7.0716 \mathrm{E}-02$ \\
673.15 & 115.7185 & 39.640 & $8.6513 \mathrm{E}-02$ \\
703.15 & 98.5568 & 35.754 & $1.3896 \mathrm{E}-02$ \\
703.15 & 104.9312 & 36.381 & $2.1892 \mathrm{E}-02$ \\
703.15 & 106.8925 & 36.564 & $3.0733 \mathrm{E}-02$ \\
703.15 & 107.8732 & 36.654 & $4.0562 \mathrm{E}-02$ \\
703.15 & 108.3635 & 36.698 & $5.1553 \mathrm{E}-02$
\end{tabular}

Temperature Pressure Density Solubility (K) (MPa) (mol/L) (mole fraction)

\begin{tabular}{crcc}
\multicolumn{1}{c}{$(\mathrm{K})$} & \multicolumn{1}{c}{$(\mathrm{MPa})$} & $(\mathrm{mol} / \mathrm{L})$ & (mole fraction) \\
\hline 703.15 & 109.8345 & 36.830 & $6.3927 \mathrm{E}-02$ \\
703.15 & 110.3248 & 36.873 & $7.8261 \mathrm{E}-02$ \\
703.15 & 110.8152 & 36.916 & $8.8303 \mathrm{E}-02$ \\
723.15 & 78.4532 & 31.023 & $1.2795 \mathrm{E}-03$ \\
723.15 & 88.5541 & 32.624 & $2.8449 \mathrm{E}-03$ \\
723.15 & 106.8925 & 34.852 & $1.2016 \mathrm{E}-02$ \\
773.15 & 122.9754 & 32.378 & $2.5817 \mathrm{E}-03$ \\
\hline
\end{tabular}

Reference: Schroeder, W.C., Gabriel, A. and Partridge, E.P., 1935, Solubility equilibria of sodium sulfate 32 at temperatures of 150 to 350 degrees. I. Effect of sodium hydroxide and sodium chloride: Journal of the American Chemical Society, v. 57, p. $1539-1546$

Units of original measurements: Temperature in degrees $C$,

Solubility in wt\%, all data points are at saturation

Temperature Pressure Density Solubility (K) (MPa) (mol/L) (mole fraction)

413.15

413.15

423.15

429.15

433.15

433.15

443.15

443.15

453.15

453.15

453.15

453.15

463.15

463.15

463.15

463.15

477.15

483.15

483.15

492.15

492.15

494.15

494.15

503.15

503.15

513.15

514.15

514.15

523.15

524.15

524.15

533.15

533.15

533.15

543.15

$\begin{array}{lll}0.3615 & 51.408 & 5.0346 \mathrm{E}-02\end{array}$

$0.3615 \quad 51.408 \quad 5.0574 \mathrm{E}-02$

$0.4762 \quad 50.902 \quad 5.0689 \mathrm{E}-02$

$0.5578 \quad 50.586 \quad 5.1260 \mathrm{E}-02$

$0.6182 \quad 50.371 \quad 5.0574 \mathrm{E}-02$

$0.6182 \quad 50.371 \quad 5.1146 \mathrm{E}-02$

$0.7922 \quad 49.816 \quad 5.1374 \mathrm{E}-02$

$0.7922 \quad 49.816 \quad 5.1830 \mathrm{E}-02$

$1.0028 \quad 49.236 \quad 5.2058 \mathrm{E}-02$

$1.0028 \quad 49.236 \quad 5.2742 \mathrm{E}-02$

$1.0028 \quad 49.236 \quad 5.1716 \mathrm{E}-02$

$1.0028 \quad 49.236 \quad 5.2058 \mathrm{E}-02$

$1.2552 \quad 48.630 \quad 5.3083 \mathrm{E}-02$

$1.2552 \quad 48.630 \quad 5.3424 \mathrm{E}-02$

$1.2552 \quad 48.630 \quad 5.2742 \mathrm{E}-02$

$1.2552 \quad 48.630 \quad 5.2969 \mathrm{E}-02$

$1.6893 \quad 47.734 \quad 5.1716 \mathrm{E}-02$

$1.9077 \quad 47.333 \quad 5.3765 \mathrm{E}-02$

$1.9077 \quad 47.333 \quad 5.3992 \mathrm{E}-02$

$2.2756 \quad 46.710 \quad 5.3878 \mathrm{E}-02$

$2.3643 \quad 46.568 \quad 5.4672 \mathrm{E}-02$

$2.3643 \quad 46.568 \quad 5.4219 \mathrm{E}-02$

$2.7971 \quad 45.912 \quad 5.5465 \mathrm{E}-02$

$2.7971 \quad 45.912 \quad 5.4559 \mathrm{E}-02$

$3.3469 \quad 45.149 \quad 5.5012 \mathrm{E}-02$

$3.4062 \quad 45.070 \quad 5.4786 \mathrm{E}-02$

$3.4062 \quad 45.070 \quad 5.4559 \mathrm{E}-02$

$3.9762 \quad 44.345 \quad 5.1716 \mathrm{E}-02$

$4.0438 \quad 44.263 \quad 5.3651 \mathrm{E}-02$

$4.0438 \quad 44.263 \quad 5.3424 \mathrm{E}-02$

$4.6923 \quad 43.498 \quad 5.0689 \mathrm{E}-02$

$4.6923 \quad 43.498 \quad 5.0117 \mathrm{E}-02$

$4.6923 \quad 43.498 \quad 4.9888 \mathrm{E}-02$

$5.5030 \quad 42.601 \quad 4.7017 E-02$
$2.2756 \quad 46.710 \quad 5.3992 \mathrm{E}-02$ 
Temperature Pressure Density Solubility

\begin{tabular}{crcc}
$(\mathrm{K})$ & \multicolumn{1}{c}{$(\mathrm{MPa})$} & $(\mathrm{mol} / \mathrm{L})$ & $($ mole fraction $)$ \\
\hline 543.15 & 5.5030 & 42.601 & $4.9201 \mathrm{E}-02$ \\
552.15 & 6.3204 & 41.745 & $4.2620 \mathrm{E}-02$ \\
552.15 & 6.3204 & 41.745 & $4.3433 \mathrm{E}-02$ \\
554.15 & 6.5139 & 41.548 & $4.1689 \mathrm{E}-02$ \\
561.15 & 7.2274 & 40.837 & $3.8651 \mathrm{E}-02$ \\
572.15 & 8.4676 & 39.643 & $2.9540 \mathrm{E}-02$ \\
574.15 & 8.7096 & 39.415 & $3.1328 \mathrm{E}-02$ \\
574.15 & 8.7096 & 39.415 & $2.7745 \mathrm{E}-02$ \\
583.15 & 9.8651 & 38.338 & $2.2804 \mathrm{E}-02$ \\
593.15 & 11.2843 & 37.029 & $1.6220 \mathrm{E}-02$ \\
603.15 & 12.8581 & 35.568 & $9.1736 \mathrm{E}-03$ \\
613.15 & 14.6007 & 33.897 & $5.2986 \mathrm{E}-03$ \\
622.15 & 16.3276 & 32.121 & $3.0347 \mathrm{E}-03$ \\
623.15 & 16.5294 & 31.901 & $3.0347 \mathrm{E}-03$ \\
\hline
\end{tabular}

Reference: Booth, H.S. and Bidwell, R.M., 1950, Solubilities of salts in water at high temperatures: Journal of the American Chemical Society, v. 72, p. 2567 2575

Units of original measurements: Temperature in degrees $C$, Solubility in wt\%, " indicates data point is at saturation

Temperature Pressure Density Solubility

\begin{tabular}{clcc}
$(\mathrm{K})$ & $(\mathrm{MPa})$ & $(\mathrm{mol} / \mathrm{L})$ & (mole fraction) \\
\hline 603.15 & 12.8581 & 35.568 & $7.6641 \mathrm{E}-03^{*}$ \\
627.15 & 17.3577 & 30.958 & $2.1233 \mathrm{E}-03^{*}$ \\
633.15 & 18.6660 & 29.286 & $1.1505 \mathrm{E}-03^{*}$ \\
655.15 & 12.7860 & 24.030 & $4.7973 \mathrm{E}-04$ \\
\hline
\end{tabular}

Reference: Khaibullin, I.Kh. and Novikov, B.E., 1973, A

34 thermodynamic study of aqueous and steam solutions of sodium sulfate at high temperatures: Teplofizika Vysokikh Temperatur, v. 11, n. 2, p. 320-327

Units of original measurements: Temperature in degrees $C$, Pressure in $\mathrm{kg} / \mathrm{cm} 2$, Solubility in $\mathrm{wt} \%$, all data points are at saturation

\begin{tabular}{crcc}
$\begin{array}{c}\text { Temperature } \\
(\mathrm{K})\end{array}$ & $\begin{array}{c}\text { Pressure } \\
\text { (MPa) }\end{array}$ & $\begin{array}{c}\text { Density } \\
(\mathrm{mol} / \mathrm{L})\end{array}$ & $\begin{array}{c}\text { Solubility } \\
\text { (mole fraction) }\end{array}$ \\
\hline 527.15 & 4.0207 & 44.012 & $5.1553 \mathrm{E}-02$ \\
541.95 & 5.1975 & 42.711 & $4.7004 \mathrm{E}-02$ \\
555.65 & 6.5214 & 41.398 & $4.0562 \mathrm{E}-02$ \\
570.15 & 8.0905 & 39.868 & $3.0733 \mathrm{E}-02$ \\
582.65 & 9.7086 & 38.400 & $2.1892 \mathrm{E}-02$ \\
594.15 & 11.1796 & 36.891 & $1.3896 \mathrm{E}-02$ \\
599.15 & 11.8661 & 36.174 & $1.0908 \mathrm{E}-02$ \\
609.15 & 13.4351 & 34.596 & $6.6310 \mathrm{E}-03$ \\
617.65 & 15.2003 & 33.050 & $3.9072 \mathrm{E}-03$ \\
632.65 & 18.4365 & 29.441 & $1.2795 \mathrm{E}-03$ \\
640.65 & 20.3978 & 26.426 & $6.3693 \mathrm{E}-04$ \\
\hline
\end{tabular}

Reference: Khaibullin, I.Kh. and Novikov, B.E., 1973, Gamma-ray examination of saturation parameters and thermophysical properties of multicomponent salt systems at high temperatures: Mater. Vses. Nauchno-Tekh. Soveshch. Teploobmennym Teplofiz. Svoistvam Morsk. Solonovatykh Vod Ikh Ispol'z, p. 339-350

Units of original measurements: Temperature in degrees $C$, Solubility in mol/L, all data points are at saturation

\begin{tabular}{cccc}
$\begin{array}{c}\text { Temperature } \\
(\mathrm{K})\end{array}$ & $\begin{array}{c}\text { Pressure } \\
(\mathrm{MPa})\end{array}$ & $\begin{array}{c}\text { Density } \\
(\mathrm{mol} / \mathrm{L})\end{array}$ & $\begin{array}{c}\text { Solubility } \\
\text { (mole fraction) }\end{array}$ \\
\hline 583.15 & 9.8651 & 38.338 & $2.3893 \mathrm{E}-02$ \\
593.15 & 11.2843 & 37.029 & $1.5717 \mathrm{E}-02$ \\
603.15 & 12.8581 & 35.568 & $9.8402 \mathrm{E}-03$ \\
613.15 & 14.6007 & 33.897 & $5.7232 \mathrm{E}-03$ \\
623.15 & 16.5294 & 31.901 & $2.9278 \mathrm{E}-03$ \\
633.15 & 18.6660 & 29.286 & $1.2668 \mathrm{E}-03$ \\
\hline
\end{tabular}





\section{APPENDIX B - VAPOR PRESSURES}

The vapor pressure of a solute can provide a lower bound (though usually far too low for the systems and conditions of interest in this report) on its solubility. In addition, it may be a useful quantity in future modeling efforts. This appendix therefore discusses the vapor pressure of compounds of interest in this report, and tabulates them where appropriate.

Vapor pressures for pure condensed phases can be computed from the free energy functions given in thermochemical tables such as the JANAF [B1] compilation. In order to make this computation, there must be a table both for the condensed phase of interest and for the gas phase (the table is normally for the ideal gas at $0.1 \mathrm{MPa}$; computing the vapor pressure from these tables requires assuming ideality in the vapor phase which is generally a good assumption at these low pressures). For the compounds of interest in this study, the measured vapor pressures are almost always at higher temperatures than those of interest. Those measurements are used to tie the gas tables to those for the condensed phase at that particular temperature, but then the accuracy of a vapor pressure computation at a lower temperature depends on the accuracy of the heat capacities used to take the free energy function to lower temperatures. Fortunately, common salts and minerals are sufficiently well studied that extrapolation with heat capacity data does not introduce a great deal of error.

The following sections describe the individual condensed phases of interest.

\section{$\mathrm{NaCl}$}

Fairly consistent vapor pressure measurements exist down to $743 \mathrm{~K}$. The JANAF tables allow calculations throughout our temperature range. A complication is the extensive dimerization in the vapor. The JANAF tables have separate ideal-gas tables for the monomer and dimer. These can be used to compute separate monomer and dimer vapor pressures. The points above $1073 \mathrm{~K}$ are for the vapor pressure above molten $\mathrm{NaCl}$.

Table B-1. $\mathrm{NaCl}$ vapor pressures from the JANAF tables [B1].

\begin{tabular}{cll}
\hline $\mathrm{T}(\mathrm{K})$ & $\mathrm{P}^{\text {monomer }}(\mathrm{MPa})$ & $\mathrm{P}^{\text {dimer }}(\mathrm{MPa})$ \\
\hline 400 & $1.62 \times 10^{-23}$ & $9.32 \times 10^{-26}$ \\
500 & $1.41 \times 10^{-17}$ & $3.70 \times 10^{-19}$ \\
600 & $1.20 \times 10^{-13}$ & $8.33 \times 10^{-15}$ \\
700 & $7.30 \times 10^{-11}$ & $9.86 \times 10^{-12}$ \\
800 & $8.57 \times 10^{-9}$ & $1.86 \times 10^{-9}$ \\
900 & $3.37 \times 10^{-7}$ & $1.03 \times 10^{-7}$ \\
1000 & $6.15 \times 10^{-6}$ & $2.41 \times 10^{-6}$ \\
1100 & $5.96 \times 10^{-5}$ & $2.61 \times 10^{-5}$ \\
1200 & $3.17 \times 10^{-4}$ & $1.22 \times 10^{-4}$ \\
\hline
\end{tabular}


$\mathrm{SiO}_{2}$

Vapor pressure measurements are fairly consistent, but are all above $1600 \mathrm{~K}$. The JANAF tables allow extrapolation to lower temperatures; the system has been studied enough that such extrapolation is probably fairly good. There has been some reexamination of the heat capacity data since the publication of the JANAF tables, but the differences are too small to worry about given the uncertainties in the vapor pressures.

Table B-2. $\mathrm{SiO}_{2}$ vapor pressures from the JANAF tables [B1].

\begin{tabular}{cl}
\hline $\mathrm{T}(\mathrm{K})$ & $\mathrm{P}^{\text {sat }}(\mathrm{MPa})$ \\
\hline 400 & $5.38 \times 10^{-71}$ \\
500 & $3.36 \times 10^{-55}$ \\
600 & $1.10 \times 10^{-44}$ \\
700 & $3.46 \times 10^{-37}$ \\
800 & $1.41 \times 10^{-31}$ \\
900 & $3.16 \times 10^{-27}$ \\
1000 & $9.33 \times 10^{-24}$ \\
1100 & $6.37 \times 10^{-21}$ \\
1200 & $1.45 \times 10^{-18}$ \\
1300 & $1.42 \times 10^{-16}$ \\
1400 & $7.19 \times 10^{-15}$ \\
1500 & $2.14 \times 10^{-13}$ \\
\hline
\end{tabular}

$\mathrm{Na}_{2} \mathrm{SO}_{4}$

Scattered vapor pressure data exist at higher temperatures (above about $1100 \mathrm{~K}$ ). These measurements are complicated by a dissociation reaction that is important at high temperatures (but relatively small compared to the uncertainty of the measurements in the region of interest here). These data are input to the JANAF compilation, which allows reasonable estimates of vapor pressure throughout the temperature range of interest here.

Following is a table of vapor pressures generated from the JANAF tables. All the points are for crystalline sodium sulfate. 
Table B-3. $\mathrm{Na}_{2} \mathrm{SO}_{4}$ vapor pressures from the JANAF tables [B1].

\begin{tabular}{cl}
\hline $\mathrm{T}(\mathrm{K})$ & $\mathrm{P}^{\text {sat }}(\mathrm{MPa})$ \\
\hline 400 & $1.99 \times 10^{-37}$ \\
500 & $1.55 \times 10^{-28}$ \\
600 & $1.11 \times 10^{-22}$ \\
700 & $1.51 \times 10^{-18}$ \\
800 & $1.75 \times 10^{-15}$ \\
900 & $3.93 \times 10^{-13}$ \\
1000 & $2.81 \times 10^{-11}$ \\
1100 & $8.75 \times 10^{-10}$ \\
\hline
\end{tabular}

\section{$\mathrm{NaOH}$}

Old vapor pressure data exist at very high temperatures. The JANAF tables use these and other means to compile sufficient information for vapor pressure calculation. This information has been updated since the JANAF publication; calculations here are based on new tables from recent work [B2]. The results are again complicated by dimerization. There is also a solid-solid phase transition at $568 \mathrm{~K}$ and a solid-liquid transition at $594 \mathrm{~K}$.

Table B-4. NaOH vapor pressures from Gurvich et al. [B2].

\begin{tabular}{ccc}
\hline $\mathrm{T}(\mathrm{K})$ & $\mathrm{P}^{\text {monomer }}(\mathrm{MPa})$ & $\mathrm{P}^{\text {dimer }}(\mathrm{MPa})$ \\
\hline 400 & $8.08 \times 10^{-24}$ & $4.80 \times 10^{-22}$ \\
500 & $9.66 \times 10^{-18}$ & $3.39 \times 10^{-16}$ \\
568 & $7.40 \times 10^{-15}$ & $1.93 \times 10^{-13}$ \\
594 & $5.86 \times 10^{-14}$ & $1.30 \times 10^{-12}$ \\
600 & $9.08 \times 10^{-14}$ & $1.91 \times 10^{-12}$ \\
700 & $4.21 \times 10^{-11}$ & $4.22 \times 10^{-10}$ \\
800 & $3.92 \times 10^{-9}$ & $2.13 \times 10^{-8}$ \\
900 & $1.26 \times 10^{-7}$ & $4.10 \times 10^{-7}$ \\
1000 & $1.95 \times 10^{-6}$ & $4.06 \times 10^{-6}$ \\
1100 & $1.78 \times 10^{-5}$ & $2.51 \times 10^{-5}$ \\
1200 & $1.09 \times 10^{-4}$ & $1.09 \times 10^{-4}$ \\
\hline
\end{tabular}

$\mathrm{Fe}_{3} \mathrm{O}_{4}$ and $\mathrm{Fe}_{2} \mathrm{O}_{3}$

No information available. 
$\mathrm{Na}_{3} \mathrm{PO}_{4}$

No information available.

$\mathrm{CuO}$

Vapor pressure can be calculated from the JANAF tables. Their calculations are indirect, as no reliable vapor pressure measurements exist. It was not felt worthwhile to pursue it at this point.

$\mathrm{Cu}_{2} \mathrm{O}$

No information available.

\section{References for Appendix B}

[B1] Chase, M.W., Davies, C.A., Downey, J.R., Frurip, D.J., McDonald, R.A., and Syverud, A.N., JANAF Thermochemical Tables, 3rd ed., J. Phys. Chem. Ref. Data, 14, Supplement 1 (1985).

[B2] Gurvich, L.V., Bergman, G.A., Gorokhov, L.N., Iorish, V.S., Leonidov, V.Ya., and Yungman, V.S., Thermodynamic Properties of Alkali Metal Hydroxides Part 1. Lithium and Sodium Hydroxides, J. Phys. Chem. Ref. Data, 25, 1211 (1996). 
APPENDIX C - VAPOR-LIQUID PARTITIONING FOR $\mathrm{Na}_{3} \mathrm{PO}_{4}$ and $\mathrm{Na}_{2} \mathrm{SO}_{4}$

Table C-1. Data of Goodstine [29] for partitioning of $\mathrm{Na}_{3} \mathrm{PO}_{4}$ between water and steam.

\begin{tabular}{|c|c|c|c|c|c|c|c|}
\hline \multirow{3}{*}{$\begin{array}{c}\text { Pressure } \\
\text { (psig) }\end{array}$} & \multicolumn{2}{|c|}{ Concentration } & \multicolumn{2}{|c|}{ Concentration } & \multicolumn{2}{|c|}{ Concentration } & \multirow{3}{*}{$\begin{array}{c}\text { Partition } \\
\text { Coefficient } \\
(-) \\
\end{array}$} \\
\hline & Water & Steam & & & & & \\
\hline & \multicolumn{2}{|c|}{ (ppm of $\mathrm{Na})$} & \multicolumn{2}{|c|}{ (ppm of $\mathrm{Na}_{3} \mathrm{PO}_{4}$ ) } & (mole fraction & & \\
\hline 3080 & $8.640 E+00$ & $9.297 E-02$ & $2.054 \mathrm{E}+01$ & $2.210 \mathrm{E}-01$ & $2.257 \mathrm{E}-06$ & $2.428 \mathrm{E}-08$ & $1.076 \mathrm{E}-02$ \\
\hline 3080 & $1.459 E+01$ & 1.999E-01 & $3.468 E+01$ & $4.751 \mathrm{E}-01$ & $3.811 \mathrm{E}-06$ & 5.221E-08 & 1.370E-02 \\
\hline 3080 & $.077 E+01$ & 3.330E-01 & $4.937 E+01$ & 7.915E-01 & 5.426E-06 & 8.698E-08 & $1.603 E-02$ \\
\hline 3080 & $.030 E+01$ & $4.512 \mathrm{E}-01$ & $7.203 E+01$ & $1.072 E+00$ & 7.916E-06 & $1.179 \mathrm{E}-07$ & $1.489 E-02$ \\
\hline 3000 & $5.995 E+00$ & & $1.425 E+01$ & & & & \\
\hline 3000 & $183 E+00$ & 5.513E-02 & $2.183 E+01$ & $1.311 \mathrm{E}-01$ & 2.399E-06 & 08 & $6.004 \mathrm{E}-03$ \\
\hline 3000 & $589 E+01$ & 8.964E-02 & $3.776 \mathrm{E}+01$ & 2.131E-01 & 4.150E-06 & 2.341 & 5.642E-03 \\
\hline 3000 & $978 \mathrm{E}+01$ & $1.322 \mathrm{E}-01$ & $4.702 E+01$ & 3.143E-01 & 5.168E-06 & 3.454 & $6.684 \mathrm{E}-03$ \\
\hline 3000 & $154 E+01$ & 1.405E-01 & $5.121 E+01$ & 3.340E-01 & 5.628E-06 & $3.671 \mathrm{l}$ & $6.522 E$ \\
\hline 3000 & $957 E+01$ & $1.626 \mathrm{E}-01$ & $7.030 E+01$ & 3.865E-01 & 7.725E-06 & $4.247 \mathrm{E}$ & 5.497E-03 \\
\hline 3000 & $342 E+01$ & 4.350E-01 & $1.103 E+02$ & $1.034 E+00$ & $1.213 E-05$ & 1.136 & $9.372 \mathrm{E}-03$ \\
\hline 3000 & $922 E+01$ & 7.336E-01 & $1.408 \mathrm{E}+02$ & $1.744 \mathrm{E}+00$ & 1.547E-05 & 1.916 & $1.238 \mathrm{E}-02$ \\
\hline 3000 & $.063 E+02$ & $1.267 E+00$ & $2.526 E+02$ & $3.013 E+00$ & 2.777E-05 & $3.310 \mathrm{E}$ & 1.192E-02 \\
\hline 3000 & $730 E+02$ & $2.086 E+00$ & $4.113 E+02$ & $4.958 E+00$ & $4.521 \mathrm{E}-05$ & $5.448 \mathrm{E}$ & 1.205E-02 \\
\hline 3000 & $134 E+02$ & $3.115 E+00$ & $5.785 E+02$ & $7.403 E+00$ & & 8.136 & \\
\hline 2800 & $.933 E+00$ & $3.021 E-03$ & $1.173 E+01$ & 7.182E-03 & $1.289 \mathrm{E}-06$ & $7.892 E-10$ & \\
\hline 2800 & $35 E+00$ & $3.670 \mathrm{E}-03$ & $2.029 E+01$ & 8.723E-03 & & 9.586 & \\
\hline 2800 & $003 E+01$ & 1.987E-02 & $4.760 E+01$ & 4.723E-02 & 5.231E-06 & & $9.921 E-04$ \\
\hline 2800 & $290 E+01$ & 2.271E-02 & $5.443 E+01$ & 5.398E-02 & 5.981E-06 & 5.932 & 9.917 E-04 \\
\hline 2800 & $.922 E+01$ & 3.310E-02 & $6.944 E+01$ & 7.867E-02 & 7.632E-06 & 8.645 & $1.133 \mathrm{E}-03$ \\
\hline 2800 & $49 E+01$ & $1.770 \mathrm{E}-01$ & $1.818 E+02$ & 4.208E-01 & $1.998 \mathrm{E}-05$ & 4.624 & 2.314E-03 \\
\hline 2800 & $30 E+02$ & $2.775 \mathrm{E}-01$ & $2.685 E+02$ & 6.596E-01 & 2.951E-05 & $7.24 \mathrm{~S}$ & $2.456 \mathrm{E}-03$ \\
\hline 2800 & $2.524 E+02$ & 7.073E-01 & $6.000 E+02$ & $1.681 E+00$ & $6.597 \mathrm{E}-$ & 1.84 & $2.801 \mathrm{E}-03$ \\
\hline 2800 & $4.059 E+02$ & $1.558 \mathrm{E}+00$ & $9.649 \mathrm{E}+02$ & $3.704 E+00$ & $1.061 E-04$ & 4.070 & $3.835 E-03$ \\
\hline 2800 & $4.698 E+02$ & $1.676 \mathrm{E}+00$ & $1.117 \mathrm{E}+03$ & $3.984 E+00$ & $1.228 \mathrm{E}-04$ & 4.378E-07 & \\
\hline & & & & & & & \\
\hline 2600 & $773 E+01$ & $3.453 \mathrm{E}-03$ & $4.214 E+01$ & 8.209E-03 & 4.631E-06 & 9.02 & \\
\hline 2600 & $407 E+01$ & 3.806E-03 & $3.343 E+01$ & 9.047E-03 & 3.674E-06 & 9.942 & $2.706 \mathrm{E}-04$ \\
\hline 2600 & $346 E+01$ & $9.468 \mathrm{E}-03$ & $5.577 \mathrm{E}+01$ & 2.251E-02 & $6.129 \mathrm{E}-06$ & 2.473 & 4.03 \\
\hline 2600 & $2.586 E+01$ & 1.136E-02 & $6.148 E+01$ & 2.700E-02 & 6.756E-06 & 2.968 & 4.39 \\
\hline 2600 & $4.475 E+01$ & 2.692E-02 & $1.064 E+02$ & 6.399E-02 & 1.169E-05 & 7.032 & $6.015 E-04$ \\
\hline 2600 & $855 E+01$ & 5.513E-02 & $1.629 E+02$ & 1.311E-01 & 1.791E-05 & $1.440 \mathrm{E}$ & 8.042E-04 \\
\hline 2600 & $8.640 E+01$ & 8.036E-02 & $2.054 E+02$ & $1.910 \mathrm{E}-01$ & 2.257E- & $2.099 \mathrm{E}$ & $9.299 \mathrm{E}-04$ \\
\hline 2600 & $1.063 E+02$ & 6.779E-02 & $2.526 E+02$ & 1.611E-01 & 2.777E- & & 6.376E-04 \\
\hline 2600 & $861 E+02$ & $2.124 \mathrm{E}-01$ & $4.425 E+02$ & $5.049 \mathrm{E}-01$ & 4.864E-05 & $5.548 \mathrm{E}-08$ & $1.141 \mathrm{E}-03$ \\
\hline 2600 & $2.851 E+02$ & 3.625E-01 & $6.777 \mathrm{E}+02$ & $8.618 \mathrm{E}-01$ & 7.452E-05 & $9.470 \mathrm{E}-08$ & $1.271 \mathrm{E}-03$ \\
\hline 2600 & $5.117 E+02$ & 6.737E-01 & $1.216 \mathrm{E}+03$ & $1.601 E+00$ & $1.338 \mathrm{E}-04$ & $1.760 \mathrm{E}-07$ & 1.315E-03 \\
\hline & & & & & & & \\
\hline 2000 & $183 E+01$ & $6.188 \mathrm{E}-03$ & $2.183 E+02$ & $1.471 \mathrm{E}-02$ & 2.399E-05 & 1.616E-09 & $6.738 E-05$ \\
\hline 2000 & $2.555 E+02$ & 2.503E-02 & $6.073 E+02$ & 5.949E-02 & $6.678 \mathrm{E}-05$ & 6.537E-09 & $9.790 \mathrm{E}-05$ \\
\hline 2000 & $4.367 E+02$ & 8.036E-02 & $1.038 E+03$ & $1.910 \mathrm{E}-01$ & $1.142 \mathrm{E}-04$ & 2.099E-08 & $1.838 E-04$ \\
\hline
\end{tabular}


Table C-2. Data of Goodstine [29] for partitioning of $\mathrm{Na}_{2} \mathrm{SO}_{4}$ between water and steam.

\begin{tabular}{|c|c|c|c|c|c|c|c|}
\hline \multirow[b]{2}{*}{$\begin{array}{c}\text { Pressure } \\
\text { (psig) }\end{array}$} & \multicolumn{2}{|c|}{ Concentration } & \multicolumn{2}{|c|}{ Concentration } & \multicolumn{2}{|c|}{ Concentration } & \multirow{2}{*}{$\begin{array}{c}\text { Partition } \\
\text { Coefficient } \\
(-)\end{array}$} \\
\hline & \multicolumn{2}{|c|}{ (ppm of $\mathrm{Na})$} & \multicolumn{2}{|c|}{ (ppm of $\mathrm{Na}_{2} \mathrm{SO}_{4}$ ) } & $\begin{array}{c}\text { Water } \\
\text { (mole fraction }\end{array}$ & $\begin{array}{c}\text { Steam } \\
\text { of } \mathrm{Na}_{2} \mathrm{SO}_{4} \text { ) }\end{array}$ & \\
\hline 3000 & $7.676 \mathrm{E}+00$ & $1.994 \mathrm{E}-02$ & $2.371 \mathrm{E}+01$ & $6.160 \mathrm{E}-02$ & $3.008 E-06$ & $7.813 \mathrm{E}-09$ & $2.598 \mathrm{E}-03$ \\
\hline 3000 & $8.159 E+00$ & 2.842E-02 & $2.521 E+01$ & 8.779E-02 & 3.197E-06 & 1.113E-08 & 3.483E-03 \\
\hline 3000 & $1.848 \mathrm{E}+01$ & $6.521 \mathrm{E}-02$ & $5.710 \mathrm{E}+01$ & 2.015E-01 & 7.242E-06 & 2.555E-08 & \\
\hline 3000 & 2.193E+01 & 7.551E-02 & $6.774 \mathrm{E}+01$ & 2.333E-01 & 8.592E-06 & 2.958E-08 & 3.443E-03 \\
\hline 3000 & $2.570 \mathrm{E}+01$ & 8.532E-02 & $7.939 E+01$ & 2.636E-01 & 1.007E-05 & 3.343E-08 & 3.320E-03 \\
\hline 3000 & $673 E+01$ & 1.819E-01 & $1.444 E+02$ & 5.621E-01 & $1.831 \mathrm{E}$ & 7.129E-08 & 3.893E-03 \\
\hline 3000 & $9.256 \mathrm{E}+01$ & 4.125E-01 & $2.859 E+02$ & $1.274 \mathrm{E}+00$ & $3.628 \mathrm{E}$ & 1.616E-07 & 4.455E-03 \\
\hline & $1.545 \mathrm{E}+02$ & 6.403E-01 & $4.774 \mathrm{E}+02$ & $1.978 \mathrm{E}+00$ & $6.058 \mathrm{E}$ & 2.509E-07 & 4.141E-03 \\
\hline 3000 & $2.880 E+02$ & $1.506 \mathrm{E}+00$ & 8.897E+02 & $4.651 E+00$ & $1.129 \mathrm{E}$ & 5.899 & $5.224 \mathrm{E}-03$ \\
\hline 2800 & $32 \mathrm{E}+00$ & 2.323E-03 & $1.585 E_{+}$ & 7.176E-03 & 6 & 9.10 & \\
\hline & $392 E+00$ & 3.077E-03 & $1.975 E+01$ & $9.504 \mathrm{E}-03$ & $2.505 E$ & 1.205E-09 & 4.813E-04 \\
\hline 2800 & $.539 E+01$ & $9.465 E-03$ & $4.755 \mathrm{E}+01$ & 2.924E-02 & $6.031 \mathrm{E}$ & 3.709E-09 & $6.149 E-04$ \\
\hline 2800 & $2.013 E+01$ & $1.853 \mathrm{E}-02$ & $6.219 E+01$ & 5.725E-02 & $7.888 \mathrm{E}$ & 7.261E-09 & $9.204 E-04$ \\
\hline 2800 & $2.275 E+01$ & 1.722E-02 & $7.027 E+01$ & 5.320E-02 & $8.912 E-$ & 6.748E-09 & 7.571E-04 \\
\hline & $302 E+01$ & 2.068E-02 & $7.113 E+01$ & $6.390 \mathrm{E}-02$ & $9.022 \mathrm{E}$ & 8.105E-09 & 8.983E-04 \\
\hline 2800 & $163 E+01$ & 5.046E-02 & $2.213 E+02$ & 1.559E-01 & 2.807E & 1.977E-08 & 7.042E-04 \\
\hline 2800 & $08 E+02$ & 5.701E-02 & $3.115 E+02$ & 1.761E-01 & $3.951 E$ & 2.234E-08 & 5.653E-04 \\
\hline 2800 & $44 \mathrm{E}+02$ & 2.690E-01 & $1.095 E+03$ & 8.309E-01 & $1.390 \mathrm{E}$ & & \\
\hline 2800 & $111 E+02$ & $3.928 \mathrm{E}-01$ & 1.579E+ & $1.213 \mathrm{E}+00$ & 2.005E- & $1.539 \mathrm{E}-07$ & 7.675E-04 \\
\hline 2600 & $08 E+00$ & & $547 E+01$ & $3.448 \mathrm{E}-03$ & $1.962 \mathrm{E}$ & & 2.229 \\
\hline & $E+00$ & $1.712 \mathrm{E}-03$ & $2.258 E+01$ & $5.288 \mathrm{E}-03$ & 2.86 & & 2.3 \\
\hline & $2 \mathrm{E}+01$ & $1.712 \mathrm{E}-03$ & $3.591 E+01$ & 5.288E-03 & $4.555 \mathrm{E}$ & $=-10$ & $1.472 \mathrm{E}-04$ \\
\hline 2600 & $346 E+01$ & 4.384E-03 & 4.157E+01 & 1.354E-02 & $5.273 \mathrm{E}$ & $E-09$ & 3.258E-04 \\
\hline 2600 & $963 E+00$ & 2.133E-03 & $2.460 \mathrm{E}+01$ & 6.588E-03 & $3.120 \mathrm{E}$ & $8.356 \mathrm{E}-10$ & 2.678E-04 \\
\hline 2600 & $389 E+01$ & $6.481 \mathrm{E}-03$ & $6.144 E+01$ & 2.002E-02 & 7.793E & 2.539E-09 & $3.259 E-04$ \\
\hline & $038 E+01$ & 7.147E-03 & $6.296 \mathrm{E}+01$ & 2.208E-02 & 7.985E & 2.800E-09 & 3.507E-04 \\
\hline 2600 & $965 E+01$ & 7.690E-03 & $6.069 \mathrm{E}+01$ & 2.376E-02 & $7.698 \mathrm{E}$ & 3.013E-09 & 3.914E-04 \\
\hline 2600 & $359 E+01$ & 7.597E-03 & $7.289 E+01$ & 2.347E-02 & $9.245 E-06$ & 2.977E-09 & $3.220 \mathrm{E}-04$ \\
\hline 2600 & $570 E+01$ & 7.505E-03 & $7.939 E+01$ & 2.318E-02 & 1.007E-05 & 2.940E-09 & $2.920 \mathrm{E}-04$ \\
\hline 2600 & $903 E+01$ & $8.175 E-03$ & $8.969 E+01$ & 2.525E-02 & $1.138 \mathrm{E}$ & 3.203E-09 & 2.815E-0 \\
\hline 2600 & $601 E+01$ & 8.796E-03 & $8.036 E+01$ & 2.717E-02 & $1.019 \mathrm{E}-05$ & 3.446E-09 & 3.381E-04 \\
\hline 2600 & $.508 \mathrm{E}+01$ & $9.939 \mathrm{E}-03$ & 7.747E+01 & 3.070E-02 & 9.827E-06 & 3.894E-09 & 3.963E-04 \\
\hline 2600 & $395 E+01$ & 1.994E-02 & $2.593 E+02$ & $6.160 \mathrm{E}-02$ & $3.290 \mathrm{E}-05$ & 7.813E-09 & 2.375E-04 \\
\hline 2600 & $9.485 E+01$ & 1.994E-02 & $2.930 \mathrm{E}+02$ & 6.160E-02 & 3.717E-05 & 7.813E-09 & 2.102E-04 \\
\hline 2600 & $2.518 \mathrm{E}+02$ & 5.363E-02 & $7.779 E+02$ & 1.657E-01 & 9.873E-05 & 2.101E-08 & 2.129E-04 \\
\hline 2600 & $2.398 \mathrm{E}+02$ & $5.701 \mathrm{E}-02$ & $7.408 \mathrm{E}+02$ & 1.761E-01 & 9.402E-05 & 2.234E-08 & 2.376E-04 \\
\hline 2600 & $4.579 E+02$ & $1.201 \mathrm{E}-01$ & $1.415 E+03$ & $3.710 \mathrm{E}-01$ & 1.796E-04 & 4.706E-08 & 2.620E-04 \\
\hline 2600 & $4.750 \mathrm{E}+02$ & 1.292E-01 & $1.467 E+03$ & 3.993E-01 & 1.863E-04 & 5.064E-08 & $2.718 \mathrm{E}-04$ \\
\hline
\end{tabular}




\section{APPENDIX D - COMPARISON OF EXTRAPOLATION PROPERTIES}

\begin{tabular}{|c|c|c|c|c|c|c|}
\hline \multirow[t]{2}{*}{$\begin{array}{c}\text { Temperature } \\
\left({ }^{\circ} \mathrm{C}\right)\end{array}$} & \multirow[t]{2}{*}{$\begin{array}{l}\text { Pressure } \\
\text { (MPa) }\end{array}$} & \multirow[t]{2}{*}{$\begin{array}{l}\text { Density } \\
(\mathrm{mol} / \mathrm{L})\end{array}$} & \multicolumn{2}{|c|}{$\begin{array}{l}\text { Predicted solubility } \\
\text { (mol fraction) }\end{array}$} & \multicolumn{2}{|c|}{$\begin{array}{c}\text { Pred. sol./reference sol. } \\
(-)\end{array}$} \\
\hline & & & $\mathrm{SiO}_{2}$ & $\mathrm{NaCl}$ & $\mathrm{SiO}_{2}$ & $\mathrm{NaCl}$ \\
\hline 350 & 16.52942 & 6.306074 & $1.149 \mathrm{E}-04$ & 3.589E-05 & $1.000 E+00$ & $1.000 \mathrm{E}+00$ \\
\hline 300 & 2.24500 & 0.5 & 3.182E-07 & 1.493E-09 & $2.770 \mathrm{E}-03$ & 4.158E-05 \\
\hline 300 & 4.21000 & 1.0 & 9.821E-07 & 1.800 E-08 & 8.548E-03 & 5.016E-04 \\
\hline 300 & 5.89600 & 1.5 & 1.973E-06 & 7.970E-08 & 1.717E-02 & 2.220E-03 \\
\hline 300 & 7.30900 & 2.0 & 3.343E-06 & $2.346 \mathrm{E}-07$ & 2.909E-02 & $6.536 \mathrm{E}-03$ \\
\hline 300 & 8.46100 & 2.5 & 5.177E-06 & 5.531E-07 & 4.505E-02 & 1.541E-02 \\
\hline 350 & 2.47400 & 0.5 & 6.073E-07 & 2.672E-09 & 5.286E-03 & 7.443E-05 \\
\hline 350 & 4.71300 & 1.0 & 1.935E-06 & $3.172 E-08$ & 1.684E-02 & 8.838E-04 \\
\hline 350 & 6.72100 & 1.5 & 3.958E-06 & $1.379 \mathrm{E}-07$ & 3.445E-02 & 3.842E-03 \\
\hline 350 & 8.50600 & 2.0 & 6.796E-06 & 3.976E-07 & 5.915E-02 & $1.108 E-02$ \\
\hline 350 & 10.08000 & 2.5 & 1.063E-05 & $9.158 E-07$ & $9.254 \mathrm{E}-02$ & $2.551 E-02$ \\
\hline 350 & 11.45000 & 3.0 & $1.572 E-05$ & $1.831 \mathrm{E}-06$ & $1.368 \mathrm{E}-01$ & 5.102E-02 \\
\hline 350 & 12.63000 & 3.5 & 2.237E-05 & 3.322E-06 & 1.947E-01 & $9.254 \mathrm{E}-02$ \\
\hline 350 & 13.65000 & 4.0 & 3.099E-05 & $5.605 E-06$ & 2.697E-01 & $1.562 E-01$ \\
\hline 350 & 14.51000 & 4.5 & 4.210E-05 & 8.958E-06 & 3.664E-01 & 2.496E-01 \\
\hline 350 & 15.22000 & 5.0 & 5.635E-05 & $1.372 E-05$ & 4.904E-01 & 3.822E-01 \\
\hline 400 & 2.69700 & 0.5 & 1.159E-06 & 4.370E-09 & $1.009 \mathrm{E}-02$ & 1.217E-04 \\
\hline 400 & 5.19400 & 1.0 & 3.811E-06 & $5.133 \mathrm{E}-08$ & 3.317E-02 & 1.430 E-03 \\
\hline 400 & 7.49300 & 1.5 & 7.942E-06 & 2.206E-07 & 6.913E-02 & $6.145 E-03$ \\
\hline 400 & 9.60200 & 2.0 & $1.382 E-05$ & $6.281 E-07$ & 1.203E-01 & 1.750 E-02 \\
\hline 400 & 11.53000 & 2.5 & 2.184E-05 & $1.428 \mathrm{E}-06$ & 1.901E-01 & 3.977E-02 \\
\hline 400 & 13.28000 & 3.0 & 3.255E-05 & $2.815 \mathrm{E}-06$ & 2.833E-01 & 7.844E-02 \\
\hline 400 & 14.87000 & 3.5 & 4.665E-05 & 5.031E-06 & $4.060 \mathrm{E}-01$ & $1.402 E-01$ \\
\hline 400 & 16.31000 & 4.0 & 6.503E-05 & 8.365E-06 & 5.660 E- 01 & 2.330E-01 \\
\hline 400 & 17.61000 & 4.5 & 8.882E-05 & $1.316 \mathrm{E}-05$ & 7.731E-01 & 3.667E-01 \\
\hline 400 & 18.79000 & 5.0 & 1.195E-04 & 1.982E-05 & $1.040 E+00$ & 5.521E-01 \\
\hline 450 & 2.91800 & 0.5 & $2.212 \mathrm{E}-06$ & 6.649E-09 & $1.925 \mathrm{E}-02$ & $1.852 E-04$ \\
\hline 450 & 5.66100 & 1.0 & 7.506E-06 & $7.753 E-08$ & $6.533 \mathrm{E}-02$ & $2.160 \mathrm{E}-03$ \\
\hline 450 & 8.23300 & 1.5 & $1.594 \mathrm{E}-05$ & 3.305E-07 & 1.387E-01 & $9.206 \mathrm{E}-03$ \\
\hline 450 & 10.64000 & 2.0 & 2.809E-05 & 9.331E-07 & 2.445E-01 & 2.599E-02 \\
\hline 450 & 12.89000 & 2.5 & 4.485E-05 & 2.102E-06 & 3.904E-01 & 5.856E-02 \\
\hline 450 & 14.98000 & 3.0 & $6.741 \mathrm{E}-05$ & 4.109E-06 & 5.867E-01 & $1.145 E-01$ \\
\hline 450 & 16.94000 & 3.5 & $9.729 E-05$ & 7.270 E-06 & 8.468E-01 & $2.025 E-01$ \\
\hline 450 & 18.76000 & 4.0 & 1.365E-04 & 1.197E-05 & $1.188 E+00$ & 3.335E-01 \\
\hline 450 & 20.45000 & 4.5 & 1.874E-04 & $1.866 \mathrm{E}-05$ & $1.631 E+00$ & 5.198E-01 \\
\hline 450 & 22.03000 & 5.0 & 2.532E-04 & 2.782E-05 & $2.204 E+00$ & 7.752E-01 \\
\hline 500 & 3.13600 & 0.5 & 4.222E-06 & $9.548 \mathrm{E}-09$ & $3.674 \mathrm{E}-02$ & $2.660 \mathrm{E}-04$ \\
\hline 500 & 6.11900 & 1.0 & 1.479E-05 & 1.107E-07 & 1.287E-01 & $3.084 E-03$ \\
\hline 500 & 8.95200 & 1.5 & $3.198 \mathrm{E}-05$ & 4.691E-07 & 2.783E-01 & 1.307E-02 \\
\hline 500 & 11.64000 & 2.0 & 5.711E-05 & $1.316 \mathrm{E}-06$ & 4.971E-01 & 3.667E-02 \\
\hline 500 & 14.19000 & 2.5 & $9.213 E-05$ & 2.947E-06 & 8.018E-01 & $8.210 E-02$ \\
\hline 500 & 16.60000 & 3.0 & 1.396E-04 & 5.722E-06 & $1.215 E+00$ & $1.594 \mathrm{E}-01$ \\
\hline 500 & 18.89000 & 3.5 & 2.029E-04 & $1.006 \mathrm{E}-05$ & $1.766 \mathrm{E}+00$ & 2.803E-01 \\
\hline 500 & 21.06000 & 4.0 & 2.864E-04 & 1.646E-05 & $2.492 \mathrm{E}+00$ & 4.585E-01 \\
\hline
\end{tabular}

${ }^{*}$ Reference Point 


\begin{tabular}{|c|c|c|c|c|c|c|}
\hline \multirow[t]{2}{*}{$\begin{array}{c}\text { Temperature } \\
\left({ }^{\circ} \mathrm{C}\right)\end{array}$} & \multirow[t]{2}{*}{$\begin{array}{l}\text { Pressure } \\
\text { (MPa) }\end{array}$} & \multirow[t]{2}{*}{$\begin{array}{l}\text { Density } \\
(\mathrm{mol} / \mathrm{L})\end{array}$} & \multicolumn{2}{|c|}{$\begin{array}{l}\text { Predicted solubility } \\
\quad \text { (mol fraction) }\end{array}$} & \multicolumn{2}{|c|}{$\begin{array}{c}\text { Pred. sol./reference sol. } \\
(-)\end{array}$} \\
\hline & & & $\mathrm{SiO}_{2}$ & $\mathrm{NaCl}$ & $\mathrm{SiO}_{2}$ & $\mathrm{NaCl}$ \\
\hline 500 & 23.12000 & 4.5 & $3.954 \mathrm{E}-04$ & 2.547E-05 & $3.441 E+00$ & $7.096 \mathrm{E}-01$ \\
\hline 500 & 25.07000 & 5.0 & 5.369E-04 & 3.773E-05 & $4.672 E+00$ & $1.051 E+00$ \\
\hline 550 & 3.35300 & 0.5 & 8.057E-06 & 1.307E-08 & $7.012 E-02$ & 3.642E-04 \\
\hline 550 & 6.57200 & 1.0 & 2.913E-05 & 1.509E-07 & $2.535 E-01$ & 4.204E-03 \\
\hline 550 & 9.65700 & 1.5 & 6.417E-05 & 6.366E-07 & 5.585E-01 & 1.774E-02 \\
\hline 550 & 12.62000 & 2.0 & 1.161E-04 & 1.778E-06 & $1.011 E+00$ & 4.952E-02 \\
\hline 550 & 15.45000 & 2.5 & $1.892 \mathrm{E}-04$ & 3.963E-06 & 1.647E+00 & 1.104E-01 \\
\hline 550 & 18.17000 & 3.0 & 2.892E-04 & 7.654E-06 & $2.517 E+00$ & 2.132E-01 \\
\hline 550 & 20.77000 & 3.5 & 4.233E-04 & $1.340 E-05$ & $3.684 \mathrm{E}+00$ & 3.732E-01 \\
\hline 550 & 23.27000 & 4.0 & $6.009 E-04$ & 2.181E-05 & $5.230 \mathrm{E}+00$ & $6.075 E-01$ \\
\hline 550 & 25.67000 & 4.5 & 8.341E-04 & 3.359E-05 & $7.260 \mathrm{E}+00$ & 9.357E-01 \\
\hline 550 & 27.98000 & 5.0 & $1.138 \mathrm{E}-03$ & 4.950E-05 & $9.905 \mathrm{E}+00$ & $1.379 E+00$ \\
\hline 600 & 3.56900 & 0.5 & $1.538 \mathrm{E}-05$ & $1.722 \mathrm{E}-08$ & $1.338 \mathrm{E}-01$ & 4.796E-04 \\
\hline 600 & 7.01900 & 1.0 & 5.737E-05 & $1.980 \mathrm{E}-07$ & 4.993E-01 & 5.517E-03 \\
\hline 600 & 10.35000 & 1.5 & $1.288 \mathrm{E}-04$ & 8.325E-07 & $1.121 \mathrm{E}+00$ & 2.319E-02 \\
\hline 600 & 13.57000 & 2.0 & 2.361E-04 & 2.317E-06 & $2.055 E+00$ & $6.455 \mathrm{E}-02$ \\
\hline 600 & 16.68000 & 2.5 & 3.886E-04 & 5.145E-06 & $3.383 E+00$ & $1.433 \mathrm{E}-01$ \\
\hline 600 & 19.69000 & 3.0 & 5.989E-04 & 9.899E-06 & $5.212 \mathrm{E}+00$ & 2.758E-01 \\
\hline 600 & 22.60000 & 3.5 & 8.828E-04 & 1.726E-05 & $7.683 E+00$ & 4.808E-01 \\
\hline 600 & 25.41000 & 4.0 & $1.261 \mathrm{E}-03$ & 2.799E-05 & $1.098 E+01$ & 7.798E-01 \\
\hline 600 & 28.14000 & 4.5 & $1.760 \mathrm{E}-03$ & 4.294E-05 & $1.532 E+01$ & 1.196E+00 \\
\hline 600 & 30.78000 & 5.0 & 2.413E-03 & 6.307E-05 & $2.100 \mathrm{E}+01$ & 1.757E+00 \\
\hline 650 & 3.78400 & 0.5 & 2.934E-05 & 2.194E-08 & $2.554 \mathrm{E}-01$ & 6.112E-04 \\
\hline 650 & 7.46300 & 1.0 & $1.130 \mathrm{E}-04$ & 2.516E-07 & $9.836 \mathrm{E}-01$ & 7.011E-03 \\
\hline 650 & 11.04000 & 1.5 & 2.584E-04 & 1.055E-06 & $2.249 E+00$ & $2.938 \mathrm{E}-02$ \\
\hline 650 & 14.51000 & 2.0 & 4.800E-04 & 2.928E-06 & 4.177E+00 & 8.157E-02 \\
\hline 650 & 17.90000 & 2.5 & 7.983E-04 & 6.478E-06 & $6.948 E+00$ & $1.805 E-01$ \\
\hline 650 & 21.18000 & 3.0 & $1.240 \mathrm{E}-03$ & 1.243E-05 & $1.079 \mathrm{E}+01$ & 3.464E-01 \\
\hline 650 & 24.39000 & 3.5 & $1.841 \mathrm{E}-03$ & 2.161E-05 & $1.602 E+01$ & $6.019 \mathrm{E}-01$ \\
\hline 650 & 27.51000 & 4.0 & 2.646E-03 & 3.493E-05 & $2.303 E+01$ & $9.732 E-01$ \\
\hline 650 & 30.55000 & 4.5 & 3.713E-03 & 5.344E-05 & $3.231 E+01$ & $1.489 \mathrm{E}+00$ \\
\hline 650 & 33.52000 & 5.0 & 5.115E-03 & 7.825E-05 & $4.452 E+01$ & $2.180 E+00$ \\
\hline 700 & 3.99900 & 0.5 & $5.600 \mathrm{E}-05$ & 2.720E-08 & 4.874E-01 & 7.577E-04 \\
\hline 700 & 7.90400 & 1.0 & $2.226 \mathrm{E}-04$ & 3.113E-07 & 1.937E+00 & 8.672E-03 \\
\hline 700 & 11.72000 & 1.5 & 5.185E-04 & 1.301E-06 & $4.512 \mathrm{E}+00$ & 3.626E-02 \\
\hline 700 & 15.45000 & 2.0 & $9.758 \mathrm{E}-04$ & 3.602E-06 & $8.493 E+00$ & $1.004 \mathrm{E}-01$ \\
\hline 700 & 19.09000 & 2.5 & $1.640 \mathrm{E}-03$ & 7.957E-06 & 1.427E+01 & 2.217E-01 \\
\hline 700 & 22.65000 & 3.0 & $2.569 \mathrm{E}-03$ & 1.523E-05 & $2.236 \mathrm{E}+01$ & 4.244E-01 \\
\hline 700 & 26.14000 & 3.5 & $3.840 \mathrm{E}-03$ & 2.641E-05 & $3.342 \mathrm{E}+01$ & 7.358E-01 \\
\hline 700 & 29.56000 & 4.0 & 5.553E-03 & 4.259E-05 & $4.833 E+01$ & 1.187E+00 \\
\hline 700 & 32.91000 & 4.5 & 7.833E-03 & $6.499 \mathrm{E}-05$ & $6.818 \mathrm{E}+01$ & $1.811 \mathrm{E}+00$ \\
\hline 700 & 36.19000 & 5.0 & 1.084E-02 & $9.495 \mathrm{E}-05$ & $9.437 \mathrm{E}+01$ & $2.645 E+00$ \\
\hline
\end{tabular}




\section{APPENDIX E - SAMPLE SOLUBILITY CALCULATIONS}

Table E-1. $\mathrm{NaCl}$ solubility equation.

$$
\begin{array}{rlll}
\mathbf{X} & =\boldsymbol{\rho}^{\mathrm{M}} \exp (\mathrm{A}+\mathrm{B} / \mathrm{T}) / \mathbf{P} \\
\mathrm{M}=\mathrm{m}_{1}+\mathrm{m}_{3} / \mathbf{T} & & & \\
\mathrm{A}= & -7.92886 \mathrm{E}+00 & \mathrm{~m}_{1}= & 4.49961 \mathrm{E}+00 \\
\mathrm{~B}= & -4.85247 \mathrm{E}+03 & \mathrm{~m}_{3}= & -1.30000 \mathrm{E}-03
\end{array}
$$

Solubility (mole fraction)

\begin{tabular}{c|cccccc} 
Temperature & \multicolumn{6}{|c}{ Pressure $(\mathrm{MPa})$} \\
\cline { 2 - 7 }$\left({ }^{\circ} \mathrm{C}\right)$ & 1.0 & 2.0 & 3.0 & 4.0 & 5.0 & 6.0 \\
\hline 200 & $3.4657 \mathrm{E}-11$ & - & - & - & - & - \\
300 & $7.5395 \mathrm{E}-11$ & $9.6468 \mathrm{E}-10$ & $4.5680 \mathrm{E}-09$ & $1.4545 \mathrm{E}-08$ & $3.7656 \mathrm{E}-08$ & $8.6585 \mathrm{E}-08$ \\
400 & $1.2195 \mathrm{E}-10$ & $1.4671 \mathrm{E}-09$ & $6.4661 \mathrm{E}-09$ & $1.8924 \mathrm{E}-08$ & $4.4320 \mathrm{E}-08$ & $9.0265 \mathrm{E}-08$ \\
500 & $1.6203 \mathrm{E}-10$ & $1.8980 \mathrm{E}-09$ & $8.1298 \mathrm{E}-09$ & $2.3077 \mathrm{E}-08$ & $5.2302 \mathrm{E}-08$ & $1.0284 \mathrm{E}-07$ \\
600 & $1.8978 \mathrm{E}-10$ & $2.1924 \mathrm{E}-09$ & $9.2564 \mathrm{E}-09$ & $2.5886 \mathrm{E}-08$ & $5.7770 \mathrm{E}-08$ & $1.1179 \mathrm{E}-07$ \\
700 & $2.0463 \mathrm{E}-10$ & $2.3450 \mathrm{E}-09$ & $9.8203 \mathrm{E}-09$ & $2.7235 \mathrm{E}-08$ & $6.0266 \mathrm{E}-08$ & $1.1561 \mathrm{E}-07$ \\
800 & $2.0868 \mathrm{E}-10$ & $2.3797 \mathrm{E}-09$ & $9.9160 \mathrm{E}-09$ & $2.7362 \mathrm{E}-08$ & $6.0238 \mathrm{E}-08$ & $1.1496 \mathrm{E}-07$ \\
900 & $2.0483 \mathrm{E}-10$ & $2.3283 \mathrm{E}-09$ & $9.6708 \mathrm{E}-09$ & $2.6599 \mathrm{E}-08$ & $5.8369 \mathrm{E}-08$ & $1.1103 \mathrm{E}-07$
\end{tabular}

Table E-2. $\mathrm{SiO}_{2}$ solubility equation.

$$
\begin{aligned}
& \mathbf{x}=\rho^{\mathrm{M}} \exp (\mathrm{A}+\mathrm{DT}) / \mathbf{P} \\
& M=m_{1}+m_{2} \rho+m_{3} T \\
& A=\quad-2.16047 E+01 \\
& D=\quad 1.35587 \mathrm{E}-02
\end{aligned}
$$$$
\mathrm{m}_{1}=\quad 1.05555 \mathrm{E}+00
$$$$
\mathrm{m}_{2}=\quad 9.41005 \mathrm{E}-02
$$$$
m_{3}=\quad 9.12888 E-04
$$

Solubility (mole fraction)

\begin{tabular}{c|cccccc} 
Temperature & \multicolumn{6}{|c}{ Pressure $(\mathrm{MPa})$} \\
\cline { 2 - 7 }$\left({ }^{\circ} \mathrm{C}\right)$ & 1.0 & 2.0 & 3.0 & 4.0 & 5.0 & 6.0 \\
\hline 200 & $3.4808 \mathrm{E}-08$ & - & - & - & - & - \\
300 & $8.4185 \mathrm{E}-08$ & $1.3092 \mathrm{E}-07$ & $1.7530 \mathrm{E}-07$ & $2.2260 \mathrm{E}-07$ & $2.7667 \mathrm{E}-07$ & $3.4208 \mathrm{E}-07$ \\
400 & $2.1321 \mathrm{E}-07$ & $3.4521 \mathrm{E}-07$ & $4.6578 \mathrm{E}-07$ & $5.8527 \mathrm{E}-07$ & $7.0896 \mathrm{E}-07$ & $8.4069 \mathrm{E}-07$ \\
500 & $5.5015 \mathrm{E}-07$ & $9.3890 \mathrm{E}-07$ & $1.2978 \mathrm{E}-06$ & $1.6495 \mathrm{E}-06$ & $2.0049 \mathrm{E}-06$ & $2.3715 \mathrm{E}-06$ \\
600 & $1.4331 \mathrm{E}-06$ & $2.5904 \mathrm{E}-06$ & $3.6896 \mathrm{E}-06$ & $4.7756 \mathrm{E}-06$ & $5.8714 \mathrm{E}-06$ & $6.9926 \mathrm{E}-06$ \\
700 & $3.7524 \mathrm{E}-06$ & $7.2003 \mathrm{E}-06$ & $1.0596 \mathrm{E}-05$ & $1.4010 \mathrm{E}-05$ & $1.7484 \mathrm{E}-05$ & $2.1046 \mathrm{E}-05$ \\
800 & $9.8515 \mathrm{E}-06$ & $2.0094 \mathrm{E}-05$ & $3.0598 \mathrm{E}-05$ & $4.1396 \mathrm{E}-05$ & $5.2529 \mathrm{E}-05$ & $6.4039 \mathrm{E}-05$ \\
900 & $2.5894 \mathrm{E}-05$ & $5.6182 \mathrm{E}-05$ & $8.8603 \mathrm{E}-05$ & $1.2278 \mathrm{E}-04$ & $1.5859 \mathrm{E}-04$ & $1.9603 \mathrm{E}-04$
\end{tabular}


Table $\mathrm{E}-3 . \quad \mathrm{Na}_{3} \mathrm{PO}_{4}$ solubility equation.

$$
\begin{aligned}
& X=x_{\text {ref }}{ }^{*} X_{\mathrm{NaCl}}(T, P, \rho) / x_{\mathrm{NaCl}}\left(T_{\text {ref }}, P_{\text {ref }}, \rho_{\text {ref }}\right) \\
& x_{\mathrm{NaCl}}=\rho^{\mathrm{M}} \exp (\mathrm{A}+\mathrm{B} / \mathrm{T}) / \mathbf{P} \\
& M=m_{1}+m_{3} / T \\
& \mathrm{~A}=\quad-7.92886 \mathrm{E}+00 \quad \mathrm{~m}_{1}=\quad 4.49961 \mathrm{E}+00 \\
& B=-4.85247 E+03 \quad m_{3}=-1.30000 E-03 \\
& \mathrm{~T}_{\text {ret }}=623.15 \mathrm{~K} \quad \mathrm{P}_{\text {ref }}=16.52942 \mathrm{MPa} \\
& \rho_{\text {ref }}=6.30607388 \mathrm{~mol} / \mathrm{dm}^{3} \quad \mathrm{x}_{\text {ref }}=2.8000 \mathrm{E}-07 \mathrm{~mole} \mathrm{fr} . \\
& \mathrm{x}_{\mathrm{NaCl}}\left(\mathrm{T}_{\mathrm{ret}}, \mathrm{P}_{\text {ret }}, \rho_{\mathrm{ret}}\right)=3.8518 \mathrm{E}-04 \text { mole fr. }
\end{aligned}
$$

Solubility (mole fraction)

\begin{tabular}{c|cccccc}
\multirow{2}{*}{$\begin{array}{c}\text { Temperature } \\
\left({ }^{\circ} \mathrm{C}\right)\end{array}$} & \multicolumn{7}{|c}{ Pressure $(\mathrm{MPa})$} \\
\cline { 2 - 7 } & 1.0 & 2.0 & 3.0 & 4.0 & 5.0 & 6.0 \\
\hline 200 & $2.5193 \mathrm{E}-14$ & - & - & - & - & - \\
300 & $5.4807 \mathrm{E}-14$ & $7.0126 \mathrm{E}-13$ & $3.3207 \mathrm{E}-12$ & $1.0573 \mathrm{E}-11$ & $2.7374 \mathrm{E}-11$ & $6.2942 \mathrm{E}-11$ \\
400 & $8.8649 \mathrm{E}-14$ & $1.0665 \mathrm{E}-12$ & $4.7005 \mathrm{E}-12$ & $1.3757 \mathrm{E}-11$ & $3.2218 \mathrm{E}-11$ & $6.5617 \mathrm{E}-11$ \\
500 & $1.1779 \mathrm{E}-13$ & $1.3797 \mathrm{E}-12$ & $5.9098 \mathrm{E}-12$ & $1.6775 \mathrm{E}-11$ & $3.8020 \mathrm{E}-11$ & $7.4757 \mathrm{E}-11$ \\
600 & $1.3796 \mathrm{E}-13$ & $1.5937 \mathrm{E}-12$ & $6.7288 \mathrm{E}-12$ & $1.8818 \mathrm{E}-11$ & $4.1995 \mathrm{E}-11$ & $8.1263 \mathrm{E}-11$ \\
700 & $1.4875 \mathrm{E}-13$ & $1.7047 \mathrm{E}-12$ & $7.1387 \mathrm{E}-12$ & $1.9798 \mathrm{E}-11$ & $4.3809 \mathrm{E}-11$ & $8.4043 \mathrm{E}-11$ \\
800 & $1.5170 \mathrm{E}-13$ & $1.7299 \mathrm{E}-12$ & $7.2083 \mathrm{E}-12$ & $1.9890 \mathrm{E}-11$ & $4.3789 \mathrm{E}-11$ & $8.3571 \mathrm{E}-11$ \\
900 & $1.4890 \mathrm{E}-13$ & $1.6926 \mathrm{E}-12$ & $7.0301 \mathrm{E}-12$ & $1.9336 \mathrm{E}-11$ & $4.2430 \mathrm{E}-11$ & $8.0714 \mathrm{E}-11$
\end{tabular}

Table E-4. $\mathrm{Na}_{2} \mathrm{SO}_{4}$ solubility equation.

$$
\begin{aligned}
& \mathbf{x}=\mathrm{X}_{\mathrm{ref}}{ }^{*} \mathrm{X}_{\mathrm{NaCl}}(T, P, \rho) / \mathrm{X}_{\mathrm{NaCl}}\left(T_{\mathrm{ref}}, \mathrm{P}_{\mathrm{ref}}, \rho_{\mathrm{ref}}\right) \\
& x_{\mathrm{NaCl}}=\rho^{M} \exp (A+B / T) / P \\
& M=m_{1}+m_{3} / T \\
& \mathrm{~A}=-7.92886 \mathrm{E}+00 \quad \mathrm{~m}_{1}=\quad 4.49961 \mathrm{E}+00 \\
& \mathrm{~B}=\quad-4.85247 \mathrm{E}+03 \quad \mathrm{~m}_{3}=\quad-1.30000 \mathrm{E}-03 \\
& \mathrm{~T}_{\text {ref }}=633.15 \mathrm{~K} \quad \mathrm{P}_{\text {rel }}=18.66601 \mathrm{MPa} \\
& \rho_{\text {ref }}=7.987581 \mathrm{~mol} / \mathrm{dm}^{3} \quad \mathrm{X}_{\text {ref }}=1.5000 \mathrm{E}-06 \mathrm{~mole} \mathrm{fr} . \\
& x_{\text {Nacl }}\left(T_{\text {ret }}, P_{\text {ret }}, \rho_{\text {ret }}\right)=1.0489 E-03 \text { mole fr. }
\end{aligned}
$$

Solubility (mole fraction)

\begin{tabular}{c|cccccc} 
Temperature & \multicolumn{6}{|c}{ Pressure $(\mathrm{MPa})$} \\
\cline { 2 - 7 }$\left({ }^{\circ} \mathrm{C}\right)$ & 1.0 & 2.0 & 3.0 & 4.0 & 5.0 & 6.0 \\
\hline 200 & $4.9562 \mathrm{E}-14$ & - & - & - & - & - \\
300 & $1.0782 \mathrm{E}-13$ & $1.3796 \mathrm{E}-12$ & $6.5327 \mathrm{E}-12$ & $2.0801 \mathrm{E}-11$ & $5.3852 \mathrm{E}-11$ & $1.2383 \mathrm{E}-10$ \\
400 & $1.7440 \mathrm{E}-13$ & $2.0981 \mathrm{E}-12$ & $9.2473 \mathrm{E}-12$ & $2.7064 \mathrm{E}-11$ & $6.3382 \mathrm{E}-11$ & $1.2909 \mathrm{E}-10$ \\
500 & $2.3172 \mathrm{E}-13$ & $2.7143 \mathrm{E}-12$ & $1.1626 \mathrm{E}-11$ & $3.3002 \mathrm{E}-11$ & $7.4797 \mathrm{E}-11$ & $1.4707 \mathrm{E}-10$ \\
600 & $2.7140 \mathrm{E}-13$ & $3.1353 \mathrm{E}-12$ & $1.3238 \mathrm{E}-11$ & $3.7020 \mathrm{E}-11$ & $8.2617 \mathrm{E}-11$ & $1.5987 \mathrm{E}-10$ \\
700 & $2.9264 \mathrm{E}-13$ & $3.3536 \mathrm{E}-12$ & $1.4044 \mathrm{E}-11$ & $3.8949 \mathrm{E}-11$ & $8.6186 \mathrm{E}-11$ & $1.6534 \mathrm{E}-10$ \\
800 & $2.9844 \mathrm{E}-13$ & $3.4032 \mathrm{E}-12$ & $1.4181 \mathrm{E}-11$ & $3.9130 \mathrm{E}-11$ & $8.6146 \mathrm{E}-11$ & $1.6441 \mathrm{E}-10$ \\
900 & $2.9293 \mathrm{E}-13$ & $3.3298 \mathrm{E}-12$ & $1.3830 \mathrm{E}-11$ & $3.8039 \mathrm{E}-11$ & $8.3473 \mathrm{E}-11$ & $1.5879 \mathrm{E}-10$
\end{tabular}


Table E-5. CuO solubility equation based on ref. 38 .

$$
\begin{aligned}
\mathbf{x} & =\rho^{M} \exp (A+B / T) / P \\
M & =m_{1}+m_{3} / \mathbf{T}
\end{aligned}
$$

$\begin{array}{llll}A= & -9.75084 \mathrm{E}+00 & \mathrm{~m}_{1}= & 1.14584 \mathrm{E}+00 \\ \mathrm{~B}= & -4.85247 \mathrm{E}+03 & \mathrm{~m}_{3}= & -1.30000 \mathrm{E}-03\end{array}$

Solubility (mole fraction)

\begin{tabular}{c|cccccc} 
Temperature & \multicolumn{6}{|c}{ Pressure $(\mathrm{MPa})$} \\
\cline { 2 - 7 }$\left({ }^{\circ} \mathrm{C}\right)$ & 1.0 & 2.0 & 3.0 & 4.0 & 5.0 & 6.0 \\
\hline 200 & $4.5568 \mathrm{E}-10$ & - & - & - & - & - \\
300 & $2.1078 \mathrm{E}-09$ & $2.4065 \mathrm{E}-09$ & $2.6430 \mathrm{E}-09$ & $2.8646 \mathrm{E}-09$ & $3.0906 \mathrm{E}-09$ & $3.3351 \mathrm{E}-09$ \\
400 & $6.0833 \mathrm{E}-09$ & $6.8370 \mathrm{E}-09$ & $7.3733 \mathrm{E}-09$ & $7.8217 \mathrm{E}-09$ & $8.2259 \mathrm{E}-09$ & $8.6066 \mathrm{E}-09$ \\
500 & $1.3103 \mathrm{E}-08$ & $1.4627 \mathrm{E}-08$ & $1.5660 \mathrm{E}-08$ & $1.6483 \mathrm{E}-08$ & $1.7191 \mathrm{E}-08$ & $1.7826 \mathrm{E}-08$ \\
600 & $2.3310 \mathrm{E}-08$ & $2.5928 \mathrm{E}-08$ & $2.7658 \mathrm{E}-08$ & $2.9002 \mathrm{E}-08$ & $3.0129 \mathrm{E}-08$ & $3.1115 \mathrm{E}-08$ \\
700 & $3.6369 \mathrm{E}-08$ & $4.0372 \mathrm{E}-08$ & $4.2975 \mathrm{E}-08$ & $4.4968 \mathrm{E}-08$ & $4.6614 \mathrm{E}-08$ & $4.8034 \mathrm{E}-08$ \\
800 & $5.1678 \mathrm{E}-08$ & $5.7294 \mathrm{E}-08$ & $6.0911 \mathrm{E}-08$ & $6.3654 \mathrm{E}-08$ & $6.5898 \mathrm{E}-08$ & $6.7816 \mathrm{E}-08$ \\
900 & $6.8550 \mathrm{E}-08$ & $7.5938 \mathrm{E}-08$ & $8.0666 \mathrm{E}-08$ & $8.4230 \mathrm{E}-08$ & $8.7126 \mathrm{E}-08$ & $8.9588 \mathrm{E}-08$
\end{tabular}

Table E-6. CuO solubility equation based on ref. 37 .

$$
\begin{aligned}
\mathbf{x} & =\rho^{M} \exp (A+B / T) / P \\
M & =m_{1}+m_{3} / T
\end{aligned}
$$

$\begin{array}{llll}A= & -1.49399 \mathrm{E}+01 & \mathrm{~m}_{1}= & 2.59618 \mathrm{E}+00 \\ \mathrm{~B}= & -4.85247 \mathrm{E}+03 & \mathrm{~m}_{3}= & -1.30000 \mathrm{E}-03\end{array}$

Solubility (mole fraction)

\begin{tabular}{c|cccccc} 
Temperature & \multicolumn{7}{|c}{ Pressure $(\mathrm{MPa})$} \\
\cline { 2 - 7 }$\left({ }^{\circ} \mathrm{C}\right)$ & 1.0 & 2.0 & 3.0 & 4.0 & 5.0 & 6.0 \\
\hline 200 & $3.7936 \mathrm{E}-13$ & - & - & - & - & - \\
300 & $1.2663 \mathrm{E}-12$ & $4.1110 \mathrm{E}-12$ & $8.4941 \mathrm{E}-12$ & $1.4672 \mathrm{E}-11$ & $2.3112 \mathrm{E}-11$ & $3.4593 \mathrm{E}-11$ \\
400 & $2.8452 \mathrm{E}-12$ & $8.9139 \mathrm{E}-12$ & $1.7671 \mathrm{E}-11$ & $2.9073 \mathrm{E}-11$ & $4.3225 \mathrm{E}-11$ & $6.0323 \mathrm{E}-11$ \\
500 & $4.9729 \mathrm{E}-12$ & $1.5342 \mathrm{E}-11$ & $2.9917 \mathrm{E}-11$ & $4.8361 \mathrm{E}-11$ & $7.0554 \mathrm{E}-11$ & $9.6483 \mathrm{E}-11$ \\
600 & $7.3837 \mathrm{E}-12$ & $2.2598 \mathrm{E}-11$ & $4.3701 \mathrm{E}-11$ & $7.0038 \mathrm{E}-11$ & $1.0127 \mathrm{E}-10$ & $1.3722 \mathrm{E}-10$ \\
700 & $9.8189 \mathrm{E}-12$ & $2.9912 \mathrm{E}-11$ & $5.7574 \mathrm{E}-11$ & $9.1829 \mathrm{E}-11$ & $1.3213 \mathrm{E}-10$ & $1.7814 \mathrm{E}-10$ \\
800 & $1.2088 \mathrm{E}-11$ & $3.6719 \mathrm{E}-11$ & $7.0472 \mathrm{E}-11$ & $1.1207 \mathrm{E}-10$ & $1.6079 \mathrm{E}-10$ & $2.1613 \mathrm{E}-10$ \\
900 & $1.4076 \mathrm{E}-11$ & $4.2681 \mathrm{E}-11$ & $8.1763 \mathrm{E}-11$ & $1.2979 \mathrm{E}-10$ & $1.8585 \mathrm{E}-10$ & $2.4935 \mathrm{E}-10$
\end{tabular}


Table E-7. $\mathrm{Cu}_{2} \mathrm{O}$ solubility equation based on ref. 37 .

$$
\begin{aligned}
x & =\rho^{M} \exp (A+B / T) / P \\
M & =m_{1}+m_{3} / T
\end{aligned}
$$

$$
\begin{array}{llll}
A= & -2.14219 E+01 & m_{1}= & 6.27227 E+00 \\
B= & -4.85247 E+03 & m_{3}= & -1.30000 E-03
\end{array}
$$

Solubility (mole fraction)

\begin{tabular}{c|cccccc} 
Temperature & \multicolumn{7}{|c}{ Pressure $(\mathrm{MPa})$} \\
\cline { 2 - 7 }$\left({ }^{\circ} \mathrm{C}\right)$ & 1.0 & 2.0 & 3.0 & 4.0 & 5.0 & 6.0 \\
\hline 200 & $4.6797 \mathrm{E}-18$ & - & - & - & - & - \\
300 & $6.8329 \mathrm{E}-18$ & $3.1360 \mathrm{E}-16$ & $3.2147 \mathrm{E}-15$ & $1.8093 \mathrm{E}-14$ & $7.4398 \mathrm{E}-14$ & $2.5516 \mathrm{E}-13$ \\
400 & $8.1382 \mathrm{E}-18$ & $3.4276 \mathrm{E}-16$ & $3.1793 \mathrm{E}-15$ & $1.5910 \mathrm{E}-14$ & $5.6887 \mathrm{E}-14$ & $1.6475 \mathrm{E}-13$ \\
500 & $8.3762 \mathrm{E}-18$ & $3.3991 \mathrm{E}-16$ & $3.0298 \mathrm{E}-15$ & $1.4529 \mathrm{E}-14$ & $4.9630 \mathrm{E}-14$ & $1.3685 \mathrm{E}-13$ \\
600 & $7.8662 \mathrm{E}-18$ & $3.1309 \mathrm{E}-16$ & $2.7353 \mathrm{E}-15$ & $1.2847 \mathrm{E}-14$ & $4.2949 \mathrm{E}-14$ & $1.1582 \mathrm{E}-13$ \\
700 & $6.9768 \mathrm{E}-18$ & $2.7460 \mathrm{E}-16$ & $2.3719 \mathrm{E}-15$ & $1.1011 \mathrm{E}-14$ & $3.6379 \mathrm{E}-14$ & $9.6927 \mathrm{E}-14$ \\
800 & $5.9709 \mathrm{E}-18$ & $2.3340 \mathrm{E}-16$ & $2.0020 \mathrm{E}-15$ & $9.2289 \mathrm{E}-15$ & $3.0274 \mathrm{E}-14$ & $8.0083 \mathrm{E}-14$ \\
900 & $4.9983 \mathrm{E}-18$ & $1.9451 \mathrm{E}-16$ & $1.6610 \mathrm{E}-15$ & $7.6224 \mathrm{E}-15$ & $2.4891 \mathrm{E}-14$ & $6.5544 \mathrm{E}-14$
\end{tabular}






\section{NIST Technical Publications}

\section{Periodical}

Journal of Research of the National Institute of Standards and Technology-Reports NIST research and development in those disciplines of the physical and engineering sciences in which the Institute is active. These include physics, chemistry, engineering, mathematics, and computer sciences. Papers cover a broad range of subjects, with major emphasis on measurement methodology and the basic technology underlying standardization. Also included from time to time are survey articles on topics closely related to the Institute's technical and scientific programs. Issued six times a year.

\section{Nonperiodicals}

Monographs-Major contributions to the technical literature on various subjects related to the Institute's scientific and technical activities.

Handbooks-Recommended codes of engineering and industrial practice (including safety codes) developed in cooperation with interested industries, professional organizations, and regulatory bodies.

Special Publications-Include proceedings of conferences sponsored by NIST, NIST annual reports, and other special publications appropriate to this grouping such as wall charts, pocket cards, and bibliographies.

Applied Mathematics Series-Mathematical tables, manuals, and studies of special interest to physicists, engineers, chemists, biologists, mathematicians, computer programmers, and others engaged in scientific and technical work.

National Standard Reference Data Series-Provides quantitative data on the physical and chemical properties of materials, compiled from the world's literature and critically evaluated. Developed under a worldwide program coordinated by NIST under the authority of the National Standard Data Act (Public Law 90-396). NOTE: The Journal of Physical and Chemical Reference Data (JPCRD) is published bimonthly for NIST by the American Chemical Society (ACS) and the American Institute of Physics (AIP) Subscriptions, reprints, and supplements are available from ACS, 1155 Sixteenth St., NW, Washington, DC 20056.

Building Science Series-Disseminates technical information developed at the Institute on building materials, components, systems, and whole structures. The series presents research results, test methods, and performance criteria related to the structural and environmental functions and the durability and safety characteristics of building elements and systems.

Technical Notes-Studies or reports which are complete in themselves but restrictive in their treatment of a subject. Analogous to monographs but not so comprehensive in scope or definitive in treatment of the subject area. Often serve as a vehicle for final reports of work performed at NIST under the sponsorship of other government agencies.

Voluntary Product Standards-Developed under procedures published by the Department of Commerce in Part 10, Title 15, of the Code of Federal Regulations. The standards establish nationally recognized requirements for products, and provide all concerned interests with a basis for common understanding of the characteristics of the products. NIST administers this program in support of the efforts of privatesector standardizing organizations.

Consumer Information Series-Practical information, based on NIST research and experience, covering areas of interest to the consumer. Easily understandable language and illustrations provide useful background knowledge for shopping in today's technological marketplace.

Order the above NIST publications from: Superintendent of Documents, Government Printing Office, Washington, DC 20402.

Order the following NIST publications-FIPS and NISTIRs-from the National Technical Information Service, Springfield, VA 22161.

Federal Information Processing Standards Publications (FIPS PUB)-Publications in this series collectively constitute the Federal Information Processing Standards Register. The Register serves as the official source of information in the Federal Government regarding standards issued by N1ST pursuant to the Federal Property and Administrative Services Act of 1949 as amended, Public Law 89-306 (79 Stat. 1127), and as implemented by Executive Order 11717 (38 FR 12315, dated May 11, 1973) and Part 6 of Title 15 CFR (Code of Federal Regulations).

NIST Interagency Reports (NISTIR)-A special series of interim or final reports on work performed by NIST for outside sponsors (both government and non-government). In general, initial distribution is handled by the sponsor; public distribution is by the National Technical Information Service. Springtield. VA 22161, in paper copy or microfiche form. 
U.S. Department of Commerce

National Institute of Standards and Technology

325 Broadway

Boulder, Colorado 80303-3328

\section{Official Business}

Penalty for Private Use, $\$ 300$ 